\title{
Mechanische Spektroskopie an PMMA-Systemen: Nichtlineares Verhalten und UV-aktive Blockcopolymere
}

\section{Dissertation}

zur Erlangung des mathematisch-naturwissenschaftlichen Doktorgrades „Doktor rerum naturalium“ der Georg-August-Universität Göttingen

im Promotionsprogramm ProPhys der Georg-August University School of Science (GAUSS)

vorgelegt von

Stefanie Finkhäuser

aus Kassel

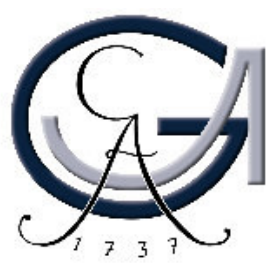

Göttingen, 2016 

Betreuungsausschuss

Prof. Dr. Konrad Samwer

Prof. Dr. Hans-Ulrich Krebs
1. Physikalisches Institut,

Georg-August-Universität Göttingen

Institut für Materialphysik,

Georg-August-Universität Göttingen

Mitglieder der Prüfungskommission

Referent:

Prof. Dr. Konrad Samwer

1. Physikalisches Institut, Georg-August-Universität Göttingen

Korreferent:

Prof. Dr. Hans-Ulrich Krebs Institut für Materialphysik,

Georg-August-Universität Göttingen

Weitere Mitglieder der Prüfungskommission:

Prof. Dr. Philipp Vana

Prof. Dr. Stefan Mathias

Prof. Dr. Wolfram Kollatschny

Dr. Richard Vink

Tag der mündlichen Prüfung:
Institut für Physikalische Chemie,

Georg-August-Universität Göttingen

1. Physikalisches Institut,

Georg-August-Universität Göttingen

Institut für Astrophysik

Georg-August-Universität Göttingen

Institut für Materialphysik,

Georg-August-Universität Göttingen 

Meiner Familie 



\section{Inhaltsverzeichnis}

$\begin{array}{lr}\text { 1. Einleitung } & 9\end{array}$

2. Theoretische Grundlagen $\quad 11$

2.1. Gläser und der Glasübergang . . . . . . . . . . . . . . . . . . . . 11

2.1.1. Der Glasbegriff . . . . . . . . . . . . . . . . . . . . . . . . . . . . . 11

2.1.2. Glasübergang in Polymersystemen . . . . . . . . . . . . 12

2.1.3. Relaxationen im Glaszustand . . . . . . . . . . . . . 13

2.1.4. Relaxationszeiten ............... . . . 15

2.1.5. Potentielle Energielandschaft . . . . . . . . . . . . 17

2.2. Mechanische Eigenschaften von Gläsern . . . . . . . . . . . . . . . . . . . . . . . . . . . . . 19

2.2.1. Viskoelastizität . . . . . . . . . . . . . . 19

2.2.2. Dynamisch mechanische Spektroskopie an viskoelastischen Ma-

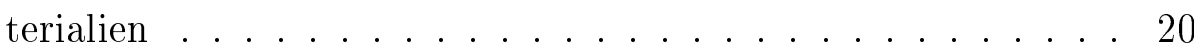

2.3. Charakteristische Eigenschaften von Polymeren . . . . . . . . 22

2.3.1. Struktur der Polymere . . . . . . . . . . . . . . . 22

2.3.2. Polymerdynamik . . . . . . . . . . . . . 23

2.3.3. Blockcopolymere und Mikrophasenseparation ....... . 25

2.4. Nichtlineares Verhalten amorpher Materie . . . . . . . . . . . . 27

2.4.1. Nichtlineare Antwort . . . . . . . . . . . . . . . 27

2.4.2. Box Modell und dielektrische Spektroskopie . . . . . . . . 31

2.4.3. Kooperativität und höhere nichtlineare Suszeptibilitäten . . 32

3. Experimentelle Methoden $\quad 35$

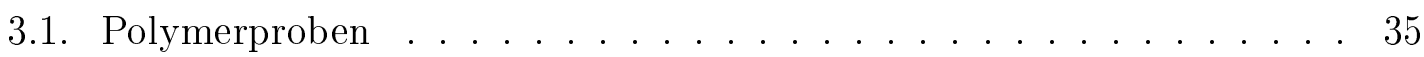

3.2. Thermogravimetrische Analyse . . . . . . . . . . . . . . . . . 36

3.3. Dynamisch mechanische Messungen . . . . . . . . . . . . . 38

3.3.1. Aufbau und Spezifikation . . . . . . . . . . . . . 38

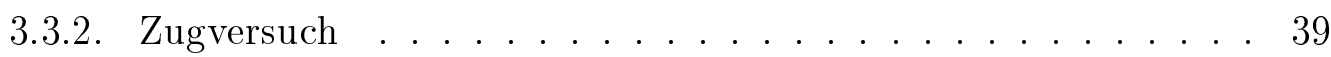

3.3.3. Biegebalkenexperiment . . . . . . . . . . . 40

3.3.4. Temperaturscans der Blockcopolymere . . . . . . . . . . 40 40

3.3.5. Versuche mit UV-Bestrahlung . . . . . . . . . . . . . . . . 42

3.3.6. LAOS-Messtechnik und Modifikation DMA8000 . . . . . . 42

3.4. Kleinwinkel-Röntgenstreuung . . . . . . . . . . . . . . 45

3.5. Rasterkraftmikroskopie . . . . . . . . . . . . . 45 
4. Nichtlineares Verhalten von PMMA 49

4.1. Grundelegende mechanische Eigenschaften . . . . . . . . . . . 49

4.2. Nichtlinearität in dynamisch mechanischen Messungen . . . . . . . 51

4.3. Zeitabhängigkeit der nichtlinearen Effekte . . . . . . . . . . 57

4.3.1. Nichtlineares zeitabhängiges Verhalten von $\operatorname{PMMA}^{E} \ldots \ldots 58$

4.3.2. Nichtlineares zeitabhängiges Verhaltens für PMMA ${ }^{G} \ldots .63$

4.3.3. Quantifizierung der nichtlinearen Effekte über die Nachgibiegkeiten höherer Ordnung $\ldots \ldots \ldots \ldots 7 . \ldots . \ldots 67$

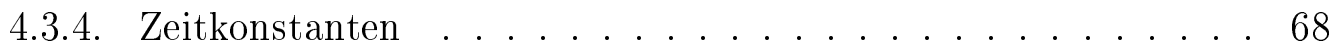

4.3.5. Zusammenfassung der Ergebnisse … . . . . . . 70

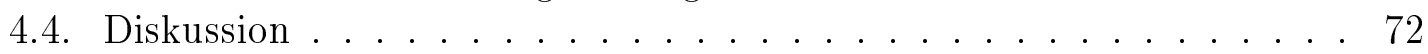

4.4.1. Detektion des nichtlinearen Verhaltens an PMMA $^{E} \ldots \ldots 72$

4.4.2. Zeitabhängige Messungen … . . . . . . . . 74

4.4.3. Temperatur- und Spannungsabhängigkeit der Plateauwerte für

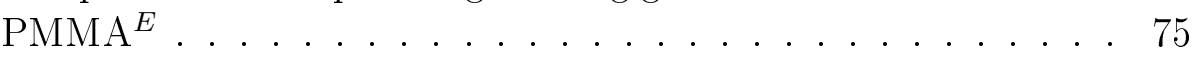

4.4.4. Temperatur- und Spannungsabhängigkeit der Plateauwerte für

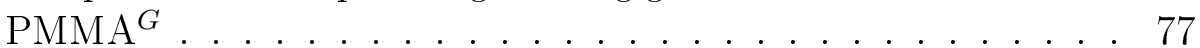

4.4.5. Vergleich der Messungen an $\mathrm{PMMA}^{E}$ und $\mathrm{PMMA}^{G} \ldots \ldots 77$

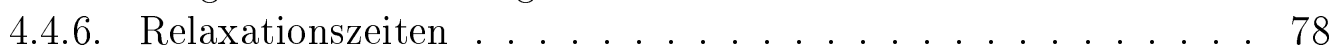

5. UV-aktive Blockcopolymere 81

5.1. Thermische Stabilität . . . . . . . . . . . . . . . 81

5.2. Temperaturabhängigkeit der mechanischen Eigenschaften . . . . . 83

5.2.1. Einzelne Komponenten der BCPs . . . . . . . . . . . 83

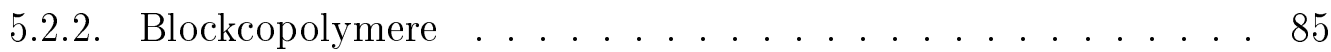

5.3. Kleinwinkelröntgenstreuung . . . . . . . . . . . . . 9 90

5.4. Lichtinduziertes Crosslinking und Decrosslinking . . . . . . . . . 92

5.5. Ordnungs-Unordnungs-Übergang $\ldots \ldots \ldots \ldots \ldots$

5.6. Zusammenfassung der Ergebnisse $\ldots \ldots \ldots$. . . . . . . 100

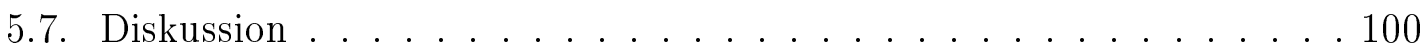

5.7.1. Morphologie . . . . . . . . . . . . . . . . . . . 101

5.7 .2 . Vernetzung und Entnetzung mit UV-Licht $\ldots . . . . .106$

5.7.3. Ordnungs-Unordnungsübergang $\ldots \ldots \ldots \ldots$

$\begin{array}{ll}\text { 6. Zusammenfassung und Ausblick } & 111\end{array}$

$\begin{array}{ll}\text { Literatur } & 120\end{array}$

$\begin{array}{ll}\text { Abbildungen } & 126\end{array}$

$\begin{array}{ll}\text { A. Anhang } & 127\end{array}$

$\begin{array}{ll}\text { B. Abkürzungen } & 133\end{array}$ 


\section{Einleitung}

Polymere sind große Moleküle, die aus einzelnen Einheiten, den Monomeren zusammengesetzt sind. Polymerische Materialien werden in allen Bereichen des täglichen Lebenes angetroffen und werden häufig aufgrund ihrer besonderen mechanischen Eigenschaften geschätzt. Schon vor dem Beginn der Kunststoffindustrie, die durch die Vulkanisierung des Gummi (1839) ihren Anfang nahm, war beispielsweise Seide aufgrund ihrer starken Festigkeit und einem gleichzeitigen geringen Gewicht sehr geschätzt. Nachdem Staudinger Anfang der 1920er Jahre mit seiner Arbeit den Grundstein für die moderne Makromolekulare Chemie gelegt hatte ([88], [87]) folgte eine rasante Entwicklung neuer synthetischer Poylmerwerkstoffe. Insbesondere das zunehmende Verständnis der Verknüpfung der Struktur der Polymere mit ihren physikalischen Eigenschaften, hat dazu geführt, dass eine Vielzahl neuer Kunststoffe mit speziellen auf ihre Anwendung zugeschnittenen mechanischen Eigenschaften entwickelt wurden. Beispiele dafür sind moderne Autoreifen, funktionelle Textilfasern und Anwendungen in der Lebensmittel- oder Kosmetikindustrie.

Ein physikalisches Phänomen, welches unter anderem für die mechanischen Eigenschaften einer Vielzahl polymerer Werkstoffe grundlegend ist, ist der Glasübergang. Große Wichtigkeit besitzt er für die Produktion, da viele Kunststoffe im Glaszustand geformt werden können und er bestimmt das mechanische Verhalten grundlegend. Der Glasübergang und seine Physik sind bis heute nicht vollständig verstanden und daher Gegenstand aktueller Forschung in der Festkörperphysik. Insbesondere das Verhalten von Gläsern unter dem Einfluss großer externer Felder ist bisher kaum erforscht. Während der Verarbeitung vieler amorpher Polymerwerkstoffe, wie beispielsweise dem Spritzgussverfahren, wird das Material jedoch häufig sehr großen Spannungen ausgesetzt, so dass das Verhalten des Materials sich nicht mehr mit den wohlbekannten einfachen linearen Zusammenhängen beschreiben läßt. Amorphe Materialien verhalten sich bei niedrigen Temperaturen und kleinen Spannungen quasi elastisch, zeigen also global eine lineare Abhängigkeit von Dehnung und Spannung. Werden Temperatur oder Spannung ausreichend groß, so wird nichtlineares Verhalten beobachtet. Um diesen Übergang vom linearen zum nichtlinearen Verhalten zu charakterisieren, wird mechanische Spektroskopie mit großen Amplituden verwendet. Die auftretende Nichtlinearität ist eng mit der Struktur des amorphen Materials verknüpft und kann daher zu weiteren Informationen über die Natur und den mikroskopischen Hintergrund des Glasüberganges führen.

Der Glasübergang ist das bestimmende physikalische Phänomen in amorphen Poylmeren. Jedoch gibt es viele Polymersysteme, die in einem ganz oder teilweise geord- 


\section{Einleitung}

neten Zustand vorliegen. Insbesondere die Blockcopolymere, also Polymere die aus zwei Blöcken unterschiedlicher Monomere bestehen, haben in letzter Zeit viel Interesse auf sich gezogen. Aufgrund der Mikrophasenseparation die zur Ausbildung von geordneten Strukturen im Nanometerberich führt, sind sie besonders interessant für Anwendungen. Ein Überblick über die jüngsten Anwendungen ist in [80] zu finden.

Besonders interessant sind hierbei UV-aktive Blockcopolymersysteme, da es möglich ist durch eine Bestrahlung mit UV-Licht die Polymere zu vernetzen. Dadurch können Nichtgleichgewichtszustände eingefroren werden und somit im Detail untersucht werden. Auch Übergänge zwischen zwei Nichtgleichgewichtszuständen sind somit experimentell zugänglich. Die mechanischen Eigeschaften von einem solchen Sytem sind von großem Interesse, da die mikrophasenseparierte Struktur für eine heterogene Verteilung der lokalen mechanischen Eigenschaften sorgt.

Die vorliegende Arbeit beschäftigt sich mit zwei verschiedenen Schwerpunkten. Einerseits wurde im Rahmen der vorliegenden Arbeit das Auftreten höherer nichtlinearer Beiträge mittels mechanischer Spektroskopie für Polymethylmethacrylat (PMMA) untersucht. Dafür wurde in Anlehnung an dielektrische Messungen bei großen Feldern, ein Experiment entwickelt, das diese Messmethode für das mechanische Verhalten überträgt. Die Ergebnisse dieser mechanischen Messungen werden in dieser Arbeit auf Konsistenz überprüft. Der zweite Teil der Arbeit behandelt die Mikrophasenseparation von UV-aktiven Blockcopolymeren. Dafür wurden Blockcopolymere verschiedener Zusammensetzung mithilfe dynamisch mechanischer Messungen hinsichtlich ihrer temperaturabhängigen mechanischen Eigenschaften untersucht. Insbesondere wurde auch untersucht, ob eine lichtinduzierte Vernetzung sowie eine Entnetzung der Blockcopolymere möglich ist. Zunächst werden im Kapitel $\underline{2}$ die theoretischen Grundlagen bereitgestellt. Im anschließenden Kapitel $\underline{3}$ werden die verwendeten experimentellen Techniken behandelt. Nachfolgend werdēn die Ergebnisse der Messungen zum nichtlinearen mechanischen Verhalten von PMMA im Kapitel $\underline{4}$ dargestellt und diskutiert. Das Kapitel 5 zeigt die Ergebnisse der Untersuchungen an den UV-aktiven Blockcopolymeren und deren Diskussion. Abschließend wird in Kapitel $\underline{6}$ eine Zusammenfassung der Arbeit und ein Ausblick gegeben. 


\section{Theoretische Grundlagen}

Dieser Abschnitt der Arbeit behandelt die theoretischen Grundlagen und Zusammenhänge, die zum Verständnis der folgenden Kapitel benötigt werden.

\subsection{Gläser und der Glasübergang}

\subsubsection{Der Glasbegriff}

Als Glas werden in der Physik amorphe Feststoffe bezeichnet, die einen Glasübergang zeigen [28]. Amorphe Materialien zeigen im Gegensatz zu Kristallen keine langreichweitige Ordnung ihrer mikroskopischen Bausteine. Diese mikroskopischen Bausteine können, wie im Fall der metallischen Gläser, einzelne Atome sein, oder auch relativ große Moleküle wie in einem Polymer. Somit gibt es eine Vielzahl unterschiedlicher Materialien in der Klasse der Gläser, was zu einer großen Bandbreite physikalischer Phänomene auf diesem Gebiet führt. Besonders interessant ist dabei jedoch, dass diese Materialien trotzdem ein universelles Verhalten, den Glasübergang zeigen. Dieser ist bis heute noch nicht vollständig verstanden.
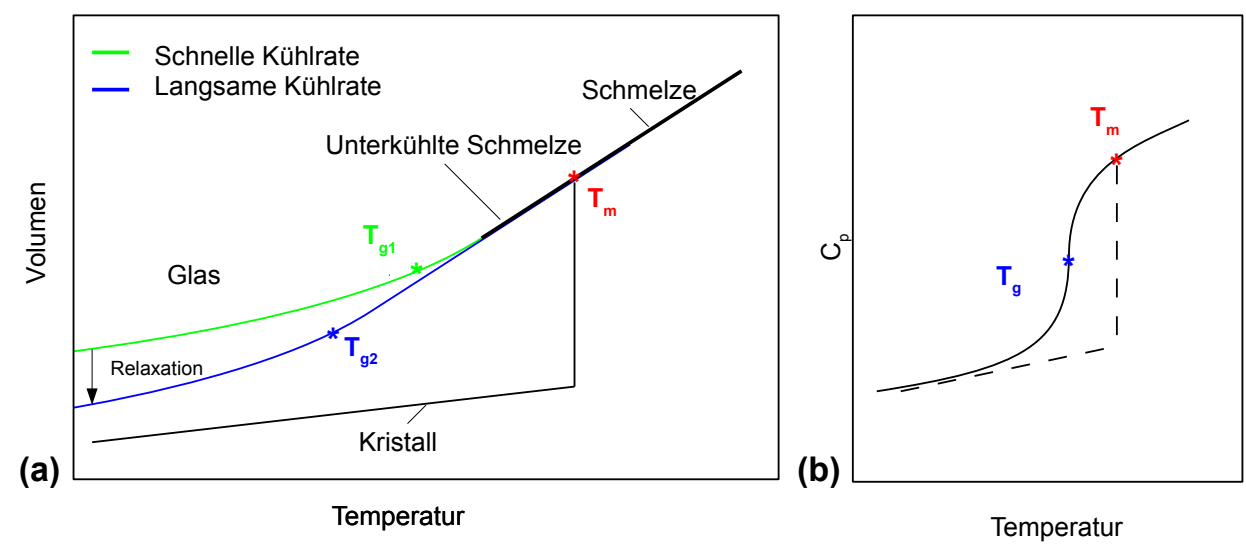

Abb. 2.1.: Schematisch dargestellte Temperaturabhängigkeit (a) des Volumens eines Glasbildners, (b) der spezifischen Wärme eines Glases. Nach [28]

Wird ein glasbildendes Material so schnell abgekühlt, dass Kristallisation vermieden wird, so zeigt sich mit sinkender Temperatur ein Abfall des Volumens, dessen Steigung sich an einem gewissen Punkt, dem Glasübergang ändert. Dies ist in der 


\section{Theoretische Grundlagen}

Abbildung 2.1(a) dargestellt. Im Gegensatz zur Klassifikation der Kristallisation, welche eindeutig ein Phasenübergang 1.Ordnung ist, kann der Glasüberganges nicht eindeutig im Ehrenfestschen Schema eingeordnet werden, obwohl er einige Züge eines Phasenüberganges 2.Ordnung zeigt. Sollte es sich nämlich beim Glasübergang um einen Phasenübergang 2.Ordnung handeln, so erwartet man nach Ehrenfest [26] einerseits eine Unstetigkeit in der 1.Ableitung der Gibbschen Enthalpie, also z.B. im Volumen oder der Entropie und zudem einen Sprung in einer 2.Ableitung der Gibbschen Enthalpie, beispielsweise in der spezifischen Wärme $c_{p}$, die experimentell gut zugänglich ist. Die Abbildung 2.1(b) zeigt den schematischen Verlauf von $c_{p}$ für ein Glas. Es wird jedoch statt eines Sprunges eine s-förmige Stufe beobachtet. Die Höhe der Stufe in $c_{p}$ gibt Aufschluss, ob es sich um einen starken Glasbildner oder einen fragilen Glasbildner handelt. Die Einteilung in starke und fragile Gläser wird im Abschnit 2.1.3 genauer thematisiert. Ein weiterer Unterschied zu einem klassischen Phasenübergang ist, dass der Glasübergang kühlratenabhängig ist. Je langsamer das Material gekühlt wird, desto geringer ist die beobachtete Glastemperatur. Es herrscht folglich auch kein Gleichgewichtszustand bei $T_{g}$, was eine Voraussetzung für eine echte thermodynamische Umwandlung ist. Häufig wird von einem metastabilen Gleichgewicht gesprochen. Da der Glasübergang kein echter thermodynamischer Phasenübergang ist, ist sein experimentell bestimmter Wert abhängig von den äußeren Parametern der Messung, sowie von der gewählten Messmethode. Damit sind Glasübergangstemperaturen nur dann direkt reproduzierbar, wenn das Material eine identische Vorgeschichte durchlaufen hat, und mit identischen Messparametern mit der gleichen Methode gemessen wird. Um diese Vergleichbarkeit zu gewährleisten, wurden in dieser Arbeit stets das gleiche Temperaturprotokoll und dieselben Messparameter verwendet.

\subsubsection{Glasübergang in Polymersystemen}

Viele Polymere sind sehr gute Glasbildner und zeigen häufig auch gar keine Kristallisation. Dies liegt an der komplexen und häufig stark unsymmetrischen Molekülstruktur, sowie der hohen Viskosiät in der flüssigen Phase. Die Höhe der Glasübergangstemperatur ist bei Polymeren durch verschiedene Faktoren beeinflusst. Betreffend die chemischen Struktur, hat die Flexibilität der Hauptkette, die durch die Konstituenten festgelegt ist, den größten Einfluss. Dabei ist es von Bedeutung, ob die Strukturelemente um die Achse der Hauptkette verdrehbar sind. Bei einer festen Temperatur haben die Polymere mit höheren Glastemperaturen zudem typischerweise auch einen höheren Modul. Beispielsweise ist Polyethylen sehr flexibel und zeigt daher ein niedriges $T_{g}$ von $-133^{\circ} \mathrm{C}$. Im Gegensatz dazu weist Polymethylmethacrylat eine relativ hohe Glasübergangstemperatur von ca. $105^{\circ} \mathrm{C}$ auf. Desweiteren führen große Seitengruppen zu einer Festigung der Hauptkette. Insbesondere große polare Seitengruppen führen zu einem erhöhten $T_{g}$ [92]. Verzweigung und Vernetzung führen zu einer reduzierten Beweglichkeit der Hauptkette und führen daher häufig auch zu einer höheren Glastemperatur [62]. 
Einen weiteren wichtigen Einfluss auf die Höhe des Glasüberganges hat die Molmasse $M_{W}$ der Polymere. Für größere Molmassen werden höhere Werte von $T_{g}$ beobachet und es gilt der folgende empirische Zusammenhang, die sogenannte Flory-FoxGleichung [31]:

$$
T_{g}=T_{g, \infty}-K / M_{W}
$$

Hierbei ist $T_{g, \infty}$ die theoretische Glastemperatur für das Polymer mit unendlicher Molmasse und $K$ eine Konstante $>0$. Das bedeutet, dass für kleine Molmassen $\left(<10^{2}\right)$ die beobachtete Glastemperatur linear mit $M_{W}$ ansteigt. Für größere Molmassen wird die Abhängigkeit geringer und sättigt schließlich bei sehr hohen Molmassen $\left(\approx 10^{4} \mathrm{~g} / \mathrm{mol}-10^{5} \mathrm{~g} / \mathrm{mol}\right)$.

\subsubsection{Relaxationen im Glaszustand}

Dynamische Prozesse spielen eine große Rolle bei der Beschreibung von Gläsern, denn der Glasübergang kann als ein Einfrieren der Molekulardynamik im Material verstanden werden. Im Hinblick auf diese Dynamik wird die experimentelle Beobachtungszeit sehr wichtig. Die Zeiten $\tau$, die für molekulare Bewegungen und Umorientierungen im Glas nötig sind erstrecken sich in der Nähe der Glastemperatur typischerweise in einer Größenordnung von $\tau \approx 100 \mathrm{~s}$. Besonders die Unterscheidung zwischen Flüssigkeit und Festkörper ist abhängig von der Beobachtungszeit, denn typische Gläser verhalten sich für kurze Beobachtungszeiten wie ein Festkörper und doch fließen sie und zeigen bei langen Beobachtungszeiten ein flüssikeitsartiges Verhalten. Diese Charakteristik von Gläsern gleichzeitig viskos und elastisch zu reagieren wird als Viskoelastizität bezeichnet und in Abschnitt 2.2.1 näher erläutert. Trotzdem kann die Viskosität herangezogen werden um zwischen Flüssigkeit und Glas zu unterscheiden. Es wurde festgelegt, dass ein amorphes Material dann als eine Flüssigkeit bezeichnet wird, wenn die Viskosität $\eta$ unterhalb von $10^{12}$ Pas liegt. Damit kann über die Viskosität die Glastemperatur wie folgt definiert werden :

$$
T_{g}=T\left(\eta=10^{12} \mathrm{Pas}\right)=T(\tau=100 \mathrm{~s})
$$

Die Viskosität eines Glases im flüssigen Zustand ändert sich sehr stark bei Annäherung an $T_{g}$. Sie erstreckt sich dabei über bis zu 16 Größenordnungen. Die Temperaturabhängigkeit der Viskosität für verschiedene glasbildende Materialien ist in Abbildung 2.2, dem Angell-Plot abgebildet. Dabei wurde der Logarithmus der Viskosität gegen die inverse Temperatur multipliziert mit $T_{g}$ aufgetragen. Messgrößen, denen thermisch aktivierte Sprünge über eine Energiebarriere $E$ zugrundeliegen zeigen in einer Auftragung gegen 1/T typischerweise eine Gerade, deren Steigung gegen $1 / k_{B} T$ die Höhe der Energiebarriere liefert [43]. Dieses Arrhenius-Verhalten wird jedoch nur von einem Teil der Gläser gezeigt. Sie werden als starke Gläser bezeichnet. 
2. Theoretische Grundlagen

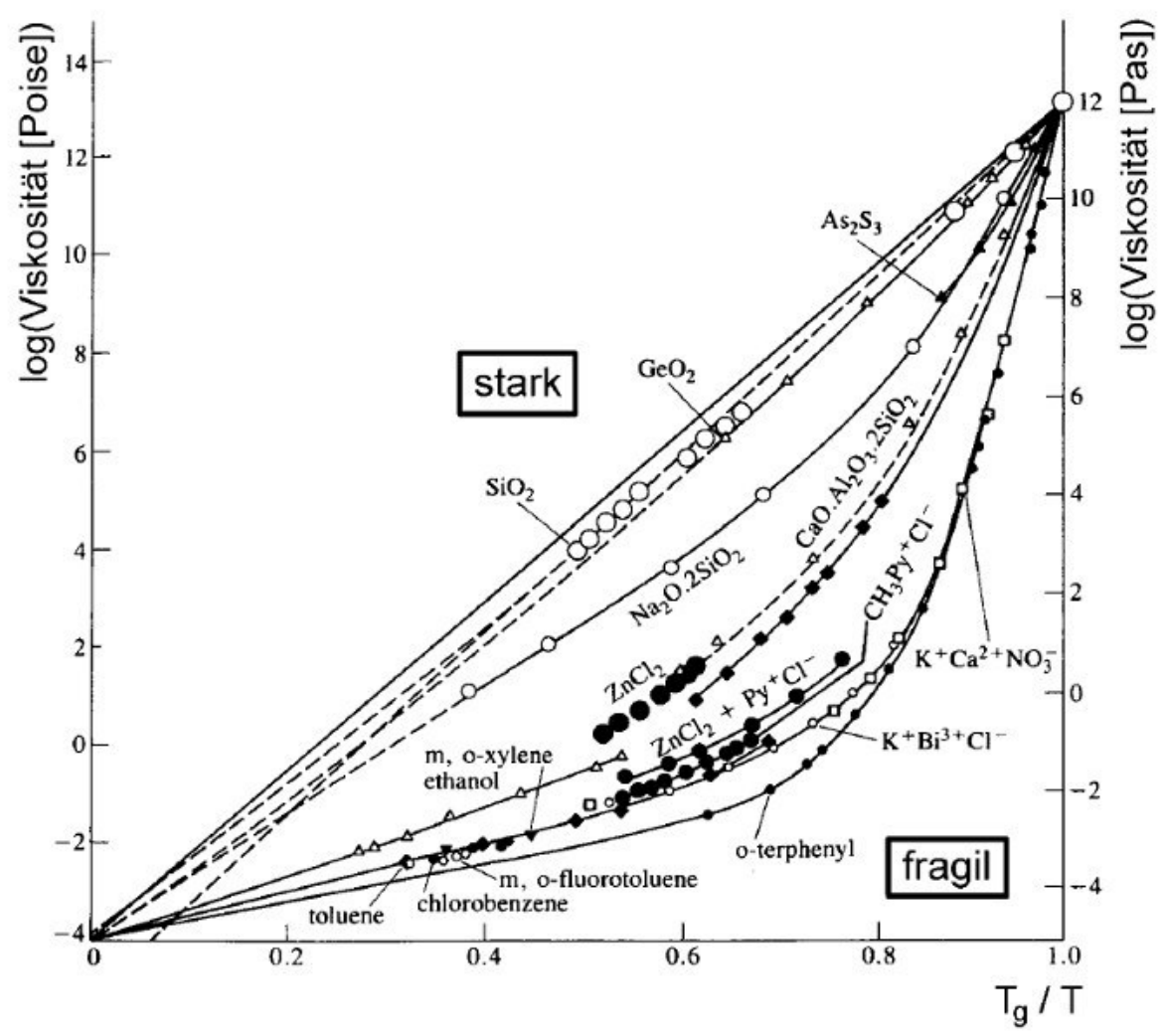

Abb. 2.2.: Angell-Plot: Temperaturabhängigkeit der Viskosität oberhalb der Glastemperatur für verschiedene Gläser [1]. 


$$
\eta=\eta_{0} \cdot \exp \left(\frac{E_{A}}{k_{B} T}\right)
$$

Die andere Klasse von Gläsern zeigt einen gekrümmten Verlauf im Angell-Plot. Sie werden als fragil bezeichnet und ihre temperaturabhängige Viskosität läßt sich mittels des Vogel-Fulcher-Tammann-Gesetzes beschreiben [96], [32], [90]:

$$
\eta=\eta_{0} \cdot \exp \left(\frac{D \cdot T_{V F T}}{T-T_{V F T}}\right)
$$

Um zwischen starken und fragilen Gläsern zu unterscheiden, wird ein Fragilitätsindex $m$ definiert.

$$
m=\left.\frac{d \log \eta}{d\left(T_{g} / T\right)}\right|_{T_{g}=T}
$$

Die meisten Polymere zeigen ein fragiles Verhalten. Für hohe Temperaturen geht das Vogel-Fulcher-Tammann Gesetz in ein Arrhenius-Verhalten [2] mit der Energiebarriere D über, während es mit sinkender Temperatur eine Zunahme der effektiven Energiebarriere zeigt.

\subsubsection{Relaxationszeiten}

Zunächst soll anhand eines einzelnen Relaxators (Debye-Relaxator, [21]) das Verhalten eines rein thermisch aktivierten Prozesses erläutert werden. Zugrunde liege ein Doppelmuldenpotential mit der Energiebarriere E. Die Wahrscheinlichkeit eines Teilchens von einer der Mulden in die andere zu gelangen hängt von der äußeren Temperatur ab. Ist die Energie im System kleiner als $E_{A}$, so kann das Teilchen lediglich eine harmonische Schwingung der Frequenz $f_{0}$ in einer der Mulden ausführen. Die Häufigkeit der Sprünge über die Barriere kann über eine Sprungfrequenz $f_{s}$ beschrieben werden.

$$
f_{s}=f_{0} \cdot \exp \left(-E_{A} / k_{B} T\right)
$$

Liegt eine der Mulden tiefer, so ist es wahrscheinlicher das Teilchen nach einer Zeit $t$ dort zu finden. Für eine Messgröße $\phi$, die von der Aufenthaltswahrscheinlichkeit des Teilchens abhängt, ergibt sich dann ein exponentieller Abfall mit einer Relaxationszeit $\tau$.

$$
\phi(t)=\phi_{0} \exp \left(f_{s} t\right)=\phi_{0} \cdot \exp (-t / \tau)
$$

Im Experiment ist dieses Verhalten jedoch für den Glasübergang nicht typisch. Ein Prozess mit einer einzelnen Relaxationszeit ist der $\beta$-Prozess, der bei Temperaturen unterhalb von $T_{g}$ auftritt und häufig im Experiment als eine Flanke des $\alpha$-Peaks beobachtet wird. Stattdessen folgen die Daten für einen Glasübergang häufig dem 


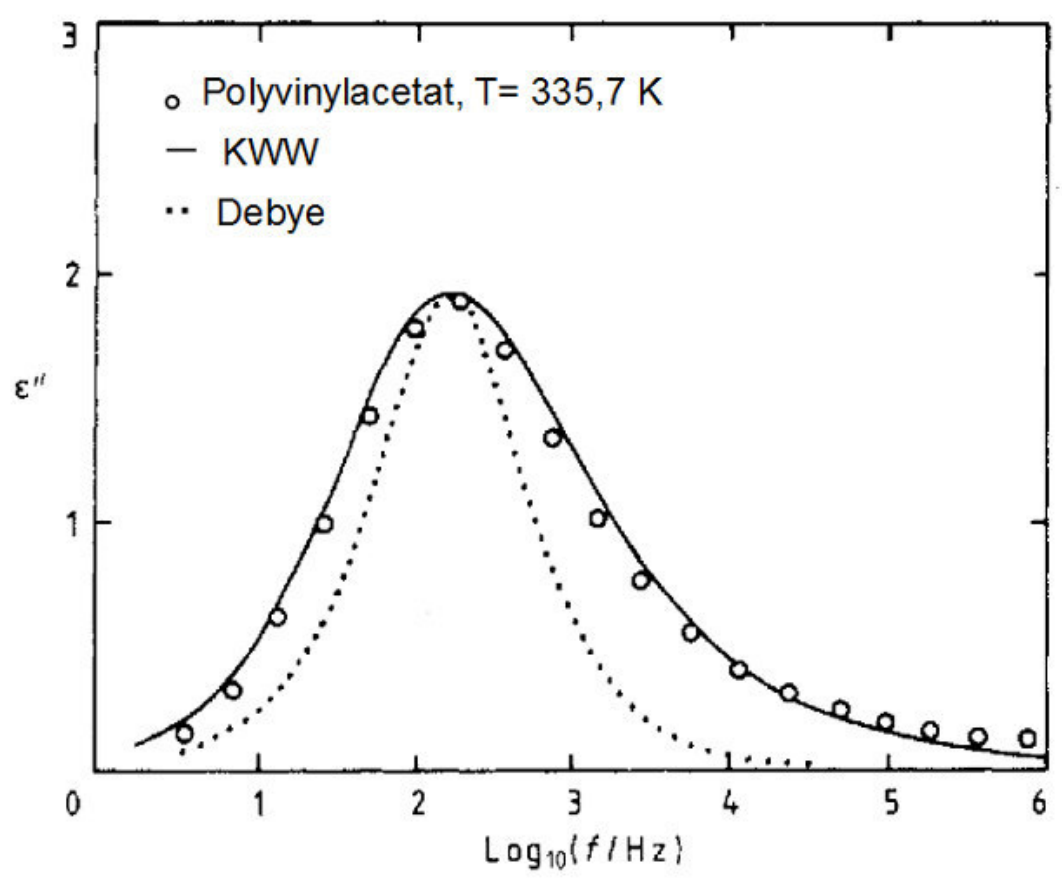

Abb. 2.3.: Frequenzabhängigkeit des dielektrischen Verlustes $\epsilon$ " aus [54]. Die Messung stammt von Ishida et al. [53].

empirischen Kohlrausch-William-Watts-Gesetz [58], [99]:

$$
\phi(t)=\phi_{0} \exp \left(-(t / \tau)^{\beta}\right)
$$

Es handelt sich hierbei um eine gestreckte Exponentialfunktion (Streckfaktor $\beta<1$ ). Im Frequenzraum zeigen die Debye- und die KWW-Funktion das in Abbildung 2.3 dargestellte Verhalten. Deutlich ist, dass die Kohlrauschfunktion eine breitere und zudem unsymmetrische Frequenzverteilung liefert. Als Erklärung für diesen in Experimenten häufig gefundenen Zusammenhang gibt es verschiedene Ansätze. Der einfachste geht von einer homogenen Verteilung aus, so dass überall im amorphen Material das gestreckte KWW-Relaxationsverhalten beobachtet würde. Es gibt jedoch viele Veröffentlichungen über das Auftreten von dynamischen Heterogenitäten in amorphen Materialien, die im Gegensatz zu dieser einfachen Vorstellung sind. Eine Übersicht dieser Veröffentlichungen bietet das Review [84]. Grundlage der dynamischen Hereogenitäten ist die Annahme, dass es im Material eine statistische Verteilung von schnell und langsam relaxierenden Bereichen gibt, die zu dem beobachteten Kohlrauschverhalten führen. Es handelt sich also um ein kooperatives Phänomen, bei dem es verschiedene Beiträge zu den Relaxationszeiten gibt. Im Abschnitt 2.4 wird das Konzept der dynamischen Heterogenitäten ausführlich behandelt.

Desweiteren, kann eine Verknüpfung des KWW-Exponenten $\beta$ mit dem Fragilitäts- 


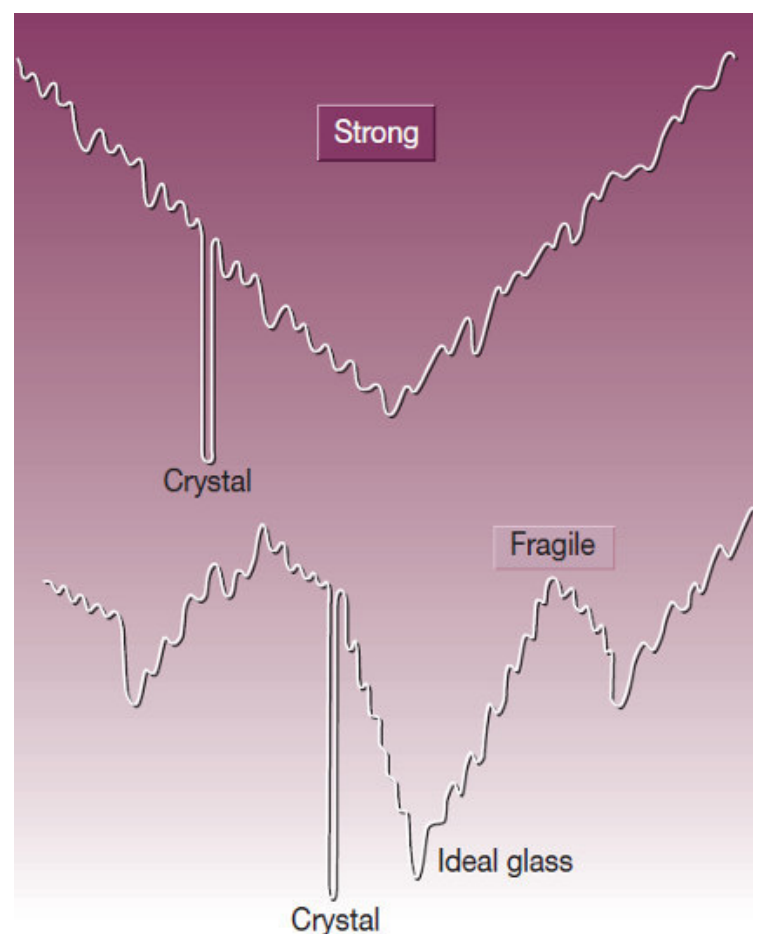

Abb. 2.4.: Schematische Darstellung der PEL für ein starkes und ein fragiles Glas (aus [20])

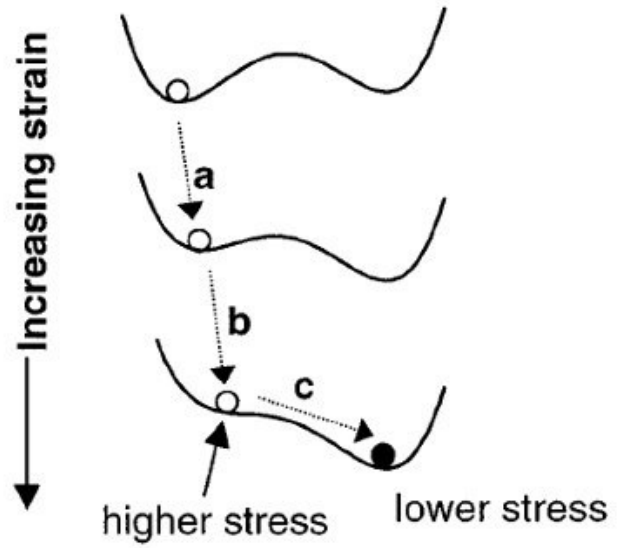

Abb. 2.5.: Schematische Darstellung des Einflusses von mechanischer Dehung auf eine Energiebarriere. [60]

index $m$ beobachtet werden: Nach Böhmer gilt der empirische Zusammenhang [4]:

$$
m \approx(250 \pm 30)-320 \cdot \beta_{K W W}
$$

Für das in dieser Arbeit untersuchte Polymethylmethacrylate wird in der Literatur [69] ein Wert von 0,34 für $\beta_{K W W}$ im Bereich des Glasüberganges gefunden.

\subsubsection{Potentielle Energielandschaft}

Im folgenden Abschnitt soll das Konzept einer potentiellen Energielandschaft (PEL) zur Beschreibung der Phänomenologie von glasbildenden Systemen nach Debendetti und Stillinger [20] vorgestellt werden. Den Grundgedanken für diese Beschreibung legte Goldstein 1969 [36], als er für die potentielle Energie von Gläsern eine Struktur vorschlug, die durch Energiebarrieren gekennzeichnet ist, welche hoch sind im Vergleich zur thermischen Energie. Die Funktion der potentiellen Energie $V\left(r 1, \ldots . . r_{N}\right)$ für ein System aus N Teilchen ist abhängig von den jeweils 3 Ortskoordinaten jedes der Teilchen. Da es im Festkörper zu vielen Wechselwirkungen der Teilchen untereinander kommt, müssen diese berücksichtigt werden. Damit ergibt sich eine N-Teilchen Funktion, so dass die Funktion der potentiellen Energie $V$ ein $3(\mathrm{~N}-1)$-dimensionales 


\section{Theoretische Grundlagen}

Objekt ist. Diese multidimensionale Energiefunktion wird als die potentielle Energielandschaft bezeichnet. Handelt es sich bei den Teilchen nicht um einzelne Atome, sondern beispielsweise um Moleküle, so kommen zusätzliche Freiheitsgrade für Orientierung und Rotation dazu. Eine schematische Darstellung der potentiellen Energielandschaft ist in Abbildung $2.4 \mathrm{zu}$ sehen. Von besonderem Interesse sind die globalen Minima, und die Barrieren zwischen ihnen. Dabei untescheidet man sogenannte Megabasins, welche durch eine besonders niedrige potentielle Energie gekennzeichnet sind und Subbasins, die eine Art Unterstruktur bilden, da zwischen ihnen die Barrieren vergleichsweise niedrig sind. Die PEL für ein N-Teilchen System bei konstantem Volumen ist fest und das unterschiedliche Verhalten des Materials wird darauf zurückgeführt, dass je nach äußeren Bedingungen wie Temperatur und Druck, verschiedene Bereiche der PEL zugänglich sind. Das dynamische Verhalten des Materials ist dabei dadurch bestimmt, wie in Abhängigkeit der Temperatur die PEL erkundet wird, während die Art, wie sich die Landschaft aufgrund von Änderungen der Dichte verformt, die mechanischen Eigenschaften festlegt [42].

Anhand von Molekular-dynamischen Simulationen konnten drei verschiedene Temperaturbereiche gefunden werden, in denen jeweils die PEL auf bestimmte Weise erkundet wird [79]. Für sehr hohe Temperaturen ist die Dynamik des Systems temperaturunabhängig, da die Barrieren im Vergleich zur thermischen Energie sehr klein sind und damit dem System die gesamte Energielandschaft zugänglich ist (freie Diffusion). Für tiefere Temperaturen gibt es einige Barrieren, welche hoch sind im Vergleich der thermischen Energie, so dass das System in einem bestimmten Bereich der PEL „gefangen“ ist. Hierbei ändert sich das Verhalten und für die strukturelle Relaxation wird das gestreckte exponentielle Verhalten beobachtet. Damit wird die Aktivierungsenergie temperaturabhängig und steigt mit sinkender Temperatur. Sobald die Temperatur so niedrig ist, dass das System in einem einzelnen Minimum gefangen ist, spricht man von einem Glas. Dabei wird die Tiefe dieses Minimums durch die Kühlrate bestimmt.

Es wird angenommen, dass für die Verteilung der jeweiligen Tiefen der Minima und die Höhe der Sattelpunkte entlang der Pfade benachbartert Basins ein Skalengessetz besteht, welches helfen könnte die Verknüpfung von Dynamik und Thermodynamik in Gläsern besser zu verstehen [20]. Wie im Abschnitt 2.1.3 beschrieben, werden für Gläser zwei verschiedene Arten von Tempraturabhängigkeit der Relaxationszeiten bzw. der Viskosität beobachtet. Diese fragilen und starken Gläser können im Rahmen der PEL dadurch unterschieden werden, dass für starke Gläser eine relativ homogene PEL zugrundeliegt, welche jedoch durch ein einzelnes tiefes Minimum (Megabasin) gekennzeichnet ist. In einem fragilen Glas hingegen liegt die PEL sehr heterogen vor. Das bedeutet, es gibt mehrere Megabasins. In der Abbildung 2.4 ist jeweils die PEL für ein starkes und ein fragiles Glas dargestellt.

Im Experiment werden durch Temperaturänderung verschiedene Bereiche der PEL für die Probe zugänglich. Somit führt eine Erhöhung der Temperatur dazu, dass die mittlere Energie der besuchten lokalen Minima ebenfalls steigt. Dadurch läßt sich 
eine fiktive Temperatur definieren, die angibt, welche Minima in der PEL für ein System im Gleichgewicht zugänglich sind. Weiteren Einfluss auf das Material hat eine mechanische Einwirkung durch äußere Spannungen. MD-Simulationen konnten zeigen, dass in einem System, welches sich in einem lokalen Minimum der PEL befindet, unter Dehnung zunächst kontinuierlich Spannung und Energie steigen, jedoch zusätzlich immer wieder sogenannte Stress drops beobachtet werden [22], [65], [95]. Es konnte gezeigt werden, dass externe Dehnung des Systems zum Verschwinden eines Energiminimums führt und damit das System in ein anderes Minimum mit einem geringere Spannungszustand zwingt. Es werden folglich dehnungs-aktivierte Relaxationen für hohe externe Spannungen erwartet [60]. Dieses Szenario ist in der Abbildung 2.5 schematisch dargestellt.

\subsection{Mechanische Eigenschaften von Gläsern}

\subsubsection{Viskoelastizität}

Gläser zeigen viskoelastisches Verhalten, das bedeutet, sie reagieren sowohl elastisch als auch viskos auf eine äußere Spannung $\sigma$. Für ein rein elastisches Verhalten gilt das Hooksche Gesetz und die durch die äußere Spannung hervorgerufene Dehnung $\epsilon$ ist proportional zur Spannung.

$$
\sigma=E \cdot \epsilon
$$

Unter der Annahme, dass die untersuchten Materialien isotrop sind, wird hier auf eine vektorielle bzw. tensorielle Schreibweise verzichtet. Die Proportionalitätskonstante in der obigen Gleichung ist der Modul, er ist materialspezifisch. Flüssigkeiten hingegen zeigen eine Proportionalität der äußeren Spannung zur Änderung der Dehnung.

$$
\sigma=\eta \frac{d \epsilon}{d t}
$$

Da Gläser beide Arten von Verhalten zeigen, gibt es verschiedene Modelle, die dieses mechanische Verhalten beschreiben. Dafür wird der viskose Anteil durch einen beweglichen Kolben in einer Flüssigkeit der Viskosität $\eta$ beschrieben und der elastische Anteil durch eine Feder der Stärke E. Im Maxwellmodell werden diese beiden in Reihe hintereinander platziert. Dieses Modell beschreibt die Spannungsrelaxation, jedoch wird hier das in viskoelastischen Materialien beobachtete Kriechen nicht erfasst. Dafür kann eine Parallelschaltung von Kolben und Feder, das Kelvin-VoigtModell diesen Effekt beschreiben. Die Abbildung 2.6 zeigt beide Modelle. 


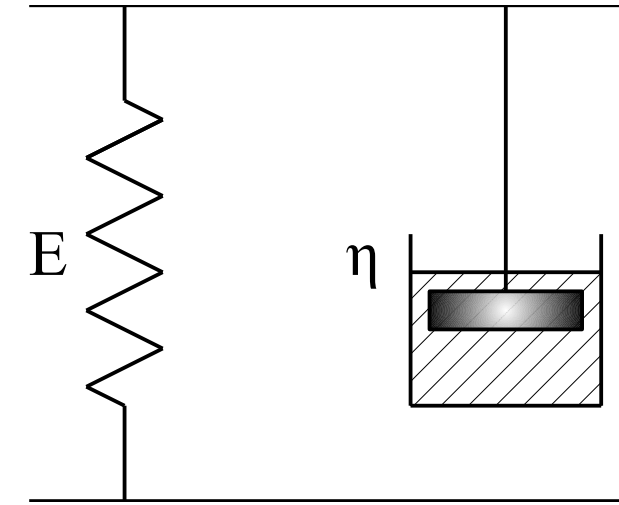

(a) Kelvin-Voigt-Modell

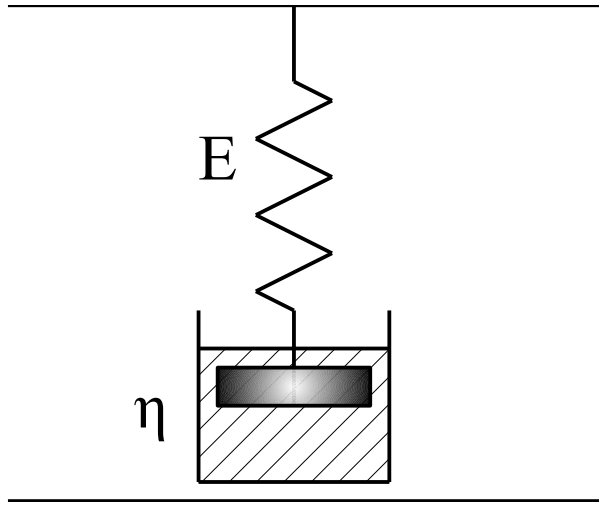

(b) Maxwell-Modell

Abb. 2.6.: Einfache Modelle zur Beschreibung viskoelastischen Verhaltens nach [38]

\subsubsection{Dynamisch mechanische Spektroskopie an viskoelastischen Materialien}

Dynamisch mechanische Spektroskopie (kurz: DMA, Dynamical Mechanical Analysis) ist ein kraftvolles Werkzeug bei der Charakterisierung viskoleastischer Materialien. Da das Material dynamisch beansprucht wird, können zeitabhängige Prozesse direkt beobachtet werden. Typische Messgrößen sind dabei der Speichermodul $E^{\prime}$, der Verlustmodul $E^{\prime \prime}$, sowie die Dämpfung, die über den $\tan \delta$ ausgedrückt wird. Diese Messgrößen und das Prinzip der dynamisch mechanischen Spektroskopie sollen im Folgenden beschrieben werden.

Dynamisch mechanische Spektroskopie beruht im wesentlichen darauf, dass das Probenmaterial einer sich periodisch ändernden externen Dehnung $\epsilon(t)$ ausgesetzt wird und die Antwort des Materials in Form der dafür benötigten zeitabhängigen Spannung beobachtet wird.

$$
\epsilon(t)=\epsilon_{0} \sin (\omega t)
$$

Die zeitabhängige Spannung $\sigma$ zeigt typischerweise ebenfalls einen periodischen Verlauf mit einer Phasenverschiebung.

$$
\sigma(t)=\sigma_{0} \sin (\omega t+\delta)
$$

Häufig wird eine komplexe Darstellung gewählt und es können die komplexe Spannung $\sigma^{*}$ und die komplexe Dehnung $\epsilon^{*}$ definiert werden:

$$
\begin{aligned}
\epsilon^{*}(t) & =\epsilon_{0} \cdot \exp (i \omega t) \\
\sigma^{*}(t) & =\sigma_{0} \cdot \exp (i \omega t-i \delta)=E^{*} \epsilon^{*}(t)=\left(E^{\prime}+i E^{\prime \prime}\right) \epsilon^{*}(t)
\end{aligned}
$$


Dabei bezeichnet $E^{*}$ den komplexen Modul, der sich aus einem elastischen Anteil, der in Phase mit der Anregung ist (E') und einem viskosen Anteil (E") zusammensetzt. Das viskose Verhalten ist durch Verlustprozesse im Material zurückzuführen und führt zu einer Phasenverschiebung $\delta$. Dieser Zusammenhang ist in der Abbildung 2.7 schematisch dargestellt.

Dabei wird $E^{\prime}$ als Speichermodul oder auch Youngsmodul bezeichnet und $E^{\prime \prime}$ als Verlustmodul. Diese beiden Größen sind geometrieabhängig und können mithilfe des Geometriefaktors $g$ aus Anregungskraft $F$ und Auslenkung $d$ bestimmt werden.

$$
\begin{aligned}
& E^{\prime}=E^{*} \cdot \sin \delta=\frac{F}{d} \cdot \frac{1}{g} \cdot \sin \delta \\
& E^{\prime \prime}=E^{*} \cdot \cos \delta=\frac{F}{d} \cdot \frac{1}{g} \cdot \cos \delta
\end{aligned}
$$

Für die Messung kann entweder die Amplitude der dynamischen Spannung vom Gerät vorgegeben werden oder die Amplitude der Dehnung wird konstant gehalten. Typische Messgrößen für beide Messmethoden sind in der Tabelle $2.1 \mathrm{zu}$ sehen (nach [104]). Dabei sind jeweils die elastischen Anteile mit einem Strich und die inelastischen mit zwei Strichen gekennzeichnet. Im Fall einer dehnungskontrollierten Messung, wird die Spannung aufgezeichnet und es können direkt der Speichermodul E' und der Verlustmodul E" bestimmt werden. Für den spannungskontrollierten Fall wird analog die Dehnnachgiebigkeit (Compliance) gemessen. Im linearen Fall ist die Nachgiebigkeit jeweils der Kehrwert des Moduls. Kommen jedoch höhere harmonische Beiträge hinzu, so ist dieser einfache Zusammenhang nicht mehr gegeben.

\begin{tabular}{ll}
\hline Dehnungskontrolliert & Spannungskontrolliert \\
\hline$\epsilon_{0}=$ const & $\sigma_{0}=$ const. \\
$\sigma(t)=\epsilon_{0} \cdot E^{\prime} \sin (\omega t)+\epsilon_{0} \cdot E^{\prime \prime} \cos (\omega t)$ & $\epsilon(t)=\sigma_{0} \cdot C^{\prime} \sin (\omega t)+\sigma_{0} \cdot C^{\prime \prime} \cos (\omega t)$ \\
$E^{*}=\frac{F}{d} \cdot \frac{1}{g}=\frac{\sigma}{\epsilon}=E^{\prime}+i E^{\prime \prime}$ & $C^{*}=\frac{d}{F} \cdot g=\frac{\epsilon}{\sigma}=C^{\prime}+i C^{\prime \prime}$ \\
$E^{\prime}=E^{*} \cos \delta$ & $C^{\prime}=C^{*} \cos \delta$ \\
$E^{\prime \prime}=E^{*} \sin \delta$ & $C^{\prime \prime}=C^{*} \sin \delta$ \\
\hline
\end{tabular}

Tab. 2.1.: Typische Größen der DMA, für dehnungs- und spannungskontrollierte Messungen nach [104]. 


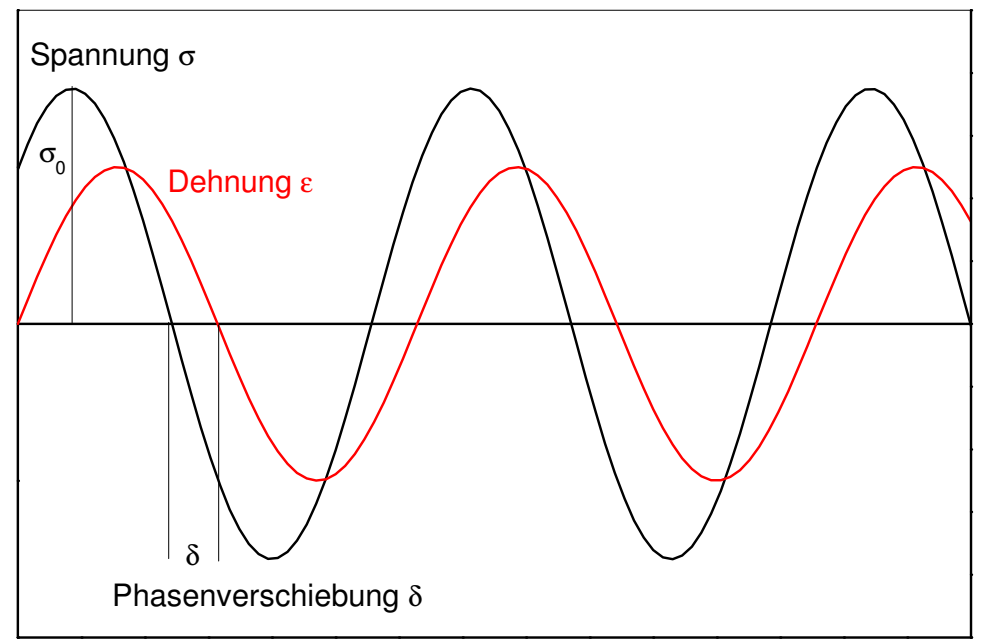

Abb. 2.7.: Der Phasenwinkel zwischen Anregung und Antwort ist ein Maß für den Verlust im Material.

\subsection{Charakteristische Eigenschaften von Polymeren}

\subsubsection{Struktur der Polymere}

Polymere, auch als Makromoleküle bezeichnet, bestehen aus einer großen Anzahl sich wiederholender molekularer Bausteine, den Monomeren, die durch kovalente Bindungen miteinander verbunden sind. Typischerweise bestehen diese Bausteine aus Wasserstoff, Sauerstoff, Stickstoff und Kohlenstoff. Die Anzahl der Monomere wird als Polymerisationsgrad $N$ bezeichnet. Bei den meisten Polymeren, sind die Monomere über Kohlenstoffatome verbunden, so dass sich ein sogenanntes Polymerrückgrat aus Kohlenstoff bildet, von dem aus die Seitenketten abzweigen. Ein Beispiel für ein Monomer ist in Bild 2.8 gezeigt.

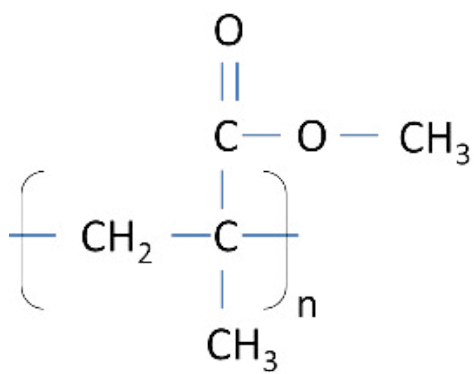

Abb. 2.8.: Monomereinheit von Polymethylmethacrylate (PMMA) 
Die Herstellung von Polymeren liefert keinen einheitlichen Polymerisationsgrad, sondern eine Verteilung. Zur Charakterisierung dieser Verteilung der Molmassen kann einerseits das Gewichtsmittel $\bar{M}_{w}$ und andererseits das Zahlenmittel $\bar{M}_{n}$ bestimmt werden. Das Verhältnis dieser beiden Größen zueinander:

$$
P I=\frac{\bar{M}_{w}}{\bar{M}_{n}}
$$

gibt die Breite der Verteilung an und wird als Polydispersitätsindex bezeichnet [13]. Für eine monodisperse Probe, also Polymermaterial, bei dem alle Ketten den gleichen Polymersationsgrad aufweisen, wäre er genau eins. Besteht ein Polymer aus verschiedenen Monomeren, so wird es als Copolymer bezeichnet. Die verschiedenen Monomere können dabei entweder statistisch angeordnet sein, oder in Blöcken aus jeweils einer Monomersorte, dies wird dann als Blockcopolymer bezeichnet.

\subsubsection{Polymerdynamik}

Die mechanischen Eigenschaften von Polymeren zeigen eine extrem große Bandbreite, welche auf die große Vielfalt an verschiedenen Polymerstrukturen zurückzuführen ist. Dadurch stehen dem Polymer eine Vielzahl verschiedener Bewegungsmöglichkeiten offen und der Frequenzbereich dieser Bewegungen erstreckt sich über einen Bereich von $10^{-3}-10^{14} \mathrm{~Hz}$ [13]. Im Folgenden soll ein Überblick der wichtigsten dieser Polymerbewegungen gegeben werden. Bei sehr kleinen Temperaturen sind nur Molekülschwingungen möglich. Für höhere Temperaturen können dann Segmentbewegungen stattfinden. Dabei kann es sich beispielsweise um eine Rotation von Seitenketten handeln [85]. Bei diesen sekundären Bewegungsvorgängen bleibt die Konformation der Hauptkette jeweils nahezu konstant. Da aufgrund der großen Unterschiede in der Polymerstruktur sehr verschiedene Relaxationen auftreten können, sollen hier nur einige am Beispiel von PMMA kurz behandelt werden. Die Abbildung 2.9 zeigt die Temperaturabhängigkeit des Verlustmoduls. Es werden neben der $\alpha$-Relaxation 3 weitere Peaks beobachtet. Dabei konnte festegestellt werden, dass der $\beta$-Peak durch Seitenkettenbewegungen der Ester-Gruppen hervorgerufen wird, während bei kleineren Temperaturen die Bewegung von Methylgruppen der Hauptkette $(\gamma)$ und der Seitenketten $\delta$ zu einem deutlichen Peak im Verlust führen [41].

Der bedeutendste Übergang ist jedoch der Glasübergang, der als $\alpha$-Peak bezeichnet wird. Als mikroskopische Ursache wird hier eine Bewegung der Hauptkette gesehen, die sich in erster Näherung mithilfe des Rouse-Modells beschreiben läßst [78]. Das Rouse-Modell beschreibt ein Polymer anhand von Massepunkten, sogenannten Perlen, die mit Federn verbunden sind. Diese Rousesegmente unterliegen einereseits der Brownschen Bewegung aufgrund von thermischer Anregung und andereseits einer zusätzlichen stochastischen Verteilung von Kräften $\left(f_{n}(t)\right)$. Dies führt zu einer 


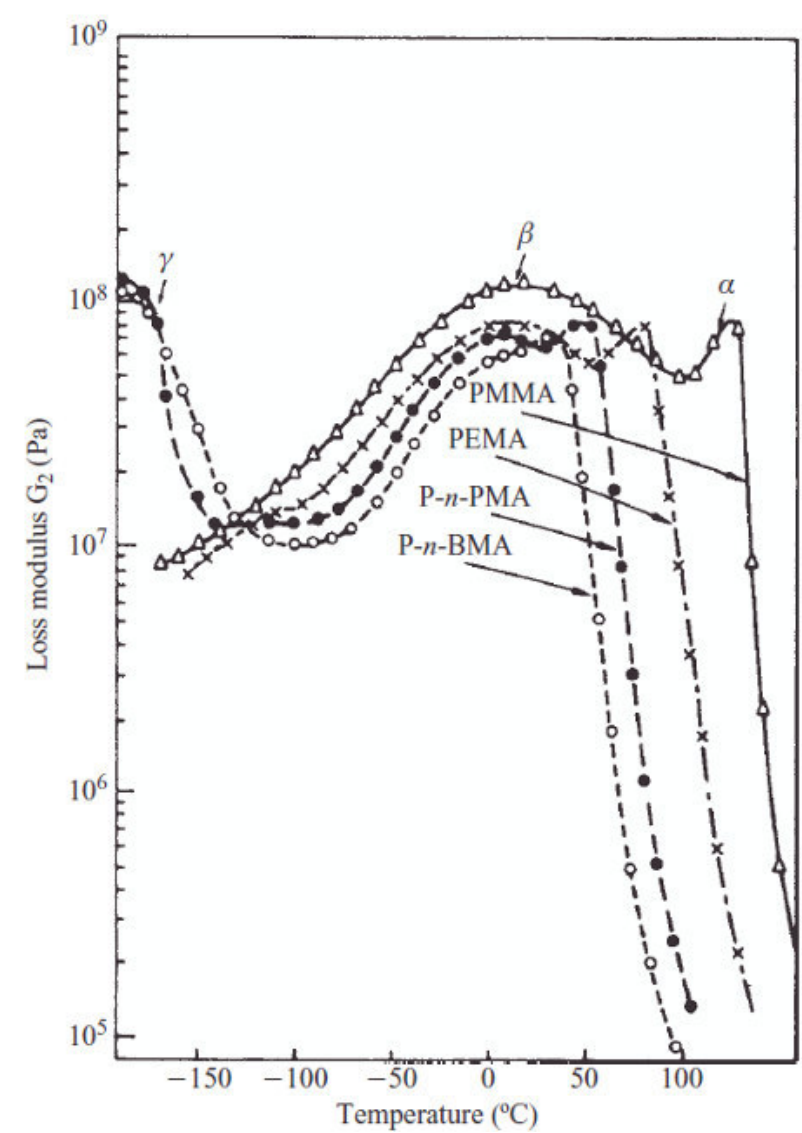

Abb. 2.9.: Temperaturabhängigkeit des Verlustmoduls für verschiedene Polymere [41].
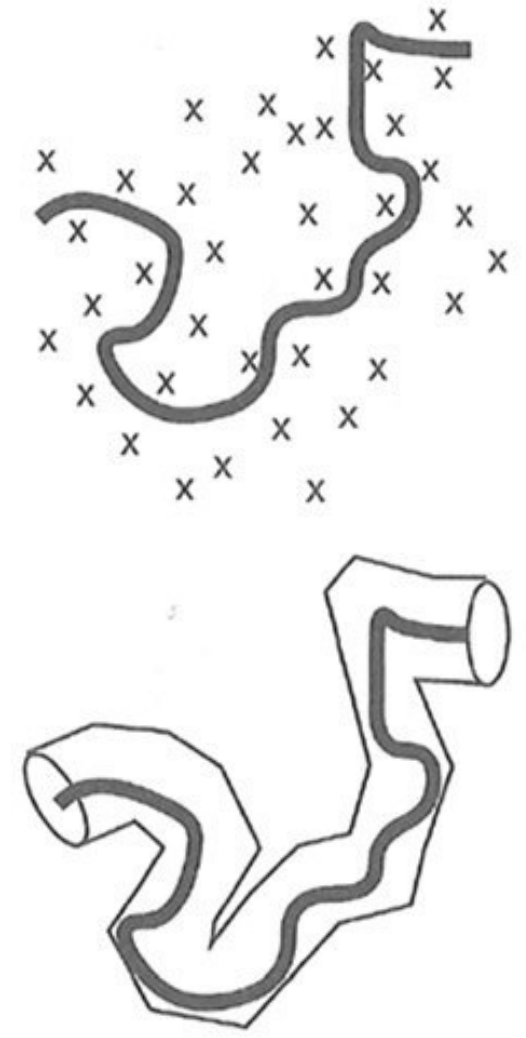

Abb. 2.10.: Schema der Reptation eines Poylmers [56]

Langevin-Gleichung für jedes Segment $n$ [43]:

$$
\zeta \frac{d \vec{r}_{n}}{d t}=\frac{3 k_{B} T}{a^{2}}\left(\vec{r}_{n+1}-2 \vec{r}_{n}\right)+\vec{f}_{n}
$$

Dabei stellt $\zeta$ den Reibungskoeffizeint pro Segment und $a$ die Segmentlänge dar. Daraus kann schließlich die folgende Relaxationszeit ermittelt werden:

$$
\tau_{R}=\frac{\xi N^{2} a^{2}}{3 \pi^{2} k_{B} T}
$$

Bei diesem Modell sind jedoch einige wichtige Eigenschaften von Polymeren nicht berücksichtigt. So werden Volumenwechselwirkungen und topographische Wechselwirkungen der Ketten untereinander ausgeschlossen, so dass sich im Modell die Ketten durchdringen können. Damit ist das Rouse-Modell geeignet die Kurzzeitdynamik in Polymerschmelzen gut zu beschreiben, da hier noch keine großskaligen Bewegungen der Kette erfasst werden. Für längere Zeiten wird erwartet, dass es zu Fließprozes- 
sen kommt und daher Volumenauschlusseffekte wichtig werden. Dies wird durch das nachfolgend beschriebene Reptationsmodell erfasst [35], [24]. Dieses Modell ist besonders geeignet, den Fließsprozess in verknäulten Polymerschmelzen zu beschreiben. Aufgrund der Verknäulung der Polymere untereinander, kann eine einzelne Polymerkette seine Position nur verändern, indem sie sich zwischen den anderen Poylmeren hindurchschlängelt. Zur Beschreibung dieser Schlängelbewegung (Reptation) wird zunächst eine Röhre definiert, durch die sich das Polymer bewegt. Diese Röhre wird gebildet durch die Begrenzungen der anderen Poylmere. In der Abbildung 2.10 ist dies schematisch dargestellt. Da die Anzahl der Verschlaufungspunkte grundlegend durch die Molmasse der Polymere bestimmt ist, kann mit diesem Modell der Zusammenhang von Relaxationszeit und Molmasse der Polymere bestimmt werden. Dabei wird in Übereinstimmung mit experimentellen Ergebnissen [27] der Zusammenhang $\tau \propto M^{3}$ gefunden.

\subsubsection{Blockcopolymere und Mikrophasenseparation}

Blockcopolymere bestehen aus zwei oder mehr Blöcken einer Monomersorte. Sind nur zwei verschiedene Monomere A und B vertreten, so wird das Polymer als Diblockcopolymer bezeichnet. Neben dem Poymerisationsgrad sind diese Polymere vor allem durch die Zusammensetzung $f$ gekennzeichnet. Dabei bezeichnet $f$ den Volumenanteil der Komponente A des Polymers. Damit kann für die beiden Blöcke jeweils ein spezifischer Polymerisationsgrad $N_{A}=f N$ beziehungsweise $N_{B}=(1-f) N$ angegeben werden. Von besonderem Interesse ist die Wechselwirkung zwischen den Blöcken, die die Mischbarkeit der beiden Blöcke bestimmt. Diese Mischbarkeit wird durch den temperaturabhängigen Flory-Huggins Parameter $\chi$ beschrieben. Besteht eine Tendenz zur Entmischung, so können sich jedoch die kovalent aneinander gebundenen Blöcke nicht makroskopisch entmischen, sondern dies nur im Bereich ihrer Größenordnung tun. Dies führt zur Bildung von sogenannten Mikrophasen. Die Abbildung 2.11 veranschaulicht dies schematisch. Mit steigender Temperatur sinkt jedoch der Flory Huggins Parameter und es kann zu einer Mischung der Phasen kommen. Diesen temperaturgetriebenen Übergang bezeichnet man als OrdnungsUnordnungs-Übergang (kurz : ODT von engl. Order to Disorder Transition).

Abhängig von der Zusammensetzung $f$ des Blockcopolymers werden eine Vielzahl verschiedener Morphologien der Mikrophasen beobachtet. Diese Morphologien können beispielsweise Lamellen, geordnete Zylinder oder Kugeln auf einem bcc-Gitter sein. Besonders etabliert zur Bestimmung der Oberflächenmorphologie ist die Rasterkraftmikroskopie, die im Abschnitt 3.5 behandelt wird. Die Abbildung 2.12 zeigt ein theoretisches Phasendiagramm nach Bates. Aufgrund der unterschiedlichen Strukturen können auch verschiedene mechanische Eigenschaften der Phasen beobachtet werden.

Für binäre Polymermischungen können anhand der Werte von $T_{g}$ zusätzlich Aussagen über die Kompatibilität der Mischungen getroffen werden. Eine perfekte Misch- 


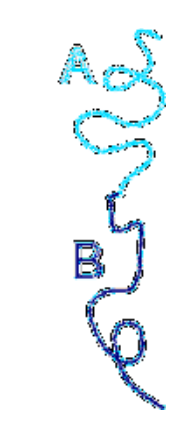

Diblockcopolymer

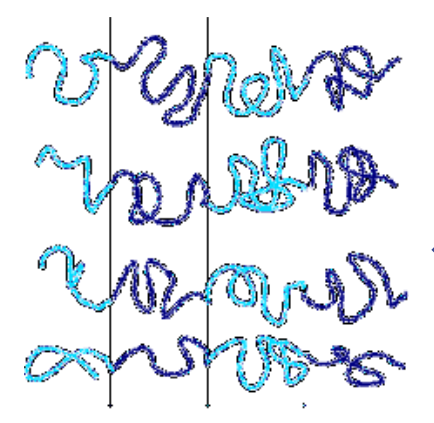

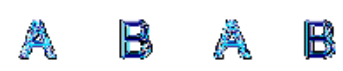

geordnet

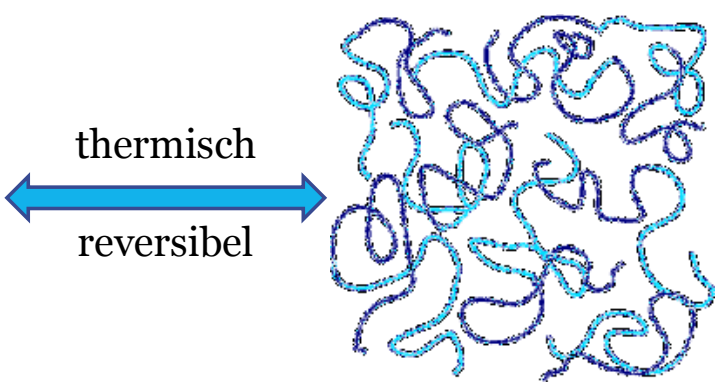

ungeordnet

Abb. 2.11.: Ein Diblockcopolymer kann nur auf Längenskalen entsprechend der Polymerlänge entmischen. Mit steigender Temperatur wird die Entropie erhöht, so dass es zu einem ungeordneten Zustand kommt. Nach [6]

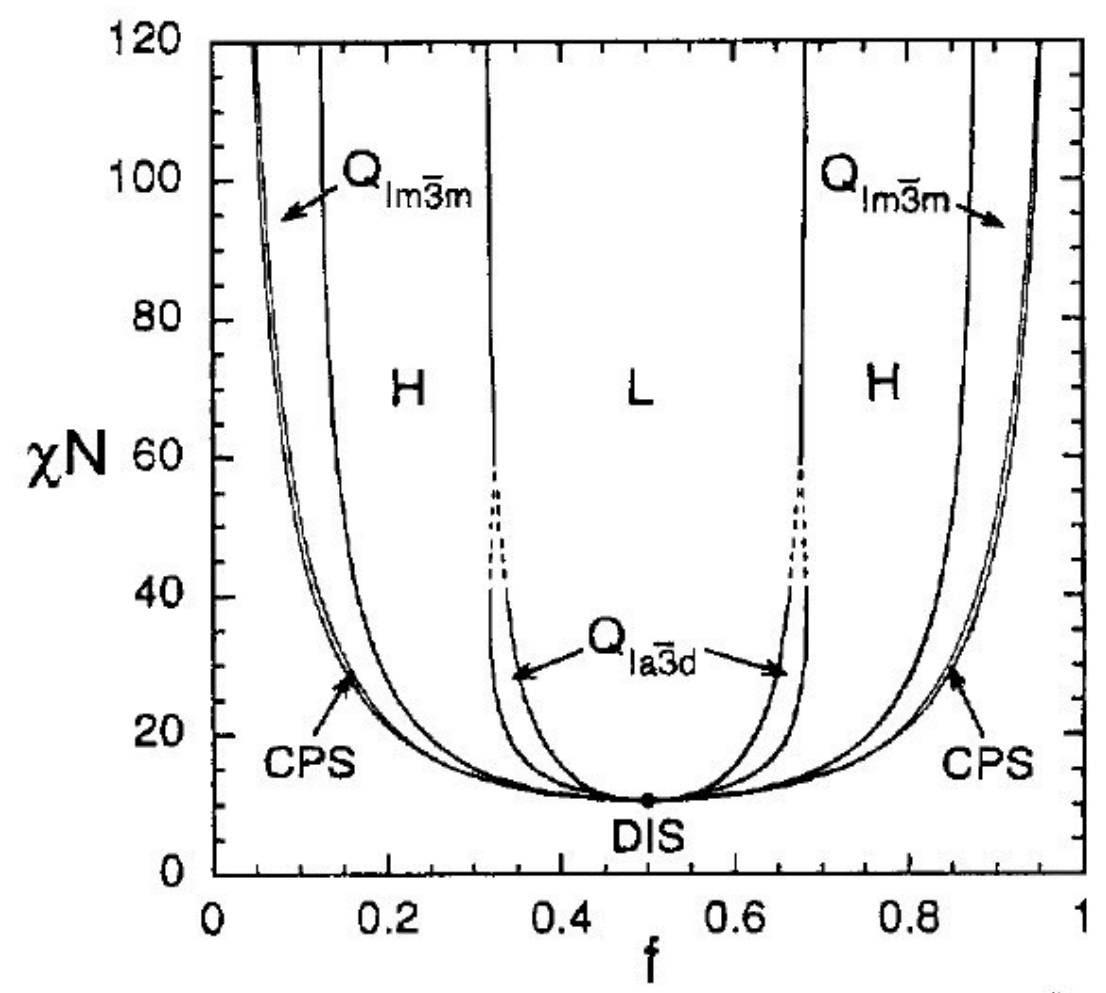

Abb. 2.12.: Theoretisches Phasendiagramm für ein symmetrisches Blockcopolymer nach [64]. 
barkeit zeigt sich in diesem Fall durch die Beobachtung einer einzigen Glastemperatur. Für nicht mischbare Systeme werden zwei einzelne Glastemperaturen erwartet, die mit der Komposition $f$ der Zusammensetzung variieren [15].

Aus den mechanischen Daten der in dieser Arbeit untersuchten Blockcopolymere können jedoch wenig Rückschlüsse auf die vorliegenden Morphologien der untersuchten Probensysteme geschlossen werden. Das hier untersuchte BCP besteht nämlich aus einem weichen BA-Block und einem harten PMMA-Block, so dass für Proben verschiedener Zusammensetzung auch ohne Berücksichtung der Morphologie eine signifikante Änderung der mechanischen Eigenschaften erwartet wird. Daher können Proben verschiedener Zusammensetzung nicht anhand ihrer globalen mechanischen Eigenschaften bestimmten Morphologien zugeordnet werden. Stattdessen kann mithilfe des Rasterkraftmikroskops anhand der Oberflächentopologie die Morphologie bestimmt werden. Falls ein Übergang jedoch von einer Struktur zu einer anderen mithilfe der Temperatur erreicht wird, so ist dies direkt in den mechanischen Eigenschaften zu beobachten.

\subsection{Nichtlineares Verhalten amorpher Materie}

In diesem Abschnitt soll kurz in das nichtlineare Verhalten amorpher Materialien eingeführt werden. Zunächst wird ein Überblick gegeben, wann nichtlineares Verhalten auftritt und die zu erwartetenden nichtlinearen Beiträge werden eingeführt. Anschließend wird das Box-Modell vorgestellt, welches erfolgreich die nichtlinearen Effekte für einige Experimente der dielektrischen Spektroskopie erklärt. Am Ende wird die Verknüpfung der nichtlinearen Suszeptibilitäten mit $N_{\text {corr }}$, der Zahl der korrelierten Teilchen im Glas vorgestellt.

\subsubsection{Nichtlineare Antwort}

Typische Versuche an amorphen Materialien beschränken sich auf den Bereich kleiner externer Felder, so dass eine lineare Beschreibung, bei der die Antwort des Materials proportional zum äußeren Feld ist, das Materialverhalten gut wiedergibt. In Anwendungen werden Materialien häufig jedoch solch großen Spannungen ausgesetzt, bei denen die sich die Materialantwort nicht mehr durch einen einfachen linearen Zusammenhang beschreiben lässt. Desweiteren können durch hohe Anregungsspannungen zusätzliche mikroskopische materialpsezifische Eigenschaften der Probe sichtbar werden. Beispielsweise kann bei Polymeren Information über die Topologie und die Verzweigung gewonnen werden. Insbesondere ist jedoch die nichtlineare Antwort in amorphen Materialen als Folge ihrer komplexen langreichweitigen Struktur zu verstehen und kann daher weiteren Aufschluss über die komplexe Dynamik der Flüssigkeiten geben.

Neben der Anregungsamplitude haben vor allem die äußeren Bedingungen unter de- 


\section{Theoretische Grundlagen}

nen ein Experiment abläuft Einfluss darauf, ob nichtlineares Verhalten beobachtet wird. Diese äußeren Parameter sind beispielsweise Temperatur und Druck. Sie beeinflussen die materialspezifische Relaxationszeit $\tau_{0}$, in welcher das Material vollständig relaxieren kann. Daneben ist auch die charakteristische Beobachtungszeit des Experimentes $t_{b e}$ von Bedeutung und kann mit der Relaxationszeit über die Deborahzahl $D e=\frac{\tau_{0}}{t_{b} e}$ verknüpft werden. Große Werte von $D e$ werden für kurze Beanspruchung oder lange Relaxationszeiten erreicht, so dass für diese Experimente ein elastisches Verhalten beobachtet wird. Experimente mit Deborahzahlen nahe Null beobachten den Fließsprozess eines Materials. Das bedeutet das Material kann innerhalb der Beobachtungszeit vollständig relaxieren und verhält sich damit wie eine Newtonsche Flüssigkeit. Dies tritt auf, wenn die Relaxation entweder sehr schnell ist oder das Experiment entsprechend lange dauert. Für $D e \approx 1$ tritt das in Abschnitt 2.2.1 behandelte viskoelastische Verhalten auf. Wird die Deborahzahl in Zusammenhang zur externen Anregungsamplitude gesetzt, so ergibt sich das von Pipkin entwickelte Diagramm (Abblidung 2.13) anhand dessen eine Abschätzung getroffen werden kann, ob sich ein Material in einem Experiment nichtlinear verhält.

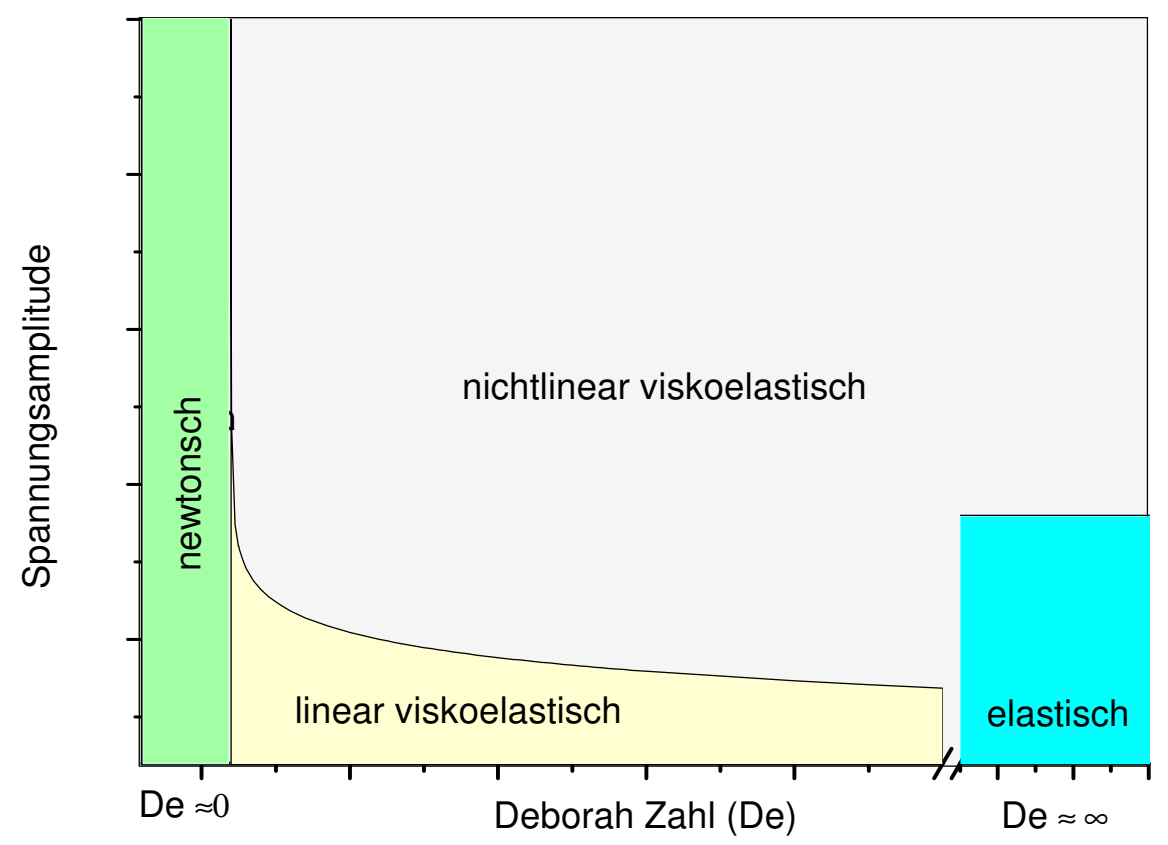

Abb. 2.13.: Mechanisches Verhalten von Materialien in Abhängigkeit der Deborahzahl und der Spannungsamplitude. [68]

Ein typisches Experiment, um komplexe Flüssigkeiten im nichtlinearen Bereich zu untersuchen, ist das Anlegen eines äußeren periodischen Feldes mit großer Amplitude. Die hohe Amplitude sorgt dafür, dass man sich im oberen Teil des Diagrammes 
bewegt und das Material nichtlineares Verhalten zeigt. Mithilfe des periodischen Feldes können direkt, die in Abschnitt 2.2.2 behandelten, dynamischen Größen untersucht werden. Im Folgenden sollen die benötigten theoretischen Grundlagen zur Beschreibung des nichtlinearen Verhaltens in Anlehnung an [16] bereitgestellt werden. Die Formeln werden für die in dieser Arbeit verwendeten dynamisch mechanischen Spektroskopie angegeben. Sie sind jedoch für andere Spektroskopiearten analog zu formulierern.

Ein Material werde einer äußeren mit der Frequenz $\omega$ oszillierenden Spannung $\sigma(t)$ ausgesetzt.

$$
\sigma(t)=\sigma_{0} \cdot \cos (\omega t)
$$

Das Material wird darauf mit einer zeitabhängigen Dehnung reagieren, die sich nach dem externen Spannungsfeld entwickeln läßt. Für einen symmetrischen Messaufbau, bei dem die Spannungsoszillation um die Nullinie stattfindet $(|\sigma(t)|=|\sigma(t+\pi)|$ muss die Antwortfunktion eine ungerade Funktion sein, so dass keine geraden Terme erlaubt sind.

$\epsilon(t)=\int_{-\infty}^{\infty} C_{1}\left(t-t^{\prime}\right) \sigma\left(t^{\prime}\right) d t^{\prime}+\iiint_{-\infty}^{\infty} C_{3}\left(t-t_{1}^{\prime}, t-t_{2}^{\prime}, t-t_{3}^{\prime}\right) \times \sigma\left(t_{1}^{\prime}\right) \sigma\left(t_{2}^{\prime}\right) \sigma\left(t_{3}^{\prime}\right) d t_{1}^{\prime} d t_{2}^{\prime} d t^{\prime} 3+\ldots$

Dabei ist $C_{1}$ die lineare Nachgiebigkeit und $C_{3}$ die kubische nichtlineare Nachgiebigkeit in der Zeitdomäne. Der Übersicht halber wird die Entwicklung nach dem kubischen Term abgebrochen, sie kann jedoch analog weitergeführt werden für Terme höherer Ordnung. Mit dem Feld aus Gleichung 2.21 kann die Dehnung in Gleichung 2.22 fouriertransformiert werden und es ergibt sich folgender Zusammenhang in der Frequenzdomäne [16]:

$\epsilon(t)=\sigma_{0}\left|\hat{C}_{1}\right| \cos \left(\omega t-\delta_{1}\right)+\frac{3}{4} \sigma_{0}^{3}\left|\hat{C}_{1}^{(3)}\right| \cos \left(\omega t-\delta_{1}^{(3)}\right)+\ldots+\frac{1}{4} \sigma_{0}^{3}\left|\hat{C}_{3}^{(3)}\right| \cos \left(3 \omega t-\delta_{3}^{(3)}\right)+\ldots$

Es zeigt sich, dass es zwei verschiedene Nachgiebigkeiten dritter Ordnung gibt. Beide sind proportional zu $\sigma_{0}^{3}$, jedoch treten sie bei jeweils verschiedenen Frequenzen auf. $C_{1}^{(3)}$ ist der Beitrag dritter Ordnung bei der fundamentalen Frequenz, während $C_{3}^{(3)}$ bei der 3. Harmonischen auftritt.

Damit ergeben sich für die Dehnungsantwort bei der fundamentalen Frequenz $\omega$ und bei der dritten harmonischen Frequenz $3 \omega$ jeweils die folgenden Dehnungsamplituden $\hat{\epsilon}_{1}$ und $\hat{\epsilon}_{3}$ (nach [16],[72]):

$$
\begin{aligned}
& \hat{\epsilon}_{1}=\hat{C}_{1} \sigma_{0}+\frac{3}{4} \hat{C}_{1}^{(3)} \sigma_{0}^{3}+\frac{5}{8} \hat{C}_{1}^{(5)} \sigma_{0}^{5}+\frac{35}{64} \hat{C}_{1}^{(7)} \sigma_{0}^{7}+\ldots \\
& \hat{\epsilon}_{3}=\frac{1}{4} \hat{C}_{3}^{(3)} \sigma_{0}^{3}+\frac{5}{16} \hat{C}_{3}^{(5)} \sigma_{0}^{5}+\frac{21}{64} \hat{C}_{3}^{(7)} \sigma_{0}^{7}+\ldots
\end{aligned}
$$




\section{Theoretische Grundlagen}

Zunächst soll die Antwortfunktion bei der fundamentalen Frequenz $\hat{\epsilon_{1}}$ betrachtet werden. Der dominierende Term ist dabei die Nachgiebigkeit $C_{1}$, wird diese in Imaginär- und Realteil zerlegt, so ergeben sich die aus der linearen Beschreibung bekannten Größen $C^{\prime}$ (Speichernachgiebigkeit) und $C^{\prime \prime}$ (Verlustnachgiebigkeit).

Soll die nichtlineare Antwort bei der Fundamentalen direkt mit dem linearen Fall verglichen werden, so kann ein $\Delta \ln \hat{C}$ definiert werden, welches den nichtlinearen Beitrag zur fundamentalen Antwort beschreibt.

$$
\begin{aligned}
\hat{\epsilon_{1}} & =\hat{C}_{1} \sigma_{0}+\frac{3}{4} \hat{C}_{1}^{(3)} \sigma_{0}^{3}=\hat{C}_{1} \sigma_{0}(1+\underbrace{\frac{3}{4} \lambda_{1}^{(3)} \sigma_{0}^{2}}_{\Delta \ln C})=\hat{C}_{1}^{n l}(\sigma) \sigma_{0} \\
& \Rightarrow \Delta \ln \hat{C}(\sigma)=\frac{C_{1}^{n l}(\sigma)-\hat{C}_{1}}{\hat{C}_{1}}
\end{aligned}
$$

Damit kann $\Delta \ln \hat{C}(\sigma)$ bestimmt werden, indem zunächst im linearen Bereich bei kleinen Anregungen $\hat{C}_{1}$ gemessen wird. Anschließend wird bei großer Spannungsamplitude im nichtlinearen Bereich gemessen, so dass die Nachgiebigkeit $\hat{C}_{1}^{n l}$ erhalten wird, welche zusätzlich nichtlineare Beiträge enthält. Es wird erwartet, dass $\Delta \ln \hat{C}$ mit dem Qaudrat der Anregungsamplitude skaliert.

Die andere Herangehensweise versucht die Quantifizierung der nichtlinearen Effekte über die Beiträge bei den höheren harmonischen Frequenzen. Zunächst wird dabei stets die Antwort bei der 3.Harmonischen untersucht, da diese den größten Beitrag liefert. Jedoch können auch weitere höhere Harmonische ausgewertet werden, wenn deren Intensität ausreichend ist.

Vergleicht man die Amplitude bei der fundamentalen Frequenz mit der der Amplitude der 3. Harmonischen, so ergibt sich:

$$
\frac{\hat{\epsilon_{3}}}{\hat{\epsilon_{1}}}=\frac{\frac{1}{4} \hat{C}_{3}^{(3)} \sigma_{0}^{3}}{\hat{C}_{1} \sigma_{0}+\frac{3}{4} \hat{C}_{1}^{(3)} \sigma_{0}^{2}}=\frac{\frac{1}{4} \hat{C}_{1} \lambda_{3}^{(3)} \sigma_{0}^{3}}{\hat{C}_{1} \sigma_{0}(1+\underbrace{\frac{3}{4} \lambda_{1}^{(3)} \sigma_{0}^{2}}_{\Delta \ln C})}
$$

Es werden folglich die nachfolgenden Zusammenhänge im Experiment erwartet. Für die Antwort bei der fundamentalen Frequenz wird zunächst ein linearer Verlauf beobachtet, welcher bei großen Amplituden von diesem Verhalten abweicht. Diese Abweichung kann auf einen zusätzlichen Beitrag $\Delta \ln \hat{C}$ zurückgeführt werden. Dieser Beitrag soll mit dem Quadrat der Spannungsamplitude $\sigma_{0}^{2}$ wachsen. Für die Dehnungsantwort bei der dritten Harmonischen wird erwartet, dass diese mit $\sigma_{0}^{3}$ wächst. In Gleichung 2.28 ist das Verhältnis der Antwort bei der dritten Harmonischen und der Fundamentalen dargestellt. In erster Näherung kann der Beitrag $\Delta \ln C$ vernachlässigt werden, da er im Vergleich zu $\hat{C}_{1} \sigma_{0}$ ungefähr nur $1 \%$ ausmacht. Damit ergibt 
sich für das Verhältnis der Amplituden.

$$
\frac{A_{3}}{A_{1}} \propto \frac{\sigma_{0}^{3}}{\sigma_{0}^{1}}=\sigma_{0}^{2}
$$

Also wird eine Abhängigkeit von $\sigma_{0}^{2}$ erwartet.

\subsubsection{Box Modell und dielektrische Spektroskopie}

Im Folgenden soll im Zusammenhang der dielektrischen Spektroskopie ein Modell für das Auftreten nicht linearer zeitabhängiger Beiträge, das sogenannte „Box Modell", vorgestellt werden. Die dielektrische Spektroskopie steht mit der mechanischen Spektroskopie in einem engen Zusammenhang. Typischerweise wird hierbei die Polarisation einer Probe als Antwort auf ein elektrisches Wechselfeld in Abhängigkeit dessen Frequenz bestimmt. Besonders hervorzuheben ist hierbei der große Frequenzbereich von $10^{-6}$ bis $10^{12} \mathrm{~Hz}$, der experimentell zugänglich ist. Dies führt dazu, dass die in amorphen Materialien auftretenden molekularen Bewegungen hinsichtlich ihrer Zeitskalen sehr detailiert untersucht werden können.

Vor allem in Hinblick auf das Konzept der dynamischen Heterogenitäten, als Ursache für das beobachtete gestreckt exponentielle Relaxationsverhalten, konnte die dielektrische Spektroskopie einen experimentellen Nachweis liefern [81]. Für diesen Nachweis wurde die Methode des dielektrischen Lochbrennens verwendet. Dabei wird jeweils ein Subensemble des Materials selektiert, indem mit einer bestimmten Frequenz angeregt wird. Anschließend wird das Absorptionsspektrum der Probe gemessen und die dadurch entstandene Veränderung $\Delta \epsilon^{\prime \prime}$ des Verlustspektrums beobachtet. Die Abbildung 2.14 zeigt die erwarteten Absorptionsspektren für den Fall, dass das Spektrum auf eine heterogene Verteilung oder auf eine homogene Verteilung zurückzuführen ist. Dabei wird für das homogene Szenario lediglich eine Verschiebung des gesamten Spektrums erwartet. Für den heterogenen Fall hingegen ensteht bei der Frequenz der angeregten Mode ein Loch, da diese aufgrund der aufgenommenen Energie nun eine beschleunigte Dynamik besitzt und damit nun bei höherer Frequenz beiträgt (inverses Loch).

Im Kontext des dielektrischen Lochbrennens wurde das Box-Modell entwickelt. Dabei werden im System einzelne Boxen definiert, die unabhängig voneinander dynamisch relaxieren und dabei ein Debye-Verhalten mit einer jeweils eigenen Relaxationszeit $\tau_{i}$ zeigen. Wird nun ein starkes elektrisches Feld $\left(E=E_{0} \sin (\omega t)\right)$ angelegt, so wird Energie im Material dissipiert. Dabei nimmt jedoch jede der Boxen abhängig von ihrem $\tau_{i}$ eine unterschiedliche Energiemenge auf. Diese selektiv aufgenomenne Energiemenge wird in Form einer lokalen fiktiven Temperatur $T_{f, i}$ erfasst. Gleichzeitig bedeutet eine Zunahme der fiktiven Temperatur eine schnellere Relaxationszeit und damit eine andere Position im experimentellen Absorptionsspektrum.

Dies wird in Abbildung 2.15 schematisch für 5 Moden und ein elektrisches Feld der 

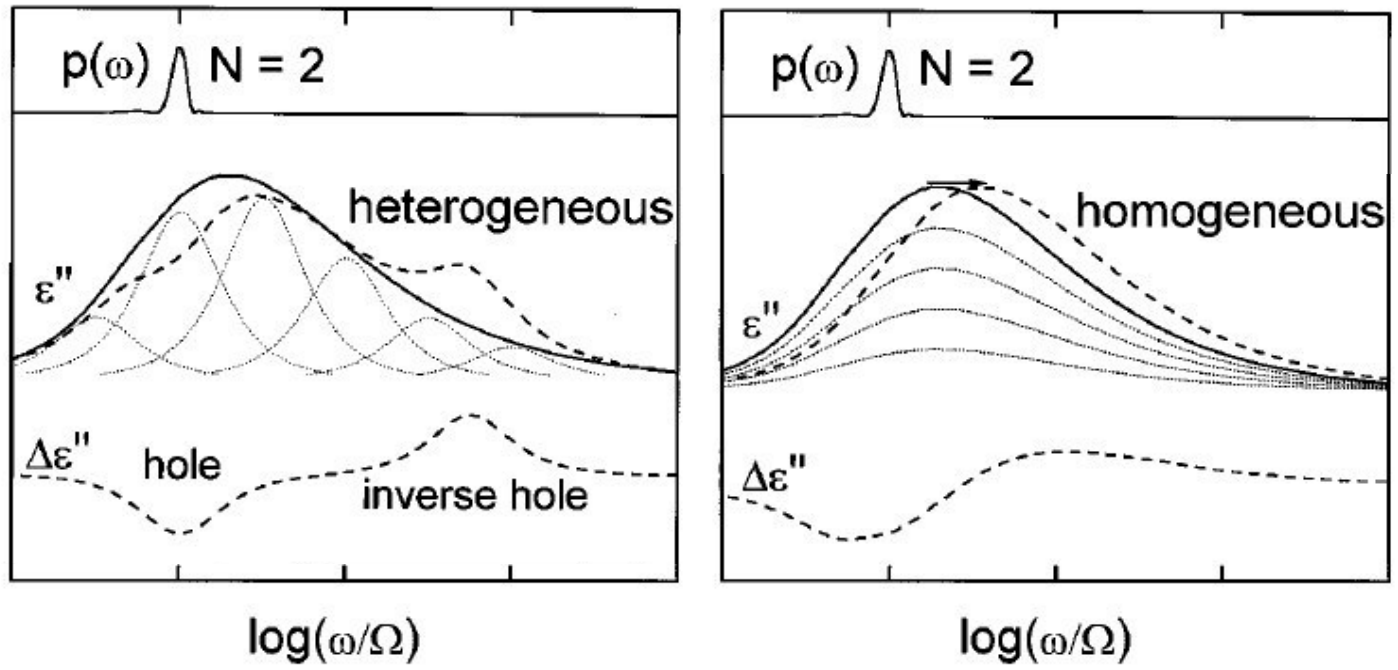

Abb. 2.14.: Darstellung des Prinzips des dielektrischen Lochbrennens zur Unterscheidung eines homogenen und eines heterogenen Szenarios als Ursache für das $A b$ sorptionsspektrum [81].

Frequenz $\omega$ gezeigt. Die kleinen Pfeile an den einzelnen Verlustpeaks der Moden symbolisieren dabei, wie weit jeweils eine Mode durch die Anregung verschoben wird. Dabei zeigen die langsamen Moden eine stärkere Verschiebung, da sie mehr Energie akkumulieren, die sie erst nach ihrer spezifischen Relaxationszeit $\tau_{i}$ ihre Energie an das Phononenbad weitergeben können [46]. Damit wird die Zeitskala, bis das System nach einer spektralen Anregung wieder im Gleichgewicht ist, also die einzelnen Moden ihre Energie abgegeben haben, durch die langsamen Moden bestimmt [97]. Dieses Modell konnte bisher erfolgreich das nichtlineare Verhalten des dielektrischen Verlustes bei hohen Feldern beschreiben [77], [45].

\subsubsection{Kooperativität und höhere nichtlineare Suszeptibilitäten}

Das im vorangegangenen Abschnitt vorgestellte Box-Modell geht zwar von dynamischen Heterogenitäten (den Boxen) aus, trifft jedoch keinerlei Aussagen über die Größe oder die räumliche Verteilung dieser Boxen. Ein anderer Ansatz ist das Konzept der dynamischen Heterogenitäten, nach welchem die Relaxationen direkt durch kollektive Bewegungen von Molekülgruppen bestehend aus der Anzahl $N_{\text {corr }}$, verursacht werden [25], [75],[84]. Desweiteren wird der in Abschnitt 2.1.3 beschriebene Effekt, der stark ansteigenden Viskosität bei Annäherung an $T_{g}$ aus der Schmelze, auf einen Anstieg der Zahl der korrelierten Moleküle $N_{\text {corr }}$ zugeschrieben. Daher ist die Temperaturabhängigkeit von $N_{\text {corr }}$ ein direkter Zugang zum Verständnis des Glasüberganges. Ein großer Beitrag dazu wurde von Bouchaud und Biroli gelie- 

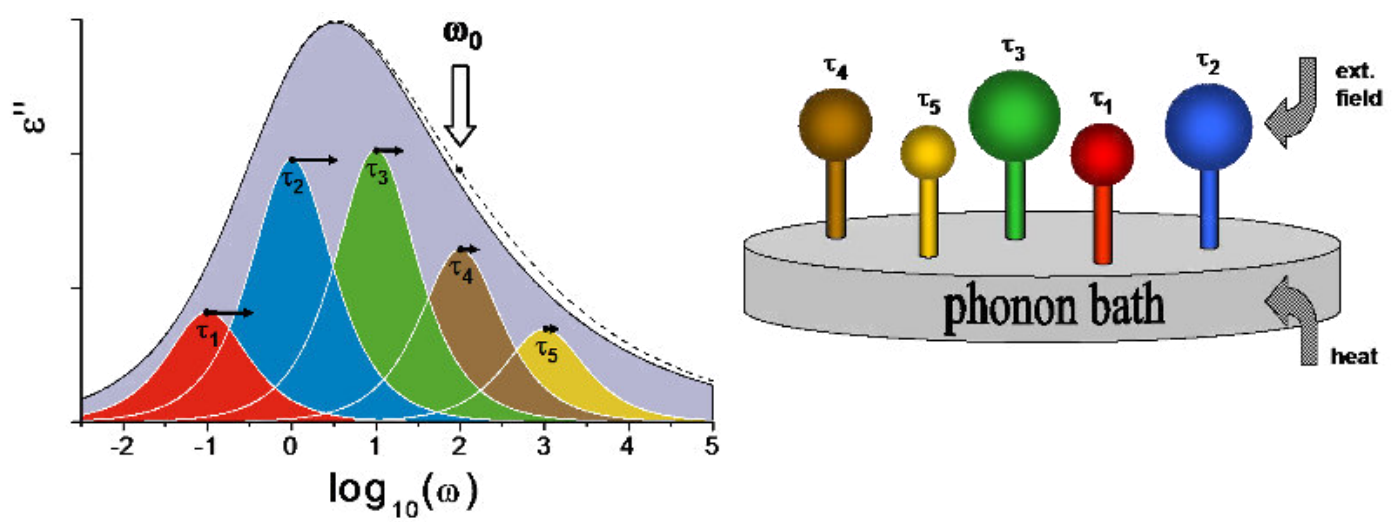

Abb. 2.15.: Schematische Darstellung der spektralen (links) und der räumlichen (rechts) Verteilung der Relaxationszeiten in einem System mit hetereogener Dynamik. Die Dispersion wird durch 5 unterschiedliche Zeitkonstanten beschrieben $\tau_{1}$ bis $\tau_{5}$ Die räumliche Darstellung verdeutlicht, dass eine relativ schnelle Mode wahrscheinlich von viel langsameren Moden begrenzt ist. [74]

fert [12]. Sie konnten eine proportionale Abhängigkeit von $N_{\text {corr }}$ zur nichtlinearen Suszeptibilität $\chi_{3}$ zeigen. Da die langreichweitige Ordnung in amorphen Materialien typischerweise nicht durch eine Zweipunkt-Korrelationsfunktion erfasst werden kann, sondern es sich vielmehr um eine quadrupolare Struktur handelt, kann erst eine Korrelationsfunktion höherer Ordnung diese Struktur beschreiben. Indem die nichtlineare Antwort untersucht wird, kann jedoch Information über Beiträge höherer Ordnung erhalten werden. Zudem konnten Bouchaud et al. voraussagen, dass diese nichtlineare Antwort auf typischen Frequenzen $1 / \tau_{\alpha}$, die direkt mit der strukturellen Relaxationszeit $\tau_{\alpha}$ verknüpft sind, auftritt. Erste experimentelle Arbeiten zeigen, dass die nichtlineare dielektrische Suszeptibilität einen Peak für $\chi_{3}$ zeigt [91], [19], [7]. Die Abbildung 2.16 zeigt das Ergebnis einer dieser Messungen. Es wurde für verschiedene Temperaturen oberhalb von $T_{g}$ jeweils für einen Frequenzbereich von $10^{-3} \mathrm{~Hz}$ bis $10^{2} \mathrm{~Hz}$ die nichtlineare dielektrische Suszeptibiltät $\chi_{3}$ aufgetragen. Dabei zeigt $\chi_{3}$ jeweils ein breites Maximum. Die Temperaturabhängigkeit des Maximums ist im Inset gezeigt und ist direkt proportional zu $N_{\text {corr }}$. Da der verwendete DMA eine zu geringe spektrale Auflösung hat, kann dieser Peak in den mechanischen Spektren nicht eindeutig beobachtet werden. Insbesondere der Bereich oberhalb von $T_{g}$ ist experimentell nicht zugänglich. 
2. Theoretische Grundlagen

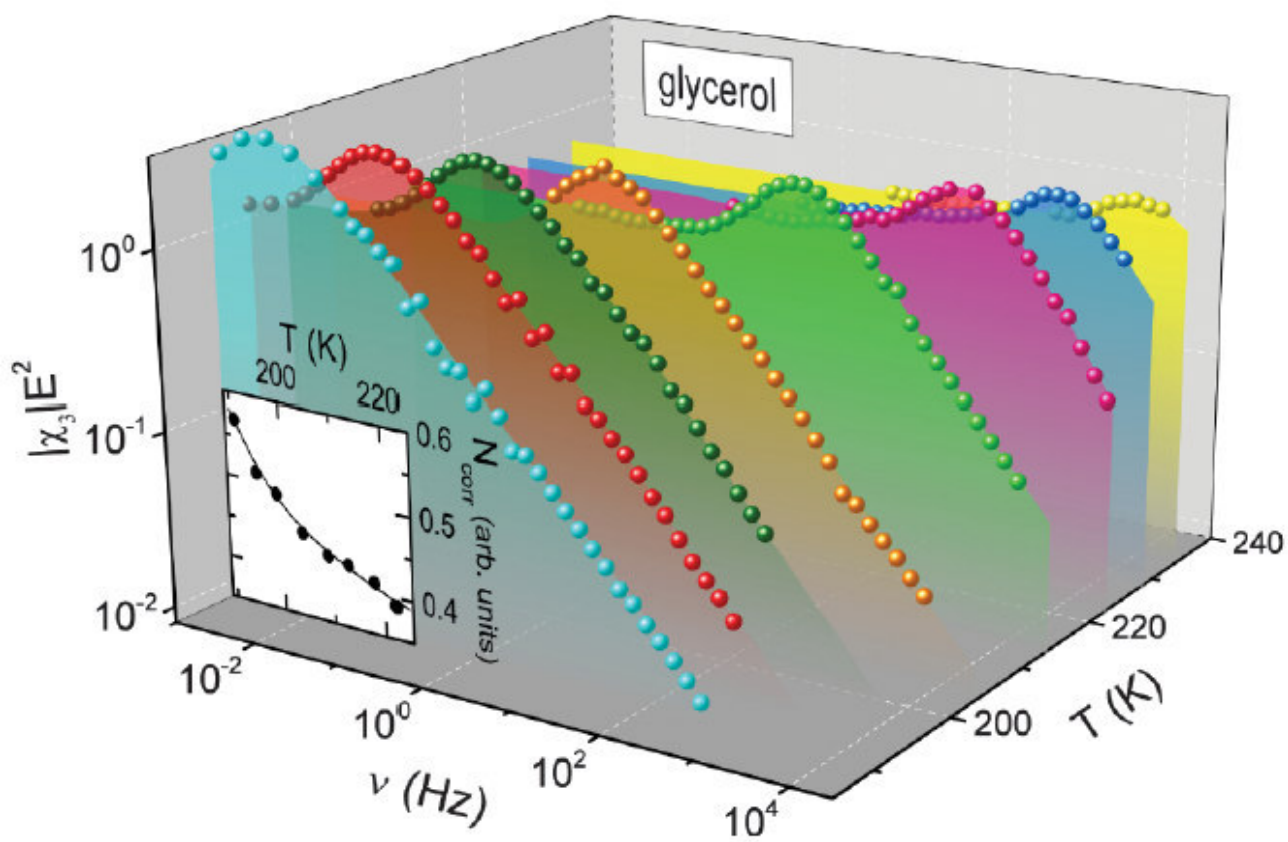

Abb. 2.16.: Spektrum für die dielektrische Suszeptibilität dritter Ordnung aufgetragen als $\left|\chi_{3} E^{2}\right|$ für verschiedene Temperaturen. Das Inset zeigt die Amplitude des Maximums von $\chi_{3}$, welches proportional zu $N_{\text {corr }}$ ist [y]. 


\section{Experimentelle Methoden}

In diesem Teil der Arbeit werden die verwendeten experimentellen Techniken und deren physikalische Grundlagen erläutert.

\subsection{Polymerproben}

Bei den in dieser Arbeit untersuchten Polymerproben handelt es sich einerseits um reine Polymethylmethacrylat-Proben (PMMA) und andererseits um Blockcopolymere $\mathrm{P}$ (MMA-co-CMA)- $b$-PBA in verschiedener Stöchiometrie.

Für die Untersuchung des nichlinearen Verhaltens wurde kommerziell hergestelltes PMMA verwendet. Einerseits wurde Plexiglas ${ }^{\circledR}$ von Evonik Industries verwendet. Dieses PMMA ist für technische Anwendungen hergestellt. Daher können in dem Material Zusätze wie beispielsweise UV-Absorber enthalten sein. Da jedoch trotz eventuell vorhandener Zusätze die $\alpha$-Relaxation sehr gut ausgeprägt ist und sich die gemessene Glasübergangstemperatur von der für reines PMMA erwarteten nicht signifikant unterscheidet, werden Effekte der Zusätze auf das Relaxationsverhalten der PMMA-Proben zunächst vernachlässigt.

Es wurden zum Vergleich jedoch zusätzlich Proben aus PMMA von Goodfellow gemessen, welches laut Hersteller frei von UV-Absorbern ist und sich durch eine hohe Reinheit auszeichnet. Die Proben lagen jeweils in Form von Platten mit einer Dicke von $2 \mathrm{~mm}$ (Evonik) beziehungsweise $1 \mathrm{~mm}$ (Goodfellow) vor. Diese Platten wurden in Probenstäbchen von einer Breite von ca. 0.7-1mm Breite und $20 \mathrm{~mm}$ Länge geschnitten.

Die im zweiten Teil der Arbeit untersuchten Blockcopolymere wurden von Katharina Tietz ${ }^{1}$ unter Verwendung der Reversiblen Additions-Fragmentierungs-Kettentransfer (RAFT)-Polymerisation hergestellt. Diese Methode gehört zu den kontrollierten freien radikalischen Polymerisationen. Diese Polymerisationen verbinden die Vorteile einer kontrollierten Polymerisation, welche Polymere mit einheitlichem Molgewicht und geringer Dispersität liefert, mit den Vorteilen einer freien radikalischen Polymerisation. Denn die freie radikalische Polymerisation ist besonders unempfindlich gegenüber Verunreinigungen.

${ }^{1}$ Arbeitsgruppe Vana, Institut für Makromolekulare Chemie der Georg-August-Universität Göttingen 


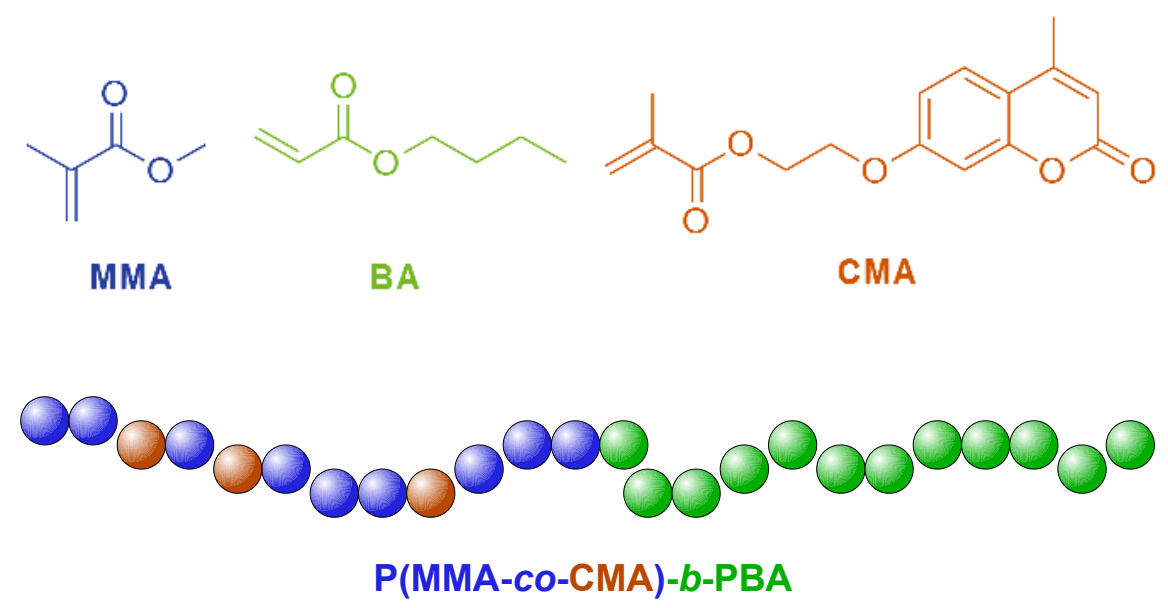

Abb. 3.1.: Schematischer Aufbau der Blockcopolymere

Die Blockcopolymere bestehen jeweils aus einem MMA-Block und einem BA-Block. Zusätzlich ist in den MMA-Block ein Coumarin-Derivat statistisch integriert. Die Abbildung 3.1 zeigt schematisch die einzelnen Konstituenten und den Aufbau der Blockcopolymere.

Die erhaltenen BCPs zeichnen sich aus durch typische Molgewichte im Bereich von (40000 - 110000) molg $^{-1}$ und Dispersitätsindizes von $1,2-1,6$. Die verschiedenen Proben unterscheiden sich in ihrem relativen Anteil des enthaltenen BA, sowie in der Gesamtkettenlänge, also dem Molekulargewicht. Der Coumarinanteil in den Proben liegt bei ca. $5 \%$.

\subsection{Thermogravimetrische Analyse}

Zur Untersuchung der thermischen Stabilität, wurden Proben mithilfe simultaner Thermogravimetrie und Dynamischer Differenzthermoanalyse untersucht. Für diese Messungen wurde ein STA 449C Jupiter der Firma Netzsch verwendet. Das Gerät bietet einen Temperaturbereich von Raumtemperatur bis $1773 \mathrm{~K}$ und weist die in Tabelle 3.1 gelisteten Spezifikationen auf. Das Gerät misst simultan die Masse, sowie ein DTA-Signal. Bei dem DTA-Signal handelt es sich um die Differenz der Temperaturen, die sich ergibt, wenn jeweils ein leerer Referenztiegel sowie der mit Probenmaterial gefüllten Tiegel mit gleicher Leistung geheizt werden. Aus diser Temperaturdifferenz kann anschließend $c_{p}$, die spezifische Wärme bei konstantem Druck, des Probenmaterials bestimmt werden, was Aufschluss über exo- oder endotherme Prozesse gibt, die während der Messung ablaufen. Um das Signal der Probe von dem der Tiegel zu trennen, wird jeweils vor der Messung eine Basislinie gemessen, bei welcher der später verwendete Tiegel leer gemessen wird. Zusätzlich 
bietet der Aufbau die Möglichkeit, die Bestandteile des Gases in der Probenkammer während der Messung mithilfe eines Massenspektrometers zu analysieren. Für die Messungen wurde ca. 20mg Probenmaterial in einem Aluminiumoxidtiegel eingewogen und unter Argonatmosphäre mit einer Rate von $5 \mathrm{~K} / \mathrm{min}$ auf $773 \mathrm{~K}$ aufgeheizt.

\begin{tabular}{ll}
\hline Massenauflösung & $1 \mu \mathrm{g}$ \\
$c_{P^{-}}$Meßgenauigkeit & $\approx 5 \%$ \\
Signal/Rausch-Verhältnis & $15 \mu \mathrm{W}$ (temperaturabhängig) \\
Basislinienlinearität & $3 \mu \mathrm{V}$ \\
Temperaturgenauigkeit(TE TypS) & $<1 \mathrm{~K}$ \\
\hline
\end{tabular}

Tab. 3.1.: Spezifikation der STA 449C Jupiter der Firma Netzsch. 


\subsection{Dynamisch mechanische Messungen}

Zur Untersuchung der globalen mechanischen Eigenschaften makroskopischen Probenmaterials, werden dynamisch mechanische Messungen durchgeführt. In der vorliegenden Arbeit wurde dafür ein DMA8000 von Perkin Elmer verwendet. Dieses Gerät bietet verschiedene Messmodi und Messgeometrien, die je nach untersuchtem Material und Fragestellung verwendet werden, wie im Folgenden erläutert wird. Die Steuerung der Messungen erfolgt einerseits über die mitgelieferte Software "DMA Control"der Firma Perkin Elmer, andererseits wurde für stark zeitaufgelöste Experimente das Gerät mit einem externen Rechner, sowie einer Analog-Digital-Wandler Karte gesteuert und ausgelesen.

\subsubsection{Aufbau und Spezifikation}

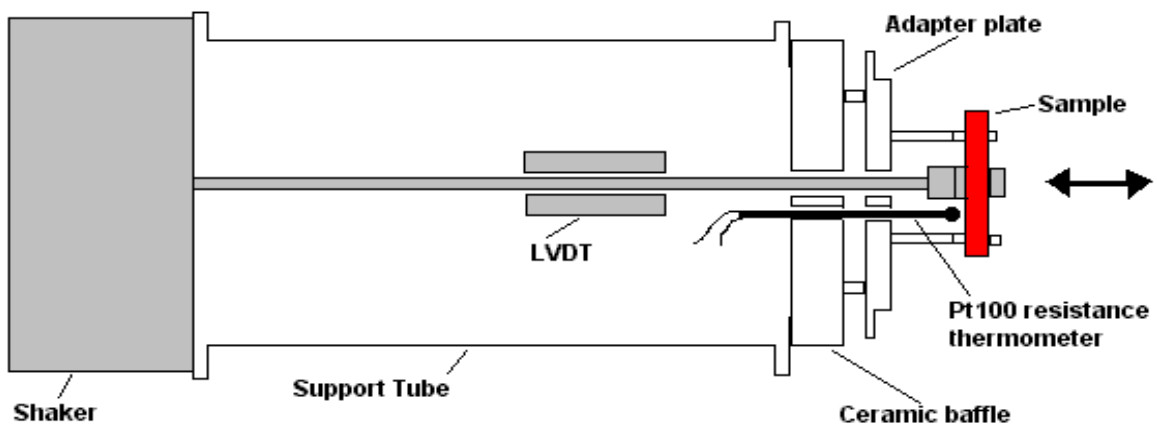

Abb. 3.2.: Schematischer Aufbau der DMA8000 (nach [107])

Ein dynamisch mechanischer Anaylsator untersucht die mechanischen Eigenschaften einer Probe, indem er diese einer oszillierenden mechanischen Spannung aussetzt und simultan die daraus resultierende Deformation der Probe aufzeichnet. Der schematische Aufbau des verwendeten DMA8000 ist in Abbildung 3.2 skizziert. Die Probe kann in verschiedenen Messgeometrien eingespannt werden, ist jedoch stets mit dem beweglichen Sondenstab verbunden. Dieser ist frei gelagert und an seinem hinteren Ende mit einem Kraftmotor verbunden, welcher aus einer Spule und einem Magneten besteht. Die Auslenkung des Sondenstabes wird mithilfe eines LVDT (Linear Variable Differential Transformator) gemessen. Dabei handelt es sich um ein abgeglichenes Spulenpaar, welches den Sondenstab umschließst und dessen Bewegung einen Spannungspuls im Spulensystem zur Folge hat. Für die Messungen können jeweils die Parameter Temperatur, dynamische Auslenkung, statische Kraft, sowie Frequenz individuell eingestellt werden. Die Spezifikationen die das Gerät laut Hersteller für diese Parameter bietet sind in Tabelle 3.2 zusammengefasst. Die Temperatur wird über ein Pt-Thermoelement erfasst. 


\begin{tabular}{lll} 
Parameter & Messbereich & Auflösung \\
\hline Frequenz & $0-300 \mathrm{~Hz}$ & $0,001 \mathrm{~Hz}$ \\
Auslenkung & $\pm 1000 \mu \mathrm{m}$ & $1 \mathrm{~nm}$ \\
Kraft & $\pm 10 \mathrm{~N}$ & $0,002 \mathrm{~N}$ \\
Temperatur & $83 \mathrm{~K}-673 \mathrm{~K}$ & abhängig von der Rate \\
\hline
\end{tabular}

Tab. 3.2.: Spezifikation der DMA8000 der Firma Perkin Elmer.

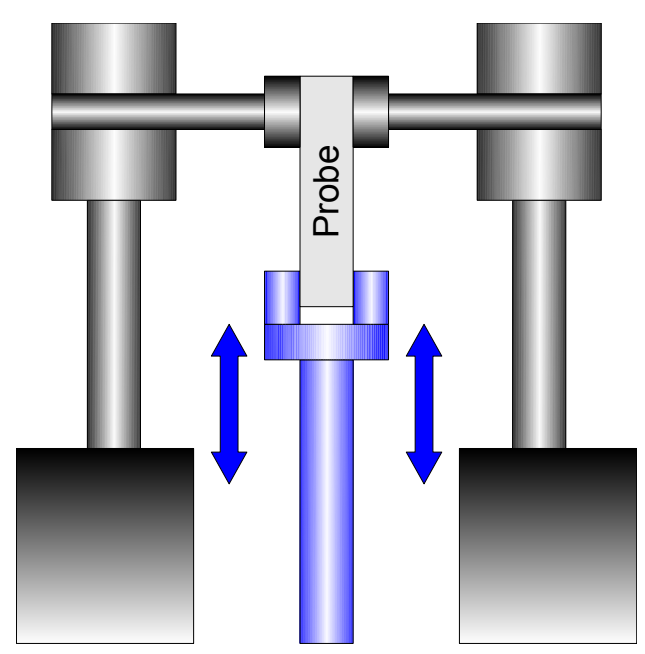

Abb. 3.3.: Messgeometrie im Zugversuch

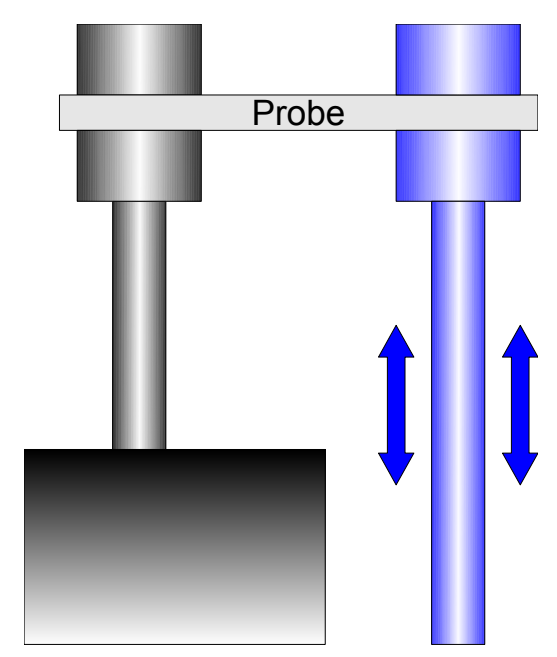

Abb. 3.4.: Messgeometrie im Biegebalkenexperiment

\subsubsection{Zugversuch}

Für Proben, die in mindestens einer Dimension sehr klein sind, wie Folien, Bänder oder Fasern ist diese Messgeometrie besonders geeignet. Die Probe wird erst in den beweglichen Probenstab eingespannt. Dabei sind einige wichtige Punkte zu beachten, damit Fehler bei der anschließenden Messung vermieden werden. Die Probe sollte möglichst senkrecht zwischen den Backen befestigt werden. Beim Festschrauben muss je im Wechsel eine der beiden Schrauben festgezogen werden, damit die bewegliche Backe nicht verkantet und somit die Probe rutschen kann. Ob es zu einer Verkantung gekommen ist wird überprüft, indem mithilfe einer Schieblehre die Breite beider Backen am oberen und unteren Ende verglichen wird. Dabei sollten sich die Werte nicht mehr als $0,1 \mathrm{~mm}$ unterscheiden. Werden sehr weiche Proben, wie Polymere in der Zuggeometrie gemessen ist es wichtig, dass die Schrauben keinesfalls zu fest angezogen werden, da dadurch der eingespannte Teil der Probe stark deformiert werden könnte. 


\section{Experimentelle Methoden}

\subsubsection{Biegebalkenexperiment}

Für stabförmige Proben hat sich ein Biegebalkenversuch bewährt. In diesem Experiment wird eine Seite der Probe fest eingespannt, während die andere Seite der Probe in dem beweglichen Probenstab eingespannt ist. Der Vorteil bei dieser Methode ist, dass keinerlei zusätzlichen statischen Kräfte notwendig sind und die Oszillation während der Messung um eine Nullinie stattfindet. Aus der Software der DMA 8000 können die Größen Spannung $\sigma$ und die Dehnung $\epsilon$ können aus den Rohdaten Kraft $F$ und Auslenkung $d$ über den Geometriefaktor $g_{f}$ und den strain-Faktor $s_{f}$ wie folgt berechnet werden [107],[102].

$$
\begin{aligned}
\sigma & =F \cdot \frac{s_{f}}{g_{f}}=F \cdot \frac{3 \cdot t}{l^{2}} \cdot\left(\frac{w \cdot(t / l)^{3}}{1+2.9(t / l)^{2}}\right)^{-1} \\
\epsilon & =d \cdot s_{f}=d \cdot \frac{3 \cdot t}{l^{2}}
\end{aligned}
$$

Die Größen $w, t$ und $l$ stehen für die Probendimensionen Breite, Dicke und Länge. Die Dehnnachbiebigkeit (englisch: Compliance) $C$ kann dann wie folgt bestimmt werden:

$$
C=\frac{\epsilon}{\sigma}=\frac{d}{F} \cdot g_{f}
$$

Ein weiterer Vorteil dieser Methode besteht in der Verwendung sogenannter Materialpockets für weiche oder pulverförmige Proben, die ohne Unterstützung nicht einzuspannen wären. Dafür wird das Probenmaterial in einen Umschlag aus rostfreiem Stahl gegeben, dieser wird zusammengefaltet und in die Apparatur eingebaut. Da Stahl einen konstanten Modul und einen konstanten Verlust in den untersuchten Temperaturbereichen aufweist, können alle Änderungen in den mechanischen Eigenschaften auf das Probenmaterial im Innern des Materialpockets zurückgeführt werden.

\subsubsection{Temperaturscans der Blockcopolymere}

Um das temperaturabhängige mechanische Verhalten der Blockcopolymere zu untersuchen, wurde das Probenmaterial mithilfe von Material Pockets in der Biegebalkengeometrie gemessen. Durch den Einsatz der Materialpockets können Proben der Blockcopolymere für verschiedene Zusammensetzungen mit der gleichen Methode gemessen werden, obwohl diese sich stark in ihren mechanischen Eigenschaften unterscheiden. Es liegen einerseits Butylacrylat-reiche Proben vor, welche nahezu flüssig sind, da $\operatorname{Tg}(\mathrm{BA})$ weit unterhalb Raumtemperatur liegt. Andererseits zeigen sich MMA-reiche Proben bei Raumtemperatur als ein sprödes Pulver. Die Messungen wurden mit einer Auslenkung von $0,02 \mathrm{~mm}$ und einer Frequenz von $5 \mathrm{~Hz}$ 


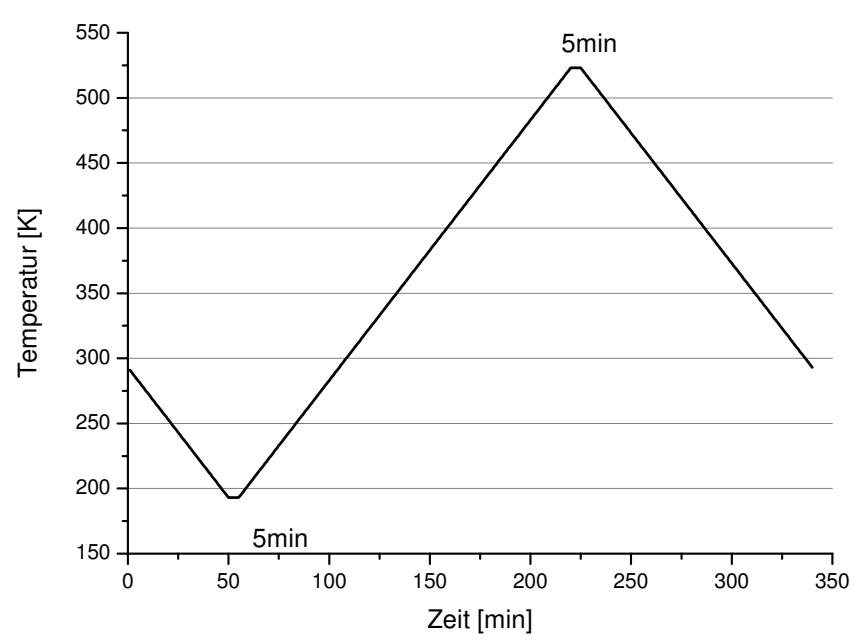

Abb. 3.5.: Temperaturprotokoll für die Charakterisierung der Blockcopolymere. Die Probe wird von Raumtemperatur mit einer Rate von $2 \mathrm{~K} /$ min auf $193 \mathrm{~K}$ gekühlt, nach 5 min Haltezeit wird das Material mit $2 \mathrm{~K} /$ min auf $523 \mathrm{~K}$ erhitzt, und anschließend auf Raumtemperatur gekühlt.

durchgeführt. Das dabei verwendete Temperaturprotokoll ist in Abbildung $\underline{3.5}$ dargestellt. Die Temperaturscans wurden hinsichtlich der Glastemperaturen wie folgt ausgewertet. Es wurde einerseits der Peak im $\tan \delta$ als Tg bestimmt. Dafür wurde ein Gaussfit an den Peak angepasst. Für einige der Proben war der Peak im $\tan \delta$ jedoch so schwach ausgeprägt, dass diese Art der Bestimmung nicht mehr möglich war. Alternativ konnte dann der Wendepunkt im Modul als $T_{g}$ bestimmt werden. Die Bestimmung des Wendepunktes kann auf zwei verschiedene Arten durchgeführt werden. Für glatte Kurven kann die Messkurve abgeleitet werden und das Maximum in der Ableitung mithilfe eines Gaussfits ermittelt werden. Ist die Messung jedoch verrauscht, beziehungsweise der Übergang sehr schwach ausgeprägt, so hat sich die sogenannte Tangentenmethode bewährt. Wie die Abbildung 3.6 zeigt, werden dabei jeweils 3 Tangenten an die Messkurve angelegt. Die Glasübergangstemperatur ist dann diejenige Temperatur, die genau zwischen den beiden Schnittpunkten der Tangenten liegt. Diese Methode ist jedoch sensitiv auf die Wahl der Bereiche, in dem die Tangenten gefittet werden.

Für die Blockcopolymere wurde jeweils der Glasübergang des Butylacrylates aus dem $\tan \delta$ und für den MMA-Anteil mittels der Tangentenmethode aus dem Modul bestimmt. Um eine gute Vergleichbarkeit der Temperaturen zu gewährleisten, wurden stets die Aufwärmkurven betrachtet und ausgewertet. Die Aufwärmkurven sind daher zu bevorzugen, da die Temperatur hierbei gleichmäßiger von dem Ofen des DMA gesteuert werden kann, als beim Abkühlvorgang. 


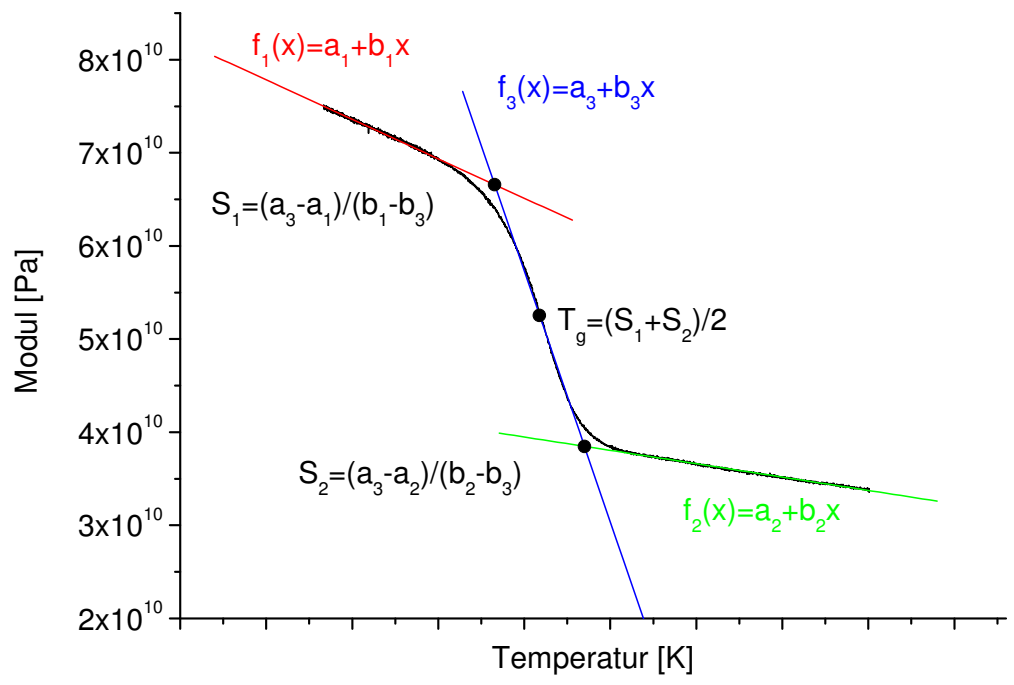

Abb. 3.6.: Bestimmung von Tg über den Wendepunkt im Modul mittels der Tangentenmethode, beispielhaft gezeigt an der Abkühlkurve der Probe 28(PMMA-co-Coumarin).

\subsubsection{Versuche mit UV-Bestrahlung}

Für die Bestrahlungsversuche wurde eine Quecksilberdampflampe XH 100 der Firma Müller $(100$ W) mit einem Lichtleiter LLEF der Firma Müller verwendet. Um die benötigten Wellenlängen herauszufiltern wurden zwei verschieden Filter eingesetzt. Für Wellenlängen von $\lambda>305 \mathrm{~nm}$ wurde ein Langpassfilter N-WG305 SCHOTT (ITOS) verwendet und ein Bandpassfilter F39-HG1 (AHF) liefert Licht der Wellenlänge 254nm. Die Blockcopolymerproben werden für die UV-Versuche im Zugversuch gemessen. Dafür werden rechteckige Bänder aus dem Probenmaterial herausgeschnitten. Die gemessenen Bänder haben typischerweise eine Breite von $2 \mathrm{~mm}$ bis $5 \mathrm{~mm}$ und eine Dicke von ca. 0,1 mm. Die Längen betragen zwischen $4 \mathrm{~mm}$ und $10 \mathrm{~mm}$. Zum Einspannen wird eine Vorspannung von $-0,2 \mathrm{~N}$ verwendet, damit die Proben im Zugversuch parallel zur Zugrichtung ausgerichtet sind. Vor Beginn der Bestrahlung wird für 5 min lang eine dynamische mechanische Messung mit einer Frequenz von $1 \mathrm{~Hz}$ und einer dynamischen Kraftamplitude von $0,02 \mathrm{~N}$ durchgeführt. Dann wurde die Probe für eine Stunde mit UV-Licht mit einer Wellenlänge $\lambda>310 \mathrm{~nm}$ bestrahlt und anschließend erneut für 5 min dynamisch gemessen. Dieser Ablauf wurde mehrfach wiederholt, so dass sich Änderungen im Modul in Abhängigkeit der Bestrahlungsdauer untersuchen lassen.

\subsubsection{LAOS-Messtechnik und Modifikation DMA8000}

Um das Auftreten nichtlinearen Verhaltens von PMMA-Proben zu untersuchen wurden diese in der Biegebalkengeometrie eingebaut und der folgende Messablauf ver- 
wendet. Die Probe wird bei einer bestimmten Temperatur und einer bestimmten statischen Spannung mit einer sinusförmigen Spannung angeregt. Abhängig von Temperatur, statischer und dynamischer Spannung, sowie Frequenz wird das Auftreten höherer Harmonischer in der Dehnungsantwort der Probe untersucht. Die Messungen wurden mit einem Dynamisch Mechanischen Analysegerät (DMA) von Perkin Elmer durchgeführt. Zusätzlich wurde ein Arbiträrfunktionengenerator an das Messsystem angeschlossen und eine Software programmiert, die individuelle Messabläufe zusätzlich zu den in der DMA8000 implementierten ermöglicht. Die Dehnungsantwort der Probe wird mittels eines 16 bit Analog-to-digital-converter (ADC) digitalisiert. Die verwendete ADC-card von National Instruments besitzt eine maximale Sampling-rate von $250 \mathrm{kHz}$ pro Kanal. Diese hohe Sampling-rate führt zu einer hohen zeitlichen Auflösung der Signale, was wichtig ist für die Frequenzanalyse mittels Fouriertransformation.

Zunächst wird ein Überblick über die experimentellen Bedingungen für das Auftreten des nichtlinearen Verhaltens gewonnen, indem die einzelnen Parameter : Frequenz, Temperatur und dynamische Kraftamplitude systematisch variiert werden. Es wurde dafür zunächst bei einer festen Temperatur die dynamische Kraftamplitude variiert und die jeweilige Dehnungsantwort des Materials mitgeschrieben. So konnte zunächst festgestellt werden, inwieweit die Methode geeignet ist nichtlineares Verhalten zu detektieren. Zudem kann für das jeweilige Material ein Überblick erhalten werden bei welchen äußeren Parameter Nichtlinearität auftritt, um anschließend weitergehende Messungen durchzuführen. Zu diesen Messungen gehört die Untersuchung der Zeitabhängigkeit. Diese Messmethode soll im Folgenden erläutert werden.

Um zu untersuchen, ob das Auftreten der Nichtlinearität mit einer bestimmten Zeitskala verknüpft ist, wurden zeitaufgelöste Messungen in Form eines NHN-Experiments (Niedrigfeld-Hochfeld-Niedrigfeld-Experiment) durchgeführt. Dafür wird die Probe erst mit einer dynamischen Kraft mit einer so kleinen Amplitude angeregt, dass die erwartete Materialantwort rein linear ist. Nach einer festen Anzahl Schwingungen wird die Kraftamplitude instantan auf einen so großen Wert erhöht, dass nichtlineares Verhalten zu erwarten ist. Anschließend wird die Amplitude wieder auf den kleinen Wert reduziert. Es wird sowohl die Anregung, also die Kraft $F(t)$ und die Antwort der Probe, die Auslenkung $D(t)$ für jede Periode fouriertransformiert und jeweils Amplitude $A$ und Phase $\phi$ der Signale bestimmt:

$$
\begin{gathered}
A=\sqrt{R e^{2}+I m^{2}} \\
\phi=\arctan (I m / R e)
\end{gathered}
$$




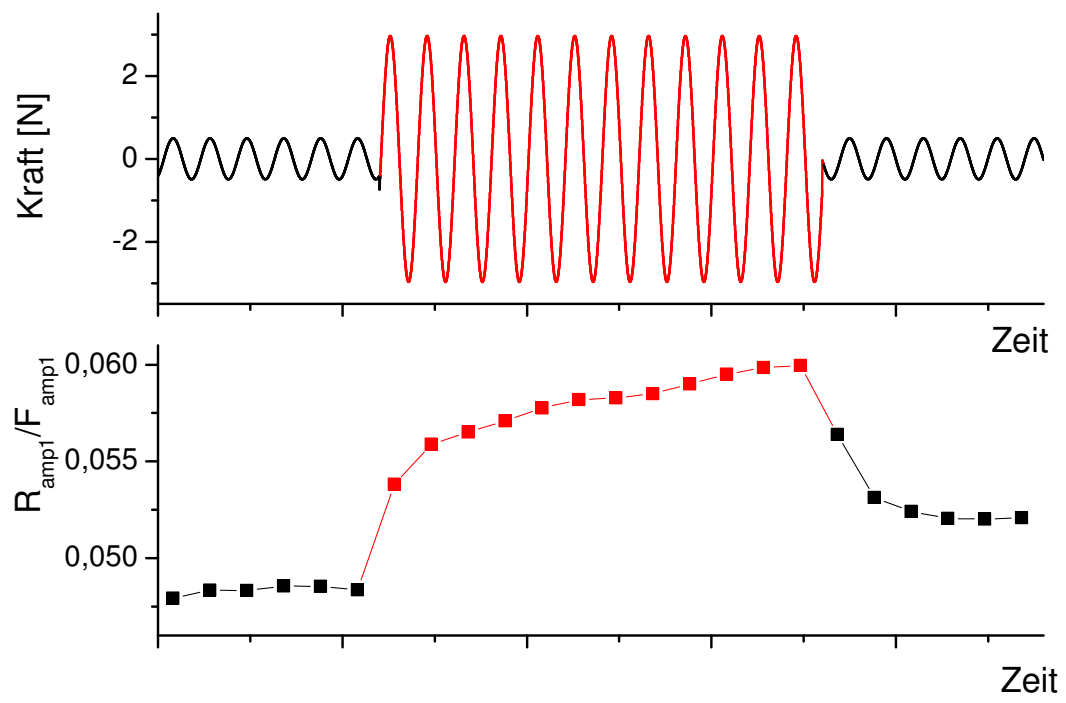

Abb. 3.7.: Typischer Zeitverlauf einer Messung. Der obere Graph zeigt die Kraftanregung und der untere zeigt eine typische Antwortfunktion, die für jede Periode fouriertransformiert wurde.

Dabei sind Im und Re jeweils der Imaginär- und Realteil, hier gezeigt für $\mathrm{F}(\mathrm{t})$ :

$$
\begin{aligned}
\operatorname{Re}(F(t)) & =\frac{\omega}{\pi} \int_{0}^{2 \pi / \omega} \sin (n \omega t) F(t) d t=A \cos (\phi) \\
\operatorname{Im}(F(t)) & =\frac{\omega}{\pi} \int_{0}^{2 \pi / \omega} \cos (n \omega t) F(t) d t=A \sin (\phi)
\end{aligned}
$$

Für $D(t)$ gelten die obigen Formeln entsprechend.

Die Abbildung 3.7 zeigt den Messablauf, sowie die Datenreduktion durch die periodenweise Fouriertransformation schematisch. Anhand der Abbildung wird deutlich, dass jeweils bei Änderung der Anregungsamplitude einen Phasensprung im Anregungssignal auftritt. Dies wird durch die Messsoftware hervorgerufen und sollte für nachfolgende Arbeiten durch eine alternative Software gelöst werden. So könnte das Antwortsignal dann direkt auf Phasenänderungen bei Erhöhung oder Reduzierung der Anregungsamplitude untersucht werden. Die periodenweise Fouriertransformation wurde mit einem Programm von Prof. Richert ${ }^{2}$ automatisch durchgeführt.

Während die sehr hohe dynamische Spannung auf die Probe wirkt tritt im Material vermehrt Dissipation auf. Dies kann dazu führen, dass sich das Probenmaterial deutlich erwärmt. Wird diese Wärme nicht schnell genug abgeführt, so akkumuliert sie sich und die Probe heizt sich immer weiter auf. Dieser Effekt kann in den

\footnotetext{
${ }^{2}$ Department of Chemistry and Biochemistry, Arizona State University, Tempe, Arizona 852871604, USA
} 
Experimenten beobachtet werden. Typische Temperaturverläufe während der Messungen sind im Anhang A zu finden. Anhand dieser Messdaten wird zudem deutlich, dass die Temperaturregelung im Bereich der Glasübergangstemperatur von PMMA $(T \approx 373 \mathrm{~K})$ nicht optimal ist, sondern die Temperatur vielmehr eine Schwingung mit einer Periode von 250s und einer Amplitude von etwa 0, $2 \mathrm{~K}$ zeigt. Da in dieser Arbeit jedoch zunächst das Experiment auf Konsistenz geprüft werden soll, sind diese Messungenauigkeiten erstmal weniger gravierend.

\subsection{Kleinwinkel-Röntgenstreuung}

Kleinwinkelstreuung ist eine besondere Form der experimentelle Röntgenbeugung, bei der die gestreute Strahlung unter sehr kleinen Winkeln $\left(2 \theta \leq 5^{\circ}\right)$ zum Primärstrahl auftritt. Gemäß der Bragg-Bedingung bedeutet ein kleinerer Streuwinkel, eine umso größere geordnete Struktur, an welcher die Strahlung gebeugt wurde. Mit dieser Methode können typischerweise Strukturen in der Größenordnung einiger Nanometer aufgelöst werden, so dass diese Technik vor allem für die Untersuchung von Kolloiden und Polymeren Anwendung findet.

Die untersuchten Blockcopolymere wurden vor dem Einbau auf eine Mylar-Folie aufgebracht. Der verwendete Aufbau besteht aus einer modularen Röntgenquelle der Firma iMOXS und arbeitet mit einer Kupferanode mit der Wellenlänge $\mathrm{Cu} \mathrm{K}_{\alpha}$ $\lambda=0.154 \mathrm{~nm}$ und typischen Energien von $8-9 \mathrm{keV}$. Der Röntgenstrahl wird mithilfe einer modernen Kratky-Kamera der Firma Xenocs, die unter anderem über Göbel-Spiegel verfügt, kollimiert [9]. Eine Kollimation des Strahles ist für die Kleinwinkelstreuung von großer Wichitgkeit, da eine zu hohe Divergenz der eingestrahlten Röntgenstrahlung dazu führen kann, dass unter den beobachteten Winkeln Anteile des Primärstrahles detektiert werden. Die gesamte Optik der Anlage befindet sich unter Vakuum, so dass keinerlei Streuung an Luftbestandteilen auftreten kann. Die gestreute Strahlung wird anschließend mithilfe eines Pixel-Detektors der Firma Pilatus detektiert. Die so erhaltenen zweidimensionalen Diffraktogramme wurden radialintegriert und die Intensität in Abhängigkeit des Wellenvektors $q$ dargestellt.Die Messungen wurden in Zusammenarbeit mit Oliva Saldanha ${ }_{-}^{3}$ durchgeführt.

\subsection{Rasterkraftmikroskopie}

Das Rasterkraftmikroskop, kurz AFM (Atomic force microscope) gehört zu den Rastersondenmikroskopen, und dient zur Oberflächenanalyse von Materialien im atomaren Bereich. Durch die Wahl geeigneter Sonden einerseits und passender Software und Berechnungsmodell können eine Vielzahl von Eigenschaften der Proben untersucht werden. Dadurch kann das AFM sehr vielseitig eingesetzt werden und gehört

\footnotetext{
${ }^{3}$ Arbeitsgruppe Köster, Institut für Röntgenphysik der Georg-August-Universität Göttingen
} 


\section{Experimentelle Methoden}

im Bereich der Nanotechnologie zu den Standard-Untersuchungsmethoden.

Das AFM arbeitet mithilfe einer Blattfeder, dem sogenannten Cantilever an dessen Ende sich die Messpitze befindet. Wird diese in die Nähe der Probenoberfläche gebracht, so kommt es zu einer Wechselwirkung mit den Atomen an der Oberfläche, so dass eine Kraft auf die Messpitze wirkt, die zur Verbiegung des Cantilevers führt. Die Abbildung 3.8 veranschaulicht dieses. Zur Detektion der Verbiegung ist ein Laserstrahl auf den Cantilever fokussiert, so dass sich bei Verbiegung der Winkel unter dem der Strahl reflektiert wird ändert. Dies wird mit einem positionssensitiven Photodetektor aufgezeichnet. Die Spitze wird kontrolliert in Bahnen über die Probe

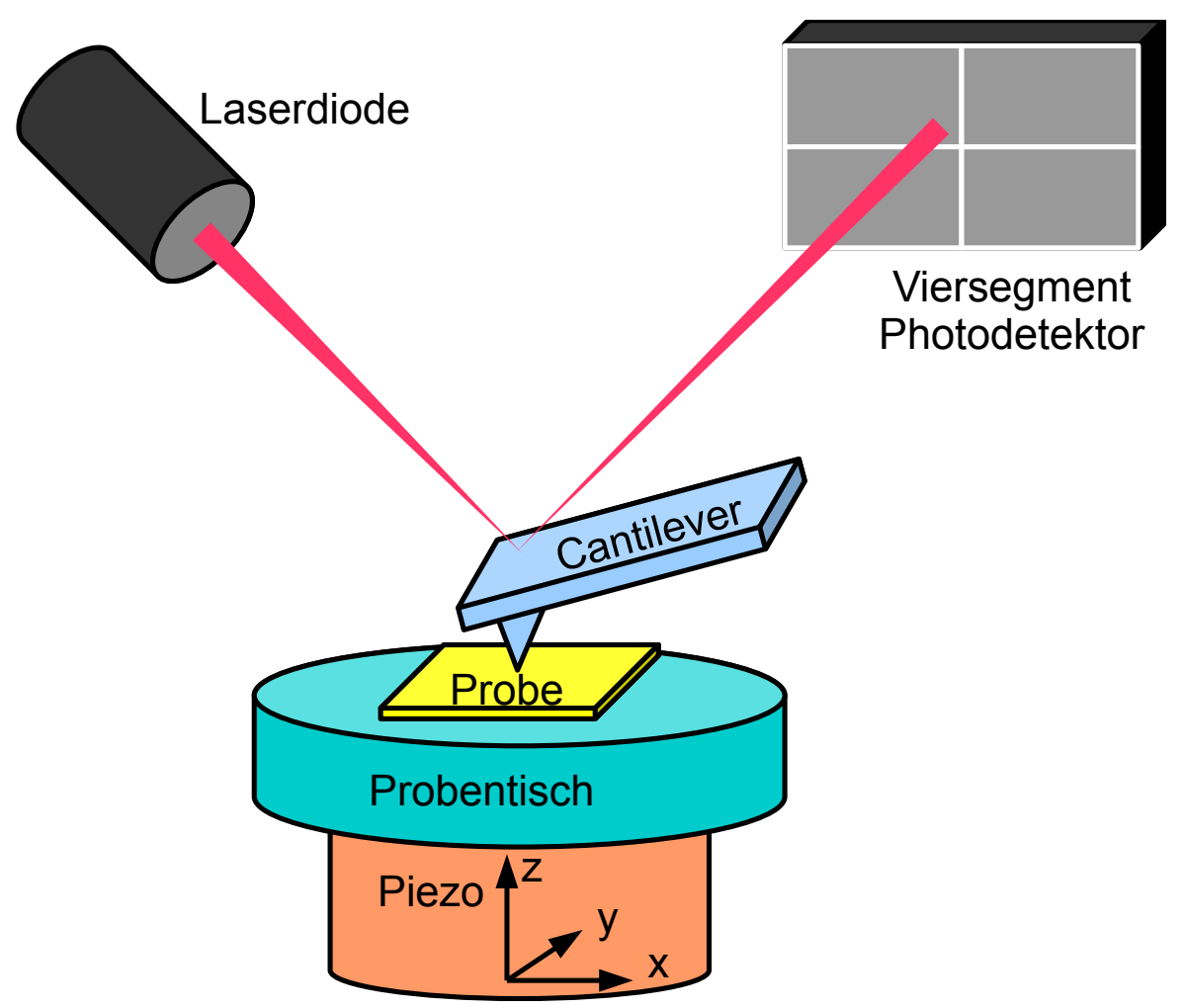

Abb. 3.8.: Funktionsprinzip des AFM. Schematisch dargestellt.

gefahren, und für jeden Punkt auf der Oberfläche die Cantileverbiegung aufgezeichnet. Dies ergibt schließlich ein zweidimensionales Bild der Probenoberfläche. Um die mechanischen Eigenschaften lokal zu untersuchen, können Kraft-Abstandskurven ausgewertet werden. Daraus lassen sich mithilfe geeigneter Modelliereung Modulus, Verlust, Viskosität und Adhäsion bestimmen [17].

Die AFM-Messungen wurden von Katharina Tietz ${ }^{4}$ an einem Multimode 8 AFM der Firma Veeco/Digital Instruments durchgeführt. Dass Gerät ist mit einem Nanoscope $\mathrm{V}$ Controller ausgestattet. Es wurde der PeakForce-QNM${ }^{T M}$-Modus für die Mes-

\footnotetext{
${ }^{4}$ Arbeitsgruppe Vana, Institut für Makromolekulare Chemie der Georg-August-Universität Göttingen
} 
sungen verwendet. Die verwendeten Cantilever hatten Federkonstanten von $3 \mathrm{~N} / \mathrm{m}$, $2 \mathrm{~N} / \mathrm{m}$ und $0,4 \mathrm{~N} / \mathrm{m}$. Es wurden Scanraten im Bereich von $0,5 \mathrm{~Hz}$ bis $1 \mathrm{~Hz}$ verwendet. 



\section{Nichtlineares Verhalten von PMMA}

Dieses Kapitel widmet sich der Untersuchung des nichtlinearen Verhaltens in dynamisch mechanischer Spektroskopie an Polymethylmethacrylat-Proben. Dafür wurden PMMA-Probenchargen zwei verschiedener Hersteller mit dem DMA8000 im Biegebalkenversuch untersucht. Zur besseren Übesicht wird im Folgenden das PMMA der Firma Evonik mit PMMA ${ }^{E}$ bezeichnet und das von Goodfellow mit PMMA ${ }^{G}$. Zur Charakterisierung der Proben, sowie für eine Einschätzung, bei welchen äußeren Parametern nichtlineares Verhalten zu erwarten ist, werden zunächst konventionelle Temperaturscans der Proben, sowie ein Spannungs-Dehnungs-Diagramm gezeigt. Anschließend wird das Auftreten des nichtlinearen Verhaltens qualitativ gezeigt. Dafür werden einerseits die Fourierspektren der Anregung und der Antwort der Proben untersucht, andererseits wird die Nichtlinearität und die Dissipation mittels Lissajousfiuguren visualisiert. Eine systematische Erhöhung der Spannungsamplitude für verschiedene Temperaturen wird anschließend gezeigt. Der zweite Teil dieses Abschnittes behandelt Messungen, bei denen die Fourierkomponenten zeitaufgelöst untersucht werden. Dabei werden einerseits für verschiedene Temperaturen unterhalb von $T_{g}$ und andererseits für verschiedene Spannungsamplituden, die Probenantwort bei der fundamentalen, sowie bei der 3. harmonischen Frequenz für beide PMMA gezeigt. Es werden daraus die Plateauwerte, sowie Zeitkonstanten bestimmt. Außerdem werden die Feldabhängigkeiten der nichtlinearen Dehnnachgiebigkeiten $C_{1}$ und $C_{3}$ gezeigt.

\subsection{Grundelegende mechanische Eigenschaften}

Das nichtlineare Verhalten in DMA-Experimenten wird in der Nähe von $T_{g}$ beobachtet. Es manifestiert sich im Spannungs-Dehnungs-Diagramm in Form eines Abweichens von dem streng linearen Verlauf, der für kleine Spannungen und kleine Temperaturen gefunden wird. Ein typisches Spannungs-Dehnungs-Diagramm ist in Abbildung $4.1 \mathrm{zu}$ sehen. Es wurde in der Biegebalkengeometrie für PMMA ${ }^{E}$ eine statische Kraft angelegt, die mit einer Rate von $0,5 \mathrm{~N} / \mathrm{min}$ auf $10 \mathrm{~N}$ (entspricht $\approx 15,2 \mathrm{MPa}$ ) erhöht wurde und dabei die resultierende Auslenkung der Probe aufgezeichnet. Es ist deutlich ein Abweichen vom linearen Verlauf für die Messungen bei $353 \mathrm{~K}$ und $363 \mathrm{~K}$ zu beobachten. Auch bei kleinen Spannungen verhält sich die 
Probe für diese Temperaturen nicht linear. Im Gegensatz dazu wird bei $298 \mathrm{~K}$ für kleine Spannungen ein linearer Verlauf gemessen, der jedoch bei höheren Spannungen auch ein leichtes Abweichen vom linearen Verhalten zeigt. Insgesamt kann beobachtet werden, dass das $\mathrm{PMMA}^{E}$ für größere Spannungen eine erhöhte Nachgiebigkeit zeigt.

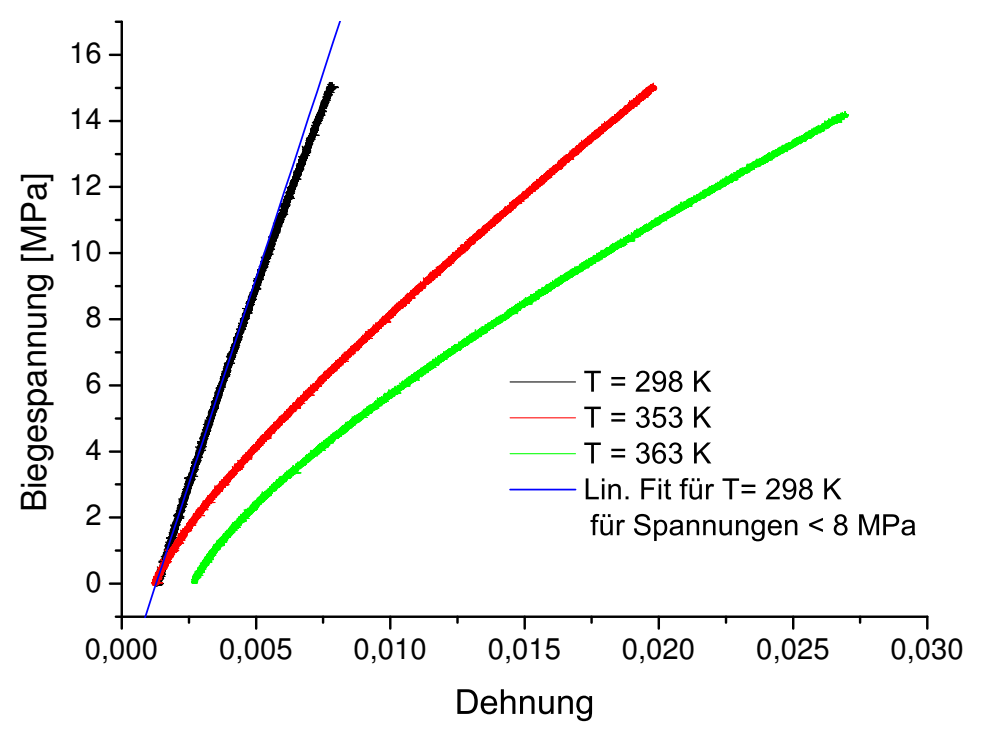

Abb. 4.1.: Spannungs-Dehnungsdiagramm für PMMA ${ }^{E}$ gemessen in Biegebalkengeometrie bei verschiedenen Temperaturen. Die Kraft wurde mit einer Rate von 0,5N/min bis auf einen Maximalwert von $10 \mathrm{~N}$ erhöht.

Die temperaturabhängigen mechanischen Eigenschaften beider Materialen im linearen Bereich wurden mithilfe von Temperaturscans mit dem DMA8000 bestimmt. Dafür wurde jeweils ein PMMA-Stab im Biegeversuch mit einer Auslenkung von 0,02 $\mathrm{mm}$ und einer Frequenz von $1 \mathrm{~Hz}$ beansprucht und dabei mit einer Rate von $2 \mathrm{~K} / \mathrm{min}$ auf $453 \mathrm{~K}$ geheizt. Die gemessenen Werte für den Speichermodul und den $\tan \delta$ sind in der Abbildung $\underline{4.2}$ dargestellt.

Wie erwartet, wird für beide PMMA-Proben ein sehr ähnlicher Verlauf beobachtet. Der Speichermodul liegt bei Raumtemperatur jeweils bei einem Wert von $2500 \mathrm{MPa}$ und zeigt eine deutliche Abnahme im Bereich der Glastemperatur. Diese wurde aus den Daten für den Modul auf einen Wert von $T_{g}\left(P M M A^{E}\right)=383 \mathrm{~K}$ und $T_{g}\left(P M M A^{G}\right)=390 \mathrm{~K}$ bestimmt. Der Peak im tan $\delta$ ist ebenfalls für beide Proben sehr deutlich ausgeprägt. Jedoch zeigt der Peak für das $\mathrm{PMMA}^{E}$ einen früheren Onset bei etwa $360 \mathrm{~K}$ und ist weniger scharf. Die Asymmetrie der Peaks ist auf den $\beta$-Prozess zurückzuführen. Dieser ist eindeutig für das $\mathrm{PMMA}^{E}$ stärker ausgeprägt, da hier die Asymmetrie stärker ist. Der Verlauf des $\tan \delta$ zeigt für das $\mathrm{PMMA}^{G}$ einen sehr scharfen Peak, der jedoch nach dem Maximum einen verrauschten Verlauf zeigt. Dies kann darauf zurückgeführt werden, dass aufgrund des Erweichens des Materials 


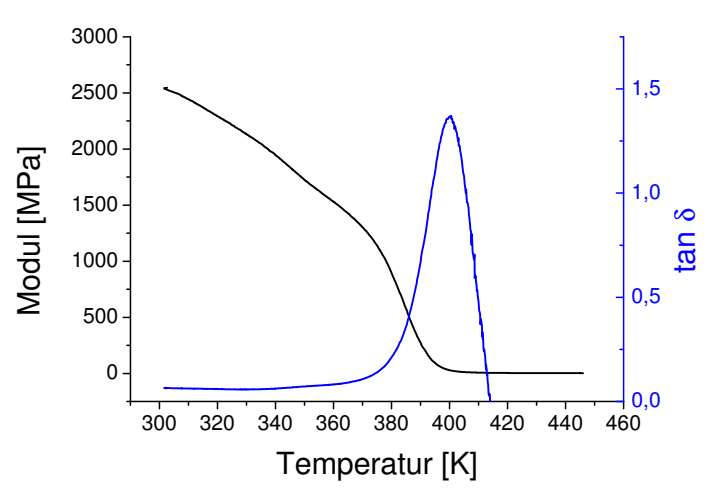

(a) PMMA $^{E}$

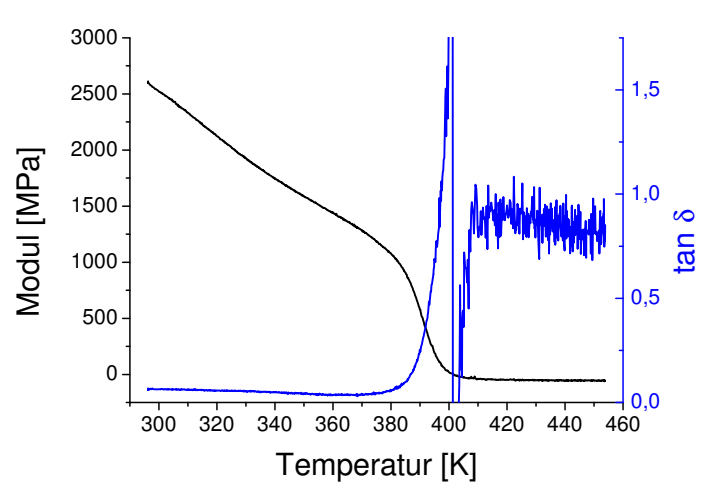

(b) PMMA $^{G}$

Abb. 4.2.: Temperaturabhängigkeit des Speichermoduls und des $\tan \delta$ einer PMMA ${ }^{E}$-Probe und einer PMMAG-Probe im Biegebalkenexperiment.

bei $T_{g}$, die Geometrie des Probenkörpers ändert. Daher wird sich in den folgenden experimentellen Ergebnissen auf Messungen unterhalb von $T_{g}$ beschränkt. Außer der Charaketistik am Glasübergang zeigen beide PMMA-Systeme keine weiteren Effekte im abgebildeten Temperaturbereich. Daher kann angenommen werden, dass die nachfolgend beobachteten Effekte nur aus der Physik des Glasüberganges stammen.

\subsection{Nichtlinearität in dynamisch mechanischen Messungen}

Wie sich das in dem Spannungs-Dehnungs-Diagramm beobachtete nichtlineare Verhalten in dynamisch mechanischen Messungen manifestiert, soll hier untersucht werden. Dafür wurde $\mathrm{PMMA}^{E}$ mit dem DMA bei einer Temperatur von $353 \mathrm{~K}$ bei verschiedenen dynamischen Spannungsamplituden und einer Anregungsfrequenz von $17 \mathrm{~Hz}$ gemessen. Die resultierende Dehnungsantworten sind in der Form von Lissajousfiguren in der Abbildung $\underline{4.3}$ dargestellt.

In Lissajousfiguren können Dissipation und Nichlinearität besonders anschaulich dargestellt werden. Die Lissajousfigur eines rein elastischen Verhaltens zeigt hierbei eine Linie. Im Gegensatz dazu wird ein rein viskoses Material einen Kreis in der Lissajous Darstellung zeigen. Für viskoelastische Materialien wird eine Ellipse beobachtet, deren umschlossene Fläche proportional zur dissipierten Energie ist. Für eine Spannung von $\sigma=2,2 \mathrm{MPa}$ reagiert das PMMA noch elastisch und das Lissjousdiagramm zeigt eine Linie. Für Spannungsamplituden von $\sigma=3,7 \mathrm{MPa}$ kommt es zu Verlusten im Material, was sich durch die elliptische Form zeigt. Für diese Anregung zeigt das Material folglich viskoelastisches Verhalten. Für größere Anregungsamplituden wächst die von der Kurve umschlosssene Fläche und damit die Dissipation. 

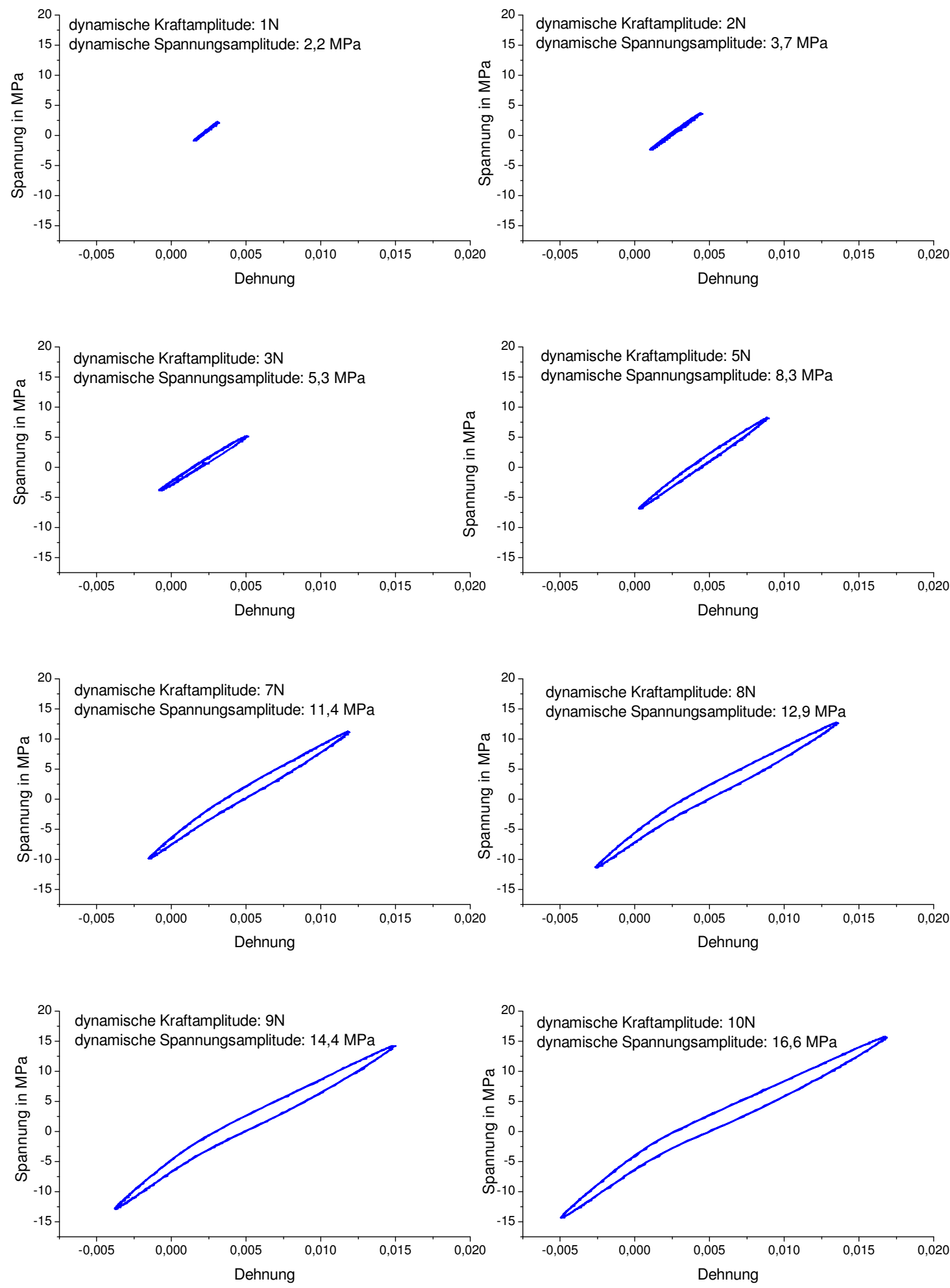

Abb. 4.3.: Lissajousfiguren für eine PMMA ${ }^{E}$-Probe im Biegebalkenversuch bei $353 \mathrm{~K}$ für verschiedene dynamische Kraftamplituden bei einer Temperatur von $353 \mathrm{~K}$. 
Für Amplituden größer als $\sigma=8,3 \mathrm{MPa}$ kann zudem eindeutig eine Abweichung von der elliptischen Symmetrie beobachtet werden. Dieser Symmetrieverlust ist auf den nichtlinearen Zusammenhang zwischen Anregungsspannung und Dehnungsantwort zurückzuführen.

Um die Abweichung vom linearen Verhalten zu quantifizieren werden die Signale fouriertransformiert und im Hinblick auf höhere Harmonische ausgewertet. Zunächst soll überprüft werden, ob schon das Anregungssignal höhere Harmonische enthält und inwieweit das Antwortsignal die erwarteten höheren Freqeunzen enthält. Dafür wurde eine PMMA ${ }^{E}$-Probe bei einer Frequenz von $17 \mathrm{~Hz}$ und einer Temperatur von $373 \mathrm{~K}$ mit einer dynamischen Spannungsamplitude von $10 \mathrm{MPa}$ angeregt und die Auslenkung der Probe mit hoher Zeitauflösung aufgezeichnet. Die Ergebnisse dieser Messung sind in der Abbildung 4.4 dargestellt. Auf der linken Seite sind das Signal der Anregungsspannung und der Dehnungsantwort dargestellt. Auf der rechten Seite befinden sich jeweils die Fourierspektren der Signale.
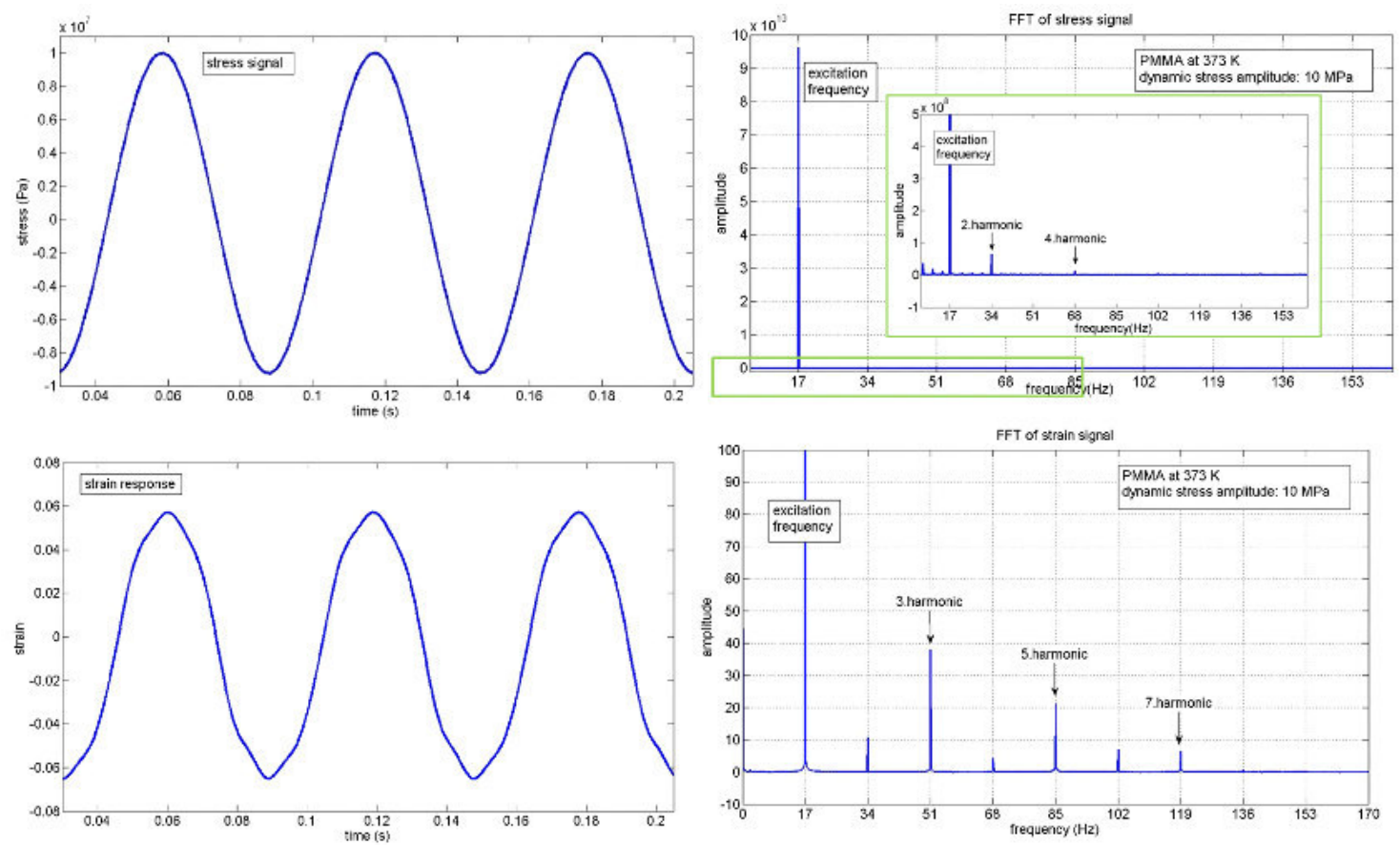

Abb. 4.4.: Spannungssignal und Dehnungssantwort (linke Seite) und die daraus bestimmten Fourierspektren für PMMA ${ }^{E}$ bei $T=373 \mathrm{~K}$.

Das Anregungssignal zeigt keine höheren harmonischen Beiträge bei den ungeraden Vielfachen der Anregung. Lediglich die zweite und die vierte Harmonische zeigen kleine Peaks im Fourierspektrum. Dabei beträgt jedoch schon die Amplitude bei $2 \omega$ weniger als $0,1 \%$ der Amplitude der Fundamentalen. Das Antwortsignal zeigt hingegen ein reicheres Fourierspektrum. Neben der Fundamentalen werden vor allem, wie erwartet, die ungeraden Harmonischen beobachtet. Die geraden Harmonischen werden auch detektiert, jedoch mit geringerer Intensität als die ungeraden. Die geraden 
Harmonischen können einerseits darauf zurückgeführt werden, dass schon das Anregungssignal minimale Anteile der geraden Harmonischen enthielt und anderereits werden sie erwartet, wenn das Experiment nicht ganz symmetrisch abläuft. Also wenn beispielsweise auch ohne äußere angelegte Kraft eine Spannung an der Probe wirkt. $\mathrm{Zu}$ einer solchen Spannung kann es durch denn Einbau der Probe kommen.

Um für weitere Analysen einen Überblick zu erhalten, welche Spannungen und Temperaturen geeignet sind, das nichtlineare Verhalten im PMMA zu untersuchen, wird das Amplitudenverhältnis $A_{3 \omega} / A_{1 \omega}$ als geeignete Größe für die Stärke der nichtlinearen Effekte, herangezogen. Dafür wurde eine $\mathrm{PMMA}^{E}$-Probe (mit einer Rate von $2 \mathrm{~K} / \mathrm{min}$ auf eine Temperatur unterhalb von $T_{g}$ geheizt und anschließend das folgende Messprotokoll durchgeführt. Die Probe wird mit $17 \mathrm{~Hz}$ und einer Kraftamplitude von $0,5 \mathrm{~N}$ dynamisch angeregt. Nach 10s wird die Probe mit einer höheren dynamischen Kraft angeregt und jeweils nach 1s Einschwingzeit, die Anregung und die Auslenkung der Probe aufgezeichnet. Die dynamische Amplitude wird jeweils um $0,1 \mathrm{~N}$ erhöht bis zu einem Wert von $8 \mathrm{~N}$. Für jede der Kräfte werden damit 170 Schwingungen aufgezeichnet, die anschließend einer Fouriertransformation unterzogen werden. Damit ergibt sich für jede Anregungsamplitde ein Fourierspektrum, aus welchem jeweils die Amplitude bei der dritten Harmonischen $\left(A_{3 \omega}\right)$ auf die entsprechende fundamentale Amplitude $\left(A_{1 \omega}\right)$ normiert werden kann. Die Auswertung wurde mithilfe von Matlab gemacht. Das Skript befindet sich im Anhang A. Dieses Verhältnis $A_{3 \omega} / A_{1 \omega}$ ist in Abbildung 4.5 für verschiedene Temperaturen unterhalb

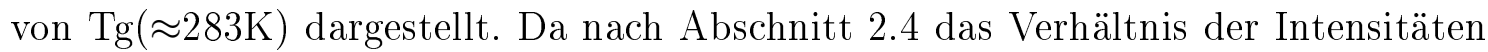
in erster Näherung proportional zum Quadrat der Anregungsamplitude sein muss, wurde es in der Abbildung gegen das Quadrat der Anregungsamplitude $\sigma_{0}^{2}$ aufgetragen. Diese Abbildung zeigt, dass $A_{3 \omega} / A_{1 \omega}$ für die Temperaturen zwischen $353 \mathrm{~K}$ und $368 \mathrm{~K}$ linear mit $\sigma_{0}^{2}$ wächst. Zu beachten ist jedoch, dass dieses Verhalten für den Bereich kleiner Spannungen noch nicht beobachtet werden kann. Dies kann jedoch auf die Auswertung zurückgeführt werden, denn für kleine Spannungen, ist das Signal zu Rauschverhältnis der 3. Harmonischen sehr schlecht, so dass im ersten Bereich die Werte weniger verlässlich sind. Für die Messung bei der höchsten Temperatur von $373 \mathrm{~K}$ kann kein eindeutiger linearer Zusammenhang beobachtet werden. Stattdessen ändert sich die Steigung immer wieder sprunghaft. Es wurde jeweils ein linearer Fit für die verschiedenen Kurven durchgeführt. Dabei wurde der Bereich kleiner Spannungen nicht berücksichtigt. Für die Messung bei der höchsten Temperatur wurde der Bereich gefittet, bevor die erste Steigungsänderung auftritt. Die so berechneten Steigungen sind in der Abbildung $\underline{4.6}$ in Abhängigkeit der Temperatur aufgetragen.

Die Temperaturabhängigkeit der Steigung $b$ kann mit einer exponentiellen Funktion angepasst werden. Aus dem Fit ergibt sich eine Konstante für das exponentielle Verhalten von $\approx \frac{1}{4}$. Dies zeigt sich auch am linearen Zusammenhang zwischen dem Logarithmus der Steigung $\left(\ln \left(b-b_{0}\right)\right)$ und der Temperatur,der in der Abbildung 4.7 gezeigt ist. Hervorzuheben ist, dass ein linearer Zusammenhang zwischen $\ln b$ und der Temperatur nicht gegeben ist, sondern der Offset $b_{0} \mathrm{zu}$ berücksichtigen ist. 


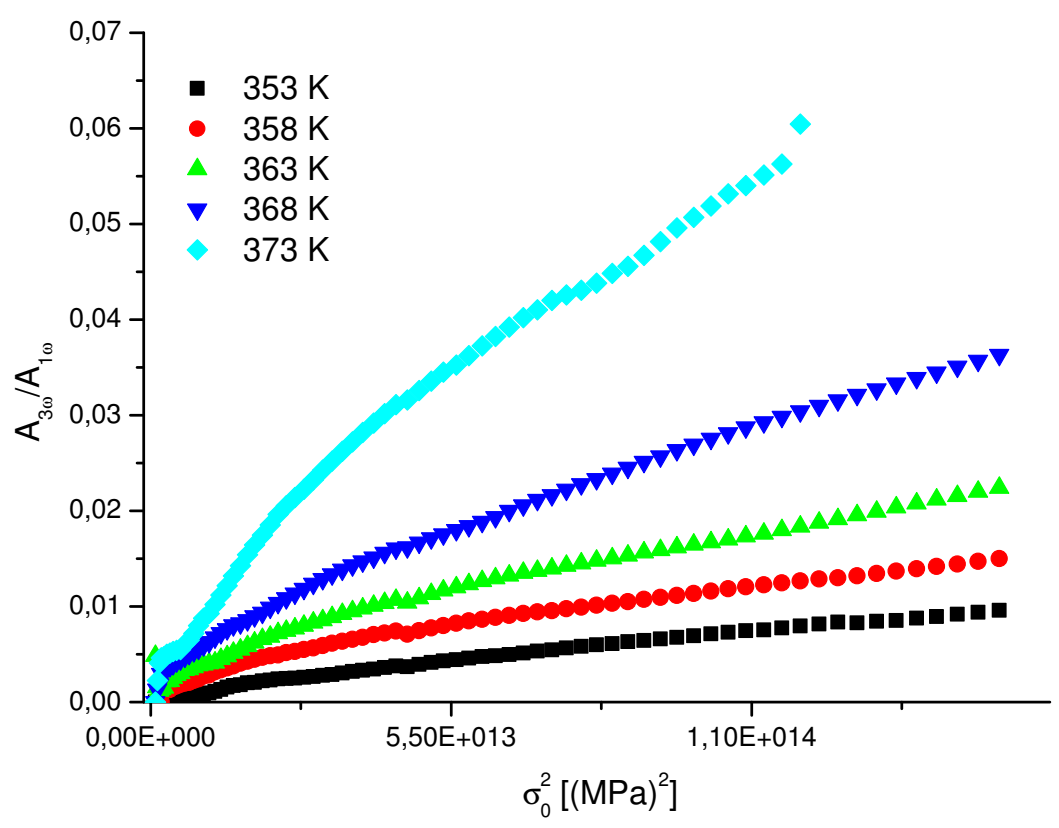

Abb. 4.5.: Verhältnis der Amplituden der Dehnungsantwort bei $\omega$ und $3 \omega$, aufgetragen gegen das Quadrat der Spannungsamplitude für verschiedene Temperaturen.

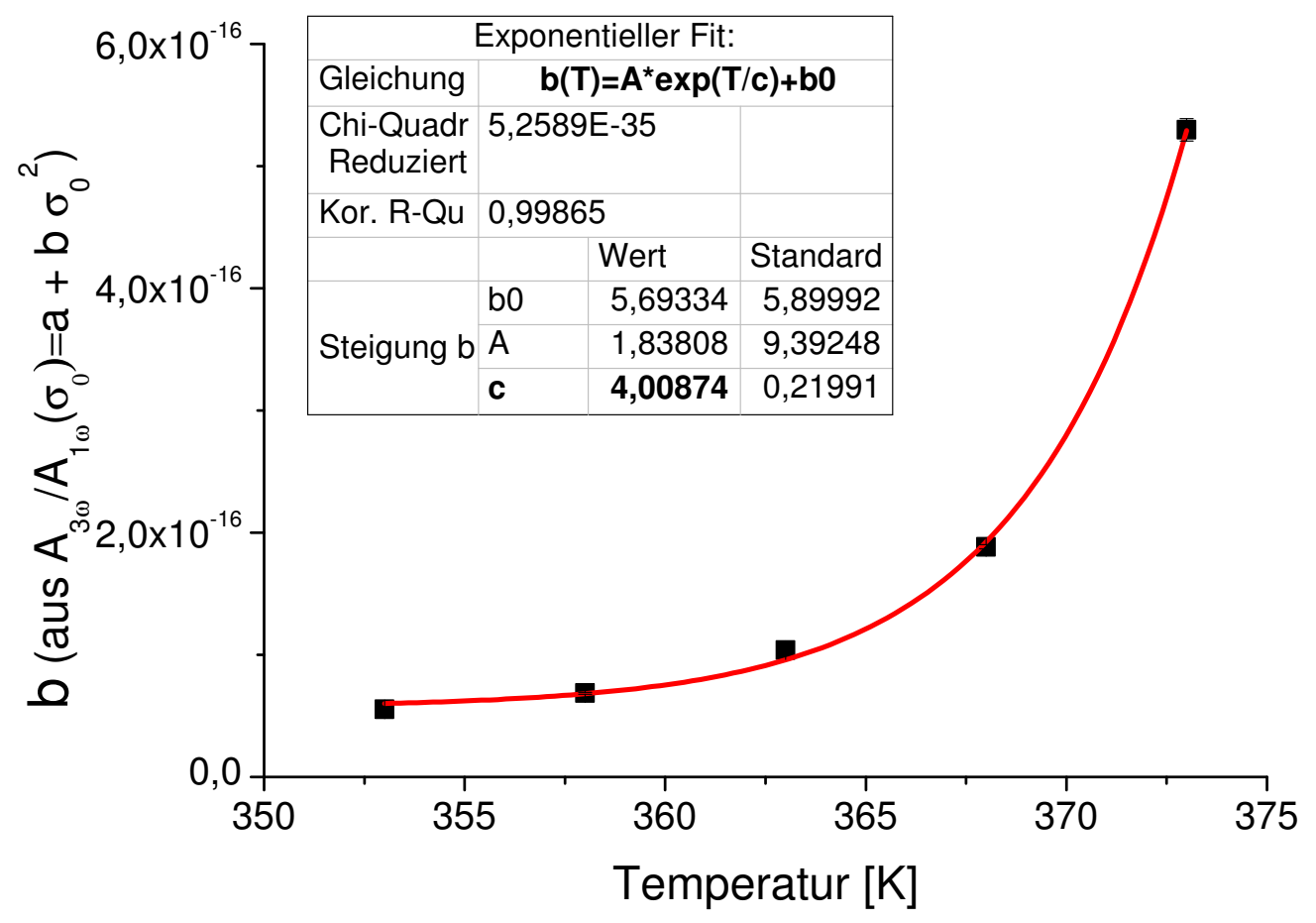

Abb. 4.6.: Steigung $b$ der Geraden $A_{3 \omega} / A_{1 \omega}\left(\sigma_{0}\right)=a+b \sigma_{0}^{2}$ 


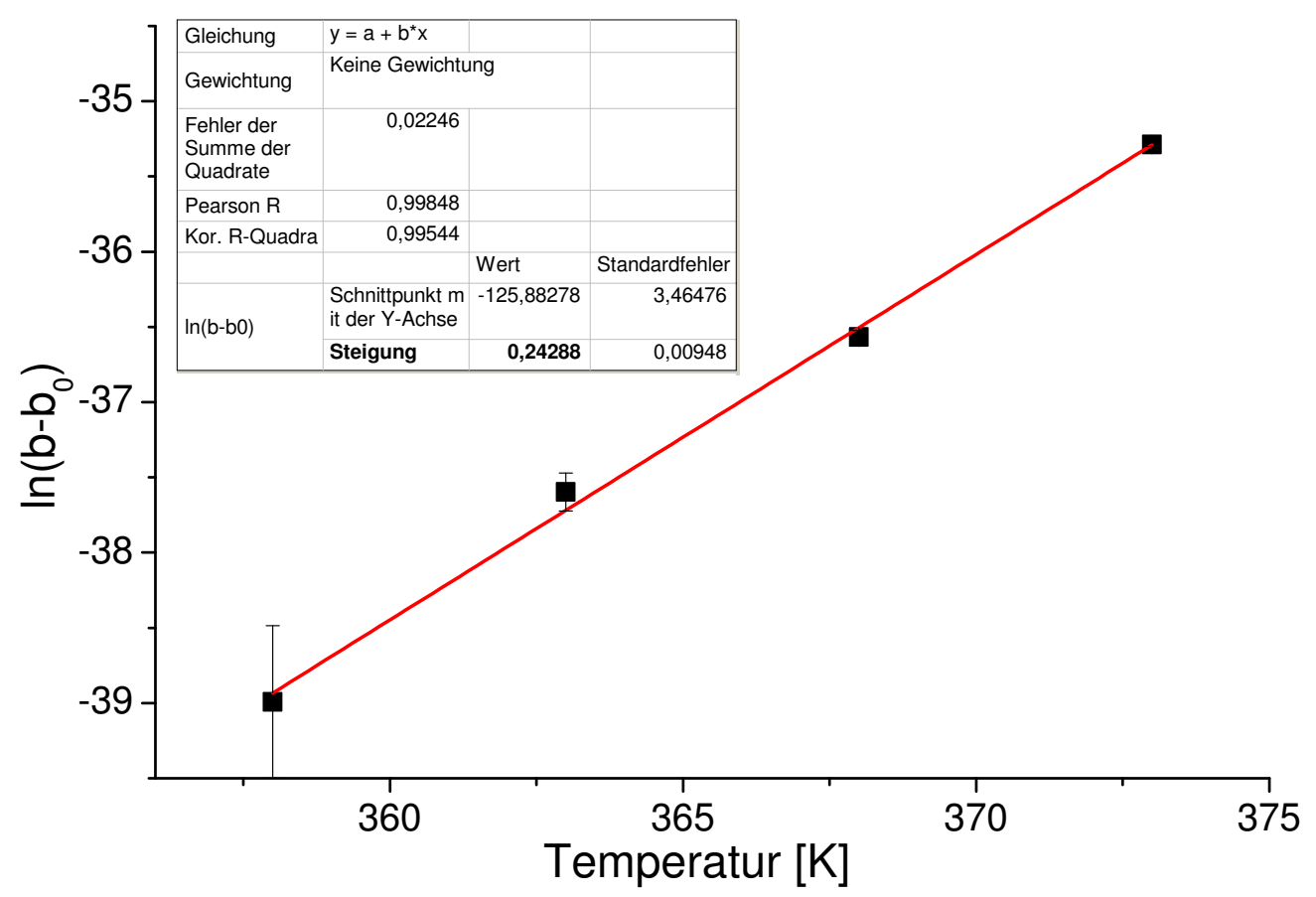

Abb. 4.7.: Die Auftragung von $\ln \left(b-b_{0}\right)$ in Abhängigkeit der Temperatur zeigt einen linearen Zusammenhang mit der Steigung 0,24.

Neben dem Anstieg der dritten Harmonischen zeigen mit steigender Spannungsamplitude auch weitere höhere Harmonische signifikante Beiträge. Dies ist in der Abbildung $\underline{4.8}$ dargestellt. Es sind hier jeweils die Amplituden für einige der ungeraden Harmonischen in Abhängigkeit der Spannungsamplitude aufgetragen. Es zeigt sich, dass ungefähr bei einer Spannung von $3 \mathrm{MPa}$ die Amnplituden der höheren Harmonischen stark ansteigen. Diese Spannung kann als ein kritische Grenze erfasst werden, ab welcher ein Depinning-Prozess stattfindet. Das bedeutet, das eine kritische Spannung erreicht ist, ab welcher großskaligere Bewegungen der Polymere möglich sind. 


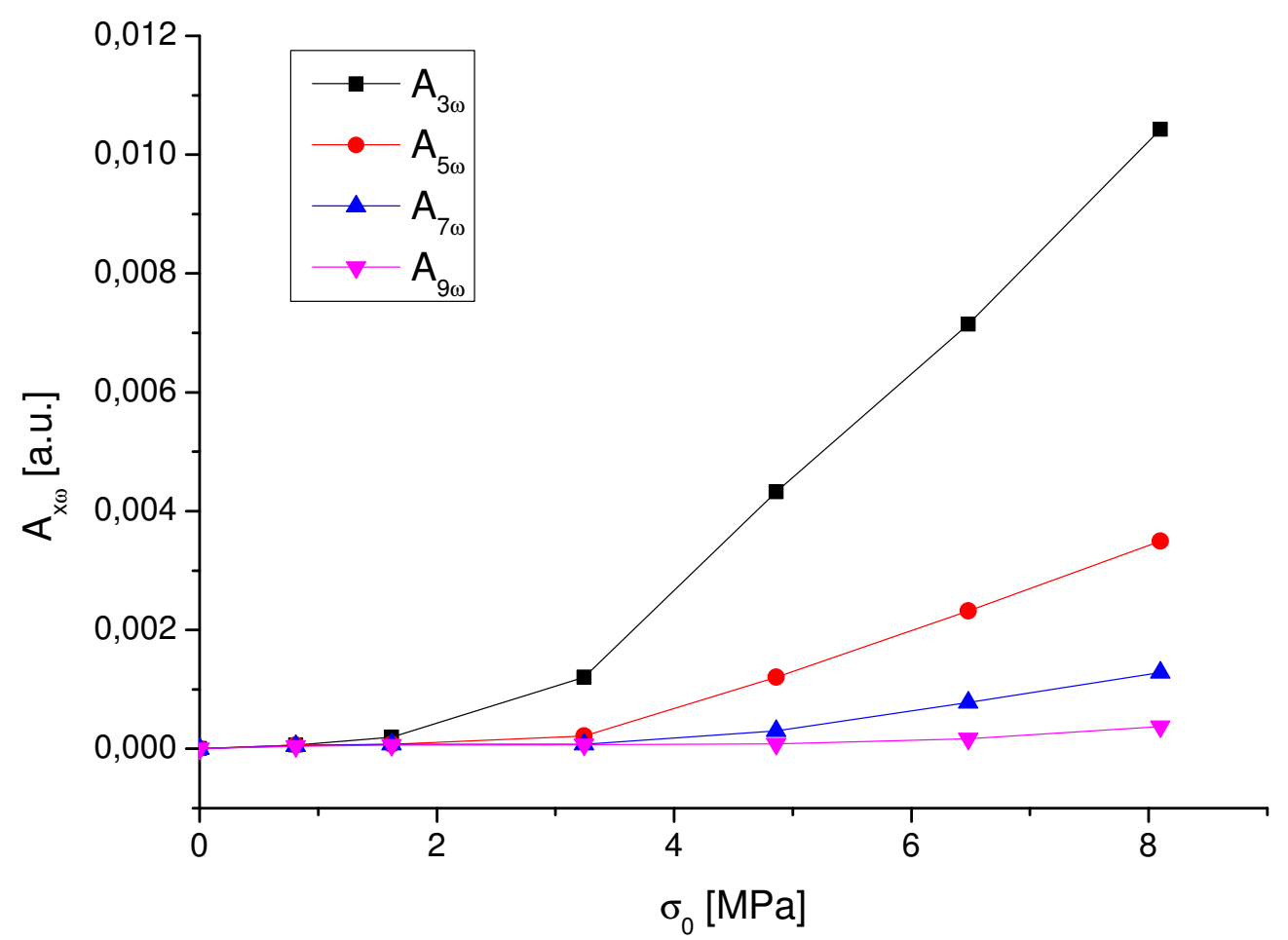

Abb. 4.8.: Amplituden der ungeraden Harmonischen der Probenantwort in Abhängigket der Spannungsamplitude für PMMA ${ }^{E}$ bei einer Temperatur von $T=373 \mathrm{~K}$.

\subsection{Zeitabhängigkeit der nichtlinearen Effekte}

Nachdem im vorangegangen Abschnitt zunächst Experimente vorgestellt wurden, die einen Überblick über das Auftreten der nichtlinearen Beiträge geben, soll hier zusätzlich noch die Zeitabhängigkeit der Nichtlienarität anaylsiert werden. Außerdem manifestiert sich, wie in der Theorie beschrieben, die Nichlinearität der Probenantwort nicht nur in der der Antwortamplitude bei der 3. Harmonischen, sondern auch in der Antwortfunktion bei der fundamentalen Frequenz. Experimentell können diese zusätzlichen nichtlinearen Beiträge zu $\epsilon_{1 \omega}$ über einen Vergleich der Nachgiebigkeit im Niedrigfeld mit der Nachgiebigkeit im Hochfeld ermittelt werden. Dafür wurde das in Abschnitt 3.3.6 vorgestellte NHN-Messprotokoll (Niedrigfeld-Hochfeld-Niedrigfeld) verwendet. Diese Messungen wurden einer periodenweisen Fouriertransformation unterzogen und zeigen daher zusätzlich die Zeitabhängigkeit der Nichtlinearität. Zunächst soll sich jedoch auf die Plateauwerte beschränkt werden und erst später soll die Zeitabhängigkeit untersucht werden. 


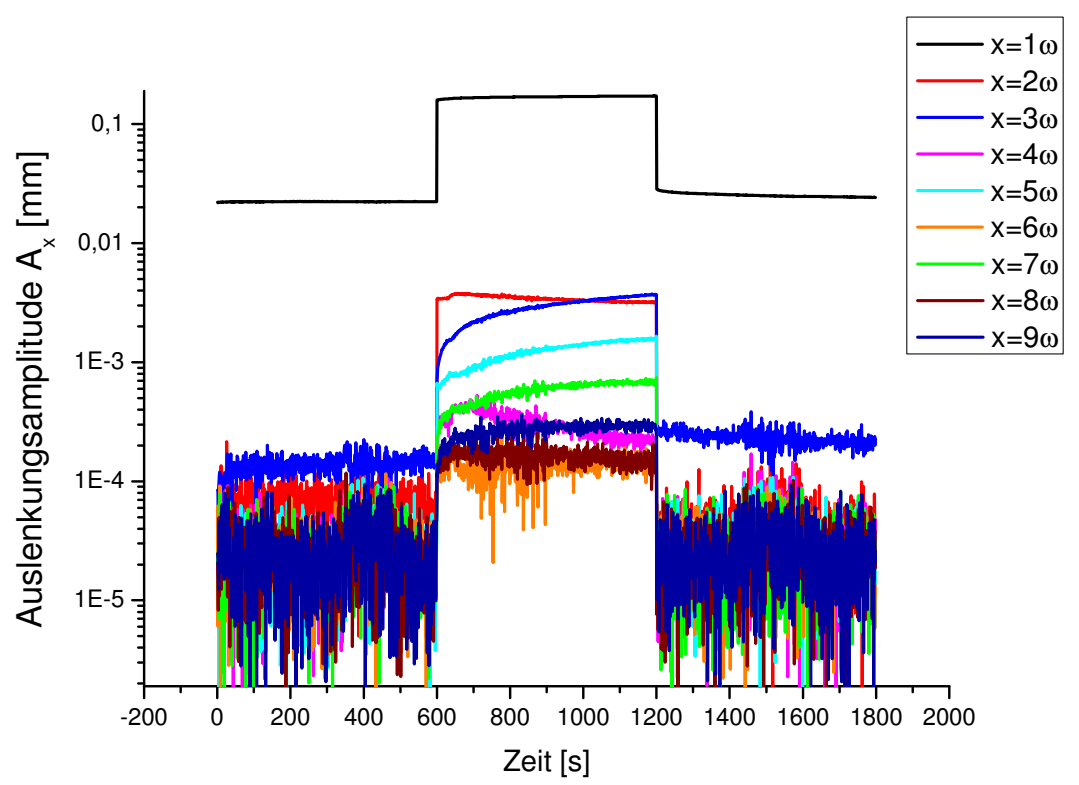

Abb. 4.9.: Fouriertransformierte Auslenkungsamplitude einer PMMA-Probe bei $373 \mathrm{~K}$ für verschiedene harmonische Frequenzen $i \omega$, der Anregungsfrequenz $\omega=1 \mathrm{~Hz}$. Die Kraftamplitude im niedrigen Feldbereich betrug $0,5 \mathrm{~N}(\approx 0,8 \mathrm{MPa})$ und im Hochfeldbereich $3 \mathrm{~N}$ $(\approx 5 \mathrm{MPa})$.

\subsubsection{Nichtlineares zeitabhängiges Verhalten von $\mathrm{PMMA}^{E}$}

Die Abbildung 4.9 zeigt für eine PMMA ${ }^{E}$-Probe bei $T=373 \mathrm{~K}$ den zeitlichen Verlauf der Antwortamplitude für verschiedene Harmonische $i \omega$ der fundamentalen Frequenz $\omega$ für ein solches NHN-Experiment. Im ersten Zeitabschnitt bis 600s, wird das Material mit einer kleinen Kraftamplitude von $0,5 \mathrm{~N}(\approx 0,8 \mathrm{MPa})$ angeregt, so dass davon ausgegangen wird, dass das Material rein linear darauf antwortet. In diesem Bereich zeigt die Auslenkung in der Fundamentalen Werte der Größenordnung 0,022 mm. Dazu im Gegensatz liefern die höheren Harmonischen deutlich geringere Beiträge. Die 3. Harmonische zeigt den größten Beitrag mit einem Wert von ca. $1,5 \cdot 10^{-4}$, was 0,7\% des Wertes der Fundamentalen entspricht. Nach 600s wird die Kraftamplitude sprunghaft auf $3 \mathrm{~N}(\approx 5 \mathrm{MPa})$ erhöht, was eine signifikante Änderung des Frequenzspektrums der Antwortfunktion zur Folge hat. In diesem Hochfeldbereich sind höhere Hamonische bis hin zur 9. Harmonischen im Antwortsignal der Probe zu beobachten. Die Beiträge der höheren Harmonischen steigen dabei zunächst sprunghaft an und zeigen dann eine Art Kriechverhalten, welches im Folgenden näher untersucht werden soll.

Für verschiedene Temperaturen unterhalb von $T_{g}$ wurde für $\mathrm{PMMA}^{E}$ jeweils das NHN-Messprotokoll verwendet und die Dehnungsantwort des Systems periodenweise fouriertransformiert. Die Abbildung 4.10 zeigt die komplexe Dehnungsamplitude $\epsilon_{1 \omega}^{*}$ der PMMA ${ }^{E}$-Proben bei der fundamentalen Frequenz $1 \mathrm{~Hz}$. 


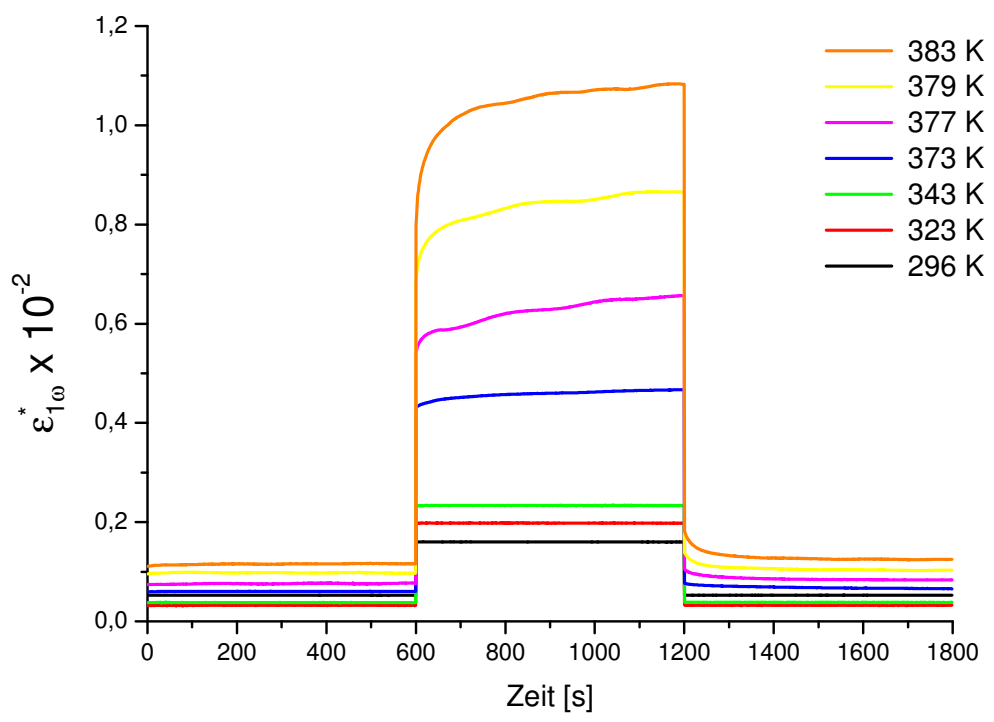

Abb. 4.10.: Erste harmonische Dehnungsamplitude $\epsilon_{1 \omega}^{*}$ für verschiedene Temperaturen für $P M M A^{E}$. Die Anregungsspannung im Niedrigfeldbereich betrug jeweils $\sigma_{0}=0,8 \mathrm{MPa}$ und im Hochfeldbereich $\sigma_{0}=5 \mathrm{MPa}$. Die verwendete Frequenz betrug $1 \mathrm{~Hz}$.

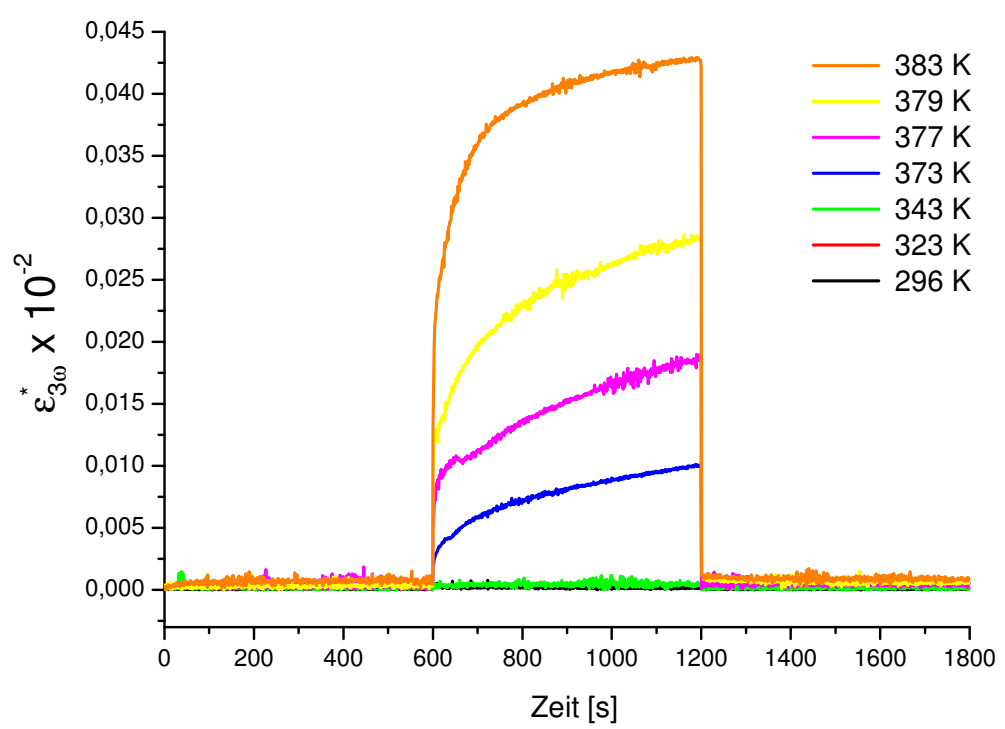

Abb. 4.11.: Komplexe Dehnungsamplitude $\epsilon_{3 \omega}^{*}$ für verschiedene Temperaturen für $P M M A^{E}$. Die Anregungsspannung im Niedrigfeldbereich betrug jeweils $\sigma_{0}=0,8 \mathrm{MPa}$ und im Hochfeldbereich $\sigma_{0}=5 \mathrm{MPa}$. Die verwendete Frequenz betrug $1 \mathrm{~Hz}$. 
Es kann eindeutig für alle Temperaturen ein instantaner Sprung auf einen höheren Amplitudenwert für den Hochfeldbereich beobachtet werden. Jedoch sind zwei Temperaturbereiche zu unterscheiden. Für die Temperaturen unterhalb $T=343 \mathrm{~K}$ zeigt sich keine Änderung des Hochfeldwertes mit der Zeit. Für die Messungen oberhalb von $343 \mathrm{~K}$ kann jedoch eine zeitliche Abhängigkeit beobachet werden. Es zeigt sich ein Kriechverhalten. Wird die Amplitude wieder auf den kleinen Anfangswert $\left(\sigma_{0}=0,8 \mathrm{MPa}\right)$ geregelt, so zeigt sich auch hier zunächst ein Sprung zu kleineren Werten gefolgt von einem Kriechprozess. Auffällig ist zudem, dass die Werte eine zusätzliche Schwingung zeigen. Dies ist auf die Temperaturregelung des DMA8000 zurückzuführen, wie im Abschnitt 3.3.6 beschrieben wurde.

Die Abbildung 4.11 zeigt die komplexe Amplitude der Dehnungsantwort bei der 3 . Harmonischen der Anregungsfrequenz $\epsilon_{3 \omega}^{*}$. Auch hier kann eindeutig eine Zeitabhängigkeit für Temperaturen oberhalb von $343 \mathrm{~K}$ für den Hochfeldbereich beobachtet werden. Dabei ist jedoch auffällig, dass hier am Ende der Hochfeldphase die Kurven noch eine eindeutige Steigung aufweisen. Das bedeutet, dass für die dritte harmonische Dehnung der Gleichgewichtszustand noch nicht erreicht ist. Dies ist ein Hinweis darauf, dass die Zeitkonstante für die dritte Harmonische größer ist, als für die Fundamentale. Für Temperaturen unterhalb von $343 \mathrm{~K}$ zeigt die dritte Harmonische keine Amplitude im Rahmen der Messauflösung. Im Bereich der kleinen Anregungsamplitude können auch keine signifikanten Beiträge der dritten Harmonischen beobachtet, so dass hier das für die Fundamentale beobachtete Kriechverhalten nicht $\mathrm{zu}$ beobachten ist.

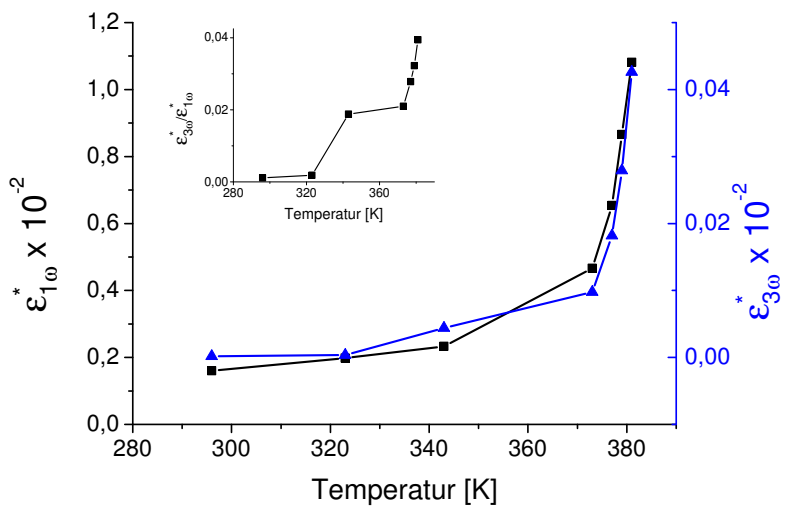

(a)

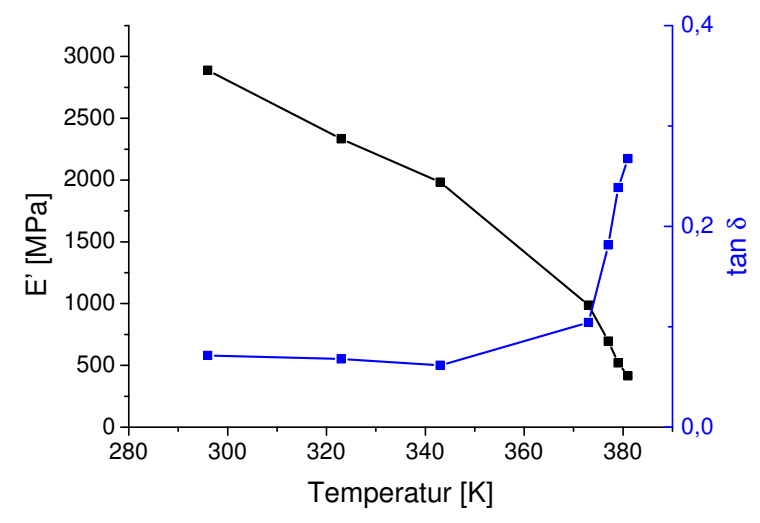

(b)

Abb. 4.12.: a)Plateauwerte für die komplexe Dehnungsamplituden bei der fundamentalen und der dritten Harmonischen der Anregungsfrequenz $\epsilon_{1 \omega}^{*}$ und $\epsilon_{3 \omega}^{*}$ für PMMA ${ }^{E}$. Das Inset zeigt die Temperaturabhängigkeit des Verhältnisses $\epsilon_{3 \omega}^{*} / \epsilon_{1 \omega}^{*}$ b) Speichermodul und Verlust bestimmt aus den Plateauwerten von $\epsilon_{1 \omega}^{*}$. Die gezeigten Werte wurden aus den letzten 100 Perioden des Hochfeldbereiches bestimmt. In diesem Bereich hatte die Anregung eine Spannungsamplitude von $5 \mathrm{MPa}$ bei einer Frequenz von $\omega=1 \mathrm{~Hz}$. 


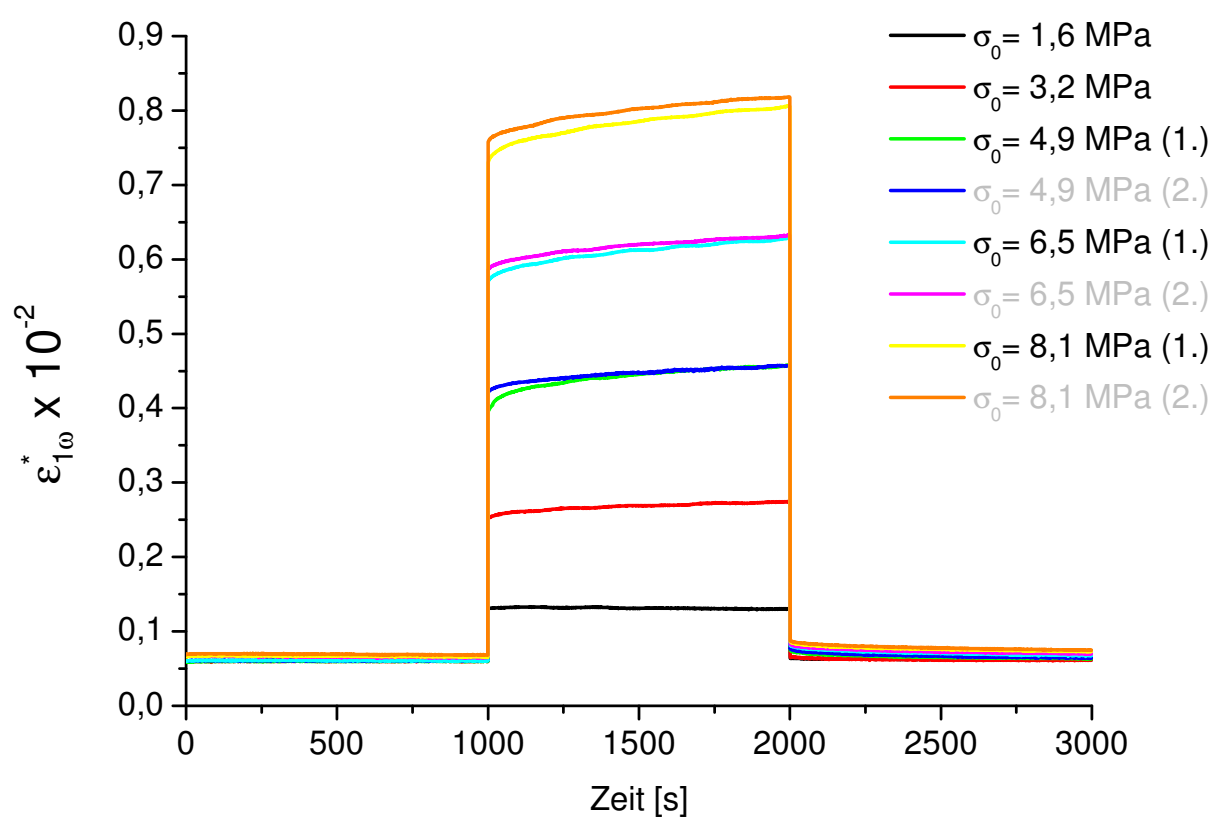

Abb. 4.13.: Dehnungsantwort bei der fundamentalen Frequenz $1 H z, \epsilon_{1 \omega}^{*}$ für verschiedene dynamische Kraftamplituden bei $T=373 \mathrm{~K}$ für $P M M A^{E}$.

Die Abbildung 4.12 zeigt die Plateauwerte für $\epsilon_{1 \omega}^{*}, \epsilon_{3 \omega}^{*}, \tan \delta$ und den Speichermodul $E^{\prime}$ für die verschiedenen Temperaturen. Da in dem beobachteten Zeitbereich keine Sättigung der Größen eingetreten ist, wurde als Plateauwert jeweils der Mittelwert der 100 letzen Perioden in der Hochfeldphase bestimmt. Es zeigt sich ein Anstieg sowohl der ersten harmonischen Dehnunsantwort $\epsilon_{1 \omega}^{*}$, als auch von der Dehnung bei $3 \omega, \epsilon_{3 \omega}^{*}$. Dabei wächst jedoch $\epsilon_{3 \omega}^{*}$ relativ zu $\epsilon_{1 \omega}^{*}$ stärker an, was durch das im Inset dargestellte Verhältnis der beiden Größen verdeutlicht wird. Für $E^{\prime}$ und tan $\delta$ ergibt sich das aus dem DMA-Temperaturscan bekannte Bild. Es werden bei Annäherung an $T_{g}$ ein Absinken des Speichermoduls und ein simultaner Anstieg des Verlustes $(\tan \delta)$ beobachtet.

Die Abhängigkeit der nichlinearen Beiträge von der externen Spannung wird im folgenden Abschnitt für PMMA ${ }^{E}$ gezeigt. Dafür wurden die PMMA-Proben wieder einem NHN-Messablauf unterzogen. Dabei wurden jeweils verschiedene Spannungsamplituden im Hochfeld $\sigma_{0}^{h}$ verwendet. Die Abbildung 4.13 zeigt die komplexe Dehnungsantwort bei der fundamentalen Frequenz $1 \mathrm{~Hz}$ und einer Temperatur von $T=373 \mathrm{~K}$ und in Abbildung $\underline{4.14}$ sind die dazugehörigen Signale der Dehnung bei der 3. harmonischen Frequenz dargestellt.

Sowohl die Dehnung bei der Fundamentalen, als auch bei der dritten Harmonischen zeigen das typische zeitabhängige Verhalten, für Spannungsamplituden $\sigma_{0} \geq$ 3, $2 \mathrm{MPa}$. Nach einem Sprung, sobald der Hochfeldbereich beginnt, ist jeweils ei- 


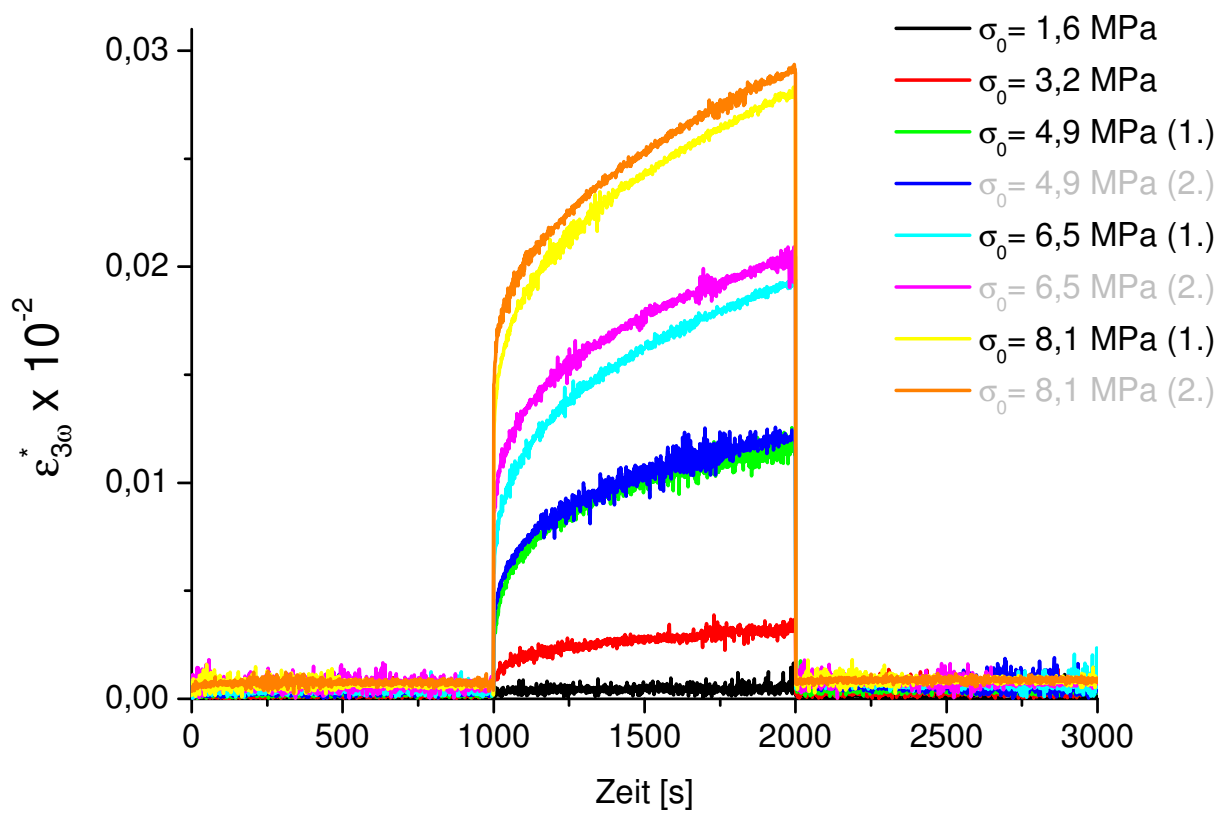

Abb. 4.14.: Antwort der Dehnung bei der 3. Harmonischen der Anregungsfrequenz $\epsilon_{3 \omega}^{*}$ für verschiedene dynamische Kraftamplituden bei $T=373 \mathrm{~K}$ für $P M M A^{E}$.

ne weitere zeitabhängige Zunahme der Dehnung zu beobachten. Dabei ist für $\epsilon_{3 \omega}^{*}$ der Sättigungswert am Ende des Hochfeldbereiches eindeutig nicht erreicht und die Kurve zeigt dort noch eine ausgeprägte Steigung. Im Gegensatz dazu hat $\epsilon_{1 \omega}^{*}$ am Ende der Hochfeldphase schon nahezu ein Plateau erreicht. Um trotz fehlender Sättigung Plateauwerte vergleichen zu können, wurden diese ebenfalls als Mittelwert über die 100 letzten Perioden im Hochfeld bestimmt. Diese Plateauwerte zeigen die in der Abbildung 4.15a dargestellte Abhängigkeit von der Spannungsamplitude $\sigma_{0}$. Dabei zeigt $\epsilon_{1 \omega}^{*}$ einen nahezu linearen Verlauf, wie es nach Gleichung 2.27 erwartet wird. Dazu im Gegensatz zeigt $\epsilon_{3 \omega^{*}}^{*}$ zwar auch einen Anstieg, jedoch wird die erwartete kubische Abhängigkeit von der Spannungsamplitude nicht beobachtet. Neben der Abbildung sind der aus den Plateauwerten bestimmte Speichermodul und der Verlust zum Vergleich dargestellt. Es zeigen sowohl der Speichermodul, als auch der Verlust einen Abfall mit steigender Spannung. Es wird jedoch ein Anstieg des Verlustes erwartet. 


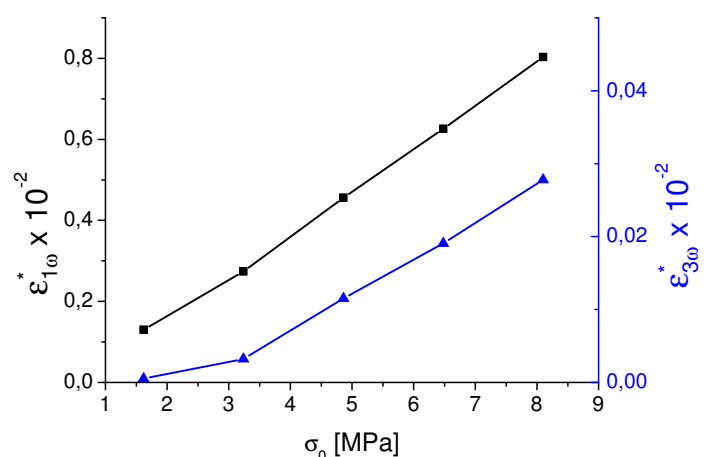

(a)

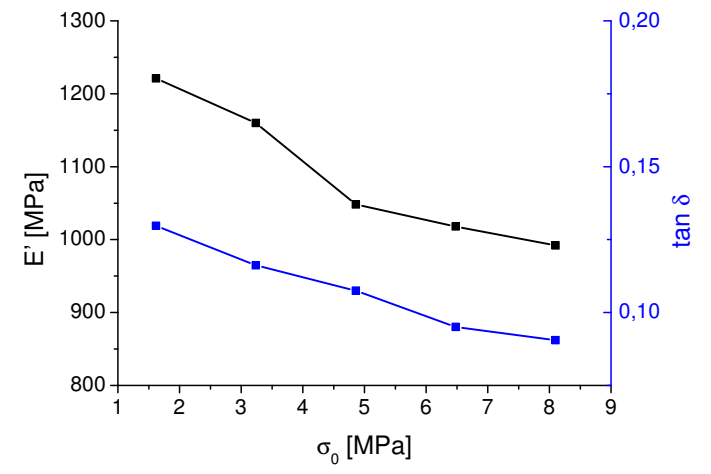

(b)

Abb. 4.15.: Plateauwerte für den Hochfeldbereich für $P M M A^{E}$ für verschiedene Anregungsamplituden bei einer Temperatur von $373 \mathrm{~K}$ (10K unterhalb von $T_{g}$.) a)Komplexe Dehnungsamplituden bei der fundamentalen und der dritten harmonischen der Anregungsfrequenz $\epsilon_{1 \omega}^{*}$ und $\epsilon_{3 \omega}^{*}$ b) Speichermodul und Verlust.

\subsubsection{Nichtlineares zeitabhängiges Verhaltens für $\mathrm{PMMA}^{G}$}

Die im vorherigen Abschnitt vorgestellten Messungen an $\mathrm{PMMA}^{E}$ wurden analog für das $\mathrm{PMMA}^{G}$ durchgeführt und sollen in diesem Teil der Arbeit gezeigt werden. Zunächst wird auch hier die Temperaturabhängigkeit behandelt und im Anschluss der Einfluss der externen Spannungsamplitude. Es wurden auch für das PMMA ${ }^{G}$ die Zeitabhängigkeit der Nichtlineraität mit dem NHN-Messprotokoll, für verschiedene Temperaturen bei einer Frequenz von $1 \mathrm{~Hz}$ untersucht. Die angelegten Spannungsamplituden betrugen 2,8MPa in den Niedrigfeldbereichen und 16,8MPa in den Hochfeldbereichen. Dies ist eine etwa dreimal so große Spannung, wie in den Messungen für $\mathrm{PMMA}^{E}$.. Dies liegt daran, dass die gleichen Kraftamplituden verwendete wurden, jedoch eine andere Probengeometrie für die $\mathrm{PMMA}^{G}$-Proben verwendet wurde. Die Abbildung 4.16 zeigt die komplexe Dehnungsamplitude $\epsilon_{1 \omega}^{*}$ für PMMA ${ }^{G}$ bei verschiedenen Temperaturen. Die entsprechende Dehnungsamplitude bei der 3 . Harmonischen ist in der Abbildung 4.17 abgebildet.

Im Gegensatz zu PMMA ${ }^{E}$ zeigt sich die Zeitabhängigkeit von $\epsilon_{1 \omega} *$ und $\epsilon_{3 \omega} *$ weniger stark und ist nur für die Temperatur von $383 \mathrm{~K}$ sehr ausgeprägt. Bei dieser Temperatur zeigt sich dann das schon für $\mathrm{PMMA}^{E}$ beobachtete Verhalten. Insbesondere zeigt $\epsilon_{3 \omega}^{*}$ am Ende des Hochfeldbereiches noch eine starke Steigung und ist damit noch nicht im Bereich eines Gleichgewichtszustandes.

Zunächst sollen auch hier die Temperaturabhängigkeit der Plateauwerte des Hochfeldbereiches ausgewertet werden. Da sich während der Messung in diesem Bereich keine Sättigung eingestellt hat, also kein „steady state“ erreicht wurde, wurden auch hier die Plateauwerte als Durchschnittt aus den letzten 100 Perioden des Hochfeldbereiches bestimmt. Die Abbildung 4.18a zeigt die Plateauwerte der komplexen 


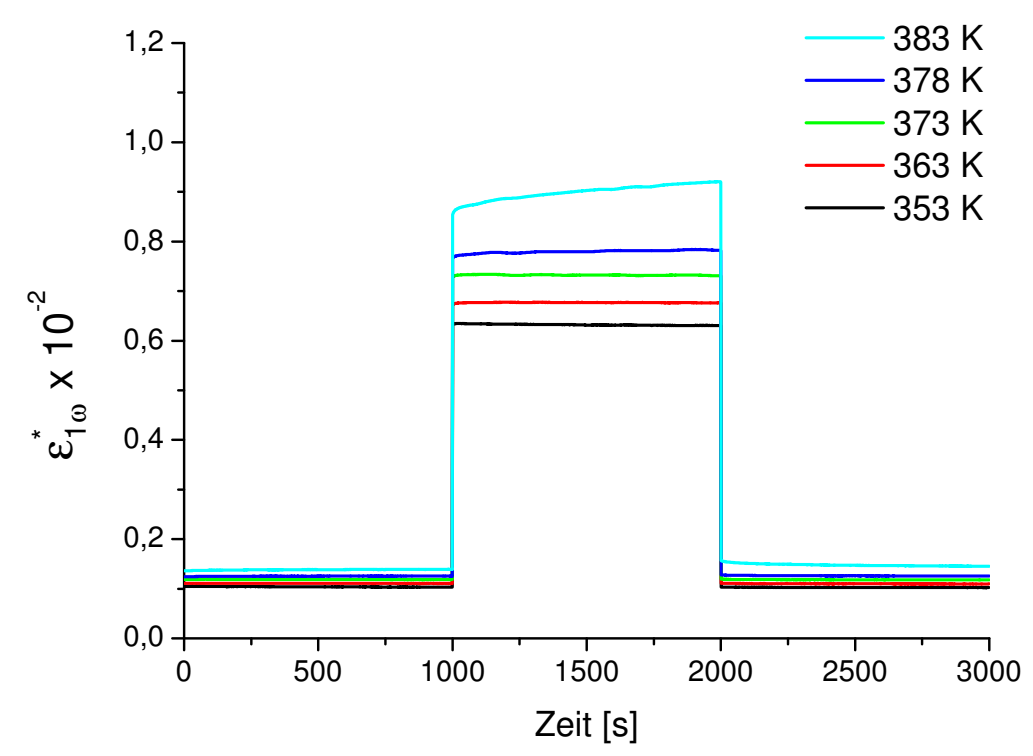

Abb. 4.16.: Erste harmonische Dehnungsamplitude $\epsilon_{1 \omega}^{*}$ für verschiedene Temperaturen für $P M M A^{G}$. Die Anregungsspannung im Niedrigfeldbereich betrug jeweils $\sigma_{0}=2,8 \mathrm{MPa}$ und im Hochfeldbereich $\sigma_{0}=16,8 \mathrm{MPa}$. Die verwendete Frequenz ist $1 \mathrm{~Hz}$.

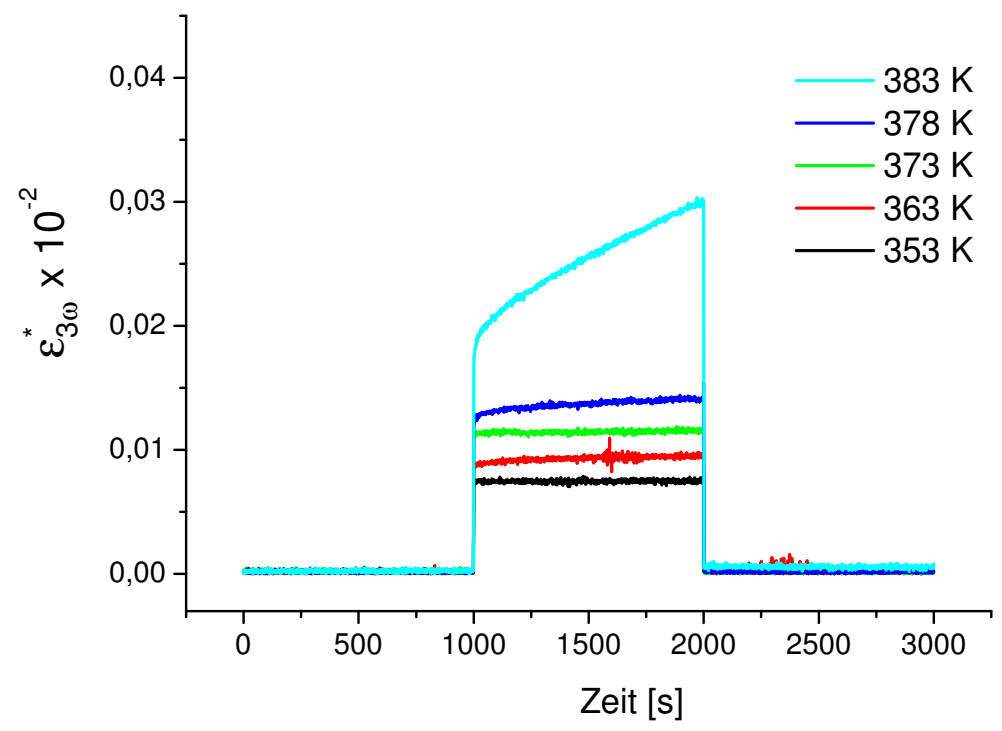

Abb. 4.17.: Dritte harmonische Dehnungsamplitude $\epsilon_{3 \omega}^{*}$ für verschiedene Temperaturen für PMMAG . Die Anregungsspannung im Niedrigfeldbereich betrug jeweils $\sigma_{0}=2,8 \mathrm{MPa}$ und im Hochfeldbereich $\sigma_{0}=16,8 \mathrm{MPa}$. Die verwendete Frequenz ist $1 \mathrm{~Hz}$. 


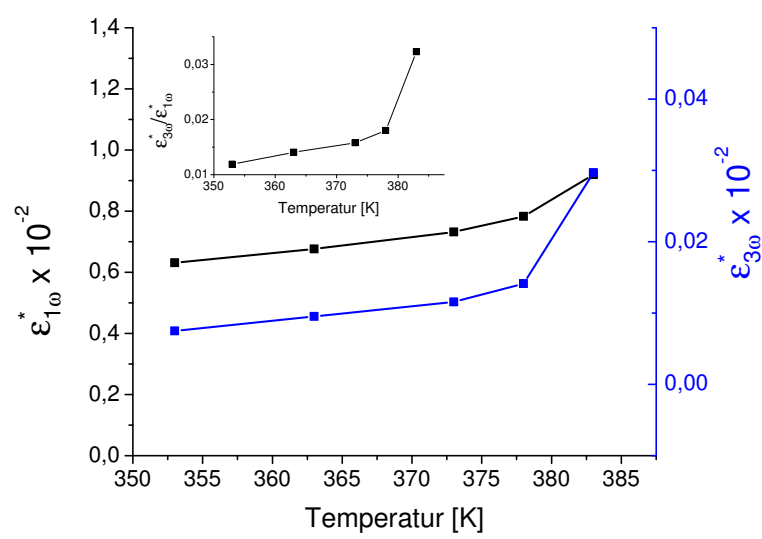

(a)

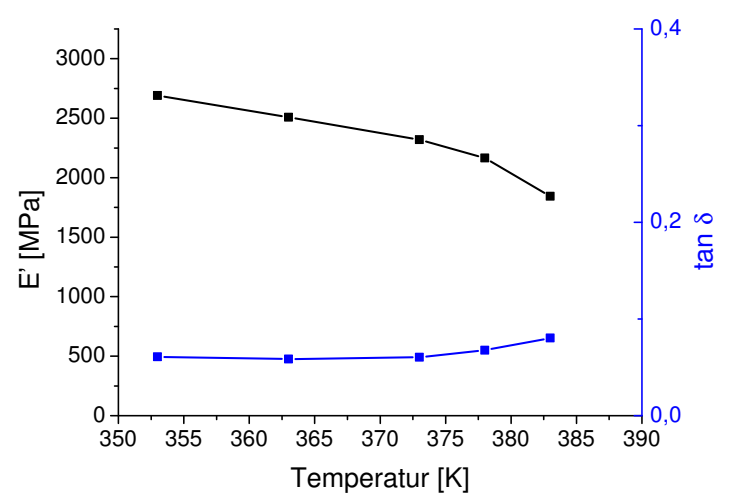

(b)

Abb. 4.18.: Temperaturabhängigkeit der Plateauwerte für den Hochfeldbereich für $P M M A^{G}$. a) Komplexe Dehnungsamplituden bei der fundamentalen und der dritten Harmonischen der Anregungsfrequenz $\epsilon_{1 \omega}^{*}$ und $\epsilon_{3 \omega}^{*}$. Das Inset zeigt die Temperaturabhängigkeit des Verhältnisses $\epsilon_{3 \omega}^{*} / \epsilon_{1 \omega}^{*}$ b) Speichermodul und Verlust. Die gezeigten Werte wurden aus den letzten 100 Perioden des Hochfeldbereiches bestimmt. In diesem Bereich hatte die Anregung eine Spannungsamplitude von $16,8 \mathrm{MPa}$ bei einer Frequenz von $\omega=1 \mathrm{~Hz}$.

Dehnungsamplituden bei der fundamentalen und der 3. harmonischen Frequenz für verschiedene Temperaturen. Die entsprechenden Plateauwerte des Speichermoduls E' und des $\tan \delta$ sind in Abbildung 4.18 dargestellt.

Analog zu dem für $\mathrm{PMMA}^{E}$ beobachteten Verhalten zeigt sich auch für $\mathrm{PMMA}^{G}$ der Onset des $\alpha$-Peaks in den Messungen. Bei Annäherung an $T_{g}$ steigen beide Dehnungsamplituden $\epsilon_{1 \omega}^{*}$ und $\epsilon_{3 \omega}^{*}$. Dieser Anstieg ist aber weniger deutlich ausgeprägt als für $\mathrm{PMMA}^{E}$. Auch der Verlauf von E' und $\tan \delta$ zeigt das erwartete Verhalten. E' steigt bei Annähreung an den Glasübergang an, dabei wächst auch simultan der Verlust $(\tan \delta)$. Im Gegensatz zu dem PMMA von Evonik ist dieser Effekt jedoch nicht so deutlich ausgeprägt. Dies ist zu erwarten, da die Temperaturscans aus Abschnitt 4.1 schon gezeigt haben, dass der Glasübergang für das PMMA von Goodfellow schärfer ausgeprägt ist und relativ abrupt mit der Temperatur einsetzt. Das bedeutet, dass die im PMMA ${ }^{E}$ enthaltenen Zusätze wahrscheinlich einen Einfluss auf das mechanische Verhalten haben und sich dies auch in den nichtlinearen Messungen manifestiert.

Auch $\mathrm{PMMA}^{G}$ wurde mit verschiedenen Spannungsamplituden $\sigma_{0}$ im Hochfeldbereich gemessen. Dabei wurde auch wie bei PMMA ${ }^{E}$ bei einer Temperatur von $10 \mathrm{~K}$ unterhalb von $T_{g}(T=378 \mathrm{~K})$ gemessen und eine Frequenz von $1 \mathrm{~Hz}$ verwendet. Der zeitliche Verlauf der komplexen Dehnungsamplituden $\epsilon_{1 \omega}^{*}$ und $\epsilon_{3 \omega}^{*}$ sind in den Abbildungen 4.19 und 4.20 dargestellt.

Im Gegensatz zu den entsprechenden Messungen an $\mathrm{PMMA}^{E}$ zeigt sich lediglich für die beiden größten Spannungsamplituden $\left(\sigma_{0}=22,4 \mathrm{MPa}, \sigma_{0}=28 \mathrm{MPa}\right)$ eine 


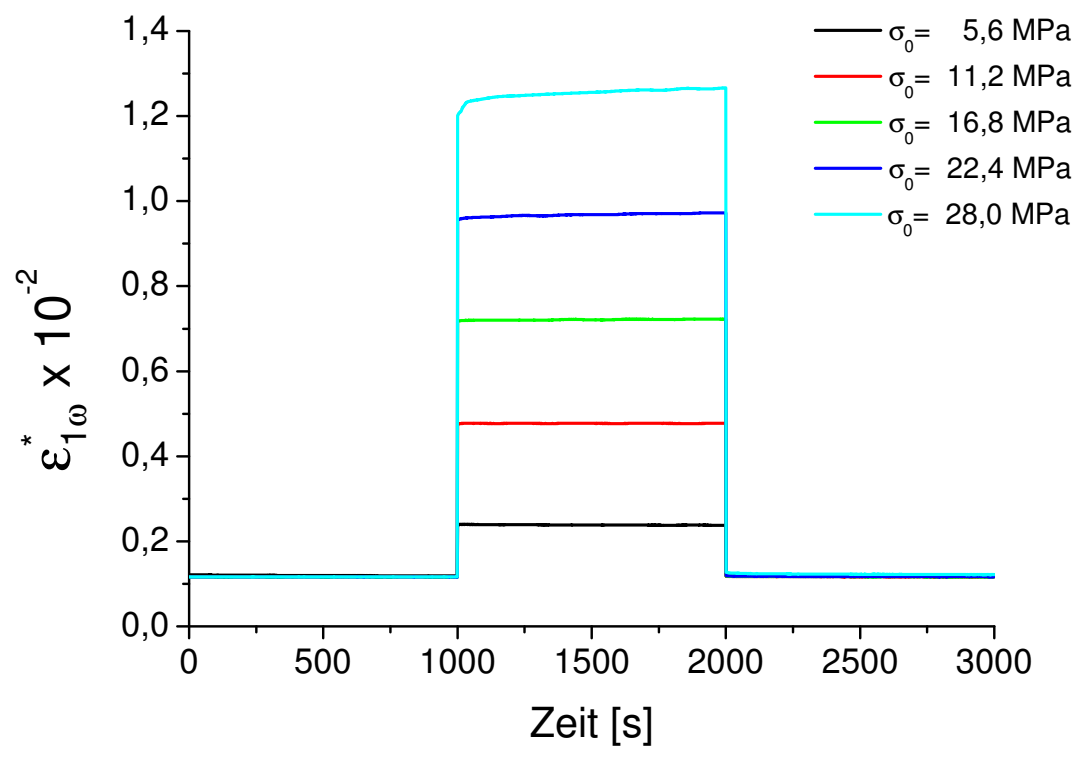

Abb. 4.19.: Erste harmonische Antwort-Amplitude $\epsilon_{1 \omega}^{*}$ für verschiedene dynamische Spannungen im Hochfeldbereich für $P M M A^{G}$ bei $T=373 \mathrm{~K}$. Die Spannungsamplitude in den Niedrigfeldbereichen beträgt 2,8 MPa und es wurde eine Frequenz von $1 \mathrm{~Hz}$ verwendet.

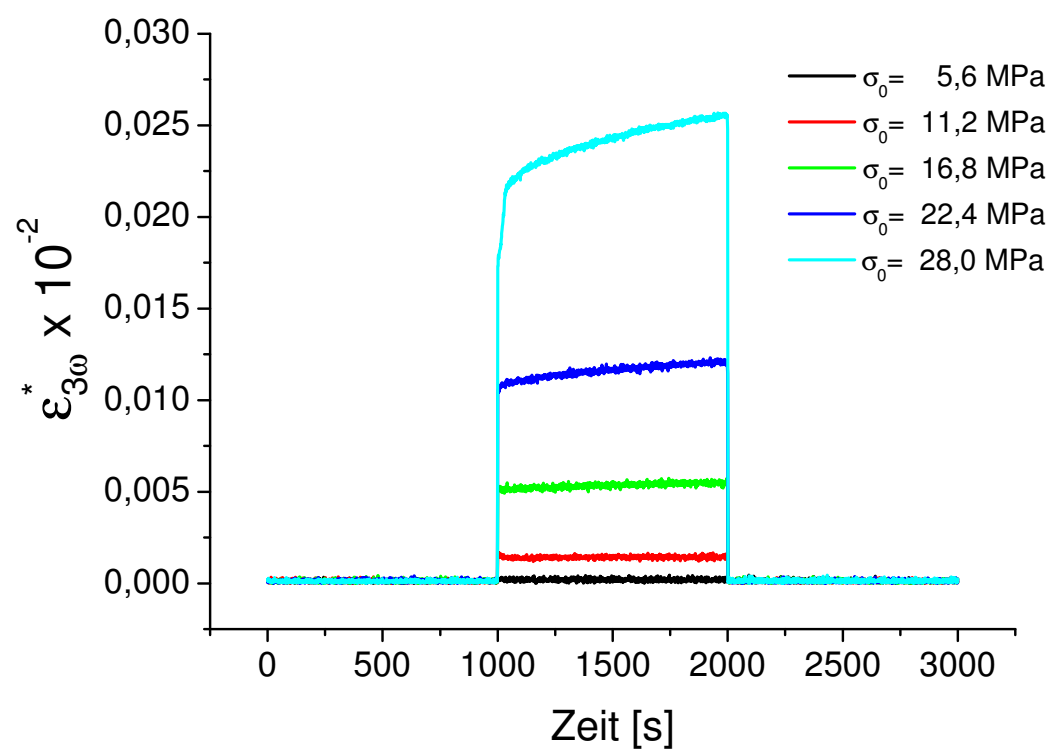

Abb. 4.20.: Dritte harmonische Antwort-Amplitude $\epsilon_{3 \omega}^{*}$ für verschiedene dynamische Spannungen im Hochfeldbereich für $P M M A^{G}$ bei $T=373$ K. Die Spannungsamplitude in den Niedrigfeldbereichen beträgt 2,8 MPa und es wurde eine Frequenz von $1 \mathrm{~Hz}$ verwendet. 


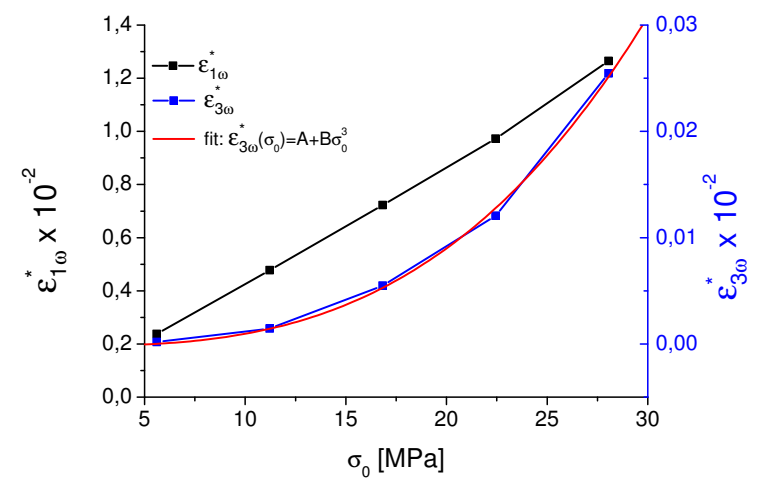

(a)

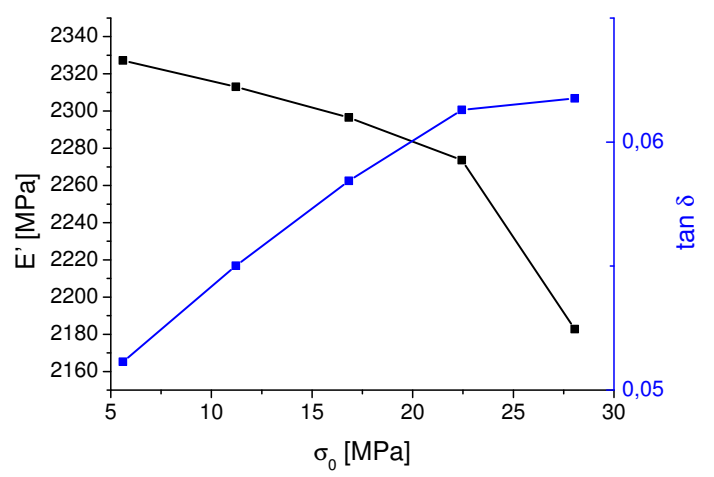

(b)

Abb. 4.21.: Plateauwerte für den Hochfeldbereich für $P M M A^{G}$ in für verschiedene Spannungsamplituden bei einer Temperatur von $378 \mathrm{~K}$.

ausgeprägte Zeitabhängigkeit in $\epsilon_{1 \omega}^{*}$ und $\epsilon_{3 \omega}^{*}$. Dies liegt daran, dass $T_{g}$ hier schärfer einsetzt und einen schmaleren Temperaturbereich abdeckt. Um einen ähnlich starken Effekt wie für PMMA ${ }^{E}$ zu erhalten, könnte die Messung bei einer höheren Temperatur durchgeführt werden. Analog zu den Messungen an $\mathrm{PMMA}^{E}$ zeigt auch hier $\epsilon_{1 \omega}^{*}$ am Ende des Hochfeldbereiches ein Plateau, während dazu im Gegensatz $\epsilon_{3 \omega}^{*}$ noch eine eindeutige Steigung am Ende der Hochfeldphase aufweist. Die Plateauwerte der komplexen Dehnungsamplituden im Hochfeldbereich sind in Abhängigkeit von $\sigma_{0}$ in der Abbildung 4.21a dargestellt. Dabei zeigt sich für $\epsilon_{1 \omega} *$ wie erwartet ein nahezu linearer Verlauf und für $\epsilon_{3 \omega^{*}}$ die erwartete kubische Abhängigkeit nach Gleichung 2.25. Die Plateauwerte für den Speichermodul und den Verlust sind in der Abbildung $\overline{4.21} \mathrm{~b}$ dargestellt. Sie zeigen sehr schön den erwarteten Verlauf. Der Speichermodul sinkt und simultan steigt der Verlust an. Bei der analogen Messung für PMMA ${ }^{E}$ konnte dieses Verhalten nicht beobachte werden (vergl. Abbildung 4.15b).

\subsubsection{Quantifizierung der nichtlinearen Effekte über die Nachgibiegkeiten höherer Ordnung}

Messungen mit verschiedener Spannungsamplitude bieten die Möglichkeit zu überprüfen, ob die kubischen Nachgiebigkeiten, die in der Theorie beschriebene quadratische Abhängigkeit vom externen Spanungsfeld zeigen. Es soll dabei insbesondere die Nachgiebigkeit der 3. Harmonischen $C_{3}^{*(3)}$ mit der Nachgiebigkeit dritter Ordnung bei der fundamentalen Frequenz $\left(C_{1}^{*(3)} \propto \Delta \ln C\right)$ verglichen werden. Die Abbildung 4.22 zeigt die nichtlinearen Nachgiebigkeiten bei der Fundamentalen und der 3. harmonischen Frequenz $\Delta \ln C_{1} \propto C_{3}^{(1)}$ und $C_{3}^{(3)} / C_{1}$, jeweils normiert auf die lineare Nachgiebigkeit $C_{1}$, für verschiedene Spannungsamplituden. Dabei sind die quadrierten Werte für das Feld aufgetragen, so dass die erwartete quadratische Abhängigkeit 
direkt durch einen linearen Fit sichtbar wird.

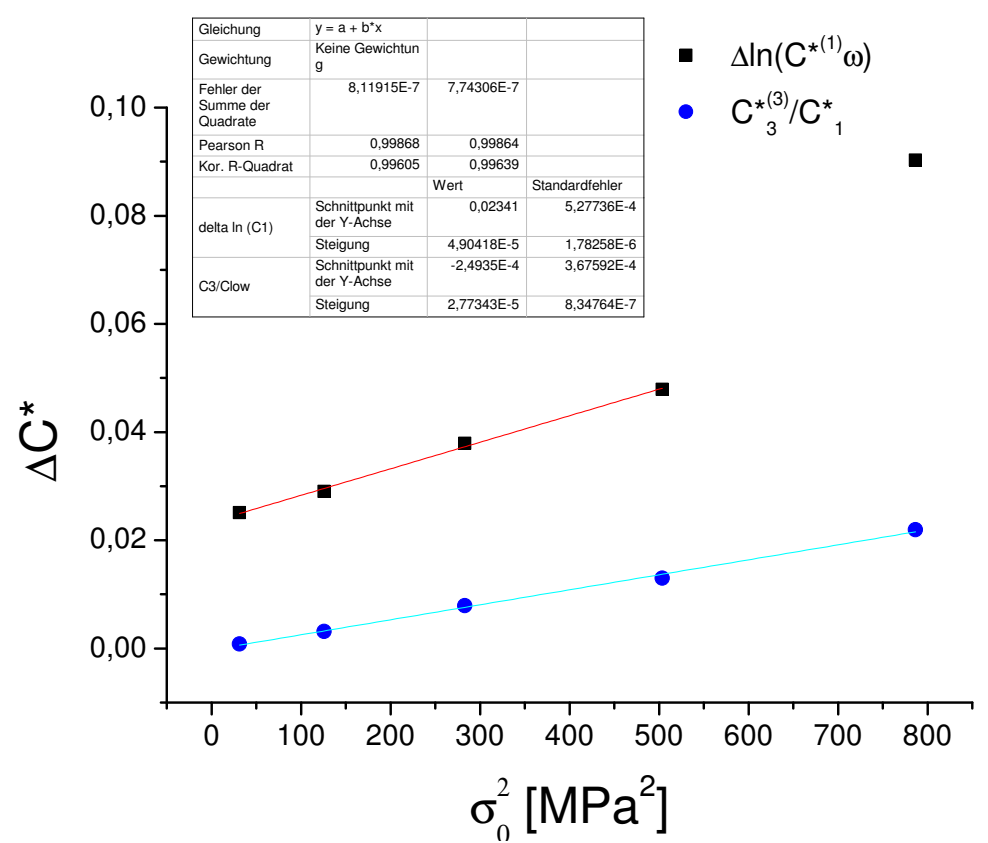

Abb. 4.22.: Komplexe Nachgiebigkeiten $C_{3}^{*(1)}$ und $C_{3}^{*(3)}$ normiert auf die fundamentale Nachgiebigkeit im kleinen Feld für PMMA ${ }^{G}$ bei $T=378 \mathrm{~K}$.

Es zeigt sich eindeutig für beide Nachgiebigkeiten dritter Ordnung die erwartete quadratische Abhängigkeit. Die aus den Fits erhaltenen Steigungen betragen ca. $5 \cdot 10^{-5} \mathrm{MPa}^{-2}$ für $\Delta \ln C_{1}$ und $2,8 \cdot 10^{-5} \mathrm{MPa}^{-2}$ für $C_{3}^{*(3)}$. Folglich wächst der nichtlineare Beitrag bei der fundamentalen Frequenz ungefähr doppelt so schnell an wie der nichtlineare Beitrag bei der dritten harmonischen Freauenz.

\subsubsection{Zeitkonstanten}

Die im vorherigen Abschnitt gezeigten Daten für die komplexen Dehnungsamplituden $\epsilon_{1 \omega}^{*}$ und $\epsilon_{3 \omega}^{*}$ wurden mit einer KWW-Funktion gefittet. Dabei wurde der Streckfaktor $\beta$ mit dem Wert 0,34 aus [69] konstant gesetzt. Die daraus bestimmten Werte für die Relaxationszeit sind in der Abbildung 4.23 für $\mathrm{PMMA}^{E}$ jeweils für unterschiedliche Temperaturen auf der linken Seite und für die verschiedenen Kraftamplituden auf der rechten Seite dargestellt. Dabei zeigt sich zunächst eine Zunahme von $\tau_{K W W}$ mit der Temperatur. Bei einer Temperatur von $373 \mathrm{~K}\left(T_{g}-10 K\right)$ jedoch wird $\tau_{K W W}$ kleiner mit steigender Temperatur. Aufgrund der thermischen Nähe zu $T_{g}$, kann angenommen werden, dass sich in dem Absinken der Relaxationszeit, die Physik des Glasüberganges niederschlägt. Die Werte, die für $\tau_{K W W}$ für verschieden Spannungsamplituden bestimmt wurden zeigen keinen systematischen Trend, sondern streuen eher um einen konstanten Wert. 


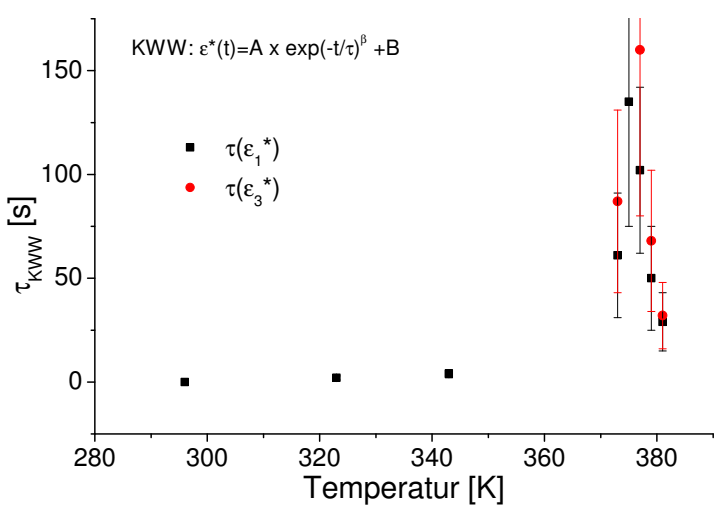

(a)

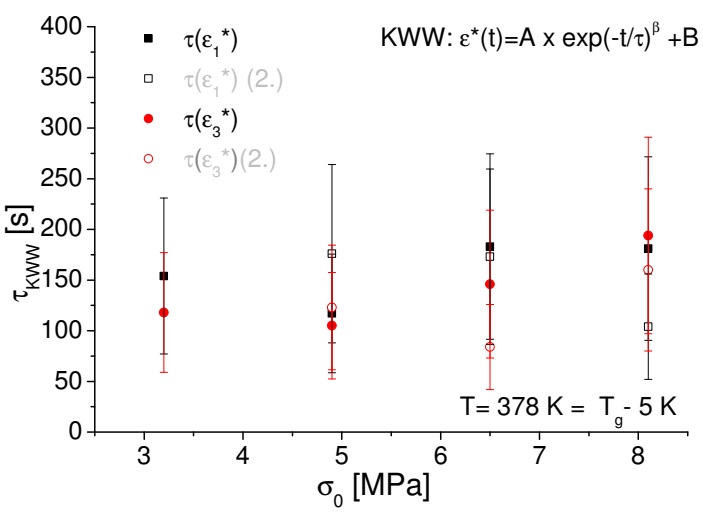

(b)

Abb. 4.23.: Relaxationszeit $\tau_{K W W}$ bestimmt aus der Zeitabhängigkeit der fundamentalen und der 3.harmonischen Dehnungsantwort im Hochfeldbereich für PMMA ${ }^{E}$ bei (a) verschiedenen Temperaturen und (b) verschiedenen Spannungsamplituden $\sigma_{0}$

Für PMMA ${ }^{G}$ sind die Werte für $\tau_{K W W}$ in der Abbildung 4.24 zusammengefasst. Es wird für dieses PMMA lediglich ein Ansteigen, von $\tau_{K W W}$ beobachtet. Mit steigender Spannungsamplitude sinkt $\tau_{K W W}$. Die Aussagekraft der hier bestimmten Abhängigkeiten sind jedoch eingeschränkt, aufgrund der wenigen Datenpunkte. Zudem sind auch die Absolutwerte für $\tau_{K W W}$ nicht verlässlich, da die zu fittenden Kurven von einerseits von dem in Abschnitt 3.3.6 beschriebenen Effekt der Temperaturregelung überlagert sind. Insbesondere war jedoch während der Messungen noch keine Sättigung erreicht. Trotzdem sind diese Werte als aussagekräftiger zu bewerten, da dieses PMMA eine höhere Reinheit aufweist und frei von Zusätzen ist.

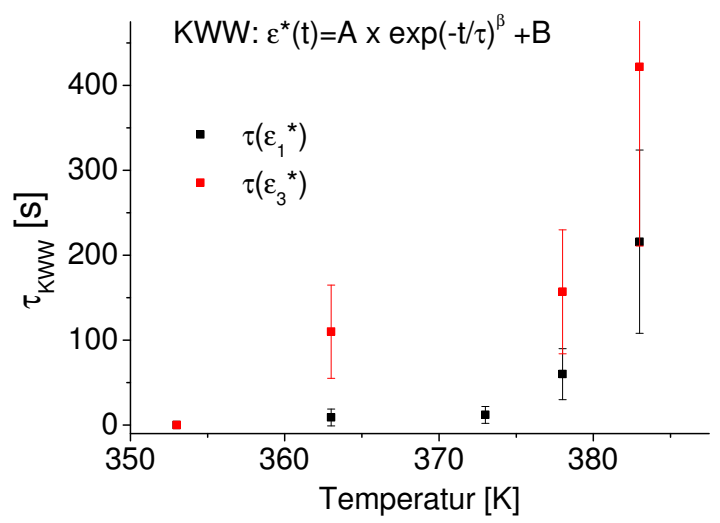

(a)

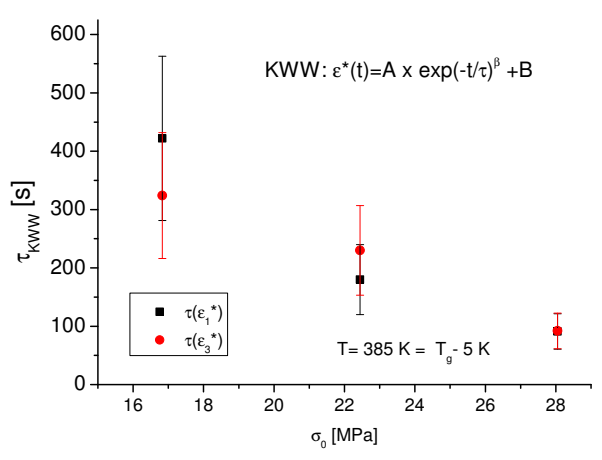

(b)

Abb. 4.24.: Relaxationszeit $\tau_{K W W}$ bestimmt aus der Zeitabhängigkeit der fundamentalen und der 3. harmonischen Dehnungsantwort im Hochfeldbereich für PMMA ${ }^{G}$ bei (a) verschiedenen Temperaturen und (b) verschiedenen Spannungsamplituden $\sigma_{0}$ 


\subsubsection{Zusammenfassung der Ergebnisse}

Es wurden zwei verschieden PMMA-Systeme mit mechanischer Spektroskopie bei großen dynamischen Spannungen untersucht. Dabei lag der Fokus darauf, die Methode $\mathrm{zu}$ entwickeln und $\mathrm{zu}$ untersuchen, welche neuen Informationen gewonnen werden können. Das Experiment ist eine dynamisch mechanische Analyse bei großen externen Anregungen, mit dem das nichtlineare mechanische Verhalten von amorphen Materialien charakterisiert werden soll. Zunächst wurden dafür zwei PMMASysteme mithilfe eines gewöhnlichen Temperaturscans im DMA auf ihr temperaturabhängiges mechanisches Verhalten untersucht. Dabei zeigten beide Materialien jeweils einen gut augeprägten Glasübergang sowohl im tan $\delta$, als auch im Speichermodul. Die Werte für die Glastemperaturen wurden jeweils aus dem Wendepunkt im Speichermodul bestimmt: $T_{g}\left(P M M A^{E}\right)=383 \mathrm{~K}$ und $T_{g}\left(P M M A^{G}\right)=390 \mathrm{~K}$. Die beiden PMMA unterscheiden sich dadurch, dass das $P M M A^{G}$ eine besonders hohe Reinheit aufweist. Dies manifestiert sich in den Temperaturscans in der geringeren Breite des Glasüberganges von $P M M A^{G}$. Es ist anzunehmen, dass das PMMA von Evonik Zusätze, wie beispielsweise Weichmacher enthält, die zu einer Verbreiterung des Glasüberganges führen. Der Einfluss des unterschiedlich breiten Glasüberganges zeigte sich auch im nichtlinearen Verhalten der Proben. Anhand von Messungen an $\mathrm{PMMA}^{E}$ konnte der Übergang zum nichtlinearen Regime beobachtet werden. Dies konnte mithilfe einer Lissajous-Auftragung visualisiert werden. Fourierspektren der Anregung und der Antwort zeigten, dass das Anregungssignal monofrequent ist und im Antwortsignal die erwarteten nichtlinearen Beiträge bei den Vielfachen der Anregungsfrequenz auftreten. Für verschiedene Temperaturen wurde das Amplitudenverhältnis $A_{3 \omega} / A_{1 \omega}$ gegen das Quadrat der Spannungsamplitude $\sigma_{0}^{2}$ aufgetragen und die Steigung aus linearen Fits bestimmt. Diese Steigung zeigt ein exponentielles Wachstum mit der Temperatur mit einer Konstante von 1/4. Die Zeitabhängigkeit der nichtlinearen Effekte konnte mit einem NHN-Experiment gezeigt werden. Diese Experimente wurden für beide PMMA-Systeme durchgeführt. Dabei wurde einerseits bei verschiedenen Temperaturen direkt unterhalb von $T_{g}$ gemessen und andererseits wurde bei konstanter Temperatur die Spannungsamplitude im Hochfeld variiert. Für die temperaturabhängigen Messungen zeigte sich für beide PMMA, dass ab einer bestimmten Temperatur zeitabhängiges Verhalten sowohl fü $\epsilon_{1 \omega}^{*}$, als auch für $\epsilon_{3 \omega}^{*}$ in der Hochfeldphase beobachtet wird. Dabei zeigt jeweils $\epsilon_{1 \omega}^{*}$ nahezu eine Sättigung am Ende des Hochfeldbereiches, während $\epsilon_{3 \omega}^{*}$ noch eine starke Steigung aufweist. Dabei liegt die Temperatur, ab welcher die Zeitabhängigkeit beobachtet wird für PMMA ${ }^{E}$ bei $373 \mathrm{~K}\left(10 \mathrm{~K}\right.$ unterhalb von $T_{g}$ ) und für PMMA ${ }^{G}$ bei $383 \mathrm{~K}$ (7K unterhalb von $T_{g}$ ). Es wurden aus den letzten 100 Perioden im Hochfeld jeweils Plateauwerte bestimmt. Diese Plateauwerte zeigen für die Dehnungsamplituden $\epsilon_{1 \omega}^{*}$ und $\epsilon_{3 \omega}^{*}$ jeweils einen Anstieg mit der Temperatur. Dabei steigt jedoch $\epsilon_{3 \omega}^{*}$ stärker an, so dass das Verhältnis $\epsilon_{3 \omega}^{*} / \epsilon_{1 \omega}^{*}$ ebenfalls mit der Temperatur ansteigt. Aus den Plateauwerten wurden zudem Speichermodul und Verlust bestimmt um das Experiment auf Konsistenz zu prüfen. Es wurde hier für beide PMMA das erwartete und aus den Temperaturscans bekannte Verhalten gefunden. Mit steigender Tempe- 
ratur und größerer Nähe zu $T_{g}$ sinkt der Speichermodul und simultan zeigt der Verlust einen Anstieg. Die NHN-Messungen mit verschiedener Spannungsamplitude im Hochfeldbereich konnten ebenfalls für beide PMMA-Systeme zeitabhängiges Verhalten beobachten. Dabei wurden die Messungen jeweils 10K unterhalb der jeweiligen Glasübergangstemperatur durchgeführt. Daher zeigt sich auch bei PMMA ${ }^{E}$ schon bei kleineren Spannungsamplituden das zeitabhängige Verhalten, da dieses PMMA einen sehr frühen Onset des Glasüberganges zeigt. Die Zeitabhängigkeit zeigt auch hier einen qualitativen Unterschied für $\epsilon_{1 \omega}^{*}$ und $\epsilon_{3 \omega}^{*}$. Denn am Ende der Hochfeldphase zeigt $\epsilon_{3 \omega}^{*}$ auch hier noch eine signifikante Steigung, während $\epsilon_{1 \omega}^{*}$ nahezu gesättigt ist. Die Plateauwerte wurden ebenfalls über die 100 letzten Perioden bestimmt und in Abhängigkeit der Spannungsamplitude aufgetragen. Dabei zeigt sich ein großer qualitativer Unterschied zwischen den beiden PMMA-Systemen. Das PMMA ${ }^{G}$ zeigt das erwartete Verhalten nach Gleichung 2.25, denn $\epsilon_{1 \omega}^{*}$ zeigt das in erster Näherung erwartet lineare Verhalten und der Verlauf von $\epsilon_{3 \omega}^{*}$ kann mit einer kubischen Funktion gefittet werden. Dazu im Gegenatz wird für PMMA ${ }^{E}$ zwar ein nahezu lineares Verhalten für $\epsilon_{1 \omega}^{*}$ beobachtet, jedoch kann die kubische Abhängigkeit zwischen $\epsilon_{3 \omega}^{*}$ und $\sigma_{0}$ nicht beobachtet werden. Aus den Plateauwerten wurde auch hier wieder Speichermodul und Verlust bestimmt und es zeigt sich, dass erneut für $\mathrm{PMMA}^{G}$ die theoretischen Erwartungen bestätigt werden. Der Speichermodul zeigt einen Abfall, der von einem Anstieg im Verlust begleitet wird. Im Gegensatz dazu wird für PMMA $^{E}$ ein Absinken von Speichermodul und Verlust beobachtet, was im Widerspruch zu den Erwartungen ist. Aus den zeitabhängigen Verläufen wurden mithilfe von KWW-Fits Zeitkonstanten bestimmt. Diese wachsen zunächst für beide PMMA mit der Temperatur an und zeigen dann für $\mathrm{PMMA}^{E}$ wieder ein Absinken. In Abhängigkeit der Spannungsamplitude zeigte sich für $\mathrm{PMMA}^{E}$ ein nahezu konstanter Wert und für $\mathrm{PMMA}^{G}$ konnte beobachtet werden ,dass $\tau_{K W W}$ mit steigender Spannungsamplitude kleiner wird. Da die Plateauwerte der spannungsabhängigen Messung an $\mathrm{PMMA}^{G}$ das erwartete Verhalten wiedergeben, wurden zusätzlich die nichtlinearen Nachgiebigkeiten bestimmt. Es zeigen beide sehr schön die lineare Abhängigkeit von $\sigma_{0}^{2}$. Dabei war die Steigung für die Nachgiebigkeit bei der dritten harmonischen Frequenz ungefahr halb so groß wie die des nichtlinearen Anteiles der Nachgiebigkeit bei der fundamentalen Frequenz. 


\subsection{Diskussion}

Es wurden zwei verschieden PMMA-Systeme auf ihr nichtlineares mechanisches Verhalten untersucht. Dabei lag der Fokus darauf, eine experimentelle Methode zu entwickeln und zu prüfen, ob die Ergebnisse die theoretischen Voraussagen erfüllen um schließlich neue Informationen gewinnen zu können. Das Experiment ist eine dynamisch mechanische Analyse bei großen externen Anregungen, mit dem das nichtlineare mechanische Verhalten von amorphen Materialien charakterisiert werden soll. Zunächst wurden dafür zwei PMMA-Systeme mithilfe eines gewöhnlichen Temperaturscans im DMA auf ihr temperaturabhängiges mechanisches Verhalten untersucht. Dabei zeigten beide Materialien jeweils einen gut augeprägten Glasübergang sowohl $\operatorname{im} \tan \delta$, als auch im Speichermodul. Die Werte für die Glastemperaturen wurden jeweils aus dem Wendepunkt im Speichermodul bestimmt: $T_{g}\left(P M M A^{E}\right)=383 \mathrm{~K}$ und $T_{g}\left(P M M A^{G}\right)=390 \mathrm{~K}$. Die beiden PMMA unterscheiden sich dadurch, dass das $P M M A^{G}$ eine besonders hohe Reinheit aufweist. Dies manifestiert sich in den Temperaturscans in der geringeren Breite des Glasüberganges von $P M M A^{G}$. Es ist anzunehmen, dass das PMMA von Evonik Zusätze, wie beispielsweise Weichmacher enthält, die zu einer Verbreiterung des Glasüberganges führen. Der Einfluss des unterschiedlich breiten Glasüberganges zeigte sich auch im nichtlinearen Verhalten der Proben, wie im Folgenden anhand der Ergebnisse aus Abschnitt $\underline{4}$ diskutiert werden soll.

\subsubsection{Detektion des nichtlinearen Verhaltens an $\mathrm{PMMA}^{E}$}

Zuerst soll der Übergang und die Detektion des nichtlineare Verhalten anhand von Messungen an $P M M A^{E}$ diskutiert werden. Für $P M M A^{E}$ wurden zunächst Experimente durchgeführt, die die Zeitabhängigkeit der nichtlinearen Beiträge nicht berücksichtigen. Dabei wurden jeweils bei unterschiedlichen Temperaturen unterhalb von $T_{g}$ für verschiedene externe Spannungsamplituden dynamisch mechanische Messungen durchgeführt. Eine erste Charakterisierung des Probenverhaltens erfolgte über eine Lissajous-Auftragung für eine Messung bei $353 \mathrm{~K}$. Dabei wurden jeweils für eine Periode die zeitabhängige Spannung gegen die Probendehnung aufgetragen. Anhand dieser Auftragung konnten die folgenden Effekte sichtbar gemacht werden. Bei kleinen Anregungsspanungen zeigte die Probe ein nahezu ideal elastisches Verhalten, und die Lissajouskurve zeigt einen Strich. Für höhere Spannungsamplituden konnte zunächst das Einsetzen dissipativer Prozesse beobachtet werden. Das bedeutet, die Lissajouskurve zeigt eine Ellipse, deren Flächeninhalt proportional zur dissipierten Energie ist. Dieser Bereich ist gekennzeichnet von linearem viskoelastischen Verhalten der Probe. Das bedeutet, es wird zwar Energie im Material verbraucht, jedoch finden keine irreversiblen plastischen Umformungen im Material statt [52]. Im Bild der potentiellen Energielandschaft bedeutet dies, dass es durch die hohe äußere Spannung zu einer Verkippung der Energiebarrieren kommt, so dass andere Subbasins in der PEL zugänglich sind. Die Spannung reicht jedoch nicht aus, 
die PEL soweit zu verkippen, dass ein neues Metabasin erreichbar wäre. Bei noch höherer Spanung ist die Symmetrie der Lissajous Ellipse gestört und es kann nichtlineares viskoelastische Verhalten beobachtet werden. Dies entspricht dem Auftreten plastischer Prozesse und bedeutet, dass durch die hohe Spannung die lokalen Energiebarrieren soweit verschoben sind, dass sich das System in einen Bereich der PEL bewegt, der nach dem Entfernen der äußeren Spannung nicht mehr verlassen werden kann, da ohne das äußere Feld die Barrierenhöhe wieder so hoch ist, dass das System nicht in die ursprüngliche Konfiguration zurückkann. Dies zeigt sich im Experiment durch die irreversiblen plastischen Verformungen der Probenkörper. Da die Lissajousdarstellung jedoch keine quantitative Beschreibung der nichtlinearen Beiträge gestattet, wurde mithilfe von Fouriertransformationen die Messdaten genauer analysiert. Es konnte anhand dieser Auswertung zunächst beobachtet werden, dass das Anregungssignal außer bei der fundamentalen Frequenz keine signifikanten Beiträge bei anderen Frequenzen liefert. Zwar zeigte das Fourierspektrum kleine Beiträge bei der zweiten und bei der vierten Harmonischen der angelegten Frequenz, jedoch liegt deren Amplitude unterhalb von 0,1\% der Fundamentalen. Damit ist gewährleistet, dass das Anregungssignal nahezu monofrequent ist und die Detektion von höheren Harmonischen in der Signalantwort auf das nichtlineare Verhalten des Materials zurückzuführen sind. Die simultan aufgezeichnete Dehnungsantwort des $\mathrm{PMMA}^{E}$ zeigt hingegen eindeutig eine Oszillation, die keiner einfachen Sinusschwingung entspricht. Das Fourierspektrum dieser Antwortfunktion zeigt Beiträge bei den Vielfachen der Grundfrequenz. Insbesondere sind, wie aus der Theorie erwartet, signifikante Beiträge bei den ungeraden Harmonischen zu detektieren. Dies zeigt eindeutig, dass das Experiment in der Lage ist, das nichtlineare Verhalten zu zeigen und die verschiedenen harmonische Beiträge zu quantifizieren. Zusätzliche Beiträge bei den geraden Harmonischen können auf durch den Einbau hervorgerufene Vorspannungen zurückgeführt werden.

Da sich die Nichtlinearität außer in der Fundemntalen am deutlichsten in den Beiträgen dritter Ordnung manifestiert, wurde zunächst die Amplitude der Dehnungsantwort bei der 3. harmonischen Frequenz untersucht. Um einen Überblick zu bekommen, welche Temperaturen und welche Anregungsspannungen geeignet sind, das nichtlineare Verhalten im PMMA $^{E}$ zu detektieren wurde systematisch bei verschiedenen Temperaturen die Spannungsamplitude erhöht. Als Maß für die auftretende Nichtlinearität wurde das Verhältnis der dritten Harmonischen Amplitude zur Amplitude bei der fundamentalen Frequenz $A_{3 \omega} / A_{1 \omega}$ genutzt. Dieses Verhältnis wird in vielen Veröffentlichungen zur Quantifizierung der Nichtlinearität im Material herangezogen [49],[48],[51]. In erster Näherung wird erwartet, dass dieses Verhältnis nach Gleichung (2.29) quadratisch mit der Amplitude der Anregungsspannung wächst. Dieser Zusammenhang konnte für den Temperaturbereich zwischen $353 \mathrm{~K}$ und $368 \mathrm{~K}$ beobachtet werden. Dabei zeigt der Proportionalitätsfaktor $b$ aus $A_{3 \omega} / A_{1 \omega} \propto b \sigma_{0}^{2}$ eine eindeutige Temperaturabhängigkeit. Diese Temperaturabhängigkeit kann mit einer Exponentialfunktion gefittet werden. Dabei ergibt sich die Konstante für das exponentielle Wachstum zu ca. 1/4. Dabei ist jedoch zu beachten, dass der Offset 
der Exponentialfunktion nicht zu vernachlässigen ist, also $\ln (b)$ nicht linear mit der Temperatur wächst, sondern $\ln \left(b-b_{0}\right)$. Aus diesen ersten Messungen kann festgestellt werden, dass über die externe Spannungsamplitude die nichtlinearen Beiträge erhöht werden könnnen. Insbesondere zeigen die nichtlinearen Beiträge die erwartete Proportionalität zum Quadrat der Spannungsamplitude. Andererseits, zeigt sich ein Anstieg der Nichtlinearität auch mit der Temperatur. Dabei wird jedoch eine stärkere Abhängigkeit als für die Spannungsamplitude gefunden. Das hier beobachtete exponentielle Verhalten ist auf eine starke Zunahme der Dynamik bei Annäherung an $T_{g}$ zurückzuführen. Es ist jedoch deutlich, dass der Übergang zum nichtlinearen Regime zwar sowohl durch externe Spannung als auch durch die Temperatur getrieben werden können, diese jedoch dabei unterschiedliche Abhängigkeiten zeigen. Dies liegt daran, dass sich eine Temperaturerhöhung im Bild der PEL so auswirkt, dass Barrieren überwunden werden können, während eine Erhöhung der Spannung zu einer Verkippung der Barrieren führt. Daher ist es von besonderem Interesse neben der Temperaturabhängigkeit der nichtlinearen Prozesse ebenso deren Spannungsabhängigkeit zu untersuchen. Insbesondere gibt es zudem Hinweise darauf, dass sich auch die Fluktuationen in der PEL qualitativ unterscheiden. Denn Martens et al. konnten zeigen, dass mechanische und thermische Fluktuationen zu verschiedenen Statistiken bezüglich des Überwindens von Barrieren in der PEL führen [67].

\subsubsection{Zeitabhängige Messungen}

Da es sich beim Glasübergang um einen dynamischen Prozess handelt, sind zeitaufgelöste Messungen von besonderem Interesse. Daher wurden Experimente durchgeführt, bei denen die Signale periodenweise fouriertransformiert wurden, um so die zeitliche Entwicklung der Fourierspektren beobachten zu können. Dafür wurde ein NHN-Experiment durhgeführt. Dabei wird die Probe zunächst mit kleiner Spannungsamplitude im linearen Regime angeregt und die Amplitude nach einer Anzahl von Perioden $N$ instantan erhöht. Nach N Perioden in diesem Hochfeld, wird die Spannungsamplitude wieder auf den Anfangswert redutiert. Dadurch kann auch der Zerfall der nichlinearen Prozesse beobachtet werden und zudem kann der Vergleich der beiden Niedrigfeldbereiche irreversible Verformungen sichtbar machen. Anhand dieser Experimente konnte eindeutig eine Zeitabhängigkeit mit einer guten Auflösung beobachtet werden. Im Gegensatz zu dielektrischen Messungen, bei denen die nichtlinearen Beiträge sehr klein sind $(\leq 0,5 \%$, [45]), zeigt sich hier das nichtlineare Signal mit einer eindeutigen Stärke. Insbesondere wurden höhere Harmonische bis zur 9. Ordnung detektiert, dabei traten auch hier die geraden Harmonischen auf.

Für die Untersuchung der Zeitabhängigkeit wurden in dieser Arbeit die komplexen Dehnungsamplituden $\epsilon^{*}$ herangezogen, da diese direkt aus den Rohdaten des Antwortsignals ermittelt werden können. Die typischen Messgrößen der Dynamisch mechanischen Analyse, Speicher- und Verlustmodul wurden für eine erste Analyse nicht verwendet, da die Bestimmung des Verlustwinkels eine Fehlerquelle darstellt, 
da jeweils nur für eine Periode die Phasenverschiebung bestimmt wird.

Es konnte für beide der PMMA-Systeme eindeutig eine Zeitabhängigkeit der nichtlinearen Antwort beobachtet werden. Eine Zeitabhängigkeit der nichtlinearen Prozesse kann bedeuten, dass eine tyische Zeitskala damit verknüpft ist. Im Bild des Box-Modells, welches für die dielektrische Spektroskopie entwickelt wurde, bedeutet das, dass das Material unabhängig von der Frequenz der Anregung nur auf einer bestimmten Zeitskala relaxieren kann, die aus dem Glasübergang stammt und der $\alpha$-Relaxationszeit entspricht. Denn $\tau_{\alpha}$ ist die Relaxationszeit der Matrix und führt zu der makroskopischen Materialantwort, unabhängig von der jeweiligen Anregungsfrequenz. Die Zeitabhängigkeit der nichtlinearen Antwort kann im Rahmen des Box-Modells über eine Erhöhung der fiktiven Temperatur erklärt werden. Die fiktive Temperatur ist dabei als diejenige Temperatur definiert, für die das Probensystem im linearen Fall die beobachtete Relaxationszeit zeigt. Da die Erhöhung der Temperatur damit verknüpft ist, wie schnell die Energie der Mode, die mit hoher Spannung angeregt wurde an das Phononenbad abgegeben werden kann, kommt es zu einer Zeitabhängigkeit. Denn für jede der einzelnen Moden im Material wird eine eigene Relaxationszeit angenommen und das gesamte Probenmaterial kann nur auf der Zeitskala der $\alpha$-Relaxation relaxieren [75]. Die in dieser Arbeit bestimmten Relaxationszeiten liegen im Bereich von $\tau_{\alpha}$ und sind damit in guter Übereinstimmung mit dem Modell.

Die zeitabhängigen Messungen wurden zunächst hinsichtlich der Plateauwerte ausgewertet. Es zeigte sich dabei, dass die Anzahl der im Experiment verwendeten Perioden nicht ausreichend ist, um eine Sättigung auf einen Antwortwert zu erreichen. Daher wurde als Plateauwert jeweils der Mittelwert der 100 letzten Perioden im Hochfeldbereich ausgewertet. Dabei wurden einerseits die Plateauwerte der komplexen Dehnungsamplituden $\epsilon_{1 \omega}^{*}$ und $\epsilon_{3 \omega}^{*}$ aufgetragen, als auch der mit Hilfe des Phasenwinkels $\delta$ bestimmte Speichermodul $E^{\prime}$, sowie der Verlustfaktor $\tan \delta$.

Zunächst sollen die Ergebnisse für das $\mathrm{PMMA}^{E}$ diskutiert werden. Dabei sollen erst die Temperaturabhängigkeit und anschließend die Spannungsabhängigkeit des Nichtlinearen Verhaltens diskutiert werden. Anschließend werden ebenfalls für PMMA ${ }^{G}$ diese beiden Abhängigkeiten diskutiert. Abschließend sollen die Ergebnisse für beide Probensysteme verglichen werden.

\subsubsection{Temperatur- und Spannungsabhängigkeit der Plateauwerte für PMMA $^{E}$}

Die Temperaturabhängigkeit für die komplexen Dehnungsamplituden zeigt für $1 \omega$ und $3 \omega$ jeweils einen starken Anstieg bei Annäherung an $T_{g}$. Dies entspricht dem Verhalten des Verlustes $\tan \delta$ und zeigt, dass die beobachteten nichtlinearen Effekte mit dem Einsetzen von dissipativen Prozessen verknüpft sind. Der Speichermodul zeigt das aus den einfachen Temperaturscans bekannte Verhalten und nimmt simul- 
tan mit dem Anstieg des Verlustes ab. Damit ist die hier beobachtete Temperaturabhängigkeit konsistent mit dem aus den Standard-Temperaturscans bekannten Verhalten. Damit ist gewährleistet, dass die Messmethode in diesem Punkt brauchbare Ergebnisse liefert. Hervorzuheben ist hierbei, dass für das $\mathrm{PMMA}^{E}$ der Abfall des Speichermoduls sehr gravierend ist. Er fällt von einem Wert von $2800 \mathrm{MPa}$ bei Raumtemperatur auf einen Wert unterhalb $500 \mathrm{MPa}$ bei $T=380 \mathrm{~K}$.

Das Experiment wurde ebenfalls mit unterschiedlicher Spannungsamplitude im Hochfeldbereich durchgeführt. Dabei konnte beobachtet werden, dass wie erwartet durch die Erhöhung von $\sigma_{0}^{H}$ das nichtlineare Regime erreicht werden kann. Dies äußert sich in den Messungen einerseits durch das Auftreten eines Signals bei $3 \omega$, als auch in der einsetzenden Zeitabhängigkeit von $\epsilon_{1 \omega}^{*}$, für Amplituden von $\sigma_{0}^{H} \geq 3,2 \mathrm{MPa}$. Für die Messungen bei verschiedener Spannungsamplitude wurde für PMMA ${ }^{E}$ zusätzlich noch der Anteil der Irreversibilität untersucht. Dafür wurden einige Messungen mit gleicher Spannungsamplitude nach einer Wartezeit wiederholt. Dabei zeigt sich, dass es einen Bereich der Spannungsamplituden gibt, bei denen die Messung und ihre Wiederholung direkt übereinander liegen $\left(\sigma_{0} \leq 4,9 \mathrm{MPA}\right)$. Für die Messungen bei höherer Spannungsamplitude hingegen zeigt sich jeweils für die zweite Messung mit gleichem $\sigma_{0}$, dass der nichtlineare Beitrag nun höher ist. Durch das Anregen im nichtlinearen Bereich scheint das Material weniger steif und die einzelnen Polymerketten sind beweglicher. Durch die Belastung mit der hohen Ampliutde kann man sich vorstellen, dass gewisse Pfade für die Molekülketten geschaffen sind, so dass beim zweiten Mal bei der Beanspruchung mit der gleich großen Amplitude die Moleküle sich leichter bewegen können und damit die komplexe Dehnungsantwort größer ist.

Für die Abhängigkeit der Plateauwerte von der externen Spannungsamplitude zeigt sich für PMMA ${ }^{E}$ wie erwartet ein nahezu linearer Verlauf in der komplexen Dehnungsamplitude der Fundamentalen und ein zunächst flacherer Verlauf für $\epsilon_{3 \omega}^{*}$. Erwartet wird für $\epsilon_{3 \omega}^{*}$ eine kubische Abhängigkeit von $\sigma_{0}$, was nicht eindeutig beobachtet wird, wobei jedoch die Anzahl der Datenpunkte für Schlussfolgerungen nicht ausreichend ist. Der Speichermodul $E^{\prime}$ zeigt ein Absinken, von $1250 \mathrm{MPa}$ auf $1000 \mathrm{MPa}$ mit der externen Spannungsamlitude. Diese Werte liegen schon weit unterhalb des Moduls, der bei Temperaturen deutlich unterhalb von $T_{g}$ einen Wert von $2800 \mathrm{MPa}$ liefert. Das bedeutet, dass das sich diese Messungen schon mitten im Glasübergang befindet. Im Vergleich dazu, zeigt der Verlust ebenfalls ein Absinken. Dies ist im Widerspruch zu dem erwarteten Verhalten. Warum der Verlust dieses Absinken zeigt könnte darauf zurückgeführt werden, dass das Material sehr nahe bei $T_{g}$ ist, was schon anhand des Speichermodules zu beobachten ist. Nahe bei $T_{g}$ führt eine externe Spannung zu einer plastischen Verformung des Probenkörpers. Da die Bereiche der Probe, die sich in der Einspannung befinden den größten Kräften ausgesetzt sind, werden sich diese Bereiche auch als erstes verformen. So eine Verformung kann dazu führen, dass die Probe nicht mehr fest eingespannt ist und stellt damit eine Fehlerquelle dar. Das bedeutet, bei Messungen der Nichtlinearität zwar nahe bei $T_{g}$ die größten Beiträge erwartet werden, aber zudem in diesem Be- 
reich der Fehler auch besonders groß werden kann. Damit ist für die Messungen der Temperaturbereich direkt beim Onset von $T_{g}$ am besten geeignet.

\subsubsection{Temperatur- und Spannungsabhängigkeit der Plateauwerte für PMMA $^{G}$}

Das $\mathrm{PMMA}^{G}$ wurde ebenfall im Hinblick auf die Temperaturabhängigkeit der beobachteten nichtlinearen Effekte untersucht. Hier zeigt sich eine deutlich geringer ausgeprägte Zeitabhängigkeit. Wirklich deutlich ist diese lediglich bei der höchsten im Experiment verwendeten Temperatur $T=383 \mathrm{~K} \mathrm{zu}$ beobachten. Es wurden auch wieder hier die Plateauwerte ausgewertet und neben $\epsilon_{1 \omega}^{*}$ und $\epsilon_{3 \omega}^{*}$, der Speichermodul $E^{\prime}$ und der Verlustfaktor tan $\delta$ aufgetragen. Die beiden komplexen Dehnungsamplituden bei $1 \omega$ und $3 \omega$ zeigen beide einen zunächst flachen Anstieg mit der Temperatur und dann einen steileren Anstieg für Temperaturen die ca. 20K unterhalb von $\operatorname{Tg}(390 \mathrm{~K})$ liegen. Dieser steilere Anstieg ist folglich als der Onset des Glasüberganges zu deuten. Für den Speichermodul zeigt sich im Gegensatz zu der Messung am $P M M A^{E}$ für das $\mathrm{PMMA}^{G}$ ein geringer Abfall von $2700 \mathrm{MPa}$ auf $1900 \mathrm{MPa}$ im untersuchten Temperaturbereich. Dies schlägt sich auch im Verlauf des Verlustes nieder, der nur einen geringen Anstieg zeigt. Daher zeigen auch die komplexen Dehnungsamplituden in diesem Fall ebenfalls einen weniger starken Anstieg. Das beobachtete Verhalten von Speicher und Verlust entspricht den Erwartungen und ist konsistent mit anderen standardmäßigen Messmethoden.

Der Einfluss der Spannungsamplitude wurde auch für $\mathrm{PMMA}^{G}$ bei einer Temperatur $10 \mathrm{~K}$ unterhalb von $T_{g}$ untersucht. Auch hier wurde ein weniger ausgeprägtes zeitabhängiges Verhalten beobachtet als bei $\mathrm{PMMA}^{E}$. Lediglich für die beiden höchsten Spanungsamplituden konnte eine Zeitabhängigkeit gefunden werden. Die Plateauwerte für die komplexen Dehnungsamplituden zeigen das erwartete Verhalten. Für $\epsilon_{1 \omega}^{*}$ zeigt sich für kleine Spannungen zunächst ein linearer Verlauf, der jedoch bei höheren Spannungen steiler wird. Diese Steigungsänderung ist auf die einsetzende Nichtlinearität zurückzuführen, da die Beiträge höherer Ordnung signifikante Beiträge liefern. Der Verlauf von $\epsilon_{3 \omega}^{*}$ zeigt eine sehr gute Übereinstimmung mit einer kubischen Fit-Funktion und ist damit konsistent mit der Theorie. Auch der Verlauf des Speichermoduls und des Verlustes reproduzieren das erwartete Verhalten. Das Absinken von $E^{\prime}$ wird begleitet von einem simultanen Anstieg des Verlustes.

\subsubsection{Vergleich der Messungen an $\mathrm{PMMA}^{E}$ und $\mathrm{PMMA}^{G}$}

Die nichtlinearen Effekte zeigten sich in den Messungen an $\mathrm{PMMA}^{E}$ deutlicher als beim $\mathrm{PMMA}^{G}$. Dies ist darauf zurückzuführen, dass der Glasübergang bei PMMA ${ }^{E}$ breiter ist und die hier vorgestellten Messungen in einem Temperaturbereich durchgeführt wurden, der schon im Onset des Glasüberganges liegt. Dadurch wurden für 
die angelegten Spannungen plastische Verformungen hervorgerufen, die dazu führen können, dass sich die Probengeometrie stark ändert. Die ermittelten Werte für die Phasenverschiebung von Anregungs- und Antwortsignal zeigte nicht das erwartete Verhalten. Im Gegensatz dazu konnten am $\mathrm{PMMA}^{G}$ die aus der Theorie erwarteten Abhängigkeiten von der externen Spannungsamplitude gefunden werden. Daher wurden diese Messungen herangezogen um daraus die Nachgiebigkeiten 3.Ordnung $\left(C_{1}^{3}, C_{3}^{3}\right) \mathrm{zu}$ bestimmen. Beide Größen zeigen die erwartete Proportionalität zu $\sigma_{0}^{2}$. Dabei ist jedoch $C_{3}^{3}$ experimentell leichter zugänglich und zeigt direkt die Nachgiebigkeit bei der 3.Harmonischen. Die Größe $C_{1}^{3}$ hingegen wurde als $\Delta C$ bestimmt, wozu es nötig ist, jeweils auch die lineare Nachgiebigkeit bei der fundamentalen Frequenz zu bestimmen. Der Zusammenhang zwischen $C_{1}^{3}$ und $C_{3}^{3}$ bietet ein interessantes Feld für weiterführende Messungen.

\subsubsection{Relaxationszeiten}

Um die Zeitabhängigkeit zu untersuchen, sollen Zeitkonstanten aus den einzelnen Messungen bestimmt werden. Da angenommen wird, dass es sich bei der beobachteten Zeitabhängigkeit um die Signatur des $\alpha$-Prozesses handelt, wird erwartet, dass die beobachtet Zeitabhängigkeit einerm Kohlrausch-William-Watts-Verhalten entspricht. Daher wurde jeweils eine KWW-Funktion mit einem konstanten Streckfaktor $\beta$ an die Kurven angepasst und daraus die Relaxationszeit $\tau_{K W W}$ bestimmt. Dabei ergaben sich jedoch einige Probleme. Einerseits wurden die Messungen nicht lange genug ausgeführt, da am Ende des Hochfeldbereiches immer noch keine Sättigung zu beoabachten ist. Der Plateauwert hat jedoch maßgeblichen Einfluss auf den Fitprozess. Desweitern sind alle Messungen von einer durch die Temperaturregelung hervorgerufenen Schwingung überlagert, wie in dem Abschnitt 3.3.6 dargestellt wurde. Daher können die hier ermittleten Werte für $\tau_{K W W}$ lediglich als ein Trend verstanden werden und insbesondere die absoluten Werte sind nicht verläßlich. Trotzdem sollen im Folgenden die ermittelten Trends für die Zeitkonstante $\tau_{K W W}$ des nichtlinearen Effektes diskutiert werden. Die Messungen an PMMA ${ }^{E}$ zeigten jeweils eindeutig eine Zeitabhängigkeit. In Abhängigkeit der Temperatur kann dabei der folgende Trend beobachtet werden. Mit steigender Temperatur zeigt die Relaxationszeit zunächst einen Anstieg um dann ab $373 \mathrm{~K}$ abzufallen. Nach der Theorie wird ein VFT-Verhalten bei Annäherung an $T_{g}$ erwartet. Der zunächst beobachtete Anstieg kann darauf zurückgeführt werden, dass unterhalb von $T_{g}$ schon kleinere Relaxationsprozesse möglich sind. Im Bild der PEL kann das bedeuten, dass die Temperatur schon ausreichend ist, dass das System eine lokale Energiebarriere überwinden kann. Sobald die Temperatur erhöht wird, können weitere Barrieren überwunden werden. In Abhängigkeit der Spannungsamplitude zeigt sich kein systematisches Bild und die beobachteten Relaxationszeiten streuen stark um einen Wert von ca. 150 s. Für tiefergehende Interpretationen ist die Datenlage nicht ausreichend. Insgesamt kann festgestellt werden, dass die Relaxationszeiten, der Fundamentalen und der dritten harmonischen Dehnungsamplitude ein übereinstimmendes Verhalten zeigen. Für das 
PMMA $^{G}$ wird nur das Ansteigen der Relaxationszeit mit steigender Temperatur beobachtet. Dies kann darauf zurückgeführt werden, dass sich das Material noch weiter vom Onset des Glasbüberganges befindet. Es sind lokale Prozesse möglich. Sobald auch die Energie ausreicht um kooperativere Prozesse stattfinden zu lassen, steigt die Relaxationszeit. In Abhängigkeit der Spannungsamplitude kann ein Absinken der Relaxationszeit beobachtet werden. Für direkte Aussagen sind jedoch weitere Messungen nötig, da in diesem Fall nur 3 Datenpunkte vorliegen. 



\section{UV-aktive Blockcopolymere}

In diesem Teil der Arbeit werden die Ergebnisse der Untersuchungen an dem Blockcopolymersystem $\mathrm{P}(\mathrm{MMA}-c o-\mathrm{CMA})-b$-PBA vorgestellt. Als erstes wird die thermische Stabilität anhand thermogravimetrischer Messungen überprüft. Anschließend werden die Temperaturscans mit dem DMA gezeigt. Zunächst werden dabei die Messungen der einzelnen Teilkomponenten PBA, PMMA, CMA des Blockcopolymers beschrieben. Dann werden die Untersuchungen des Methylmethacrylatblockes mit eingebauten Coumarineinheiten $\mathrm{P}(\mathrm{MMA}-\mathrm{co}-\mathrm{CMA})$ dargestellt. Abschließend werden die Temperaturscans für verschiedene Zusammensetzungen des kompletten Blockcopolymers behandelt.

\subsection{Thermische Stabilität}

Um die thermische Stabilität der Blockcopolymere zu untersuchen, wurden diese thermogravimetrisch analysiert. In Abbildung 5.1 sind für jeweils vier verschiedene Zusammensetzungen TGA-Messungen dargestellt. Es zeigt sich, dass das Probengewicht bis zu einer Temperatur von $623 \mathrm{~K}$ konstant bleibt und erst bei höheren Temperaturen die Zersetzung des Materials einsetzt. Zu berücksichtigen ist, dass die TGA- Messungen unter Argonatmosphäre durchgeführt wurden. Andere Experimente wurden jedoch unter Luft durchgeführt, so dass hier eine Zersetzung schon bei etwas kleineren Temperaturen auftreten kann. Da jedoch der Temperaturbereich, der in den Experimenten genutzt wird sich bis maximal $523 \mathrm{~K}$ erstreckt, also etwa $373 \mathrm{~K}$ unterhalb der Temperatur, bei der die Zersetzung einsetzt, kann davon ausgegangen werden, dass die beobachteten Effekte nicht auf eine Degradierung des Materials zurückzuführen sind. Neben dem Probengewicht ist in Abbildung 5.1 ebenfalls das DTA-Signal, sowie die jeweilige Basislinie eingetragen. Die DTA-Signale sinken jeweils mit der Temperatur und zeigen dabei zusätzlich noch einige kleinere Stufen. Bei etwa $603 \mathrm{~K}$ zeigt sich ein ausgeprägtes Minimum, an welches sich ein deutliches Maximum bei der Degradationstemperatur anschließst. Ein Minimum im DTA-Signal bedeutet, dass endotherme Prozesse im Probenmaterial stattfinden. 


\section{UV-aktive Blockcopolymere}
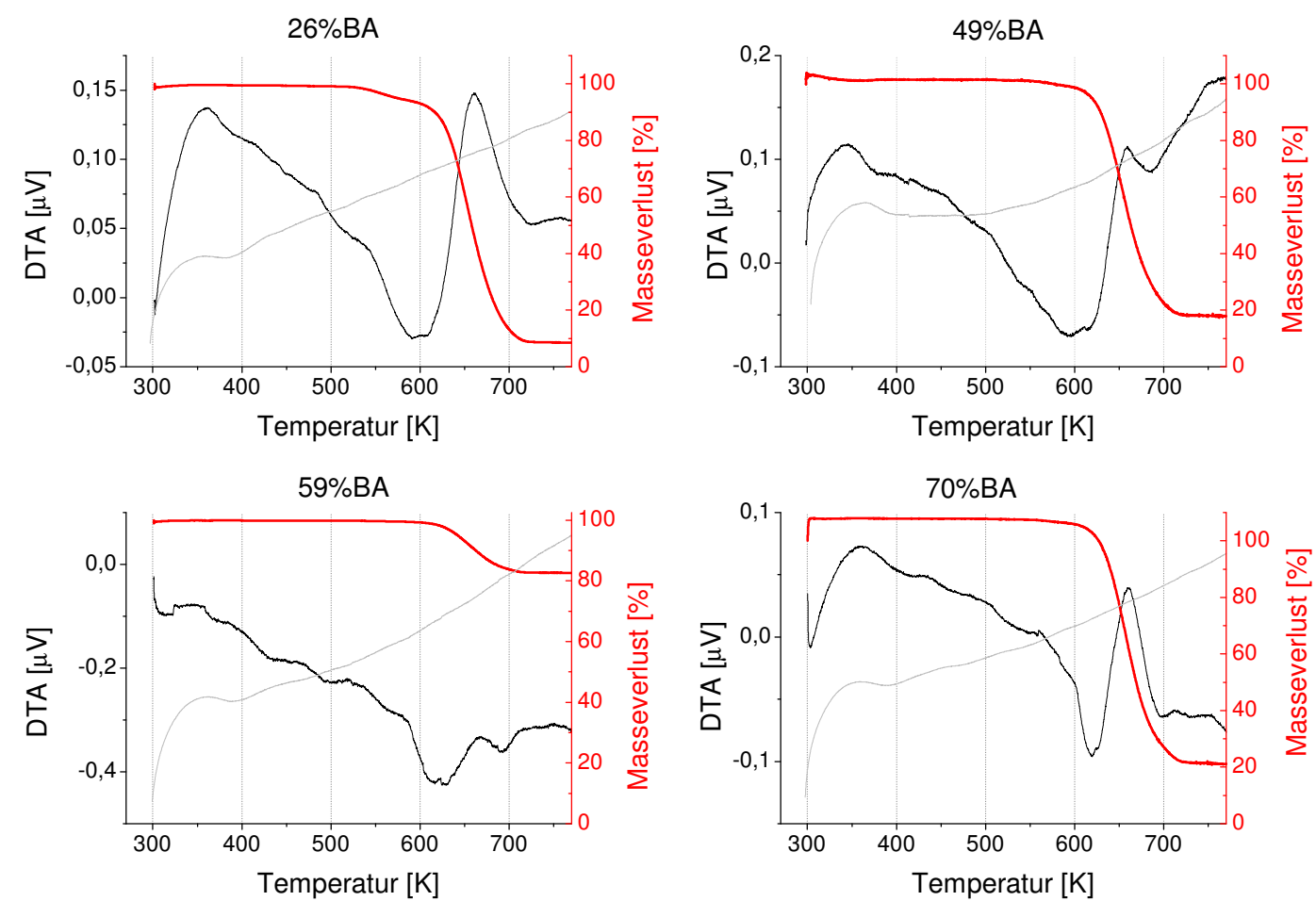

Abb. 5.1.: Thermogramme für BCP-Proben mit verschiednem BA-Anteil. Es sind Massenverlust (rot) DTA-Signal (schwarz) und Basislinie des DTA-Signals (grau) eingezeichnet. 


\subsection{Temperaturabhängigkeit der mechanischen Eigenschaften}

Die Blockcopolymerproben wurden hinsichtlich der Temperaturabhängigkeit der mechanischen Eigenschaften untersucht, indem sie mithilfe von Material Pockets in einem Biegebalkenversuch in dem DMA gemessen wurden. Die Temperatur wurde mit einer Rate von $2 \mathrm{~K} / \mathrm{min}$ von $193 \mathrm{~K}$ auf $523 \mathrm{~K}$ erhöht und dabei mit einer Auslenkung von $0,02 \mathrm{~mm}$ und einer Frequenz von $5 \mathrm{~Hz}$ gemessen. Es wurden zunächst die einzelnen Komponenten, aus denen das Blockcopolymersystem besteht, untersucht. Dabei sind die Homopolymere ebenso wie die später analysierten Blockcopolymere mithilfe der RAFT-Polymerisationsmethode von K.Tietz hergestellt worden, um eine Vergleichbarkeit zu gewährleisten. Es wurden auch das Coumarin, sowie verschiedene MMA-Blöcke in welche das Coumarin schon integriert wurde, untersucht.

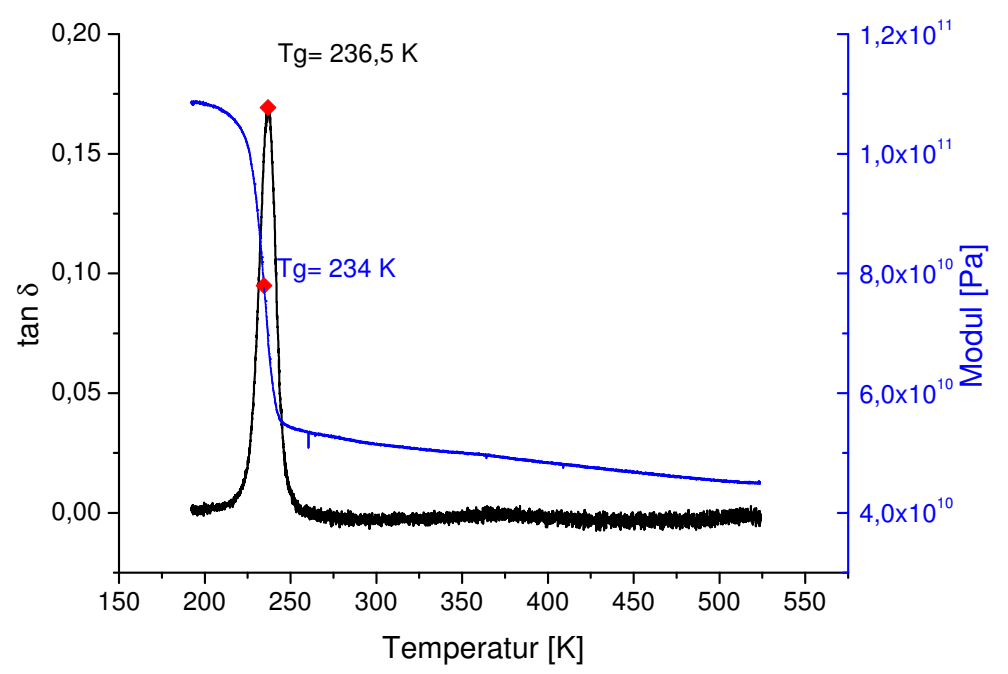

Abb. 5.2.: Speichermodul und $\tan (\delta)$ des reinen Butylacrylates in Abhängigkeit der Temperatur, gemessen während des Aufheizens. Die bestimmten Glastemperaturen sind in rot eingezeichnet.

\subsubsection{Einzelne Komponenten der BCPs}

Für das reine Polybutylacrylat ist in Abbildung 5.2 die Temperaturabhängigkeit des Modul und des $\tan \delta$ aufgetragen. Es wurden jeweils die Glastemperaturen nach den in Kapitel 3.3.4 beschriebenen Methoden ermittelt, sie sind in der Abbildung mit roten Punkten gekennzeichnet. Im Verlauf des Speichermoduls zeigt sich eine deutliche Stufe bei $234 \mathrm{~K}$ und es nimmt der Speichermodul wie erwartet mit steigender Temperatur ab. Der scharfe Peak im $\tan \delta$ liefert eine Glasübergangstemperatur 


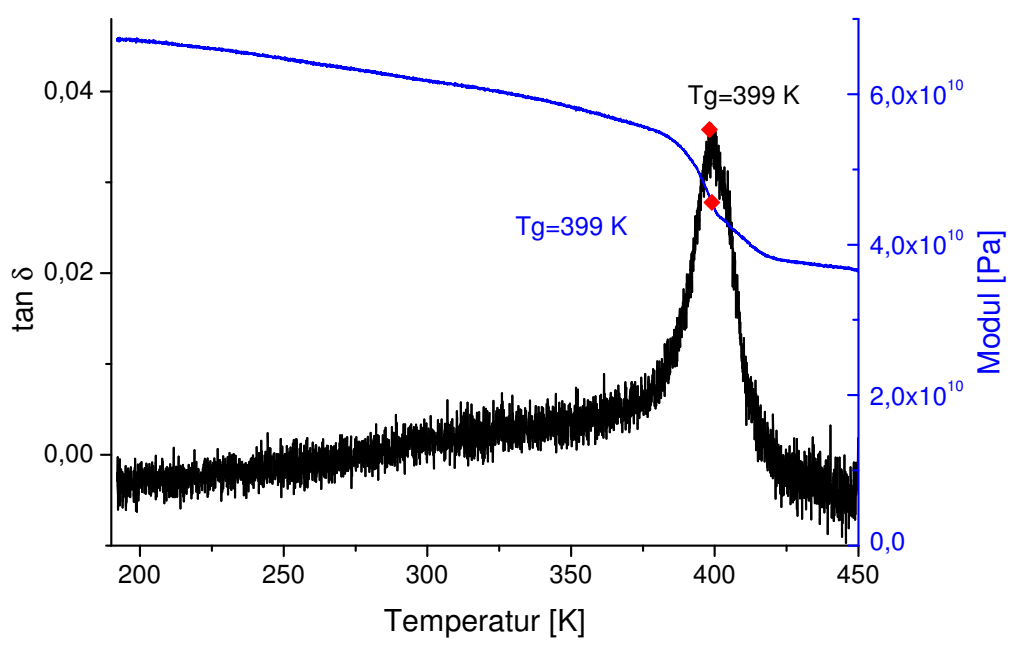

Abb. 5.3.: Temperaturabhängigkeit des Modul und des $\tan (\delta)$ von RAFT synthetisiertem PMMA.

von $T_{g}=236,5 \mathrm{~K}$. In der Literatur ist die Glasübergangstemperatur von Polybutylacrylat mit einer Temperatur von $233 \mathrm{~K}$ angegeben [14] und damit in guter Übereinstimmung mit dem hier ermittelten Wert.

Die Abbildung 5.3 zeigt den Temperaturscan für das reine mit RAFT synthetisierte Polymethylmethacrylat. Auch hier kann einerseits ein deutlich ausgeprägter $\alpha$-Peak im $\tan \delta$, sowie eine Stufe im Modul bei $T_{g}$ beobachtet werden. Typische Glastemperaturen von PMMA sind in der Literatur in einem Bereich von $373 \mathrm{~K}-393 \mathrm{~K}$ angegeben.[14] Die relativ große Streuung der Literaturwerte ist auf den Einfluss des Herstellungsverfahrens zurückzuführen.

In den PMMA-Block der nachfolgend untersuchten Blockcopolymere sind CoumarinEinheiten statistisch eingebaut. Daher wirde hier zunächst der Einfluss des Coumarins auf den MMA-Block und sein temperaturabhängiges mechanisches Verhalten untersucht. Das Coumarin ist eine einfache organische Verbindung mit der Summenformel $\mathrm{C}_{9} \mathrm{H}_{6} \mathrm{O}_{2}$ und liegt bei Raumtemperatur als kristallines Pulver vor. Aus dem Temperaturscan mit dem DMA konnte eine Schmelztemperatur von $T_{m}=345 \mathrm{~K}$ bestimmt werden. Dies ist in guter Übereinstimmung mit dem Literaturwert von $T_{m}=343 \mathrm{~K}$ aus [101]. Die Messung ist in Abbildung A.3 im Anhang zu finden.

Das Coumarin wird während der Polymerisation statistisch in die PMMA-Blöcke eingebaut, so dass zunächst $\mathrm{P}(\mathrm{MMA}-\mathrm{co}-\mathrm{CMA})$ Copolymere erhalten werden. In der Abbildung 5.4 sind für drei verschiedene $\mathrm{P}(\mathrm{MMA}-$-co-CMA) Blöcke der mit dem DMA gemessene temperaturabhängige $\tan \delta$ aufgetragen. Das Coumarin führt zu einer Verschiebung der Glasübergangstemperatur des reinen PMMA $\left(T_{g}=399 \mathrm{~K}\right) \mathrm{zu}$ niedrigeren Temperaturen. In Hinblick auf die unterhalb der Glasübergangstemperatur von PMMA liegenden Schmelztemperatur des Coumarins ist dies konsistent. Die 


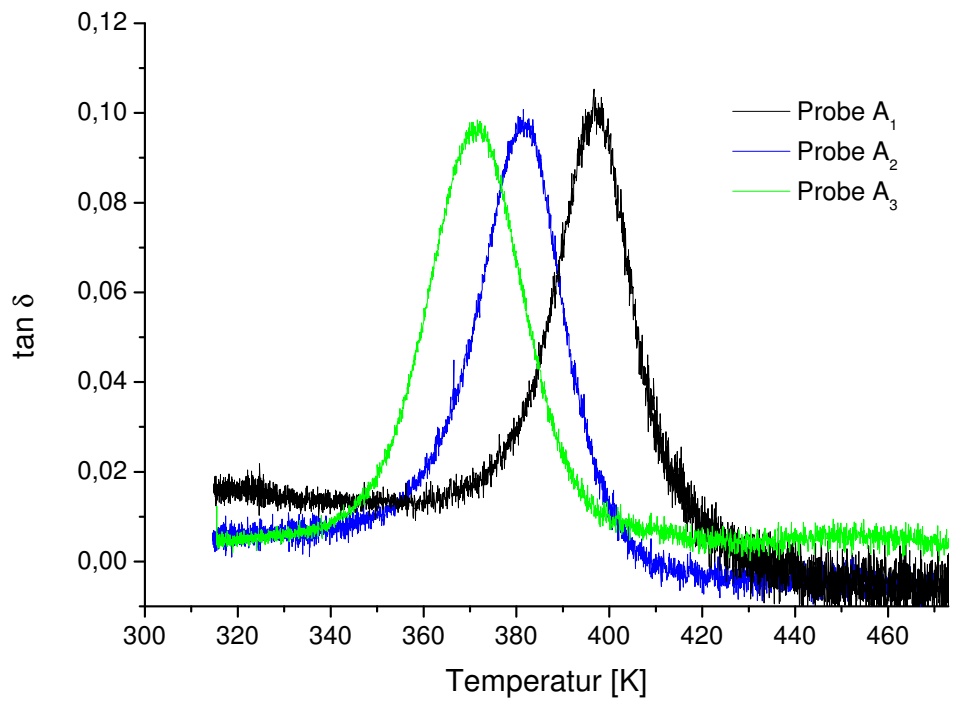

Abb. 5.4.: Peak im tan $\delta$ gemessen während des Abkühlens für die PMMA-co-CMA-Blöcke.

\begin{tabular}{lrcr} 
Probe & $M_{n}[\mathrm{~g} / \mathrm{mol}]$ & $D$ & $T_{g}[\mathrm{~K}]$ \\
\hline \hline$A_{1}$ IV 35-36 & 47500 & 1,30 & $397 \pm 1$ \\
$A_{2}$ I 28 & 12000 & 1,20 & $387 \pm 1$ \\
$A_{3}$ III 53-4 & 5800 & 1,18 & $363 \pm 1$ \\
\hline
\end{tabular}

Tab. 5.1.: Übersicht der Glasübergangstemperaturen der P(MMA-co-CMA)-Blöcke, sowie deren Molgewichte $M_{n}$ und Dispersitätsindizes $D$

Tabelle 5.1 zeigt für einige der Blöcke $\mathrm{P}(\mathrm{MMA}-$-co-CMA) die aus den mit dem DMA gemessenen Temperaturscans ermittelten Glastemperaturen, sowie Molgewichte und Dispersitätsindizes.

Für die P(MMA-co-CMA) Blöcke wird beobachtet, dass die Glasübergangstemperatur stark mit dem Molekulargewicht, also der Kettenlänge abnimmt. Zusätzlich steigt auch die Polydispersität mit zunehmendem Molgewicht. Die erhöhte Dispersität kann auf den Herstellungsprozess zurückgeführt werden. Offensichtlich finden während der Polymerisation Abbruchreaktionen statt, die dazu führen, dass die Polydispersität für höhere Molgewichte zunimmt.

\subsubsection{Blockcopolymere}

Die Blockcopolymere P(MMA-co-CMA)- $b$-PBA zeigen in den Temperaturscans jeweils zwei Glasübergänge. Eine Übersicht der ermittelten Glasübergangstemperaturen ist in der Tabelle 5.2 dargestellt. Der Glasübergang des BA-Anteiles ist für alle Zusammensetzungen nahezu unverändert und lediglich im Vergleich zum reinen 
Butylacrylate etwas zu höheren Temperaturen verschoben. Im Vergleich dazu ist auffällig, dass die Glasübergangstemperatur von PMMA mit steigendem ButylacrylatAnteil stark abnimmt.

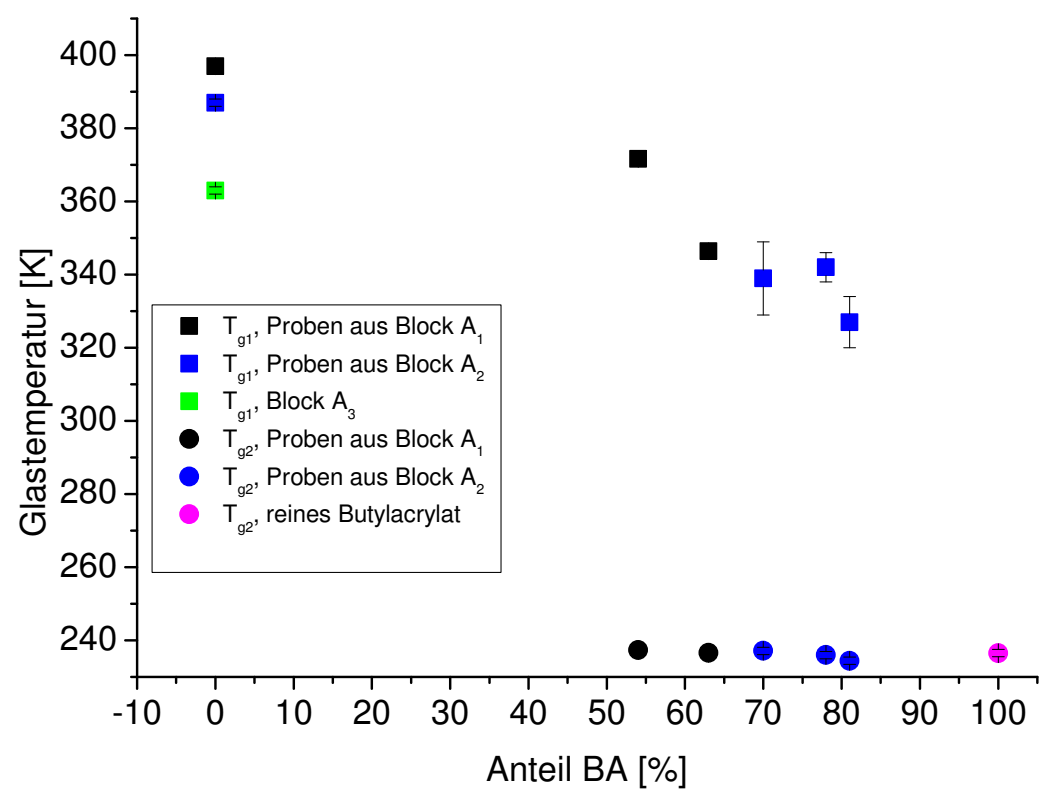

Abb. 5.5.: Übersicht der Glasübergangstemperaturen der untersuchten Blockcopoymere

\begin{tabular}{llllll} 
Probe & $M_{n}[\mathrm{~g} / \mathrm{mol}]$ & $D$ & $\% \mathrm{BA}$ & $T_{g 1}[K]$ & $T_{g 2}[K]$ \\
\hline \hline D 37-08 & 00 & 0 & 52 & 372 & 237 \\
E 37-10 & 00 & 0 & 61 & 346 & 237 \\
F 30-05 & 00 & 0 & 68 & 339 & 237 \\
G 47-01 & 00 & 0 & 75 & 342 & 235 \\
H 47-03 & 00 & 0 & 81 & 327 & 234 \\
\hline
\end{tabular}

Tab. 5.2.: Übersicht der Glasübergangstemperaturen der untersuchten Blockcopolymere, sowie deren Molgewichte $M_{n}$ und Dispersionsindizes D.

Im Folgenden werden die einzelnen DMA-Messungen näher untersucht und sollen im Diskussionsteil mit den aus [93] vorliegenden AFM-Messungen verglichen werden.

Die Abbildung $\underline{5.6}$ zeigt die Temperaturabhängigkeit des Moduls und des $\tan \delta$ der Probe D (BA-Gehalt 52\%). Bei tiefen Temperaturen zeigt sich ein scharfer Peak mit einer Peakposition von $T_{g 2}=237 \mathrm{~K}$. Zusätzlich ist ein breites Maximum zwischen $323 \mathrm{~K}$ und $453 \mathrm{~K}$ zu sehen, bei dem es sich auch um die Überlagerung zweier breiter Maxima handeln kann. Der Modul sinkt mit der Temperatur und zeigt eine deutliche Stufe bei $T_{g 2}$, was mit dem Erweichen des BA-Blockes in Zusammenhang steht. Ab $273 \mathrm{~K}$ sinkt der Modul kontinuierlich und zeigt eine breite Stufe zwischen $323 \mathrm{~K}$ und 


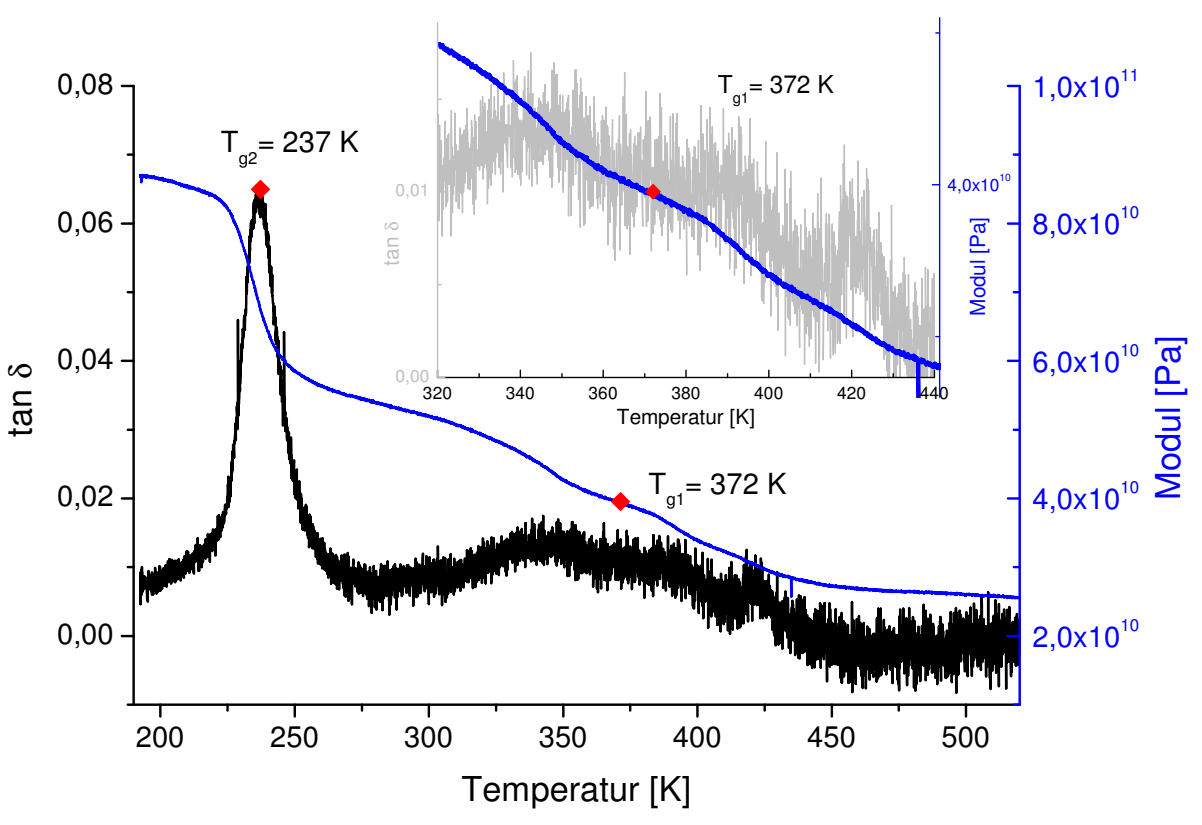

Abb. 5.6.: Speichermodul und $\tan \delta$ der Probe D(37-08). Diese Probe zeigte bei AFM Messungen die Struktur von perforierten Lamellen [93].

$453 \mathrm{~K}$. Ähnlich wie im Verlauf des $\tan \delta$ kann es sich auch hier um die überlagerte Signatur zweier Übergänge handeln. Die Glastemperatur für den P(MMA-co-CMA)Block wurde jedoch unter der Annahme, dass es sich um eine einzelne Stufe handelt bestimmt und gibt eine Temperatur von $T_{g 1}=372 \mathrm{~K}$.

Die Probe E(37-10) hat eine ähnliche Zusammensetzung wie die Probe $\mathrm{D}(37-08)$. Der temperaturabhängige Modul und $\tan \delta$ sind in der Abildung 5.7 dargestellt. Der Verlauf ähnelt sehr dem von der Probe D(37-08). Der Glasübergang der BAKomponente zeigt eine deutliche Stufe im Modul und einen eindeutigen Peak im $\tan \delta$ mit einer Peakposition von $T_{g 1}=237 \mathrm{~K}$. Für höhere Temperaturen nimmt der Modul in einer breiten Stufe ab, die jedoch auch hier eine Überlagerung mehrer Übergänge sein könnte, da zwei weitere sehr schwach Stufen bei $373 \mathrm{~K}$ und $388 \mathrm{~K}$ auftreten. Für Temperaturen oberhalb von $273 \mathrm{~K}$ zeigt sich im $\tan \delta$ jedoch kein echter Peak, sondern vielmehr eine absinkende Flanke.

Der Temperaturscan der Probe $\mathrm{F}(30-05)$ ist in der Abbildung 5.8 gezeigt. Auch für diese Probe kann der Glasübergang des Butylacrylat-Blockes eindeutig aus dem tan $\delta$ bestimmt werden und die dazugehörige Stufe im Modul ist auch deutlich ausgeprägt. Jedoch beginnt für diese Probe der Einfluss der zweiten Erweichungstemperatur schon bei Temperaturen unterhalb von $273 \mathrm{~K}$, so dass die beiden Übergänge ein wenig überlagert sind. Im Vergleich zur Probe E ist auch hier das Maximum im $\tan \delta$ vielmehr eine abfallende Flanke.

Für die Probe G(47-01) mit 75\% BA-Anteil ist der Temperaturscan mit dem DMA in Abbildung 5.9 dargestellt. Der $\tan \delta$ dieser Probe zeigt einen deutlichen Peak bei 


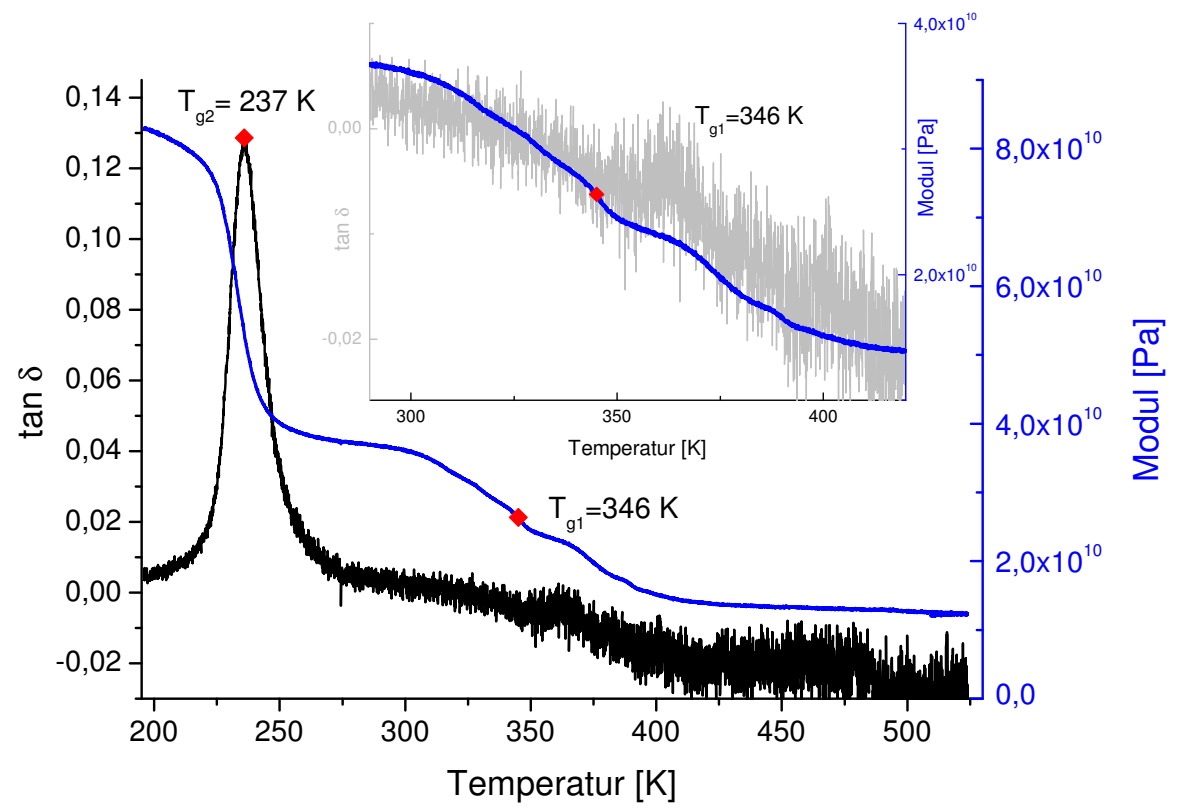

Abb. 5.7.: Speichermodul und $\tan \delta$ der Probe E(37-10). Diese Probe zeigte bei AFMMessungen die Struktur von perforierten Lamellen [93].

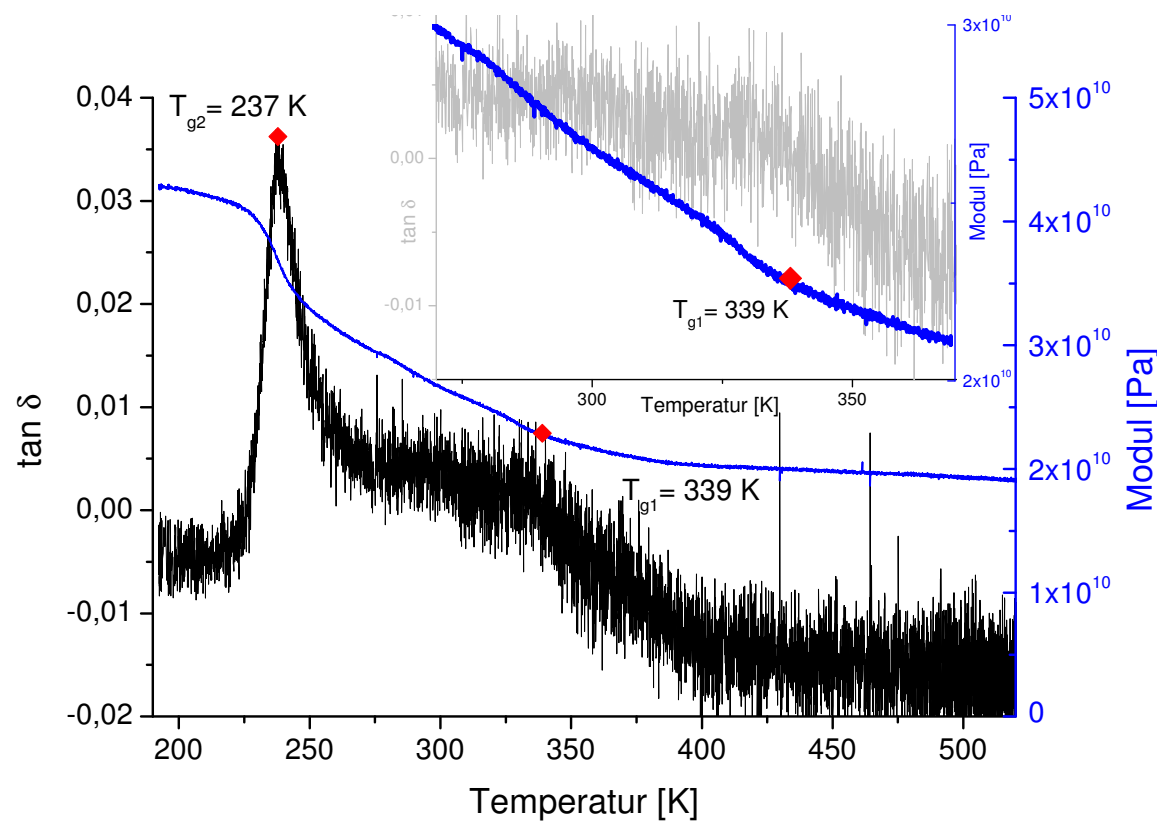

Abb. 5.8.: Speichermodul und $\tan \delta$ der Probe F(30-05). Diese Probe zeigte bei AFM Messungen die Struktur von liegenden PMMA Zylindern [93]. 


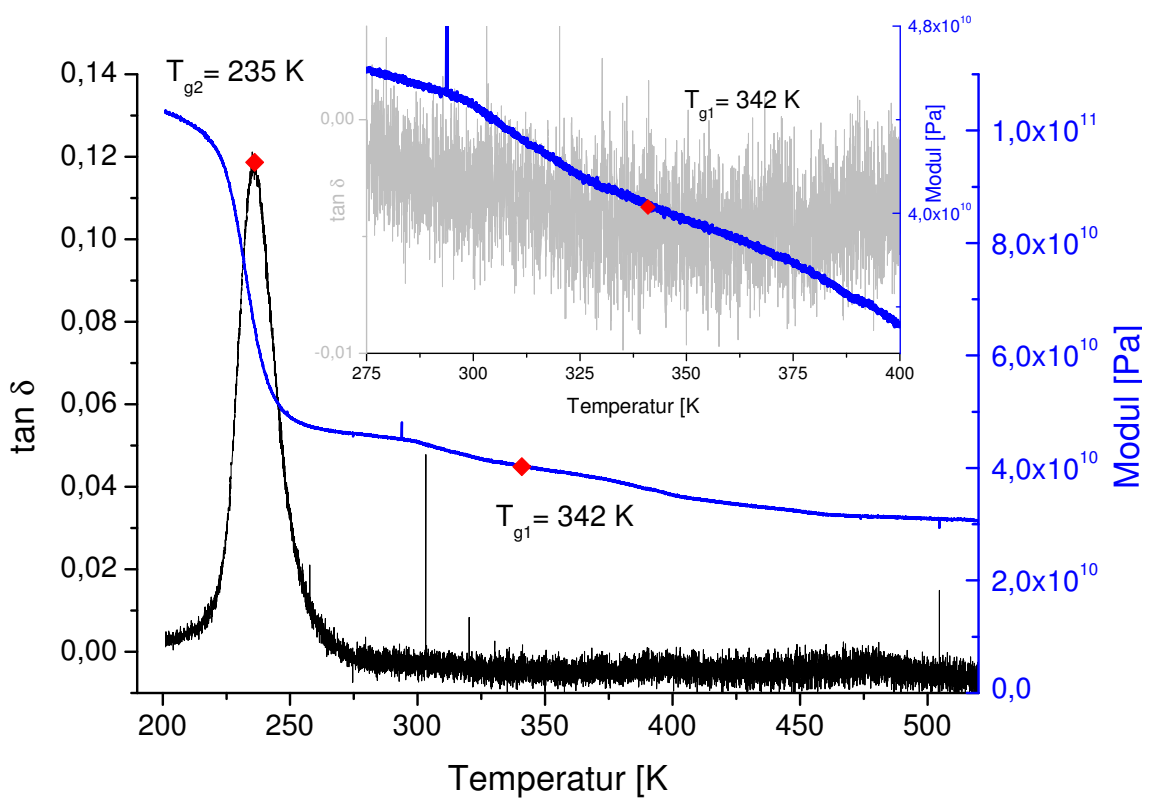

Abb. 5.9.: Speichermodul und $\tan \delta$ der Probe G(47-01). Diese Probe zeigte im AFM die Struktur von hexagonal geordneten Sphären [93].

einer Temperatur von $235 \mathrm{~K}$, der Erweichungstemperatur des BA-Anteiles der Probe. Weitere Maxima sind im Verlauf nicht zu beobachten. Der Speichermodul der Probe zeigt ebenfalls deutlich die Erweichung des BA-Anteiles anhand einer deutlichen Stufe. Eine weitere Stufe kann für höhere Tenperaturen beobachtet werden, erstreckt sich jedoch über einen großen Temperaturbereich von etwa $298 \mathrm{~K}$ bis hin zu $313 \mathrm{~K}$ und ist sehr schwach ausgerägt. Die bestimmte Glasübergangstemperatur liegt bei $342 \mathrm{~K}$.

Die Probe mit dem höchsten BA-Anteil von $81 \%$ ist die Probe H(47-03). Der temperaturabhängige Speichermodul und $\tan \delta$ sind in der Abbildung $\underline{5.10}$ dargestellt. Wieder zeigen sowohl Speichermodul, als auch $\tan \delta$ deutlich den Glasübergang des BA-Blockes. Der weitere Verlauf des $\tan \delta$ zeigt zudem noch ein relativ breites Maximum zwischen $273 \mathrm{~K}$ und 323K. Der Speichermodul zeigt zudem in diesem Bereich eine breite Stufe, da hier das Erweichen des P(MMA-co-CMA) Blockes stattfindet. Die aus der Tangentenmethode bestimmte Temperatur liegt bei 327K. 


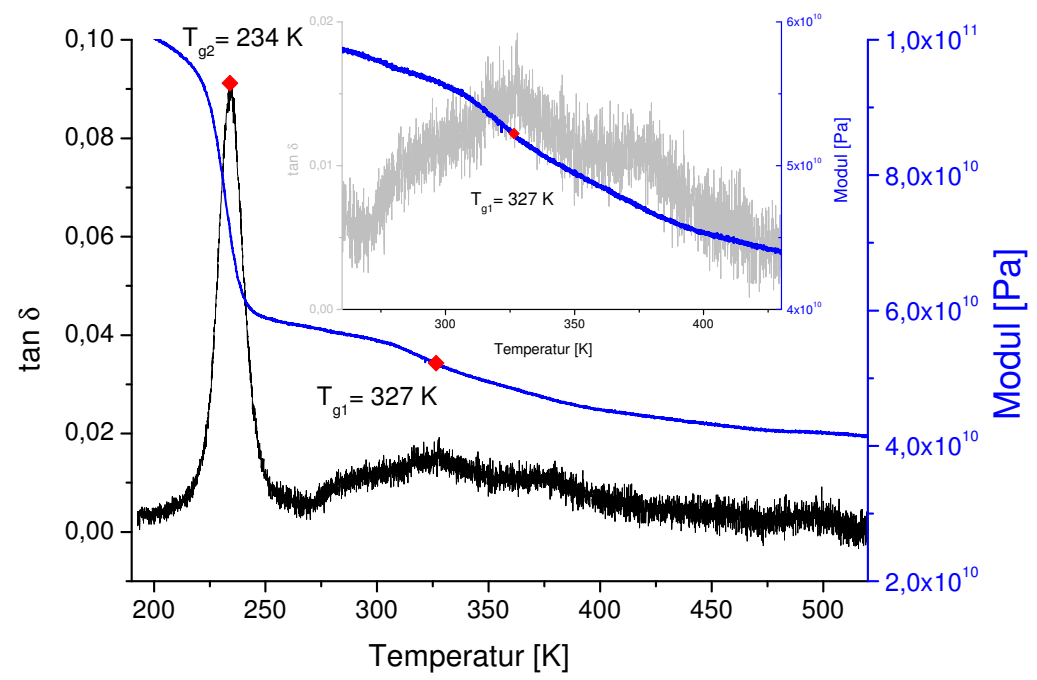

Abb. 5.10.: Speichermodul und $\tan \delta$ der Probe H47-03). Diese Probe zeigte im AFM die Struktur von hexagonal geordneten Sphären [93].

\subsection{Kleinwinkelröntgenstreuung}

Es wurden zwei verschieden Probenchargen untersucht. Neben den P(MMA-coCMA)- $b$-PBA Proben wurden zusätzlich PMMA-co-PBA Proben untersucht, also Blockcopolymere ohne integriertes Coumarin. Da jedoch das Coumarin keinen signifikanten Einfluss auf das Verhalten der Blockcopolymere gezeigt hat, können diese Daten mit denen der coumarinhaltigen Proben verglichen werden. Trotzdem werden jedoch nur Proben mit einem BA-Gehalt $\leq 80 \%$ betrachtet um einen eventuellen Einfluss des Coumarins gering zu halten.

Anhand der folgenden Messungen soll überprüft werden, ob die im AFM beobachtete Mikrophasenseparation nicht nur ein Oberflächeneffekt, sondern auch im Bulk vorhanden ist. Eine charakteristische Längenskala wurde jeweils aus dem dominantesten Peak ermittelt und wird im Diskussionsteil mit denen aus den AFM-Bildern verglichen.

In Abbildung $\underline{5.11}$ sind die Röntgenspektren für einige der Proben gezeigt.

Dabei werden verschiedene Kategorien von Verhalten beobachtet. Die Proben mit einem geringen Gehalt des BA-Monomers $\left(f_{B A}<50 \%\right)$ zeigen ein sehr ähnliches Verhalten. Es wird lediglich ein einzelner sehr breiter Peak bei ca. $q=0.0451 \AA^{-1}$ was einer Länge von ca. $14 \mathrm{~nm}$ entspricht beobachtet.

Für Proben mit einem BA-Gehalt zwischen $89 \%$ und $80 \%$ zeigt sich zusätzlich zu dem Peak bei $14 \mathrm{~nm}$ ein gut augeprägter Peak bei kleineren q-Werten $(q \approx$ $\left.0.021 \AA^{-1}\right)$. Dies bedeutet eine Ordnung auf einer Längenskala von 30nm. Die Probe mit der Zusammensetzung von einem BA- Gehalt von 63\% (37-10) zeigt in den 

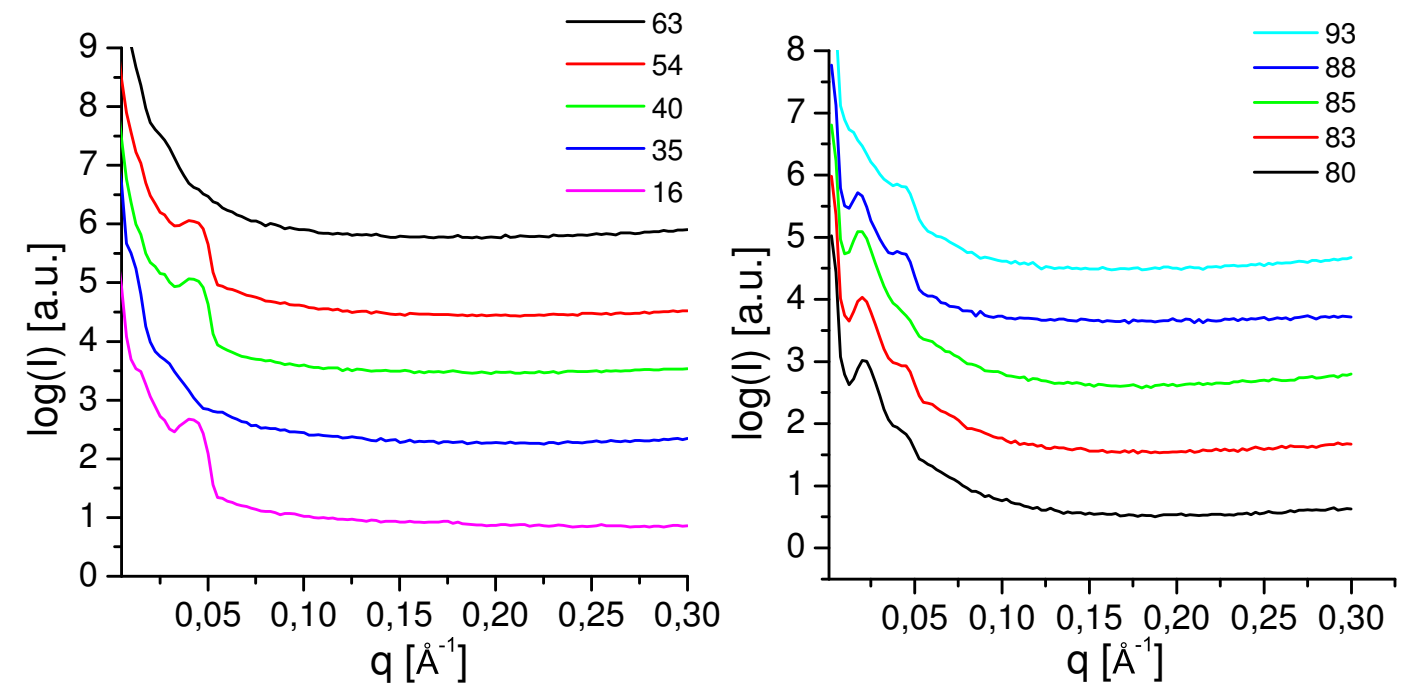

Abb. 5.11.: Röntgen-Spektren, für Proben mit verschiedenem BA-Gehalt. Es ist der Logarithmus der gemessenen Intensität in Abhängigkeit des Streuvektors q aufgetragen.

SAXS-Messungen keinerlei Peaks. Für einen etwas geringeren BA-Gehalt (54\% 3708) taucht wieder ein Peak im Spektrum auf. Dieser ist verknüpft mit einer charakteristischen Längenskala von ca. $14 \mathrm{~nm}$ und weist daraufhin, dass die einzelnen Blöcke eine Nahordnung mit einer typischen Domänengröße von 14nm haben. Auf eine Fernordnung gibt es keinerlei Hinweise aus dem Spektrum. In der Tabelle $\underline{5.3}$ sind für die Proben die ermittelten charakteristischen Längen zusammengefasst

\begin{tabular}{lcccc} 
Probe & BA-Anteil & $d_{1}$ & $d_{2}$ & \\
\hline \hline $37-03$ & 16 & $14 \mathrm{~nm}$ & - & mit CMA \\
$37-05$ & 35 & $14 \mathrm{~nm}$ & - & mit CMA \\
$37-06$ & 40 & $14 \mathrm{~nm}$ & - & mit CMA \\
D $37-08$ & 54 & $14 \mathrm{~nm}$ & - & mit CMA \\
E $37-10$ & 63 & - & - & mit CMA \\
$10-09$ & 85 & - & $30 \mathrm{~nm}$ & ohne CMA \\
$10-02$ & 89 & $14 \mathrm{~nm}$ & $30 \mathrm{~nm}$ & ohne CMA \\
$10-04$ & 93 & $14 \mathrm{~nm}$ & - & ohne CMA \\
\hline
\end{tabular}

Tab. 5.3.: Übersicht der charakteristischen Längen der untersuchten Blockcopolymere, bestimmt aus Kleinwinkelröntgenstreuung. 


\subsection{Lichtinduziertes Crosslinking und Decrosslinking}

Der Einfluss von strukturellen Einschränkungen auf Mikrophasenseparation von Blockcopolymeren kann experimentell untersucht werden, indem Vernetzungen in das Polymer gebracht werden. Eine Möglichkeit der Vernetzung bieten photoaktive Monomere. In dem untersuchten Polymersystem wurde in den MMA-Block ein photoaktives Coumarinderivat statistisch eingebaut. Bei Bestrahlung mit UV-Licht der geeigneten Wellenlänge $\lambda>310 \mathrm{~nm}$ können sich zwischen den Coumarineinheiten Dimerbindungen bilden. Für die hier untersuchten Proben würde dies zu einer Vernetzung der P(MMA-co-CMA) Blöcke untereinander führen. Besonders interessant ist, dass die Dimerisierungsreaktion reversibel ist und durch UV-Licht der Wellenlänge $\lambda=254 \mathrm{~nm}$ die Crosslinks theoretisch wieder geöffnet werden können. Hervorzuheben ist auch, dass die Vernetzung hier im festen Zustand des Polymers stattfindet. In der Literatur wird bisher jedoch hauptsächlich von Vernetzungsreaktionen in Lösung berichtet [40]. Das ermöglicht während des Vernetzungsprozesses die mechanischen Eigenschaften direkt in situ zu untersuchen. Besonders interessant könnten daraus resultierende Anwendungen sein, da je nach Anspruch an die Materialeigenschaften mit Licht der geeigneten Wellenlänge bestrahlt werden kann, ohne dass das Polymer dafür in Lösung gebracht werden muss. Zudem bietet die Vernetzung im festen Zustand die Möglichkeit Konfigurationen „einzufrieren“. So kann beispielsweise die Morphologie für eine bestimmte Temperatur vernetzt werden und bei anderer Temperatur untersucht werden. Dies wird später im Abschnitt 5.5 für den Nachweis des Ordnungs-Unordnungs-Überganges mit dem AFM genutzt.

Zunächst konnte K.Tietz mithilfe von UV/Vis-Spektrometrie zeigen, dass die Vernetzung tatsächlich stattfindet. Dafür wurde beobachtet, dass die Absorbanz der Coumarindoppelbindung $(\lambda=317 \mathrm{~nm})$ abnimmt, wenn dünne Polymerfilme mit Licht der Wellenlänge $\lambda>310 \mathrm{~nm}$ bestrahlt werden. Vernetzt nämlich die Probe, so bilden sich jeweils Dimer zwischen zwei Coumarineinheiten, so dass weniger Coumarindoppelbindungen im Material vorhanden sind und damit die Absorbanz sinkt. Diese Messung ist in der Abbildung $\underline{5.12}$ dargestellt, es wurde daraus ein Grad der Photodimerisierung von etwa $40 \%$ bestimmt.

Ob die Vernetzungareaktion auch im Bulk stattfindet und wie sie die mechanischen Eigenschaften beeinflusst, wurde zunächst mithilfe von Temperaturscans untersucht. Dafür wurde unbestrahltes Probenmaterial der Probe F(30-5, 70\%BA) mit dem gleiche Temperatuprotokoll wie in Abschnitt 5.2 in einem Materialpocket untersucht. Ein anderer Teil der Probe F(30-5, 70\%BA) wurde mit UV-Licht der Wellenlänge $\lambda<310 \mathrm{~nm}$ bei Raumtemperatur bestrahlt. Für die beiden Temperaturscans ist in der Abbildung 5.13 der tan $\delta$ dargestellt. Beide Proben zeigen einen scharfen Peak bei $234 \mathrm{~K}$, der Erweichungstemperatur des Butylacrylat-Blockes sowohl beim Kühlen von Raumtemperatur zu 193K, als auch beim anschließenden Heizen. 

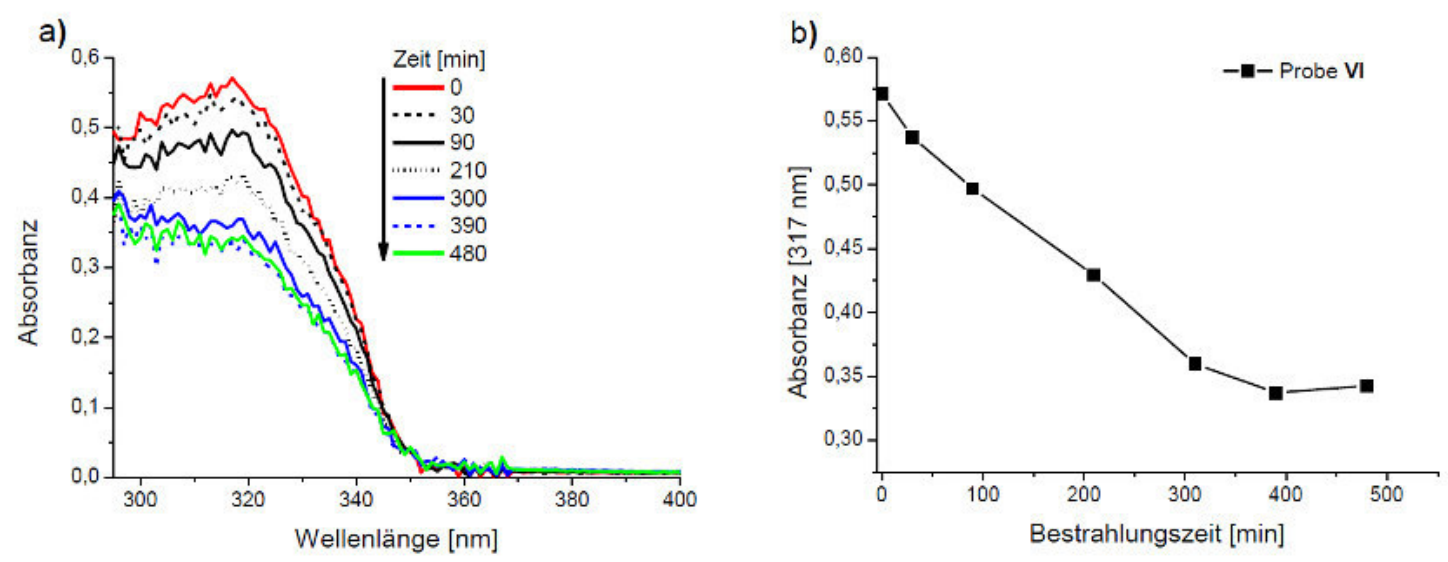

Abb. 5.12.: a)UV-Vis-Spektrum der Probe E(37-10) nach Bestrahlung mit UV-Licht der Wellenlänge $\lambda<310 \mathrm{~nm}$ für verschiedene Bestrahlungsdauern t; b) zeitabhängige Entwicklung der Absorptionsbande bei $317 \mathrm{~nm}$ aus [93].
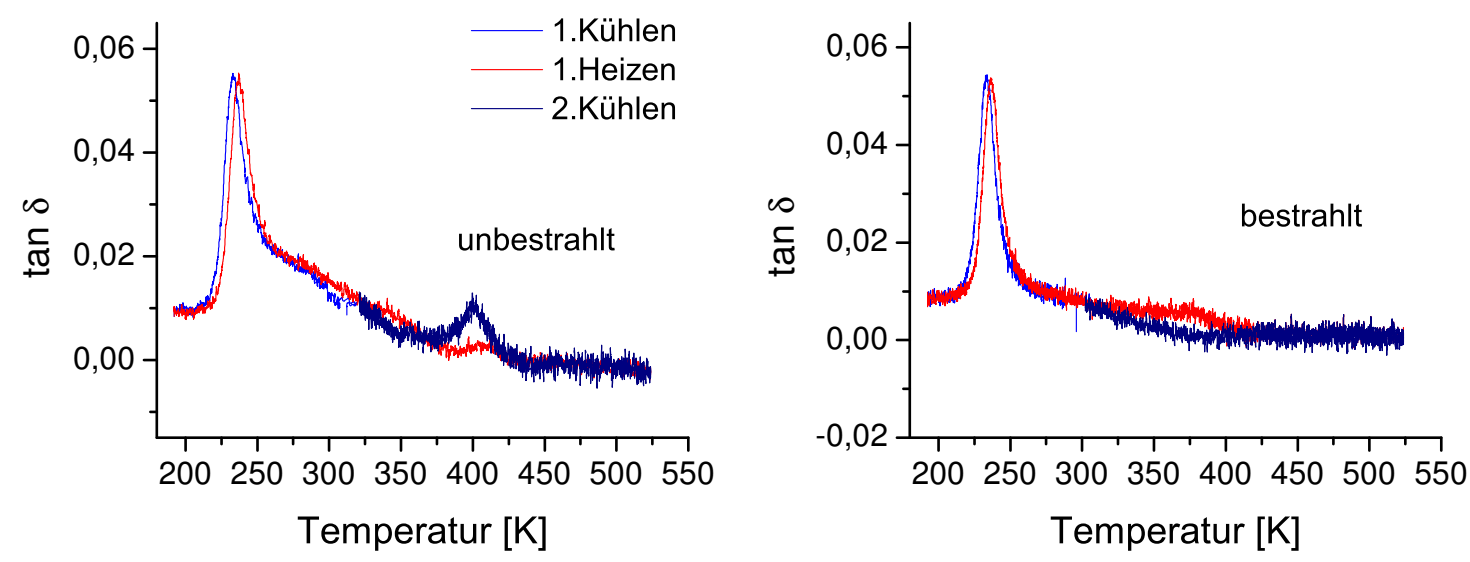

Abb. 5.13.: DMA Temperaturscans einer bestrahlten und einer unbestrahlten Probe F(305) $70 \% B A$. 


\section{UV-aktive Blockcopolymere}

Zudem zeigen beide Proben in der Heizkurve ein schwach ausgeprägtes Maximum im Bereich zwischen 373K und 403K. Beim erneuten Kühlen von 523K zu Raumtemperatur zeigt sich deutlich ein Unterschied zwischen der bestrahlten und der unbestrahlten Probe. Es wird neben dem Glasübergang des BA-Blockes für die unbestrahlte Probe nach dem ersten Heizen eine deutlicher Peak für den Glasübergang des PMMA beobachtet. Das bestrahlte und daher vernetzte Material hingegen kann durch den Vernetzungsprozess nicht mehr relaxieren und die Gleichchgewichtskonfiguration einnehmen. Das dieser Effekt nur den PMMA-Übergang betrifft liegt daran, dass sich die Vernetzungen nur zwischen den PMMA-Blöcken befinden.

Um zu untersuchen, welchen Einfluss die Crosslinks im Material auf die mechanischen Eigenschaften haben, wurde Probenmaterial in Zuggeometrie bei Raumtemperatur dynamisch mechanisch gemessen, während das Material mit UV-Licht der Wellenlänge $\lambda<310 \mathrm{~nm}$ bestrahlt wurde. Für die Probe $\mathrm{D}(37-08)$ ist der Speichermodul in Abhängigkeit der Bestrahlungsdauer in der Abbildung 5.14 abgebildet.Der Verlauf zeigt einen geringen Modulzuwachs von einem Wert von $233 \mathrm{MPa}$ auf einen Wert von $236 \mathrm{MPa}$. Dies entspricht einem Zuwachs von lediglich einem Prozent.

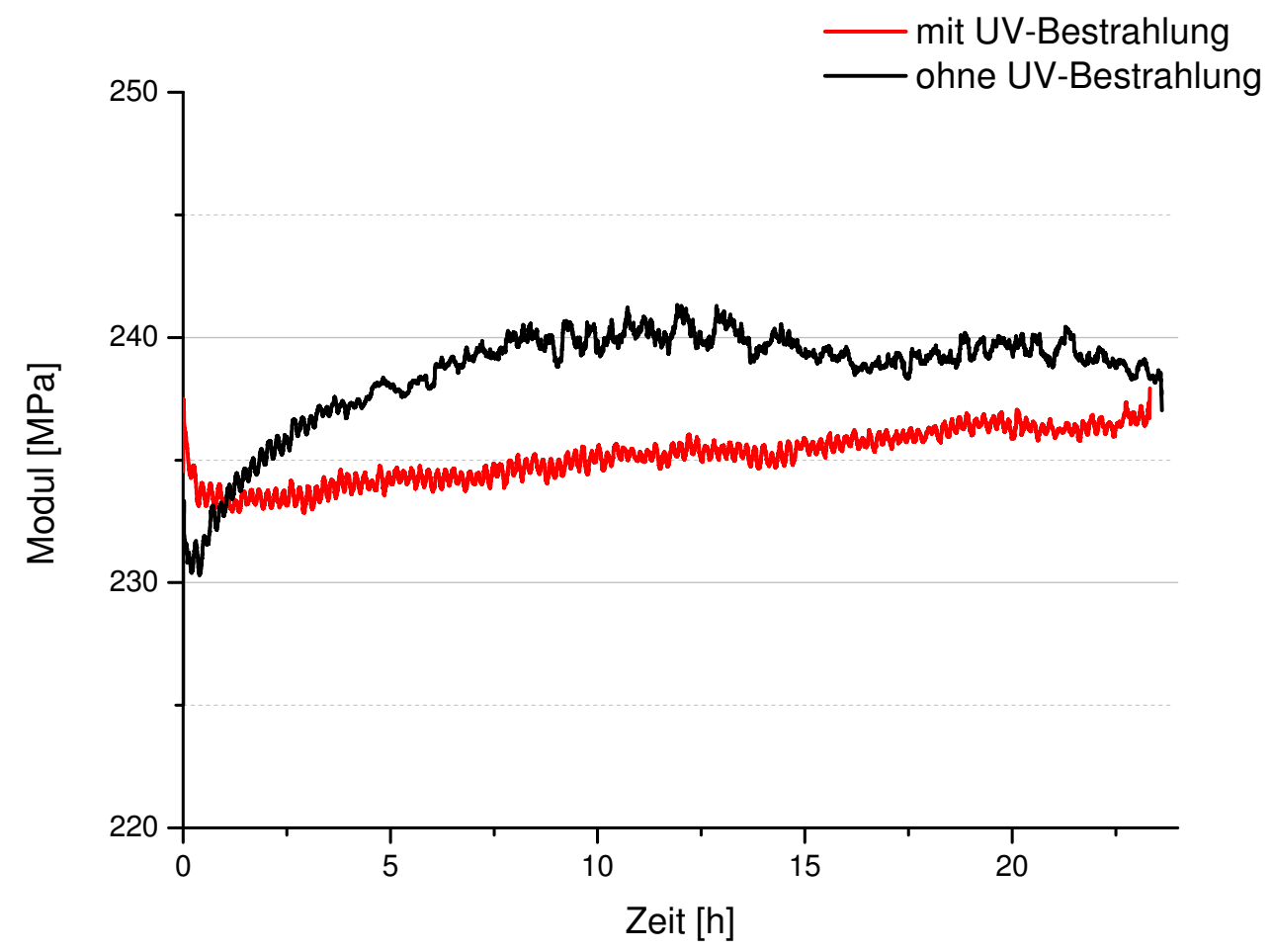

Abb. 5.14.: Speichermodul in Abhängigkeit der Zeit für die Probe 37-08. Es wurde jeweils eine Probe während der Messung dauerhaft mit UV-Licht $\lambda<310 \mathrm{~nm}$ bestrahlt und die andere nicht.

Um den Einfluss der dauerhaften mechanischen Beanspruchung auf das Vernetzungsverhalten auszuschließen wurde daher zudem folgender Intervallversuch durchge- 
führt. Das Material wurde in Zuggeometrie in den DMA8000 eingebaut und der Modul dynamisch bestimmt, indem mit einer Frequenz von $1 \mathrm{~Hz}$ und einer Auslenkung von 0,005 mm gemessen wurde. Dann wurde die Probe jeweils für $1 \mathrm{~h}$ bestrahlt und anschließend wieder der Modul bestimmt. Während der Bestrahlung wurde die Probe nicht dynamisch beansprucht.

In Abbildung 5.15 ist für zwei Proben im Intervallversuch der Speichermodul in Abhängigkeit der Bestrahlungsdauer im abgebildet. Der Modul steigt kontinuierlich mit der Bestrahlungsdauer und erreicht einen deutlichen Zuwachs von 10-15\%. Dies ist ein Hinweis, dass ein Crosslinking tatsächlich stattfindet und zudem einen Einfluss auf die Mechanik hat. Für die Probe mit dem höheren MMA-Anteil ist der Modulzuwachs größer. Dies ist zu erwarten, da die vernetzende Coumarin Einheit nur in den MMA-Block eingebaut ist.

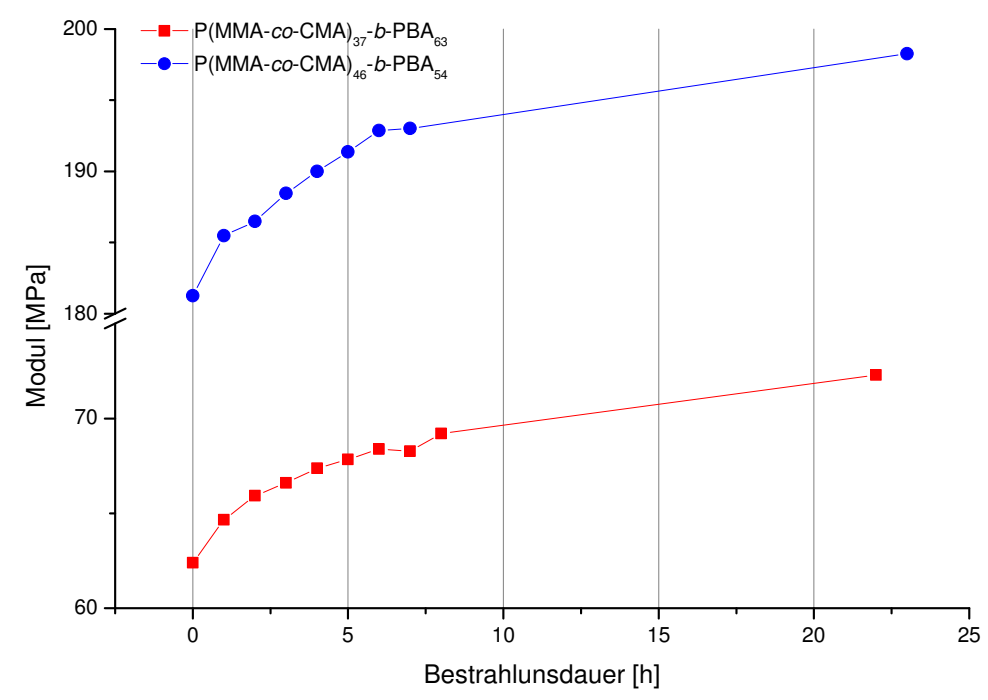

Abb. 5.15.: Modul in Abhängigkeit der Dauer der Bestrahlung mit Licht der Wellenlänge $\lambda>310 \mathrm{~nm}$. Während der Bestrahlung wurden die Proben nicht dynamisch beansprucht.

Die bei Bestrahlung mit $\lambda>310 \mathrm{~nm}$ entstandenen Vernetzungen der CoumarinBindungen, können mit Licht der Wellenlänge $\lambda=254 \mathrm{~nm}$ wieder gelöst werden. Messungen mit dem UV/Vis-Spektrometer, die von K.Tietz durchgeführt worden sind können ein erfolgreiches Öffnen der Crosslinks verifizieren. Jedoch wurde beobachtet, dass für die Reaktion der Dimerspaltung lediglich 13\% der Vernetzungesstellen wieder gelöst werden können [93].

Zur Untersuchung des Einflusses des Decrosslinking Prozesses auf die mechanischen Eigenschaften wurde ein ähnlicher Intervallversuch wie für die Vernetzungsreaktion durchgeführt. Da der erwartete Effekt jedoch sehr gering ist, wurde anstelle eines der Blockcopolymere nur der erste Block P(MMA-co-CMA) untersucht, da hier der stärkste Effekt zu erwarten ist. Die Probe wurde in den DMA eingebaut und war vorher mit Wellenlicht der Wellenlänge $\lambda>310 \mathrm{~nm}$ bestrahlt worden, so dass sie 
zu Beginn des Experimentes möglichst zu einem großen Teil vernetzt ist. Nun wird die Probe für 10 min dynamisch im DMA gemessen und ein Wert für den Modul bestimmt. Anschließend wird die dynamische Anregung abgeschaltet und das Probenmaterial mit Licht der Wellenlänge $\lambda=254 \mathrm{~nm}$ bestrahlt. Dieser Vorgang wurde mehrmals wiederholt. Die resultierenden Ergebnisse sind in der Abbildung 5.16 dargestellt. Es ist eine leichte Abnahme des Moduls mit der Bestrahlungsdauer zu sehen. Dies entspricht den Erwartungen, da beim Lösen der Crosslinks das Material mehr nachgeben kann und es damit zu einer Veringerung des Moduls kommt.

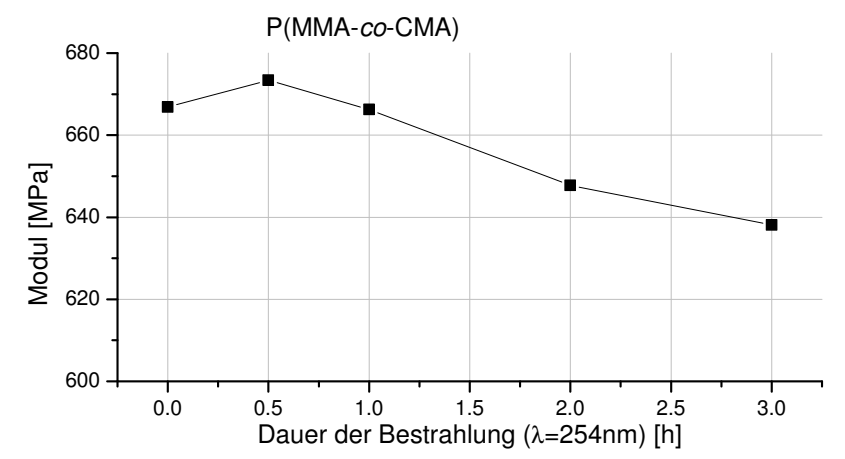

Abb. 5.16.: Abhängigkeit des Moduls von der Dauer der Bestrahlung mit $\lambda=254 n m$

\subsection{Ordnungs-Unordnungs-Übergang}

Wie in Abschnitt 2.3.3 dargelegt wurde, ist das Auftreten eines Ordnungs-Unordnungsüberganges (kurz ODT) charakteristisch für mikrophaseseparierende Blockcopolymere. Mit steigendem $\chi N$ wird das phasenseparierte System in den ungeordneten Zustand überführt. Dies kann entweder über eine Erhöhung der Temperatur $\chi \propto T^{-1}$ oder durch die Reduzierung des Polymerisationsgrades $N$ erreicht werden. Als Vorraussetzung um einen ODT zu detektieren, muss dieser jedoch in einem Temperaturbereich stattfinden, der unterhalb der Degradationstemperatur des Polymersystems liegt. Da in der im vorigen Abschnitt untersuchten Probencharge bisher keine eindeutigen Hinweise auf einen ODT aufgetreten sind, muss angenommen werden, dass der im Experiment zugängliche Temperaturbereich noch unterhalb des Bereiches liegt, in dem die Polymere entmischen würden. Daher wurde ein Blockcopolymer X mit deutlich geringerem Polymerisationsgrad hergestellt.

Zunächst wurde die Signatur eines ODT mit dem DMA untersucht, indem ein kommerziell hergestelltes Blockcopolymer Polystyrene-Polyisoprene von Polymersource ${ }^{\mathrm{TM}}$ gemessen wurde. Dieses Blockcopolymersystem ist in der Literatur dafür bekannt einen ODT zu zeigen [57]. Das Material lag in Pulverform vor und wurde analog zu den im Abschnitt 5.2 gezeigten Ergebnissen in einem Material Pocket in einem Biegebalkenversuch mit einer dynamischen Auslenkungsamplitude von 0,02 mm und einer Frequenz von $5 \mathrm{~Hz}$ untersucht. Dabei wurde die Temperatur mit einer Rate von 


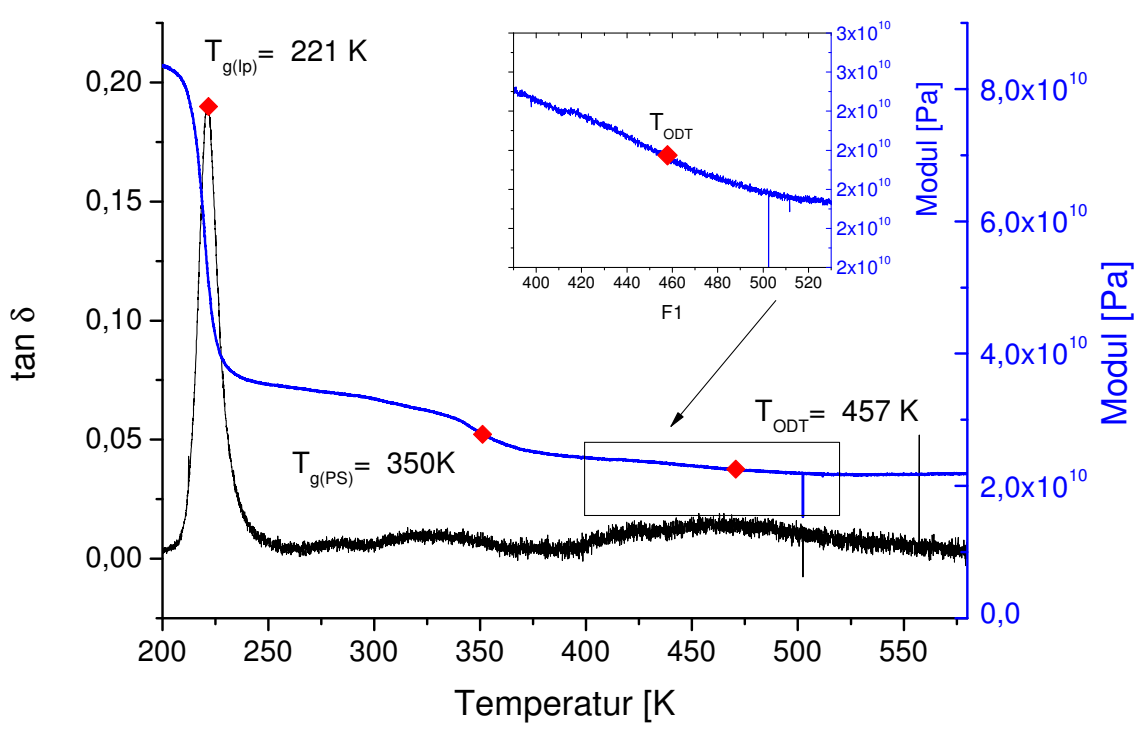

Abb. 5.17.: Temperaturscan des PSPI-Blockcopolymers. Es sind der $\tan \delta$ und Modul aufgetragen. Das Inset zeigt einen vergrößerten Auschnitt für den ODT.

$2 \mathrm{~K} / \mathrm{min}$ erhöht. Der temperaturabhängige tan $\delta$ und Modul aus dieser Messung sind in der Abbildung 5.17 dargestellt.

Der Temperaturscan zeigt eindeutig den Glasübergang von Polystyrene bei $350 \mathrm{~K}$ (Literaturwert: 357K), sowie den Glasübergang von Polyisoprene bei 221K (Literaturwert : 212K). Zusätzlich ist ein relativ breiter Übergang bei Temperaturen oberhalb der beiden Glasübergangstemperaturen der einzelnen Blöcke zu sehen. Dieser Übergang stellt den ODT dar und liegt für dieses Probensystem bei einer Temperatur von $457 \mathrm{~K}$. Dies ist in guter Übereinstimmung mit anderen Messungen an diesem Probensystem [57].

Es wurde für das hier untersuchte $\mathrm{BCP} \mathrm{P}(\mathrm{MMA}-c-\mathrm{CMA})-b$-BA eine Probe mit einem geringeren Polymerisationsgrad hergestellt um den ODT experimentell zugänglich zu machen. Die Probe X weist eine kürzer Kettenlänge auf und besitzt einen BA-Anteil von $69 \%$. Sie wurde auf einen ähnlich gearteten Übergang wie das PSPI-System untersucht und es konnte hier erfolgreich ein ODT detektiert werden. Abbildung $\underline{5.18}$ zeigt einen Temperaturscan für den Temperaturbereich zwischen Raumtemperatur und $553 \mathrm{~K}$. Es kann neben dem Glasübergang des MMA-Blockes zusätzlich ein weiterer relativ breiter Übergang detektiert serden, der mit dem ODT identifiziert werden kann.

Die Detektion eines ODT für dieses Probensystem konnte zudem zusätzlich mit AFM-Messungen aus [93] bestätigt werden. Dafür wurde das folgende Verfahren benutzt. Es wurden zunächst zwei identische Proben Xa und Xb bei Raumtemperatur mit dem AFM untersucht, wobei eine mikrophasenseparierte Struktur beobachtet 


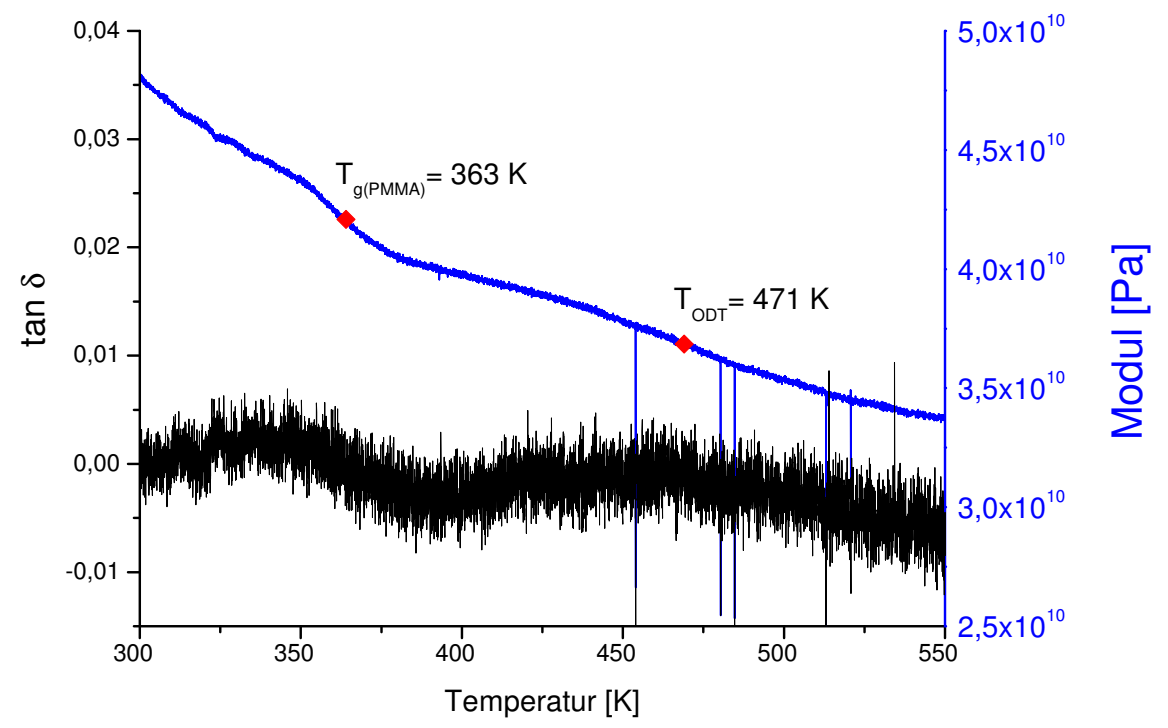

Abb. 5.18.: DMA-Temperaturscan der Probe X. Es sind der temperaturabhängige tan $\delta$ und der Modul dargestellt.

wurde (Abbildung 5.19 a1) und b1). Anschließend wurden die Proben auf $453 \mathrm{~K}$ geheizt und $4 \mathrm{~h}$ bei dieser Temperatur gehalten. Die Probe Xa wurde während dieses Temperns zusätzlich mit Licht der Wellenlänge $\lambda>310 \mathrm{~nm}$ bestrahlt, so dass das Material vernetzt und damit die Morphologie „eingefroren“ wird. Anschließend wurden beide getemperten Proben wieder mit dem AFM bei Raumtemperatur untersucht. Die mit dem AFM bestimmten Topographien für beide Proben vor und nach dem Tempern sind in der Abbildung 5.19 dargestellt. Nach dem Tempern zeigt die vernetzte Probe Xa2 einen ungeordneten Zustand, während die unvernetzte Probe $\mathrm{Xb} 2$ wieder in einem mikrophasenseparierten Zustand vorliegt. Dies bedeutet, dass die Vernetzung erfolgreich war und dass ein ODT bei einer Temperatur $T<453 \mathrm{~K}$ stattfindet. 

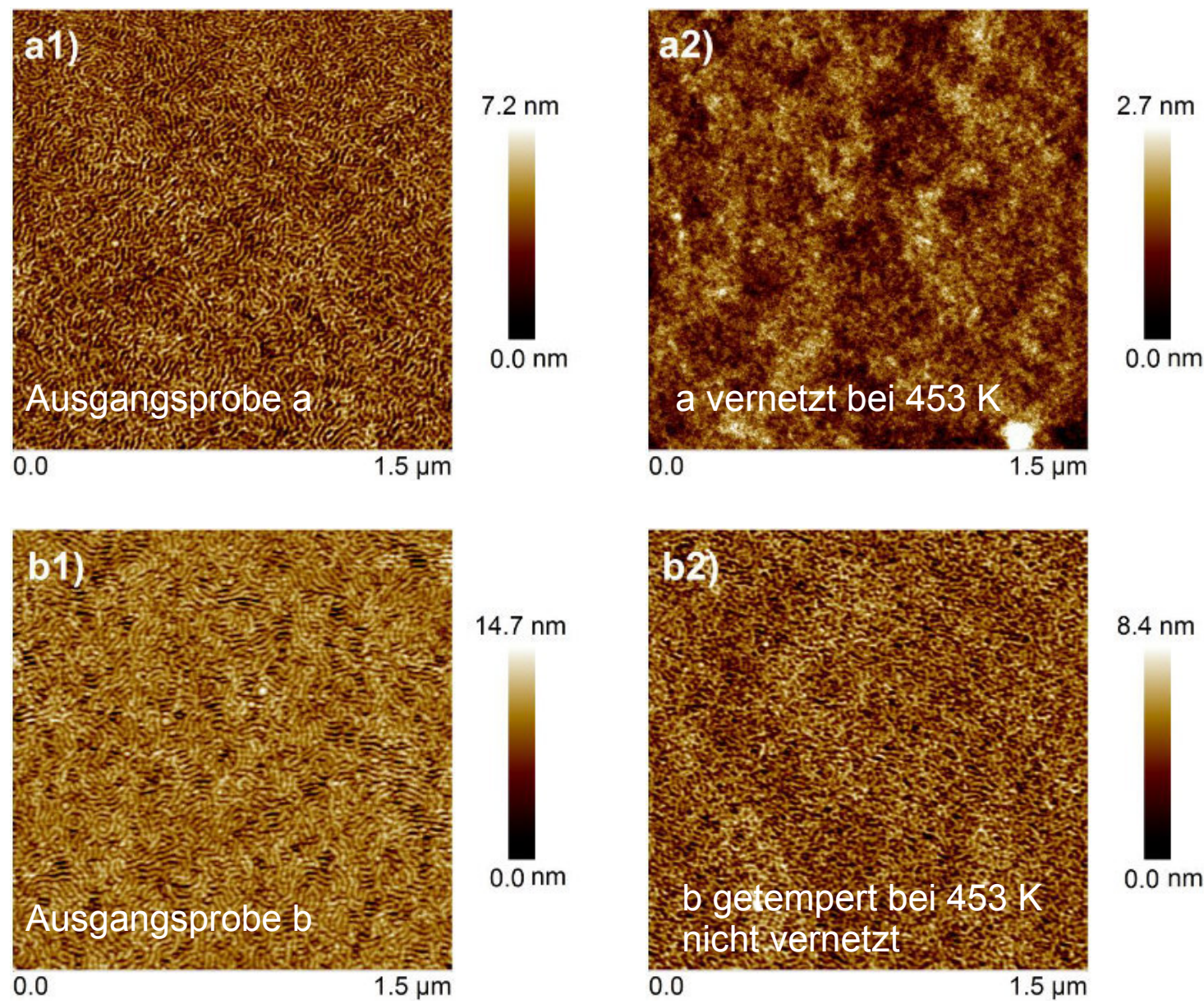

Abb. 5.19.: AFM-Höhenprofile des BCPS X (70 mol \% BA) gemessen bei $294 \mathrm{~K}$ : a1) Ausgangsprobe Xa b1) Ausgangaprobe Xb a2) Probe Xa nach 4 h Bestrahlung mit $(\lambda<$ $310 \mathrm{~nm}$ ) bei $453 \mathrm{~K}$ b2) Probe Xb nach $4 \mathrm{~h}$ Tempern bei $453 \mathrm{~K}$ aus [93] 


\subsection{Zusammenfassung der Ergebnisse}

Es wurden Blockcopolymere P(MMA-c-CMA)- $b$-BA auf ihre thermischen Eigenschaften untersucht. Zunächst konnte anhand von TGA-Messungen gezeigt werden, dass die Degradation der Polymere erst bei Temperaturen oberhalb, des für die DMA-Messungen verwendeten Temperaturbereiches, einsetzt. Die Homopolymere des Blockcopolymers zeigten im DMA-Temperaturscan jeweils einen Glasübergang in den aus der Literatur bekannten Bereichen. Für den P(MMA-co-CMA)-Block wurde zudem der Einfluss des Molgewichtes, sowie des Coumerins bestimmt. Dabei steigt die Glastemperatur mit dem Molgewicht und das Coumarin führt zu einer Senkung der Glastemperatur. Desweiteren wurde die temperaturabhängigen mechanischen Eigenschaften des Blockcopolymers für fünf verschiedene Zusammensetzungen untersucht. Dabei konnten jeweils die beiden Glastemperaturen der einzelnen Blöcke beobachtet werden. SAXS-Messungen zeigen, dass für viele der Probe eine Nahordnung im Bulk besteht. Für Proben mit einem sehr hohen BA-Gehalt konnte zusätzlich noch ein schwacher Peak bei kleineren q-Werten gemessen werden, was auf eine Fernordnung hinweist. Das erfolgreiche Vernetzen der Proben mit UV-Licht konnte mit dem DMA beobachtet werden. Einerseits zeigt der Vergleich von DMAMessungen einer vernetzten und einer unvernetzten Probe die Unterdrückung der Relaxation. Zudem wurde gezeigt, dass die Vernetzung unter dynamischer Beanscpruchung deutlich weniger effektiv ist, als die Vernetzung im Ruhezustand. Denn für die Probe unter dauerhafter dynamischer Belastung konnte ein Modulzuwachs von etwa $1 \%$ festgestellt werden, während es im Intervallversuch bis zu $25 \%$ waren. Das Lösen der Vernetzungstellen konnte am P(MMA-co-CMA)-Block erfolgreich gezeigt werden. Der Effekt ist deutlich geringer, als beim Vernetzen und führt zu einer Abnahme, des Moduls. Der Ordnungs-Unordnungsübergang wurde mit dem DMA an einem kommerziellen Blockcopolymer PSPI untersucht und auch erfolgreich bei einem Blockcopolymer P(MMA- $c$-CMA)- $b$-BA mit geringem Molgewicht beobachtet.

\subsection{Diskussion}

In diesem Abschnitt sollen die im Kapitel $\underline{5}$ dargestellten Ergebnisse diskutiert werden. Zunächst soll ein Phasendiagramm der beobachteten Morphologien erstellt werden, indem die mit dem DMA gemessenen Temperaturscans mit den AFM-Bildern aus [93] verglichen werden. Zusätzlich werden in diesem Zusammenhang die Ergebnisse der Kleinwinkelröntgenstreuung diskutiert. Anschließend folgt ein Vergleich der globalen und der lokalen mechanischen Eigenschaften der Proben. Folgend wird dass Vernetzen, sowie Entnetzen der P(MMA-c-CMA)-Blöcke mittels Bestrahlung von UV-Licht behandelt. Im letzten Abschnitt wird schließlich das Auftreten eines ODT diskutiert. 


\subsubsection{Morphologie}

Es wurde ein Blockcopolymersystem mithilfe von temperaturabhängigen dynamisch mechanischen Messungen hinsichtlich Mikrophasenseparation untersucht. Zunächst wurden die einzelnen Komponenten des Systems untersucht und die Glasübergangstemperaturen der Homopolymere $\mathrm{P}(\mathrm{MMA})$ und $\mathrm{P}(\mathrm{BA})$ bestimmt. Diese sind in guter Übereinstimmung mit Literaturwerten. Die Abweichungen sind darauf zurückzuführen, dass der Glasübergang einerseits stark von der Polymerarchitektur abhängt, welche entscheidend durch den Herstellungsprozess beeinflusst wird. Zudem ist der Glasübergang generell abhängig von der gewählten Messmethode und den dabei vewendeten Parametern.

Da das Blockcopolymer mithilfe von UV-Licht vernetzt werden soll, wurden photovernetzbare Coumarin-Einheiten statistisch in den $\mathrm{P}(\mathrm{MMA})$-Block eingebaut. Der Einfluss des CMA auf die Temperaturabhängigkeit der Mechanik wurde untersucht und es wurde beobachtet, dass das Coumarin zu einer Verschiebung der Glastemperatur des MMA-Blockes zu niedrigeren Temperaturen führt. Weiterhin konnten an den P(MMA-co-CMA) Polymeren der Einfluss der Kettenlänge auf den Erweichungspunkt beobachtet werden. Die kurzkettigen Polymere zeigen wie erwartet einen Glasübergang bei kleineren Temperaturen.

Die Blockcopolymere P(MMA-co-CMA)- $b$-PBA wurden ebenfalls mit dem DMA auf die Temperaturabhängigkeit der mechanischen Eigenschaften untersucht. Bei diesen Messungen konnten jeweils zwei Glasübergangstemperaturen bestimmt werden, die jeweils mit den Glastemperaturen der einzelnen Blöcke identifiziert werden konnten. Dies ist ein Hinweis auf ein mikrophasenseperiertes System. Im Vergleich dazu ist in der Literatur bekannt, dass statistische Copolymere aus BA und MMA lediglich eine einzelne Glastemperatur zeigen, welche sich zwischen den beiden Glastemperaturen der Homopolymere befindet. Mit steigendem BA-Gehalt verschiebt sich diese Mischungsglastemperatur immer weiter $\mathrm{zu} \operatorname{Tg}(\mathrm{BA})$ [29].

Die mit dem DMA untersuchten Blockcopolymerproben sind in der Arbeit [93] von K.Tietz mit einem Rasterkraftmikroskop untersucht worden. Im Folgenden sollen diese AFM-Messungen mit den im Ergebnisteil dargestellten Temperaturscans am DMA verglichen werden und die Eigenschaften der verschiedenen Morphologien diskutiert werden und im Anschluss ein Phasendiagramm erstellt werden.

D (37-08) 54\% BA Die Probe D liegt mit einem BA-Anteil von 54\% in nahezu symmetrischer Zusammensetzung vor. Bei dieser Probe konnten im Temperaturscan die beiden Glastemperature der Homoploymere BA und MMA beobachtet werden. Dabei zeigte jedoch der relativ breite MMA-Übergang bei genauerer Analyse drei schwach ausgeprägte Stufen im Speichermodul. Diese Stufen könnten auf Relaxationen innerhalb der Struktur hindeuten. Da auch in dem geometrieunabhängigen Verlustwinkel $\tan \delta$ eine Signatur zu sehen ist, muss angenommen werden, dass es sich hier nicht um Artefakte handelt. Die Abbildung $\underline{5.20}$ zeigt einen vergrößer- 


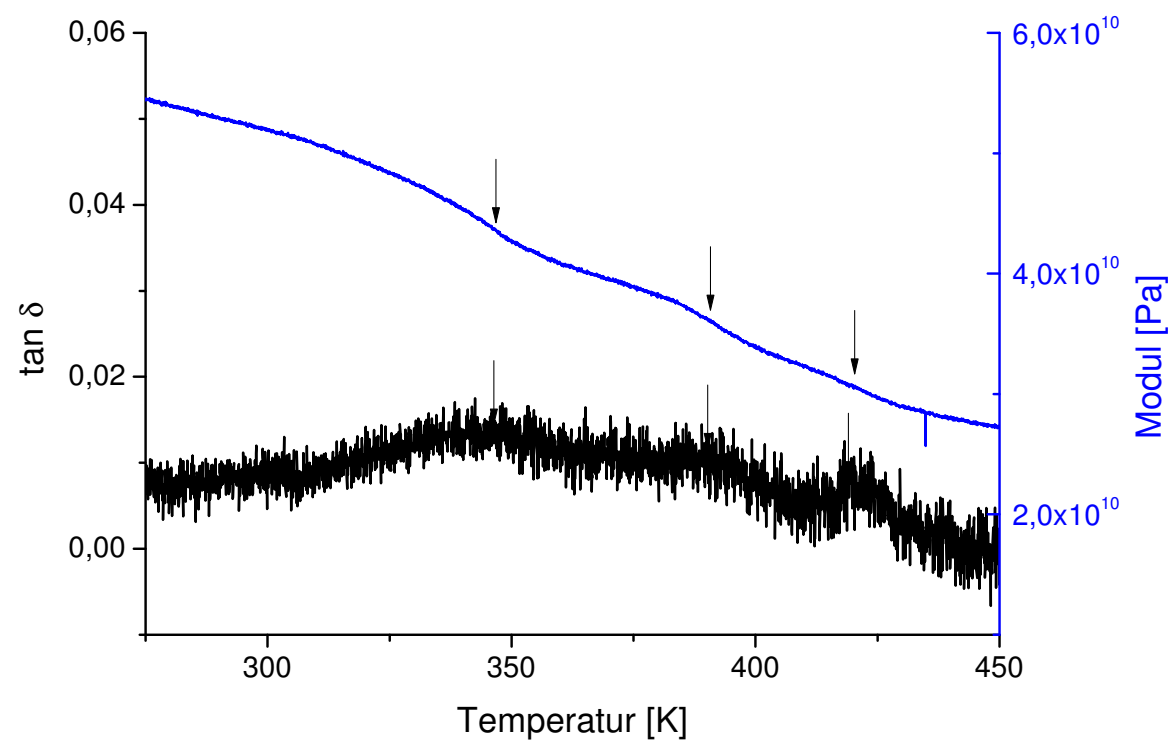

Abb. 5.20.: Vergrößerter Ausschnitt aus dem DMA-Temperaturscan der Probe D(37-08).

ten Ausschnitt aus dem Temperaturscan. In der Abbildung sind im $\tan \delta$ und im Speichermodul jeweils die Prozesse mit einem Pfeil gekennzeichnet. Bei einem der Übergänge muss es sich um das Erweichen der MMA-Blöcke handeln. Dabei kann dies entweder der Übergang bei $347 \mathrm{~K}$ oder der bei $391 \mathrm{~K}$ sein. Im Vergleich mit Probe E, welche eine ähnlich Zusammensetzung aufweist, wurde der Glasübergang des MMA-Anteiles bei einer Temperatur von 346K gefunden. Daraus wird gefolgert, dass der Übergang bei 347K, den Glasübergang des PMMA-Blockes darstellt. Dies ist plausibel, da erst durch die Mobilität, der MMA-Blöcke weitere Umstrukturierungen im Material ermöglicht werden, die sich in der Messung dann bei den beiden höheren Temperaturen zeigen. Die Natur der beiden Prozesse bei 391K und 424K soll anhand der AFM-Bilder von K.Tietz für diese Probe diskutiert werden. Die Abbildung5.21 zeigt AFM-Aufnahmen der Topologie der Proben einmal vor dem Tempern und einmal nach dem Tempern bei 433K. Während des Temperns konnte das Polymer relaxieren und seine Gleichgewichtsmorphologie einnehmen. Diese Morpholgie ist die von perforierten Lamellen, die horizontal zum Substrat verlaufen. Damit wird für diese Probe eine strukturelle Relaxation im Temperaturbereich unterhalb 433K erwartet, die zu der Gleichgewichtsmorphologie führt. Es wird jedoch im DMA ein weiterer Prozess beobachtet. Für diesen können zwei verschiedene Szenarien verantwortlich sein. Zunächst könnte es sich um den ODT handeln. Um dies zu beobachten, müsste die Struktur oberhalb von $433 \mathrm{~K}$ untersucht werden. Es liegen jedoch temperaturabhängige AFM Untersuchungen von K.Tietz an der Probe F(30-05) vor, die selbst bei Temperaturen von bis zu 473K keinen ungeordnete Struktur zeigten. Im Vergleich zur hier dskutierten Probe D hat diese Probe $\mathrm{F}$ jedoch ein um 58\% geringere Molmasse, also einen deutlich geringeren Polymeri- 

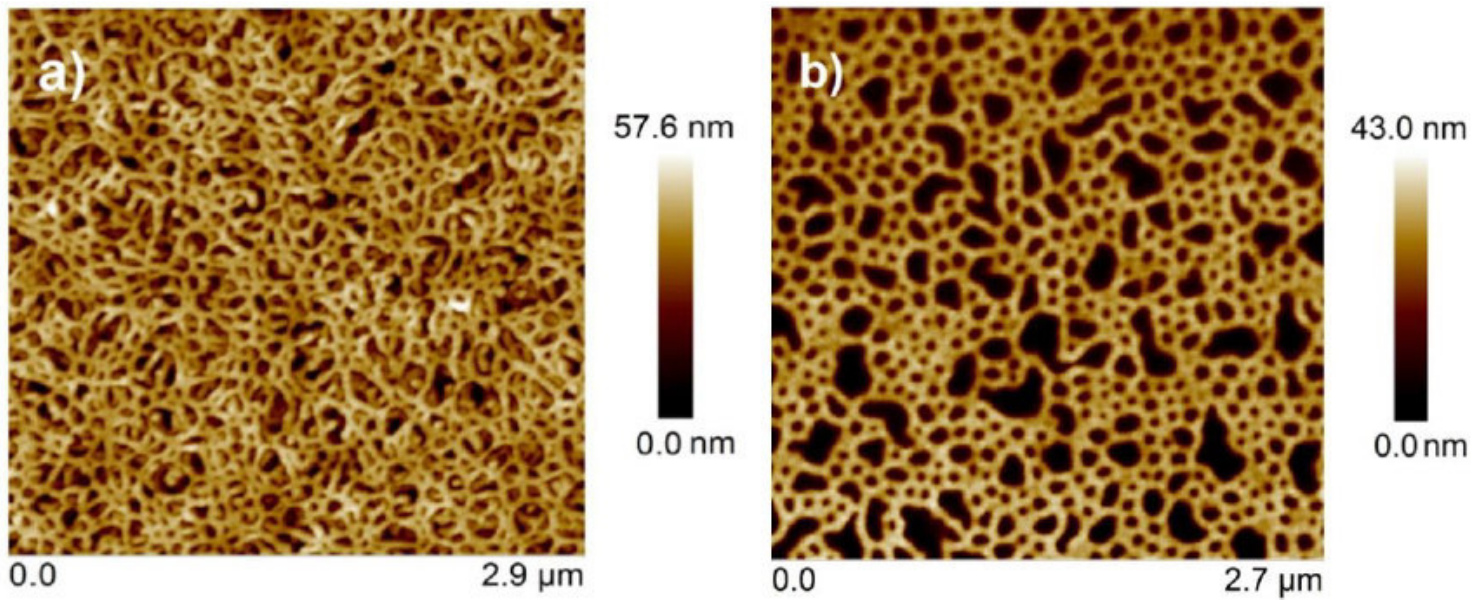

Abb. 5.21.: AFM-Aufnahme der Topographie der Probe G(37-8) a) vor dem Tempern bei $433 \mathrm{~K}$ b) nach dem Tempern.

sationsgrad N und sollte damit im Phasendiagramm näher am ODT liegen. Damit ist es unwahrscheinlich, dass der im DMA beobachtete Übergang der ODT ist. Ein andere Ursache könnte der Übergang im Phasendiagramm von einer Morphologie zu einer anderen sein. In der Veröffenlichung [86] konnten nämlich für diese Zusammensetzung neben der Struktur einer perforierten Lamelle zudem ein Zylinderstruktur beobachtet werden. Die SAXS-Messungen zeigten lediglich einen Peak bei einer Länge von $14 \mathrm{~nm}$. im AFM wurde jedoch eine charakteristische Längenskala von 86nm beobachtet. Die mit den SAXS untersuchte Probe liegt entweder noch nicht in der Gleichgewichtsmorphologie vor, so dass erst ein längerer Temperprozess zu einer Fernordnung führen würde oder die beobachtete Struktur ist nicht perfekt ausgebildet, da sich die Probe direkt auf einer Phasengrenze zwischen der Struktur einer perforierten Lamelle und der Zylinderstruktur befindet.

E 37-10 Diese Probe zeigt im DMA ebenfalls keine einzelne Stufe für den PMMAGlasübergang, sondern zwei einzelne Prozesse. Ähnlich wie bei Probe E, kann hier einer der Übergänge als das Erweichen der PMMA-Blöcke interpretiert werden, während der andere eine struktrurelle Relaxation darstellt, welche zur mikrophasenseparierten Gleichgewichtsmorphologie führt. Im AFM konnte für diese Probe ebenfalls die Struktur von perforierten Lamellen gefunden werden. Die SAXS-Messung jedoch zeigte keine Peaks, was auf einen unzureichenden Temperprozess zurückzuführen sein könnte.

F 30-05 für diese Probe konnten ebenfalls zwei Glasübergänge bestimmt werden. Jedoch war der PMMA-Übergang sehr breit. Dies kann an der zugrundeliegende Gleichgewichtsmorpholgie liegen. K.Tietz konnte für diese Probe die Struktur von liegenden PMMA-Zylindern in einer BA-Matrix finden. „Schwimmen „, diese Zylinder 

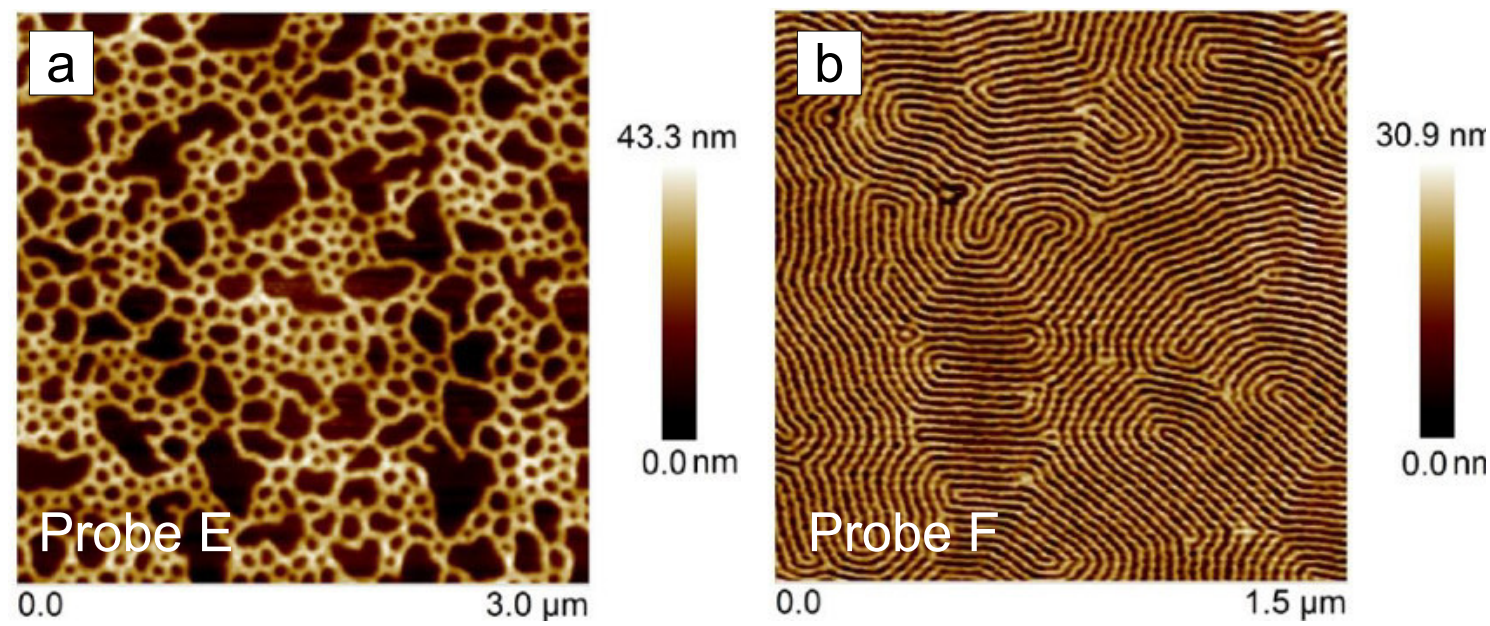

Abb. 5.22.: Topographien der Proben a) E (37-10) (61\%BA) und b) F(30-05)(68\%BA)

in der Ba-Batrix und bilden kein verbundenes Netzwerk, so kann es sein, dass das Erweichen der Zylinder nur eine geringe Änderung der mechanischen Eigenschaften bewirkt.

G 47-01 und H 47-03 Für diese Proben wird auch ein schwach ausgeprägter MMA-Übergang gefunden, welcher durch die Morphologie der Proben hervorgerufen sein kann. Diese Proben zeigen im AFM-Bild Sphären von dem harten PMMA, die in der weichen Matrix des BA sehr regelmäßig auf einem hexagonalen Gitter geordnet sind. Dadurch haben diese harten Kugeln keinerlei Kontakt untereinander und beeinflussen die mechanischen Eigenschaften der Probe kaum. Dadurch hat das Weichwerden dieser Kugeln nur einen geringen Effekt auf die mechanischen Eigenschaften.

Die Abbildung 5.23 zeigt eine Übersicht der gefundenen Morphologien für verschiedene Zusammensetzungen. Da in der Veröffentlichung von [86] ebenfallls ein Blockcopolymer PMMA- $b$-PBA untersucht wurde, sind die von Siprom et al. gefundenen Morphologien auch eingetragen.

Für Proben mit einem geringen BA-Anteil liegen in dieser Arbeit keine Untersuchungen vor, da sich im Experiment gezeigt hat, dass diese PMMA-reichen Proben aufgrund ihrer Glastemperatur oberhalb von Raumtemperatur sehr eingeschränkt sind und somit die Gleichgewichtsmorphologie schwerer zugänglich ist. Siprom et al. fanden jedoch für Zusammensetzungen mit einem BA-Anteil kleiner als $50 \%$ eine Struktur von parallelen Lamellen.

Fazit Es wurden Blockcopolymere verschiedener Zusammensetzung auf ihre temperaturabhängigen mechanischen Eigenschaften untersucht. Dabei konnten verschiedene Prozesse gefunden werden. Einerseits konnten die Glasübergänge der einzelenen Homopolymere beobachtet werden, was das Vorliegen einer mikrophasenseparierten 
5.7. Diskussion

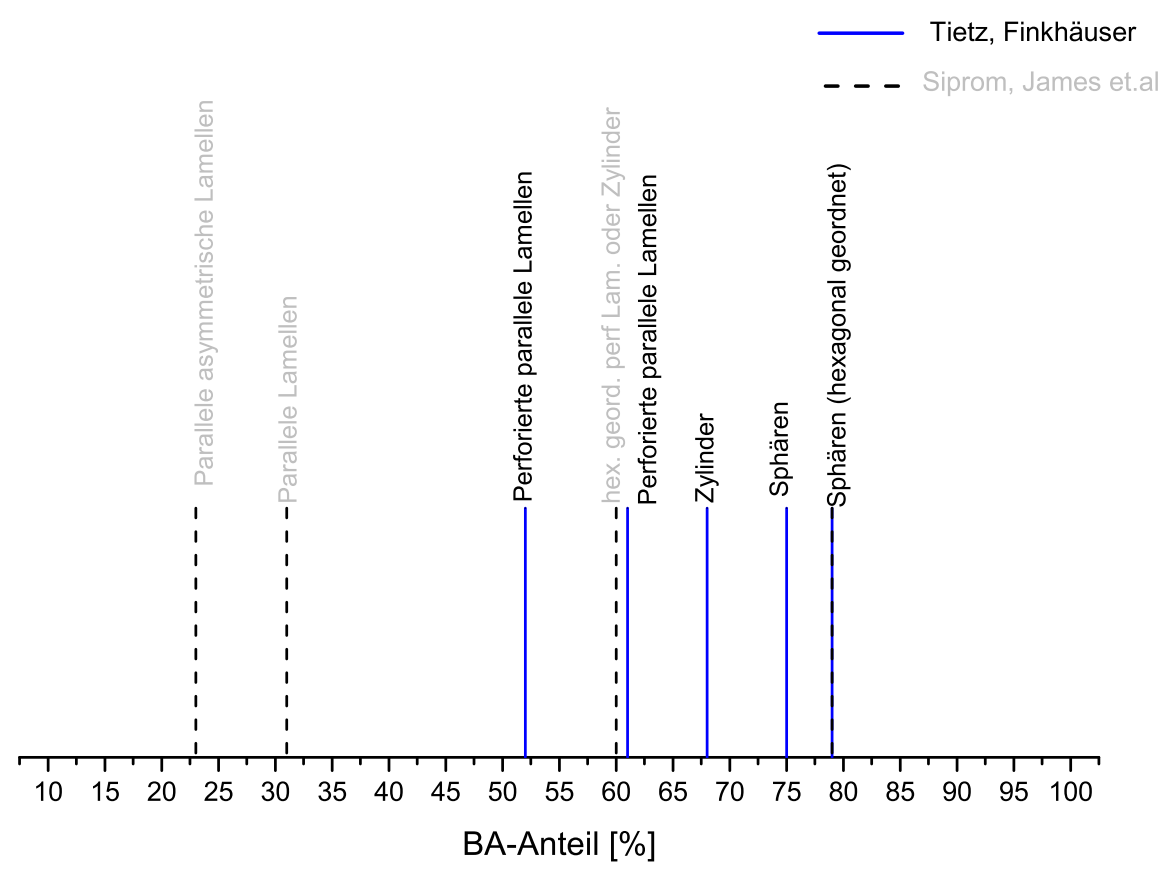

Abb. 5.23.: Morphologien für verschiedene Zusammensetzungen des BCPs P(MMAco-CMA)-b-BA. Die gestrichelt gekennzeichneten Morphologien sind von Sriprom et. al aus[86] entnommen. 


\section{UV-aktive Blockcopolymere}

Morphologie bestätigt. Andererseits wurden zusätzliche Prozesse beobachtet, die anhand des Vergleiches mit den AFM Messungen als Relaxationsprozesse zur Gleichgewichtsmorphologie identifiziert werden konnten. Kleinwinkelstreuung zeigte zudem, dass eine Nahordnung in den Proben zu beobachten ist und für einige konnte auch eine Fernordnung beobachtet werden.

\subsubsection{Vernetzung und Entnetzung mit UV-Licht}

Es wurden Blockcopolymere $\mathrm{P}(\mathrm{MMA}-c o-\mathrm{CMA})-b$-PBA zunächst hinsichtlich der UV-induzierten photochemischen Vernetzung untersucht. Die Vernetzung wurde dabei jeweils im festen Zustand und nicht in Lösung durchgeführt. Dass die Vernetzungsreaktion stattfindet und dabei eine Effektivität von $40 \%$ bei Bestrahlung bei Raumtemperatur aufweist wurde anhand UV/Vis-Spektrometrie von K.Tietz für dünne Polymerfilme gezeigt. Der Vernetzungsprozess zeigte dabei, bei einer Vernetzungstemperatur von $398 \mathrm{~K}\left(>T_{g}(\mathrm{PMMA}), T_{g}(\mathrm{BA})\right)$ eine höhere Effektivität von $66 \%$, was auf die erhöhte Mobiltät der Polymere zurückzuführen ist.

Es wurden in dieser Arbeit zunächst Proben untersucht, die vor dem Einbau in den DMA mit UV-Licht $(\lambda<310 \mathrm{~nm})$ bei Raumtemperatur bestrahlt wurden. Es wurde jeweils für das bestrahlte und das unbestrahlte Probenmaterial ein Temperaturscan aufgenommen. Für die unbestrahlten Proben konnte festgestellt werden, dass sich beim ersten Heizen der Peak im tan $\delta$, der Erweichungstemperatur des PMMAs weniger stark ausgeprägt war, als bei dem anschließenden Kühlvorgang. Dies ist ein Hinweis, dass durch den Heizprozess das Material relaxieren konnte. Da es sich um ein mikrophasenseparierendes Blockcopolymersystem handelt, ist anzunehmen, dass diese Relaxation mit der Bildung der Gleichgewichtsmorphologie der Probe zusammenhängt. Durch das Heizen bis oberhalb der Glastemperaturen von beiden Blöcken, ist das Polymer so mobil, dass sich die PMMA-Blöcke soweit von dem BA entmischen, wie es trotz ihrer kovalenten Bindung möglich ist. Dies führt dazu, dass nach dem Heizen aufgrund der besseren Entmischung der BA und der PMMA-Phase, das Erweichen der PMMA-Blöcke eindeutiger im DMA-Temperaturscan zu beobachten ist. Die bestrahlte Probe hingegen kann nicht relaxieren, da die Bewegung der PMMA-Blöcke aufgrund der Vernetzungen beschränkt ist. Das Heizen weit über die Glastemperatur der beiden Komponenten führt offensichtlich nicht zu einer Relaxation und damit wird die Bildung der Gleichgewichtsmorphologie unterdrückt. Dieser Effekt konnte von K.Tietz für die Oberfläche mit dem AFM beobachtet werden. Dafür wurden vernetzte und unvernetzte Proben bei 433K getempert und anschließend mit dem AFM untersucht. Dabei wurde für die unvernetzte Probe eine signifkant andere Topologie gefunden, als für das vernetzte Material, welches die gleiche Topologie wie vor dem Temperprozess zeigte [93]. Dies bietet viele interessante Möglichkeiten, da es hierdurch möglich ist, direkt Zustände bei verschiedenen Temperaturen festzuhalten, indem das Material bei einer Temperatur bestrahlt wird und es bei einer anderen Temperatur untersucht wird. Vor allem in Hinblick auf Untersuchungen mit 
dem AFM, welches häufig bei Raumtemperatur betrieben wird, sind auf diese Weise nun auch die Topologien, bei höherer Temperatur zu untersuchen. Dieses Verfahren wird wie in Abschnitt 5.5 beschrieben, im Zusammenhang mit der Detektion des ODT verwendet.

Der Einfluss der UV-induzierten photochemischen Vernetzung auf die globalen mechanischen Eigenschaften konnte an zwei Blockcopolymerern P(MMA-co-CMA)- $b$ PBA D(52\%BA) und E(61\%BA) in einem Zugversuch mit dem DMA untersucht werden. Es wurde zunächst ein Dauerversuch durchgeführt, bei dem die Probe dauerhaft dynamisch mechanisch mit der Frequenz $1 \mathrm{~Hz}$ beansprucht wurde und simultan mit UV-Licht der Wellenlänge $\lambda>310 \mathrm{~nm}$ bestrahlt wurde. Dabei konnte im Gegensatz zu den im folgenden diskutierten Intervallversuchen, bei denen die Bestrahlung im Ruhezustand stattfand, kein signifikanter Anstieg im Modul beobachtet werden. Dies könnte einerseits auf den mit der Verfestigung aufgrund der Bestrahlung konkurrierenden Effekt einer mechanischen Verformung des Materials zurückzuführen sein. Die untersuchten Proben enthalten zwischen $50 \%$ und $62 \%$ Butylacrylat, welches bei Raumtemperatur in seiner flüssigen Phase vorliegt. Damit sind die Proben sehr viskos und können bei Dauerbeanspruchung zu irreversiblen plastischen Verformungen führen. Da jedoch die Vergleichsmessung mit dauerhafter dynamischer Beanspruchung und ohne UV-Bestrahlung kein Absinken des Speichermoduls zeigt, ist dieser Effekt zu vernachlässigen.

Ein andere Ursache für den konstanten Modul im Dauerversuch kann mikroskopischer Natur sein. Die dauerhafte dynamische Belastung im Versuch beeinflusst das Diffusionsverhalten der Polymere und kann damit die Vernetzung behindern. Der Prozess der photochemischen Dimerisierung verläuft nämlich diffusionskontrolliert [3]. Das bedeutet die Diffusionsgeschwindigkeit der Coumarin-Segmente bestimmt die Geschwindigkeit der Dimerisierungsreaktion. Sobald sich zwei Coumarine aufgrund der Diffusionsbewegung der Polymerkette in unmittlebarer Nähe befinden, können sie miteinader reagieren, dabei verläuft die eigentliche Reaktion auf einer Zeitskala von Pikosekunden [63]. Schmidt et al. [83] konnten an Versuchen mit Coumarinsäure im Festkörper zeigen, dass der Abstand der Coumarindoppelbindungen für den Prozess der Photodimerisierung $<4,2 \AA$ betragen muss und dass die Doppelbindungen zudem parallel zueinander ausgerichtet sein müssen um zu dimerisieren.

Es wurde ein Intervallversuch durchgeführt, bei dem das Material für 10min dynamisch beansprucht wurde und daraus der Speichermodul bestimmt. Anschließend wurde das Material der UV-Strahlung $(\lambda<310 \mathrm{~nm})$ ausgesetzt und dann jeweils wieder der Modul bestimmt. Dabei konnte ein Anstieg des Speichermoduls mit steigender Bestrahlungsdauer festegestellt werden. Dies zeigt deutlich, dass Vernetzungsprozesse im Material stattfinden, so dass das Material eine größere Steifigkeit erhält. In der Literatur konnte dieser Effekt bisher auch schon an Nanokompositen beobachtet werden [11]. Da die Vernetzer im harten PMMA-Block integriert sind bildet sich durch die Vernetzung dieser Blöcke ein rigides PMMA-Netzwerk, in der weichen Butylacrylat-Matrix. Die Probe D zeigt einen höheren Modulzuwachs durch 


\section{UV-aktive Blockcopolymere}

die Bestrahlung, als die Probe E. Dies ist auf den höheren MMA-Anteil in der Probe D zurückzuführen, da die Vernetzer nur in den MMA-Block eingebaut sind. Somit kann für diese Probe auch ein höhere Anteil von Venetzungen erreicht werden. Die Effektivität der Dimerbildung verläuft diffusionskontrolliert und hängt damit von der Mobilität der Coumarineinheiten in der Polymerkette ab. Die folgenden Faktoren haben Einfluss auf die Mobilität der eingebauten Coumarine. Zunächst findet die Bestrahlung in der Schmelze und nicht in Lösung statt, wobei die höchste Beweglichkeit der Polymerketten in Lösung ist. Desweiteren befindet sich der MMA-Block während der Bestrahlung ca. 80K unterhalb seiner Glastemperatur. Huyck et al. konnten jedoch zeigen, dass die Photodimerisierungsrate viel geringer ist, wenn sich das Polymer während der Bestrahlung unterhalb seiner Glastemperatur befindet [47].

Die durch die Bestrahlung entstandenen Crossslinks sollen mit mit Licht der Wellenlänge $\lambda=254 \mathrm{~nm}$ wieder gelöst werden können, da hierdurch die entstandenen Dimer zwischen den Coumarineinheiten wieder gespalten werden können. UV/VisMessungen von K.Tietz konnten diesen Effekt zeigen, jedoch mit einer geringen Effektivität von lediglich 13\%. Messungen mit dem DMA konnten eine Abnahme des Moduls zeigen. Der Effekt ist jedoch sehr gering, im Vergleich zu der eindeutigen Veränderung des Speichermoduls während der Vernetzungsreaktion. Die geringere Effektivität im Gegensatz zur Vernetzungsreaktion kann darauf zurückgeführt werden, dass bei Bestrahlung mit Licht der Wellenlänge $\lambda=254 \mathrm{~nm}$ neben der Dimerspaltung auch simultan Dimerisierungsreaktionen stattfinden [18]. Zudem kann es aufgrund der hohen Energie des Lichtes zu weiteren Nebenreaktionen kommen. Einen weiteren Einfluss hat die Eindringtiefe des Lichtes. Da nach dem LambertBeerschen-Absorptionsgesetz kurzwelligere Strahlung im Material schon nach einer kleineren Schichtdicke absorbiert wird, kann das für den Entnetzungsprozess verwendete Licht $(\lambda=254 \mathrm{~nm})$ nicht so tief in das Material eindringen, wie das zuvor zum Vernetzen vewendete Licht $(\lambda>310 \mathrm{~nm})$.

Fazit Es konnte erfolgreich die photochemische Vernetzung der Blockcopolymere P(MMA- $c$-CMA)- $b$-PBA mittels Bestrahlung von UV-Licht der Wellenlänge $\lambda>$ $310 \mathrm{~nm}$ beobachtet werden. Obwohl die Vernetzung im festen Zustand erfolgte, ist der Prozess so effektiv, dass es möglich ist Zustände einzufrieren und damit die temperaturabhängige Gleichgewichtsmorphologie zu unterdrücken. Desweiteren wurde der Einfluss die Bestrahlung beziehungsweise der Vernetzung auf die mechanischen Eigenschaften untersucht. Dabei konnte einerseits beobachtet werden, dass der Vernetzungsprozess unter mechanischer Belastung weniger effektiv ist. Im Intervallversuch hingegen zeigte der Modul einen signifikanten Anstieg mit der Betrahlungsdauer. Damit ist es möglich die Anzahl der Crosslinks über die Dauer der Bestrahlung zu variieren und zu untersuchen, ob es eine kritische Crosslinkdichte gibt, bei welcher die mikrophasenseparierte Gleichgewichtsmorphologie nicht mehr durch die Crosslinks unterdrückt werden kann. Dies ist bisher lediglich in theoretischen Modellen untersucht worden [37] und bietet ein interessantes Feld für weitere Forschung. 


\subsubsection{Ordnungs-Unordnungsübergang}

Im Hinblick auf typische Phasendiagramme von mikrophasenseparierenden Blockcopolymeren ist besonders der Übergang zum ungeordneten Zustand von Interesse. Dieser Übergang kann einerseits durch eine Temperaturerhöhung und andereseits durch einen höheren Polymerisationsgrad $N$ der Ketten erreicht werden. In dieser Arbeit wurden Blockcopolymerproben $\mathrm{P}(\mathrm{MMA}-c o-\mathrm{CMA})-b$-PBA mit verschiedener Zusammensetzung hinsichtlich ihrer Struktur und ihres thermischen Verhaltens untersucht. Da für eine Entmischung der geordneten Phase eine gewisse Mobilität der Polymerketten notwendig ist, wird der ODT oberhalb der Glasübergangstemperaturen der beiden Konstituenten erwartet. In den DMA-Messungen, konnten zwar verschiedene Relaxationsprozesse beobachtet werden, jedoch konnte zunächst kein ungeordneter Zustand mit dem AFM beobachtet werden. Dafür wurden von K.Tietz in situ Messungen mit dem AFM durchgeführt, bei denen während der Messung mit einer Heizzelle die Probentemperatur erhöht werden konnte. Dabei konnte für die Probencharge A-G der ungeordnete Zustand nicht beobachtet werden. Um einen Hinweis zu bekommen, welche Signatur ein ODT in den hier durchgeführten Experimenten zeigt, wurde zunächst ein kommerzielles Blockcopolymer PI- $b$-PS untersucht. Diese Blockcopolymer ist in der Literatur sehr bekannt und zeigt einen ODT. Die DMA-Messung zeigt im temperaturabhängigen mechanischen Verhalten neben den einzelnen Glasübergangstemperaturen der einzelenen Blöcke zusätzlich einen weiteren Übergang oberhalb der einzelnen Glasübergangstemperaturen, welcher den ODT des Systems darstellt. Der ODT ist relativ breit und im Gegensatz zu einem Glasübergang weniger deutlich ausgeprägt. Es ist jedoch zu erwarten, dass der ODT weniger Einflusss auf die mechanische Charakteristik hat, als ein Glasübergang. Da sich das System beim Übergang zum ungeordneten Zustand schon oberhalb beider Glasübergangstemperaturen befindet, sind die Polymerketten schon sehr mobil.

Demzufolge ist die Signatur des ODT im Speichermodul gering. Desweiteren kommt hinzu, dass in der vorliegenden Arbeit das stets beim Hochheizen gemessen wurde ohne einen vorangegangenen Temperprozess. Das Tempern führt jedoch dazu, dass das Polymer seine Gleichgewichtskonfiguration einnehmen kann, so dass in anschließenden DMA-Messungen Übergänge deutlicher in den mechanischen Eigenschaften $\mathrm{zu}$ beobachten sind.

Die Probe X gehört zu dem in dieser Arbeit untersuchten Blockcopolymersystem P(MMA- $c$-CMA)- $b$-PBA und weist im Unterschied zu den Proben A-G eine deutlich kleineres Molgewicht auf. Daher wird für diese Probe der ODT bei tieferen Temperaturen erwartet. Der DMA-Temperaturscan zeigt hier eindeutig neben den Glasübergängen der einzelnen Homoplymere zusätzlich einen breiten Übergang bei 471K. Dieser Übergang stellt den ODT dar, wie anhand des Vergleiches der AFMMessungen von K.Tietz festgestellt werden kann. Diese Messungen konnten mithilfe der Vernetzung des Polymers bei einer Temperatur von $453 \mathrm{~K}$ zeigen, dass das Polymer bei dieser Temperatur ungeordnet vorliegt. Die aus dem DMA-Scan bestimmte Temperatur liegt etwas höher als die Temper-Temperatur, da auch hier wieder wäh- 


\section{UV-aktive Blockcopolymere}

rend des Heizprozesses gemessen wurde. Zudem wurde der Übergang aus der Stufe im Modul bestimmt. Der Onset des ODT-Übergangs zeigt sich jedoch schon im $\tan \delta$ bei einer Temperatur von ca. 393K. Zudem ist anzunehmen ist, dass für den Prozess der Entmischung eine relativ lange Zeit benötigt wird. Die verwendete Heizrate liegt jedoch bei $2 \mathrm{~K} / \mathrm{min}$. Für eine langsamere Rate würde der Übergang analog zu Glasübergängen bei niedrigeren Temepraturen erwartet werden.

Fazit Es konnte die Signatur eines ODT in den mechanischen Eigenschaften am kommerziellen BCP PI- $b$-PS erfolgreich mit dem DMA beobachtet werden. Außerdem konnte für das in der Arbeit untersuchte BCP-System ebenfalls ein ODT beobachtet werden, indem eine Probe mit einem deutlich geringeren Molgewicht untersucht wurde. In Verbindung mit der photochemischen Vernetzung bietet der ODT ein interessantes Feld für weitergehende Untersuchungen. 


\section{Zusammenfassung und Ausblick}

In dieser Arbeit wurden dynamisch mechanische Untersuchungen an Polymeren durchgeführt. Dabei wurden einerseits eine Messmethode zur Charakterisierung des mechanischen nichtlinearen Verhaltens entwickelt. Andererseits wurde im zweiten Teil der Arbeit ein UV-aktives Blockcopolymersystem untersucht.

Es konnte erfolgreich eine Messmethode zur Untersuchung des nichtlinearen mechanischen Verhaltens entwickelt werden. Dafür wurden zwei verschiedene kommerzielle PMMA-Systeme untersucht. Es konnte eindeutig nichtlineares Verhalten detektiert werden. Dies konnte zunächst anhand von Lissajousfiguren, sowie anhand von Messungen bei verschiedenen Anregungsamplituden und Temperaturen gefunden werden. Insbesondere konnte die erwartete Abhängigkeit des Beitrages bei der drittten harmonischen Frequenz von der Anregungsamplitude gefunden werden. Desweiteren wurden Messungen durchgeführt, die eine Zeitabhängigkeit des nichtlinearen Verhaltens untersuchen. Dafür wurde ein Messablauf gewählt, bei dem die Anregungsamplitude von einem kleinen Wert auf einen großen und dann wieder auf den kleinen Anfangswert geändert wird. Bei diesen Messungen konnte beobachtet werden, dass das nichtlineare Verhalten sowohl in der fundamentalen, als auch in der dritten harmonischen Antwort eine Zeitabhängigkeit zeigt, die sich jedoch in ihrem qualitativen Verlauf unterscheiden. Im Vergleich der beiden PMMA-Systeme konnte für das $\mathrm{PMMA}^{G}$ ein Trend in den Relaxationszeiten $\tau_{K W W}$ festgestellt werden. Dazu im Gegensatz waren die Messungen am PMMA $^{E}$ im Hinblick auf die Relaxationszeiten weniger aussagekräftig, da dieses PMMA einer geringere Reinheit aufweist und daher verschieden Zusätze enthalt, die entscheidenden Einfluss auf die mechanischen Eigenschaften haben. Um genauere Informationen über die Relaxationszeiten zu erhalten, müssten mehr Messungen durchgeführt werden. Insbesondere muss dafür die Messdauer erhöht werden um eine Sättigung des beobachteten Effektes zu erreichen. Um zu ähnlichen Ergebnissen zu kommen wie Loidl et al., wäre es wünschenswert auch bei verschiedenen Frequenzen zu messen. Bisherige Messungen an PMMA konnten jedoch bisher keine brauchbaren Ergebnisse liefern, da die spektrale Auflösung des DMA8000 nicht ausreichend war, beziehungsweise bei höheren Frequenzen im verwendeten Biegebalkenexperiment Resonanzeffekte in der Anlage auftraten. Jedoch könnte in einer anderen Messgeometrie, beispielsweise in einem Doppelbiegebalkenexperiment ein breiterer Frequenzbereich experimentell zugänglich sein. In dieser Arbeit wurde die dynamisch mechanische Spektroskopie mit 


\section{Zusammenfassung und Ausblick}

großen Amplituden bisher nur an Polymersystemen eingesetzt. Es gibt daher viel Raum für neue Untersuchungen an anderen amorphen Materialien, wie beispielsweise metallischen Gläsern. Die ersten Versuche mit metallischen Gläsern sind vielversprechend und zeigen interessante Effekte die in Zukunft noch detailliert untersucht werden müssen.

Der zweite Schwerpunkt dieser Arbeit bildete die Untersuchung eines UV-aktiven Blockcopolymers. Es wurden zunächst Blockcopolymere verschiedener Zusammensetzung hinsichtlich ihres temperaturabhängigen mechanischen Verhaltens und ihrer Morphologie untersucht. Dafür wurden zudem Kleinwinkelröntgenmessungen durchgeführt. Es konnte schließlich unter Einbeziehung von AFM- Messungen von K.Tietz ein schematisches Phasendiagramm erstellt werden. Desweiteren konnten die BCPs erfolgreich durch Bestrahlung mit UV-Licht der Wellenlänge $\lambda>310 \mathrm{~nm}$ vernetzt werden. Dies zeigte sich in den mechanischen Messungen durch einen Zuwachs des Speichermoduls mit der Bestrahlungsdauer. Bei diesen Experimenten wurde zudem ein sehr interessanter Effekt gefunden. Denn die Vernetzungsreaktion zeigte unter dynamischer Dauerbelastung einen sehr geringen Zuwachs, was darauf zurückzuführen ist, dass der Vernetzungsprozess, dem eine Dimerisierungsreaktion zugrunde liegt, diffusionsgesteuert ist. Die Vernetzungsreaktion ist zudem reversibel und kann durch Bestrahlung mit Licht der Wellenlänge $\lambda=254 \mathrm{~nm}$ wieder gelöst werden. Dieser Effekt zeigte sich im Experiment durch eine Abnahme des Speichermoduls. Typischerweise zeigen einige BCP-Systeme eine Ordnungs-Unordnungsübergang bei Erhöhung der Temperatur. Zur Detektion dieses ODT wurde ein Blockcopolymer mit besonders kleiner Molmasse hergestellt und das temperaturabhängige mechanische Verhalten untersucht. Hier konnte der ODT anhand eines breiten Peakes im Verlust $\tan \delta$ gefunden werden. Dieser Übergang konnte auch von K.Tietz mit dem AFM beobachtet werden. Weiterführende Messungen könnten an dem BCP gemacht werden, welches den ODT zeigt, denn in Verbindung mit dem Vernetzen und Entnetzen könnte direkt das Wechselspiel zwischen dem Gleichgewishtzustand und den geometrischen Beschränkungen durch die Crosslinks untersucht werden. Dies ist bisher lediglich in theoretischen Arbeiten erfolgt. 


\section{Literaturverzeichnis}

[1] C.A. Angell. Structural instability and relaxation in liquid and glassy phases near the fragile liquid limit. Journal of Non-Crystalline Solids, 102(1-3):205 221, 1988. Proceedings of the Ninth University Conference on Glass Science.

[2] Svante Arrhenius. Über die reaktionsgeschwindigkeit bei der inversion von rohrzucker durch säuren. Zeitschrift für physikalische Chemie, 4:226-248, 1889 .

[3] Chiaki Azuma, Kohei Sanui, and Naoya Ogata. Synthesis and properties of photosensitive rubbers. ii. photosensitivity of cyclized polydienes and polypentenamer with pendent cinnamate groups. Journal of Applied Polymer Science, 27(6):2065-2078, 1982.

[4] Roland Böhmer, KL Ngai, CA Angell, and DJ Plazek. Nonexponential relaxations in strong and fragile glass formers. The Journal of chemical physics, 99(5):4201-4209, 1993.

[5] Frank S Bates. Polymer-polymer phase behavior. Science, 251(4996):898-905, 1991.

[6] Frank S Bates and Glenn H Fredrickson. Block copolymer thermodynamics: theory and experiment. Annual Review of Physical Chemistry, 41(1):525-557, 1990 .

[7] Th Bauer, P Lunkenheimer, and A Loidl. Cooperativity and the freezing of molecular motion at the glass transition. Physical review letters, 111(22):225702, 2013.

[8] Herbert Baur and H Baur. Thermophysics of polymers. Springer, 1999.

[9] Alexander Bergmann, Doris Orthaber, Guenther Scherf, and Otto Glatter. Improvement of saxs measurements on kratky slit systems by gobel mirrors and imaging-plate detectors. Journal of applied crystallography, 33(3):869-875, 2000 .

[10] G. Binnig, C. F. Quate, and Ch. Gerber. Atomic force microscope. Phys. Rev. Lett., 56:930-933, Mar 1986.

[11] Mahesh V Biyani, Christoph Weder, and E Johan Foster. Photoswitchable nanocomposites made from coumarin-functionalized cellulose nanocrystals. Polymer Chemistry, 5(18):5501-5508, 2014. Crosslinking im Bulk veränder modul. 
[12] Jean-Philippe Bouchaud and Giulio Biroli. Nonlinear susceptibility in glassy systems: A probe for cooperative dynamical length scales. Physical Review B, 72(6):064204, 2005.

[13] David I Bower. An introduction to polymer physics. Cambridge University Press, 2002.

[14] J Brandrup, EH Immergut, EA Grulke, A Abe, and DR Bloch. Handbook of polymers. Wiley, New York, 1989.

[15] Witold Brostow, Rachel Chiu, Ioannis M Kalogeras, and Aglaia VassilikouDova. Prediction of glass transition temperatures: binary blends and copolymers. Materials Letters, 62(17):3152-3155, 2008.

[16] C Brun, F Ladieu, D l'Hôte, M Tarzia, G Biroli, and J-P Bouchaud. Nonlinear dielectric susceptibilities: accurate determination of the growing correlation volume in a supercooled liquid. Physical Review B, 84(10):104204, 2011.

[17] B. Cappella and G. Dietler. Force-distance curves by atomic force microscopy. Surface Science Reports, 34(1-3):1 - 104, 1999.

[18] Yun Chen and Jehng-Daugh Wu. Preparation and photoreaction of copolymers derived from n-(1-phenylethyl) acrylamide and 7-acryloyloxy-4-methyl coumarin. Journal of Polymer Science Part A: Polymer Chemistry, 32(10):18671875, 1994.

[19] C Crauste-Thibierge, C Brun, F Ladieu, D L'hôte, G Biroli, and JP Bouchaud. Evidence of growing spatial correlations at the glass transition from nonlinear response experiments. Physical review letters, 104(16):165703, 2010.

[20] Pablo G Debenedetti and Frank H Stillinger. Supercooled liquids and the glass transition. Nature, 410(6825):259-267, 2001.

[21] Peter JW Debye et al. collected papers of peter jw debye. 1954.

[22] D Deng, AS Argon, and S Yip. Simulation of plastic deformation in a twodimensional atomic glass by molecular dynamics iv. Philosophical Transactions of the Royal Society of London A: Mathematical, Physical and Engineering Sciences, 329(1608):613-640, 1989.

[23] EA DiMarzio and JH Gibbs. Glass temperature of copolymers. Journal of Polymer Science, 40(136):121-131, 1959.

[24] Masao Doi and SF Edwards. Dynamics of concentrated polymer systems. part 3. - the constitutive equation. Journal of the Chemical Society, Faraday Transactions 2: Molecular and Chemical Physics, 74:1802-1832, 1978.

[25] Mi D Ediger. Spatially heterogeneous dynamics in supercooled liquids. Annual review of physical chemistry, 51(1):99-128, 2000.

[26] Paul Ehrenfest. Collected scientific papers. North-Holland Pub. Co., 1959. 
[27] Hans-Georg Elias. Makromoleküle: Physikalische Struktur $\&$ EigenschaftenSechste. John Wiley \& Sons, 2009.

[28] Stephen Richard Elliott. Physics of amorphous materials. Longman London; New York, 1983.

[29] M Fernandez-Garcia, R Cuervo-Rodriguez, and EL Madruga. Glass transition temperature of butyl acrylate-methyl methacrylate copolymers. J Polym Sci, Polym Phys, 37:2512-20, 1999.

[30] M Fernandez-Garcia, MMC Lopez-Gonzalez, JM Barrales-Rienda, EL Madruga, and C Arias. Effect of copolymer composition and conversion on the glass transition of methyl acrylate-methyl methacrylate copolymers. Journal of Polymer Science Part B: Polymer Physics, 32(7):1191-1203, 1994.

[31] Thomas G. Fox and Paul J. Flory. Second order transition temperatures and related properties of polystyrene. i. influence of molecular weight. Journal of Applied Physics, 21(6):581-591, 1950.

[32] Gordon S Fulcher. Analysis of recent measurements of the viscosity of glasses. Journal of the American Ceramic Society, 8(6):339-355, 1925.

[33] Alexander Göthlich, Sebastian Koltzenburg, and Gunnar Schornick. Funktionale polymere im alltag: Vielseitig. Chemie in unserer Zeit, 39(4):262-273, 2005 .

[34] Umesh Gaur and Bernhard Wunderlich. Study of microphase separation in block copolymers of styrene and alpha-methylstyrene in the glass transition region using quantitative thermal analysis. Macromolecules, 13(6):1618-1625, 1980 .

[35] Pierre-Giles de Gennes. Reptation of a polymer chain in the presence of fixed obstacles. Journal of chemical physics, 55:572-579, 1971.

[36] Martin Goldstein. Viscous liquids and the glass transition: a potential energy barrier picture. The Journal of Chemical Physics, 51(9):3728-3739, 1969.

[37] Enrique D Gomez, Jayajit Das, Arup K Chakraborty, John A Pople, and Nitash P Balsara. Effect of cross-linking on the structure and thermodynamics of lamellar block copolymers. Macromolecules, 39(14):4848-4859, 2006.

[38] Schmelzer J. Gutzow I. The Vitreous State. Springer, 1995.

[39] Ian W Hamley et al. The physics of block copolymers, volume 19. Oxford University Press New York, 1998.

[40] Jie He, Xia Tong, Luc Tremblay, and Yue Zhao. Corona-cross-linked polymer vesicles displaying a large and reversible temperature-responsive volume transition. Macromolecules, 42(19):7267-7270, 2009.

[41] J Heijboer and JA Prins. Physics of non-crystalline solids. JA Prins, page 231, 1965. 
[42] Andreas Heuer. Exploring the potential energy landscape of glass-forming systems: from inherent structures via metabasins to macroscopic transport. Journal of Physics: Condensed Matter, 20(37):373101, 2008.

[43] R. Hölzle. Physik der Polymere: Vorlesungsmanuskripte des 22. IFFFerienkurses vom 25.2. bis 8.3.1991 im Forschungszentrum Jülich. IFF Ferienkurs. Forschungszentrum Jülich, Institut für Festkörperforschung, 1991.

[44] Wei Huang and Ranko Richert. Dynamics of glass-forming liquids. xiii. microwave heating in slow motion. The Journal of chemical physics, 130(19):194509, 2009 .

[45] W Huang and R Richert. On the harmonic analysis of non-linear dielectric effects. The European Physical Journal B-Condensed Matter and Complex Systems, 66(2):217-221, 2008.

[46] Wei Huang and Ranko Richert. The physics of heating by time-dependent fields: microwaves and water revisited. The Journal of Physical Chemistry B, 112(32):9909-9913, 2008.

[47] Rebecca H Huyck, Scott R Trenor, Brian J Love, and Timothy E Long. Photodimerization of coumarin functionalized poly (alkyl acrylate) and poly (alkyl methacrylate) random copolymers: Influence of copolymer composition on photocrosslinking. Journal of Macromolecular Science, Part A: Pure and Applied Chemistry, 45(1):9-15, 2007.

[48] Kyu Hyun, Kyung Hyun Ahn, Seung Jong Lee, Masataka Sugimoto, and Kiyohito Koyama. Degree of branching of polypropylene measured from fouriertransform rheology. Rheologica acta, 46(1):123-129, 2006.

[49] Kyu Hyun, En Su Baik, Kyung Hyun Ahn, Seung Jong Lee, Masataka Sugimoto, and Kiyohito Koyama. Fourier-transform rheology under medium amplitude oscillatory shear for linear and branched polymer melts. Journal of Rheology (1978-present), 51(6):1319-1342, 2007.

[50] Kyu Hyun, Sook Heun Kim, Kyung Hyun Ahn, and Seung Jong Lee. Large amplitude oscillatory shear as a way to classify the complex fluids. Journal of Non-Newtonian Fluid Mechanics, 107(1):51-65, 2002.

[51] Kyu Hyun and Manfred Wilhelm. Establishing a new mechanical nonlinear coefficient q from ft-rheology: first investigation of entangled linear and comb polymer model systems. Macromolecules, 42(1):411-422, 2008.

[52] Kyu Hyun, Manfred Wilhelm, Christopher O Klein, Kwang Soo Cho, Jung Gun Nam, Kyung Hyun Ahn, Seung Jong Lee, Randy H Ewoldt, and Gareth H McKinley. A review of nonlinear oscillatory shear tests: Analysis and application of large amplitude oscillatory shear (laos). Progress in Polymer Science, 36(12):1697-1753, 2011.

[53] Y?chi Ishida, Masato Matsuo, and Kaoru Yamafuji. Dielectric behaviors 
of some polyvinyl esters. Kolloid-Zeitschrift und Zeitschrift für Polymere, 180(2):108-114, 1962.

[54] J Jackle. Models of the glass transition. Reports on Progress in Physics, 49(2):171, 1986.

[55] John David Jackson. Classical Electrodynamics. John Wiley \& Sons, Inc., 1962.

[56] Richard AL Jones. Soft condensed matter, volume 6. Oxford University Press, 2002.

[57] Ashish K Khandpur, Stephan Foerster, Frank S Bates, Ian W Hamley, Anthony J Ryan, Wim Bras, Kristoffer Almdal, and Kell Mortensen. Polyisoprene-polystyrene diblock copolymer phase diagram near the orderdisorder transition. Macromolecules, 28(26):8796-8806, 1995.

[58] Rudolf Kohlrausch. Theorie des elektrischen rückstandes in der leidener flasche. Annalen der Physik, 167(2):179-214, 1854.

[59] Dr.rer.nat. Konrad Kopitzki. Einfühtung in die Festkörperphysik. Teubner Studienbücher, 2 edition, 1989.

[60] Daniel J Lacks. Energy landscapes and the non-newtonian viscosity of liquids and glasses. Physical review letters, 87(22):225502, 2001.

[61] Marc Langela. Struktur und rheologische Eigenschaften von PS-PI- und PS$P B$-Blockcopolymeren. PhD thesis, Johannes-Gutenberg-Universität Mainz, 2001.

[62] Manfred D Lechner, Klaus Gehrke, Eckhard H Nordmeier, and U Guhr. Makromolekulare chemie. Springer, 1993.

[63] JL Lustres, Vadim M Farztdinov, and Sergey A Kovalenko. Femtosecond s3 -> s1 conversion and structural reorganisation of trans-3-phenylprop-2enaldehyde and derivatives in solution. ChemPhys Chem, 6(8):1590-1599, 2005.

[64] MW Matsen and Frank S Bates. Unifying weak-and strong-segregation block copolymer theories. Macromolecules, 29(4):1091-1098, 1996.

[65] PH Mott, AS Argon, and UW Suter. Atomistic modelling of plastic deformation of glassy polymers. Philosophical Magazine A, 67(4):931-978, 1993.

[66] E Muzeau, J Perez, and GP Johari. Mechanical spectrometry of the. beta.relaxation in poly (methyl methacrylate). Macromolecules, 24(16):4713-4723, 1991.

[67] Alexandre Nicolas, Kirsten Martens, and Jean-Louis Barrat. Rheology of athermal amorphous solids: Revisiting simplified scenarios and the concept of mechanical noise temperature. EPL (Europhysics Letters), 107(4):44003, 2014. 
[68] Allen C Pipkin. Lectures on viscoelasticity theory, volume 7. Springer Science \& Business Media, 2012.

[69] Donald J Plazek and Kia L Ngai. Correlation of polymer segmental chain dynamics with temperature-dependent time-scale shifts. Macromolecules, 24(5):1222-1224, 1991.

[70] T. PRITZ. Verification of local kramers-kronig relations for complex modulus by means of fractional derivative model. Journal of Sound and Vibration, 228(5):1145 - 1165, 1999.

[71] Alexander Rahn. Thermische und mechanische analyse von diblockcopolymeren. Master's thesis, Georg-August-Universität, 2012.

[72] Ranko Richert. Frequency dependence of dielectric saturation. Physical Review E, 88(6):062313, 2013.

[73] Ranko Richert. Calorimetry based on energy absorbed from time-dependent fields. Journal of Non-Crystalline Solids, 357(2):726-730, 2011.

[74] R. Richert. Confinement effects in bulk supercooled liquids. The European Physical Journal Special Topics, 189(1):223-229, 2010.

[75] Ranko Richert. Heterogeneous dynamics in liquids: fluctuations in space and time. Journal of Physics: Condensed Matter, 14(23):R703, 2002.

[76] R Richert and K Samwer. Enhanced diffusivity in supercooled liquids. New Journal of Physics, 9(2):36, 2007.

[77] Ranko Richert and Susan Weinstein. Nonlinear dielectric response and thermodynamic heterogeneity in liquids. Physical review letters, 97(9):095703, 2006.

[78] Prince E Rouse Jr. A theory of the linear viscoelastic properties of dilute solutions of coiling polymers. The Journal of Chemical Physics, 21(7):12721280, 1953.

[79] Srikanth Sastry, Pablo G Debenedetti, and Frank H Stillinger. Signatures of distinct dynamical regimes in the energy landscape of a glass-forming liquid. Nature, 393(6685):554-557, 1998.

[80] Felix H Schacher, Paul A Rupar, and Ian Manners. Functional block copolymers: nanostructured materials with emerging applications. Angewandte Chemie International Edition, 51(32):7898-7921, 2012.

[81] B Schiener, R Böhmer, A Loidl, and RV Chamberlin. Nonresonant spectral hole burning in the slow dielectric response of supercooled liquids. Science, 274(5288):752-754, 1996.

[82] B Schiener, RV Chamberlin, G Diezemann, and R Böhmer. Nonresonant dielectric hole burning spectroscopy of supercooled liquids. The Journal of chemical physics, 107(19):7746-7761, 1997. 
[83] GMJ Schmidt. Photodimerization in the solid state. Pure and Applied Chemistry, 27(4):647-678, 1971.

[84] Hans Sillescu. Heterogeneity at the glass transition: a review. Journal of Non-Crystalline Solids, 243(2):81-108, 1999.

[85] Hans W Spiess. Molecular dynamics of solid polymers as revealed by deuteron nmr. Colloid and Polymer Science, 261(3):193-209, 1983.

[86] Wilasinee Sriprom, Michael James, Se?bastien Perrier, and Chiara Neto. Ordered microphase separation in thin films of pmma- pba synthesized by raft: Effect of block polydispersity. Macromolecules, 42(8):3138-3146, 2009.

[87] Herrmann Staudinger. Die chemie der hochmolekularen organischen stoffe im sinne der kekuléschen strukturlehre. Berichte der deutschen chemischen Gesellschaft (A and B Series), 59(12):3019-3043, 1926.

[88] Hermann Staudinger. Über polymerisation. Berichte der deutschen chemischen Gesellschaft (A and B Series), 53(6):1073-1085, 1920.

[89] Gert R Strobl and Gert R Strobl. The physics of polymers, volume 2. Springer, 1997.

[90] Gf Tammann and W Hesse. Die abhängigkeit der viscosität von der temperatur bie unterkühlten flüssigkeiten. Zeitschrift für anorganische und allgemeine Chemie, 156(1):245-257, 1926.

[91] C Thibierge, D l'Hôte, F Ladieu, and R Tourbot. A method for measuring the nonlinear response in dielectric spectroscopy through third harmonics detection. Review of Scientific Instruments, 79(10):103905, 2008.

[92] Bernd Tieke. Makromolekulare Chemie: Eine Einführung. John Wiley \& Sons, 2014.

[93] K. Tietz. Mikrophasenseparation von photo-vernetzbaren Blockcopolymeren in dünnen Filmen. PhD thesis, Georg-August-Universität Göttingen, 2014.

[94] Katharina Tietz, Stefanie Finkhäuser, Konrad Samwer, and Philipp Vana. Stabilizing the microphase separation of block copolymers by controlled photocrosslinking. Macromolecular Chemistry and Physics, 215(16):1563-1572, 2014.

[95] Marcel Utz, Pablo G Debenedetti, and Frank H Stillinger. Atomistic simulation of aging and rejuvenation in glasses. Physical review letters, 84(7):1471, 2000 .

[96] Hans Vogel. Das temperaturabhängigkeitsgesetz der viskosität von flüssigkeiten. Phys. Z, 22:645-646, 1921.

[97] Li-Min Wang and Ranko Richert. Measuring the configurational heat capacity of liquids. Physical review letters, 99(18):185701, 2007. 
[98] Q Wang, ST Zhang, Y Yang, YD Dong, CT Liu, and J Lu. Unusual fast secondary relaxation in metallic glass. Nature communications, 6, 2015.

[99] Graham Williams and David C Watts. Non-symmetrical dielectric relaxation behaviour arising from a simple empirical decay function. Transactions of the Faraday society, 66:80-85, 1970.

[100] Malcolm L Williams, Robert F Landel, and John D Ferry. The temperature dependence of relaxation mechanisms in amorphous polymers and other glassforming liquids. Journal of the American Chemical society, 77(14):3701-3707, 1955 .

[101] A. Willmes. Taschenbuch chemische Substanzen: Elemente - Anorganika Organika - Naturstoffe - Polymere. Deutsch, 2007.

[102] Warren Clarence Young and Richard Gordon Budynas. Roark's formulas for stress and strain, volume 7. McGraw-Hill New York, 2002.

[103] Hai-Bin Yu, Ranko Richert, Robert Maaß, and Konrad Samwer. Strain induced fragility transition in metallic glass. Nature communications, 6, 2015.

[104] Jerzy Zarzycki. Glasses and the vitreous state. Number 9. Cambridge University Press, 1991.

[105] Jin Zhao, Biswaroop Majumdar, Mark F Schulz, Frank S Bates, Kristoffer Almdal, Kell Mortensen, Damian A Hajduk, and Sol M Gruner. Phase behavior of pure diblocks and binary diblock blends of poly (ethylene)-poly (ethylethylene). Macromolecules, 29(4):1204-1215, 1996.

[106] Bruno H Zimm. Dynamics of polymer molecules in dilute solution: viscoelasticity, flow birefringence and dielectric loss. The journal of chemical physics, 24(2):269-278, 1956.

[107] Perkin Elmer. DMA 8000 Manual. 


\section{Abbildungsverzeichnis}

2.1. Schematisch dargestellte Temperaturabhängigkeit (a) des Volumens eines Glasbildners, (b) der spezifischen Wärme eines Glases. Nach [28] 11

2.2. Angell-Plot: Temperaturabhängigkeit der Viskosität oberhalb der Glastemperatur für verschiedene Gläser [1]. . . . . . . . . . . . . . 14

2.3. Frequenzabhängigkeit des dielektrischen Verlustes $\epsilon$ " aus [54]. Die Messung stammt von Ishida et al. [53]. . . . . . . . . . . . 16

2.4. Schematische Darstellung der PEL für ein starkes und ein fragiles Glas (aus [20]) ...................... 17

2.5. Schematische Darstellung des Einflusses von mechanischer Dehung auf eine Energiebarriere. $[60] \ldots \ldots \ldots \ldots 17$

2.6. Einfache Modelle zur Beschreibung viskoelastischen Verhaltens . . . . 20

2.7. Der Phasenwinkel zwischen Anregung und Antwort ist ein Maß für den Verlust im Material. . . . . . . . . . . . . . . 22

2.8. Monomereinheit von Polymethylmethacrylate (PMMA) . . . . . . . 22

2.9. Temperaturabhängigkeit des Verlustmoduls für verschiedene Polyme-

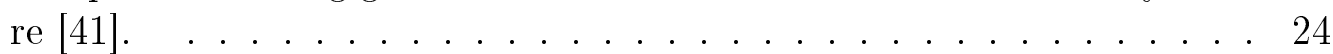

2.10. Schema der Reptation eines Poylmers [56] . . . . . . . . . . 24

2.11. Ein Diblockcopolymer kann nur auf Längenskalen entsprechend der Polymerlänge entmischen. Mit steigender Temperatur wird die Entropie erhöht, so dass es zu einem ungeordneten Zustand kommt. Nach

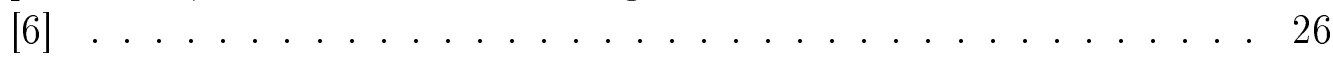

2.12. Theoretisches Phasendiagramm für ein symmetrisches Blockcopolymer nach [64]. . . . . . . . . . . . . . . . . . 26

2.13. Mechanisches Verhalten von Materialien in Abhängigkeit der Deborahzahl und der Spannungsamplitude. [68] . . . . . . . . . . . . 28

2.14. Darstellung des Prinzips des dielektrischen Lochbrennens zur Unterscheidung eines homogenen und eines heterogenen Szenarios als Ursache für das Absorptionsspektrum [81]. . . . . . . . . . . 32

2.15. Schematische Darstellung der spektralen (links) und der räumlichen (rechts) Verteilung der Relaxationszeiten in einem System mit hetereogener Dynamik. Die Dispersion wird durch 5 unterschiedliche Zeitkonstanten beschrieben $\tau_{1}$ bis $\tau_{5}$ Die räumliche Darstellung verdeutlicht, dass eine relativ schnelle Mode wahrscheinlich von viel langsameren Moden begrenzt ist. [74] ............ . . 33 
2.16. Spektrum für die dielektrische Suszeptibilität dritter Ordnung aufgetragen als $\left|\chi_{3} E^{2}\right|$ für verschiedene Temperaturen. Das Inset zeigt die Amplitude des Maximums von $\chi_{3}$, welches proportional zu $N_{\text {corr }}$ ist [7]. 34

3.1. Schematischer Aufbau der Blockcopolymere . . . . . . . . . . . 36

3.2. Schematischer Aufbau der DMA8000 (nach [107]) . . . . . . . . . 38

3.3. Messgeometrie im Zugversuch . . . . . . . . . . . . . . . . . 39

3.4. Messgeometrie im Biegebalkenexperiment . . . . . . . . . . . . 39

3.5. Temperaturprotokoll für die Charakterisierung der Blockcopolymere. Die Probe wird von Raumtemperatur mit einer Rate von $2 \mathrm{~K} / \mathrm{min}$ auf 193K gekühlt, nach 5 min Haltezeit wird das Material mit $2 \mathrm{~K} / \mathrm{min}$ auf 523K erhitzt, und anschließend auf Raumtemperatur gekühlt. . . 41

3.6. Bestimmung von Tg über den Wendepunkt im Modul mittels der Tangentenmethode, beispielhaft gezeigt an der Abkühlkurve der Probe 28(PMMA-co-Coumarin). . . . . . . . . . . . 42

3.7. Typischer Zeitverlauf einer Messung. Der obere Graph zeigt die Kraftanregung und der untere zeigt eine typische Antwortfunktion, die für jede Periode fouriertransformiert wurde. . . . . . . . . . 44

3.8. Funktionsprinzip des AFM. Schematisch dargestellt. . . . . . . . . . 46

4.1. Spannungs-Dehnungsdiagramm für $\mathrm{PMMA}^{E}$ gemessen in Biegebalkengeometrie bei verschiedenen Temperaturen. Die Kraft wurde mit einer Rate von $0,5 \mathrm{~N} / \mathrm{min}$ bis auf einen Maximalwert von $10 \mathrm{~N}$ erhöht. $\quad 50$

4.2. Temperaturscans von $\mathrm{PMMA}^{E}$ und $\mathrm{PMMA}^{G} \ldots \ldots \ldots \ldots$. . . . . 51

4.3. Lissajousfiguren für eine $\mathrm{PMMA}^{E}$-Probe im Biegebalkenversuch bei $353 \mathrm{~K}$ für verschiedene dynamische Kraftamplituden bei einer Tem-

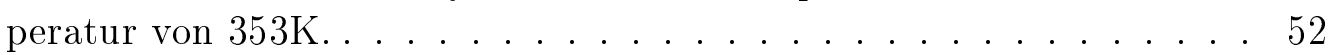

4.4. Spannungssignal und Dehnungssantwort (linke Seite) und die daraus

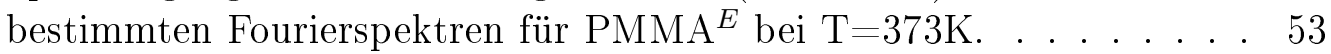

4.5. Verhältnis der Amplituden der Dehnungsantwort bei $\omega$ und $3 \omega$, aufgetragen gegen das Quadrat der Spannungsamplitude für verschiedene

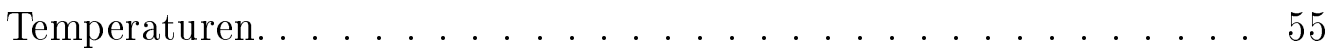

4.6. Steigung b der Geraden $A_{3 \omega} / A_{1 \omega}\left(\sigma_{0}\right)=a+b \sigma_{0}^{2} \ldots \ldots . . . . . \quad 55$

4.7. Die Auftragung von $\ln \left(b-b_{0}\right)$ in Abhängigkeit der Temperatur zeigt einen linearen Zusammenhang mit der Steigung 0,24. . . . . . . . . 56

4.8. Amplituden der ungeraden Harmonischen der Probenantwort in Abhängigket der Spannungsamplitude für $\mathrm{PMMA}^{E}$ bei einer Temperatur

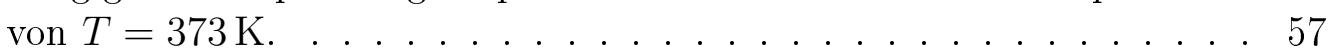

4.9. Fouriertransformierte Auslenkungsamplitude einer PMMA-Probe bei $373 \mathrm{~K}$ für verschiedene harmonische Frequenzen $i \omega$, der Anregungsfrequenz $\omega=1 \mathrm{~Hz}$. Die Kraftamplitude im niedrigen Feldbereich betrug $0,5 \mathrm{~N}(\approx 0,8 \mathrm{MPa})$ und im Hochfeldbereich $3 \mathrm{~N}(\approx 5 \mathrm{MPa}) . \ldots . .58$ 
4.10. Erste harmonische Dehnungsamplitude $\epsilon_{1 \omega}^{*}$ für verschiedene Temperaturen für PMMA ${ }^{E}$. Die Anregungsspannung im Niedrigfeldbereich betrug jeweils $\sigma_{0}=0,8 \mathrm{MPa}$ und im Hochfeldbereich $\sigma_{0}=5 \mathrm{MPa}$. Die verwendete Frequenz betrug $1 \mathrm{~Hz} . \ldots \ldots . \ldots 59$

4.11. Komplexe Dehnungsamplitude $\epsilon_{3 \omega}^{*}$ für verschiedene Temperaturen für PMMA $^{E}$. Die Anregungsspannung im Niedrigfeldbereich betrug jeweils $\sigma_{0}=0,8 \mathrm{MPa}$ und im Hochfeldbereich $\sigma_{0}=5 \mathrm{MPa}$. Die verwendete Frequenz betrug $1 \mathrm{~Hz}$. . . . . . . . . . . . 59

4.12. a)Plateauwerte für die komplexe Dehnungsamplituden bei der fundamentalen und der dritten Harmonischen der Anregungsfrequenz $\epsilon_{1 \omega}^{*}$ und $\epsilon_{3 \omega}^{*}$ für $\mathrm{PMMA}^{E}$. Das Inset zeigt die Temperaturabhängigkeit des Verhältnisses $\epsilon_{3 \omega}^{*} / \epsilon_{1 \omega}^{*}$ b) Speichermodul und Verlust bestimmt aus den Plateauwerten von $\epsilon_{1 \omega}^{*}$. Die gezeigten Werte wurden aus den letzten 100 Perioden des Hochfeldbereiches bestimmt. In diesem Bereich hatte die Anregung eine Spannungsamplitude von $5 \mathrm{MPa}$ bei einer Frequenz von $\omega=1 \mathrm{~Hz} . \ldots \ldots \ldots 60 \ldots \ldots$

4.13. Dehnungsantwort bei der fundamentalen Frequenz $1 \mathrm{~Hz}, \epsilon_{1 \omega}^{*}$ für verschiedene dynamische Kraftamplituden bei $\mathrm{T}=373 \mathrm{~K}$ für $\mathrm{PMMA}^{E} \ldots$. . 61

4.14. Antwort der Dehnung bei der 3. Harmonischen der Anregungsfrequenz $\epsilon_{3 \omega}^{*}$ für verschiedene dynamische Kraftamplituden bei $\mathrm{T}=373$ $\mathrm{K}_{\text {für } \mathrm{PMMA}^{E} \ldots \ldots \ldots \ldots \ldots \ldots 6 . \ldots \ldots \ldots}$

4.15. Plateauwerte für den Hochfeldbereich für $\mathrm{PMMA}^{E}$ für verschiedene Anregungsamplituden bei einer Temperatur von 373K (10K unterhalb von $T_{g}$.) a)Komplexe Dehnungsamplituden bei der fundamentalen und der dritten harmonischen der Anregungsfrequenz $\epsilon_{1 \omega}^{*}$ und $\epsilon_{3 \omega}^{*}$ b) Speichermodul und Verlust. . . . . . . . . . . . . . 63

4.16. Erste harmonische Dehnungsamplitude $\epsilon_{1 \omega}^{*}$ für verschiedene Temperaturen für PMMA ${ }^{G}$. Die Anregungsspannung im Niedrigfeldbereich betrug jeweils $\sigma_{0}=2,8 \mathrm{MPa}$ und im Hochfeldbereich $\sigma_{0}=16,8 \mathrm{MPa}$. Die verwendete Frequenz ist $1 \mathrm{~Hz} . \ldots \ldots$. . . . . . . 64

4.17. Dritte harmonische Dehnungsamplitude $\epsilon_{3 \omega}^{*}$ für verschiedene Temperaturen für PMMA ${ }^{G}$. Die Anregungsspannung im Niedrigfeldbereich betrug jeweils $\sigma_{0}=2,8 \mathrm{MPa}$ und im Hochfeldbereich $\sigma_{0}=16,8 \mathrm{MPa}$. Die verwendete Frequenz ist $1 \mathrm{~Hz} . \ldots \ldots$. . . . . . . . 64

4.18. Temperaturabhängigkeit der Plateauwerte für den Hochfeldbereich für $\mathrm{PMMA}^{G}$. a) Komplexe Dehnungsamplituden bei der fundamentalen und der dritten Harmonischen der Anregungsfrequenz $\epsilon_{1 \omega}^{*}$ und $\epsilon_{3 \omega}^{*}$. Das Inset zeigt die Temperaturabhängigkeit des Verhältnisses $\epsilon_{3 \omega}^{*} / \epsilon_{1 \omega}^{*}$ b) Speichermodul und Verlust. Die gezeigten Werte wurden aus den letzten 100 Perioden des Hochfeldbereiches bestimmt. In diesem Bereich hatte die Anregung eine Spannungsamplitude von 16, 8 MPa bei einer Frequenz von $\omega=1 \mathrm{~Hz} . \ldots \ldots \ldots . \ldots . \ldots 65$ 
4.19. Erste harmonische Antwort-Amplitude $\epsilon_{1 \omega}^{*}$ für verschiedene dynamische Spannungen im Hochfeldbereich für PMMA ${ }^{G}$ bei $\mathrm{T}=373 \mathrm{~K}$. Die Spannungsamplitude in den Niedrigfeldbereichen beträgt $2,8 \mathrm{MPa}$ und es wurde eine Frequenz von $1 \mathrm{~Hz}$ verwendet. . . . . . . . . . . 66

4.20. Dritte harmonische Antwort-Amplitude $\epsilon_{3 \omega}^{*}$ für verschiedene dynamische Spannungen im Hochfeldbereich für $\mathrm{PMMA}^{G}$ bei $\mathrm{T}=373 \mathrm{~K}$. Die Spannungsamplitude in den Niedrigfeldbereichen beträgt 2,8 MPa und es wurde eine Frequenz von $1 \mathrm{~Hz}$ verwendet. . . . . . . . . . 66

4.21. Plateauwerte für den Hochfeldbereich für $\mathrm{PMMA}^{G}$ in für verschiedene Spannungsamplituden bei einer Temperatur von 378 K. . . . . . . . . 67

4.22. Komplexe Nachgiebigkeiten $C_{3}^{*(1)}$ und $C_{3}^{*(3)}$ normiert auf die fundamentale Nachgiebigkeit im kleinen Feld für PMMA ${ }^{G}$ bei $T=378 \mathrm{~K} . \quad$ • 68

4.23. Relaxationszeit $\tau_{K W W}$ bestimmt aus der Zeitabhängigkeit der fundamentalen und der 3.harmonischen Dehnungsantwort im Hochfeldbereich für PMMA ${ }^{E}$ bei (a) verschiedenen Temperaturen und (b) verschiedenen Spannungsamplituden $\sigma_{0} \ldots \ldots \ldots \ldots$. . . . . . . 69

4.24. Relaxationszeit $\tau_{K W W}$ bestimmt aus der Zeitabhängigkeit der fundamentalen und der 3. harmonischen Dehnungsantwort im Hochfeldbereich für $\mathrm{PMMA}^{G}$ bei (a) verschiedenen Temperaturen und (b) verschiedenen Spannungsamplituden $\sigma_{0} \ldots \ldots \ldots$. . . . . . . . 69

5.1. Thermogramme für BCP-Proben mit verschiednem BA-Anteil. Es sind Massenverlust (rot) DTA-Signal (schwarz) und Basislinie des DTA-Signals (grau) eingezeichnet. . . . . . . . . . . . 82

5.2. Speichermodul und $\tan (\delta)$ des reinen Butylacrylates in Abhängigkeit der Temperatur, gemessen während des Aufheizens. Die bestimmten Glastemperaturen sind in rot eingezeichnet. . . . . . . . . . . 83

5.3. Temperaturabhängigkeit des Modul und des $\tan (\delta)$ von RAFT synthetisiertem PMMA. . . . . . . . . . . . . . 84

5.4. Peak $i m \tan \delta$ gemessen während des Abkühlens für die PMMA-coCMA-Blöcke. .................. 85

5.5. Übersicht der Glasübergangstemperaturen der untersuchten Blockcopoymere ..................... 86

5.6. Speichermodul und $\tan \delta$ der Probe $\mathrm{D}(37-08)$. Diese Probe zeigte bei AFM Messungen die Struktur von perforierten Lamellen [93]. . . . . . 87

5.7. Speichermodul und $\tan \delta$ der Probe E(37-10). Diese Probe zeigte bei AFM-Messungen die Struktur von perforierten Lamellen [93]. . . . . 88

5.8. Speichermodul und $\tan \delta$ der Probe F(30-05). Diese Probe zeigte bei AFM Messungen die Struktur von liegenden PMMA Zylindern [93]. • 88

5.9. Speichermodul und $\tan \delta$ der Probe G(47-01). Diese Probe zeigte im AFM die Struktur von hexagonal geordneten Sphären [93]. . . . . . . 89

5.10. Speichermodul und $\tan \delta$ der Probe H47-03). Diese Probe zeigte im AFM die Struktur von hexagonal geordneten Sphären [93]. . . . . . . 90 
5.11. Röntgen-Spektren, für Proben mit verschiedenem BA-Gehalt. Es ist der Logarithmus der gemessenen Intensität in Abhängigkeit des Streuvektors $q$ aufgetragen. . . . . . . . . . . . . . 91

5.12. a)UV-Vis-Spektrum der Probe E(37-10) nach Bestrahlung mit UVLicht der Wellenlänge $\lambda<310 \mathrm{~nm}$ für verschiedene Bestrahlungsdauern $t$; b) zeitabhängige Entwicklung der Absorptionsbande bei $317 \mathrm{~nm}$

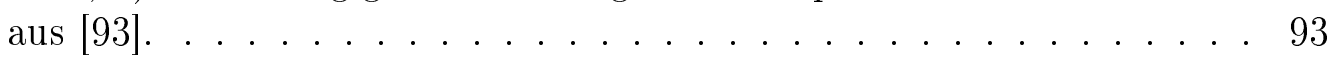

5.13. DMA Temperaturscans einer bestrahlten und einer unbestrahlten Probe $\mathrm{F}(30-5) 70 \%$ BA. . . . . . . . . . . . . 93

5.14. Speichermodul in Abhängigkeit der Zeit für die Probe 37-08. Es wurde jeweils eine Probe während der Messung dauerhaft mit UV-Licht $\lambda<$ $310 \mathrm{~nm}$ bestrahlt und die andere nicht. . . . . . . . . . . . . . 94

5.15. Modul in Abhängigkeit der Dauer der Bestrahlung mit Licht der Wellenlänge $\lambda>310 \mathrm{~nm}$. Während der Bestrahlung wurden die Proben nicht dynamisch beansprucht. . . . . . . . . . . . 95

5.16. Abhängigkeit des Moduls von der Dauer der Bestrahlung mit $\lambda=$

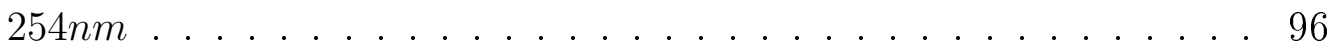

5.17. Temperaturscan des PSPI-Blockcopolymers. Es sind der $\tan \delta$ und Modul aufgetragen. Das Inset zeigt einen vergrößerten Auschnitt für

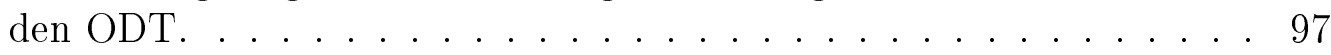

5.18. DMA-Temperaturscan der Probe X. Es sind der temperaturabhängige $\tan \delta$ und der Modul dargestellt. . . . . . . . . . . . . 98

5.19. AFM-Höhenprofile des BCPS X (70 mol \% BA) gemessen bei $294 \mathrm{~K}$ : a1) Ausgangsprobe Xa b1) Ausgangaprobe Xb a2) Probe Xa nach 4h Bestrahlung mit $(\lambda<310 \mathrm{~nm})$ bei $453 \mathrm{~K}$ b2) Probe Xb nach $4 \mathrm{~h}$ Tempern bei $453 \mathrm{~K}$ aus $[93] \ldots \ldots \ldots . \ldots . \ldots 99$

5.20. Vergrößerter Ausschnitt aus dem DMA-Temperaturscan der Probe

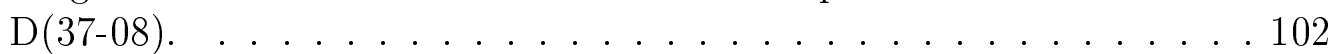

5.21. AFM-Aufnahme der Topographie der Probe G(37-8) a) vor dem Tempern bei 433K b) nach dem Tempern. . . . . . . . . . . . . . 103

5.22. Topographien der Proben a) E (37-10) (61\%BA) und b) F(30-05)(68\%BA) 104

5.23. Morphologien für verschiedene Zusammensetzungen des BCPs P(MMA$c o$-CMA)-b-BA. Die gestrichelt gekennzeichneten Morphologien sind von Sriprom et. al aus[86] entnommen. . . . . . . . . . 105

A.1. Verlauf der Temperatur und der komplexen Dehnungsamplitude $\epsilon_{1 \omega} *$ für PMMA ${ }^{E}$ im Hochfeldbereich $(\mathrm{t}=1000-2000 \mathrm{~s})$ bei einer Spannungsamplitude von $\sigma_{0}=3,2 \mathrm{MPa} . \ldots \ldots \ldots 127 \ldots \ldots$. . . . . . . . . . . .

A.2. Zeitlicher Temperaturverlauf während Messungen mit einer Spannungsamplitude von $\sigma_{0}=0,8 M P a$ im Niedrigfeldbereich(t $=0-1000$ und $\mathrm{t}=2000-3000)$ für verschiedene $\sigma_{0}$ im Hochfeldbereich. Die Spannunsamplitude im Hochfeld ist jeweils angegeben. . . . . . . . 128 
Abbildungsverzeichnis

A.3. Temperaturabhängiger Modul und $\tan \delta$ des reinen Coumarins. Bestimmt mir dem DMA8000 mit einer Frequenz von $5 \mathrm{~Hz}$ und einer Aulenkung von $0,02 \mathrm{~mm}$. Es wurde während des Heizens gemessen. . 129 


\section{A. Anhang}

\section{Temperaturverlauf während der nichtlinearen Messungen}

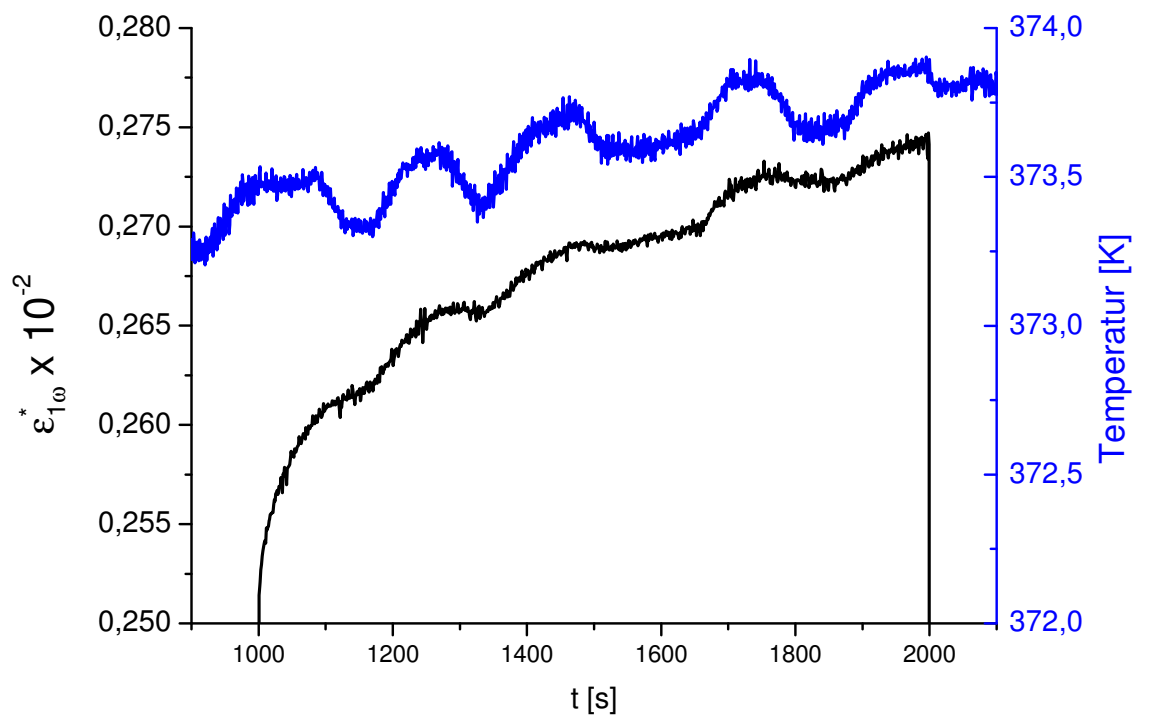

Abb. A.1.: Verlauf der Temperatur und der komplexen Dehnungsamplitude $\epsilon_{1 \omega *} *$ für $P M M A^{E}$ im Hochfeldbereich ( $t=1000$-2000s) bei einer Spannungsamplitude von $\sigma_{0}=$ 3, $2 \mathrm{MPa}$. 


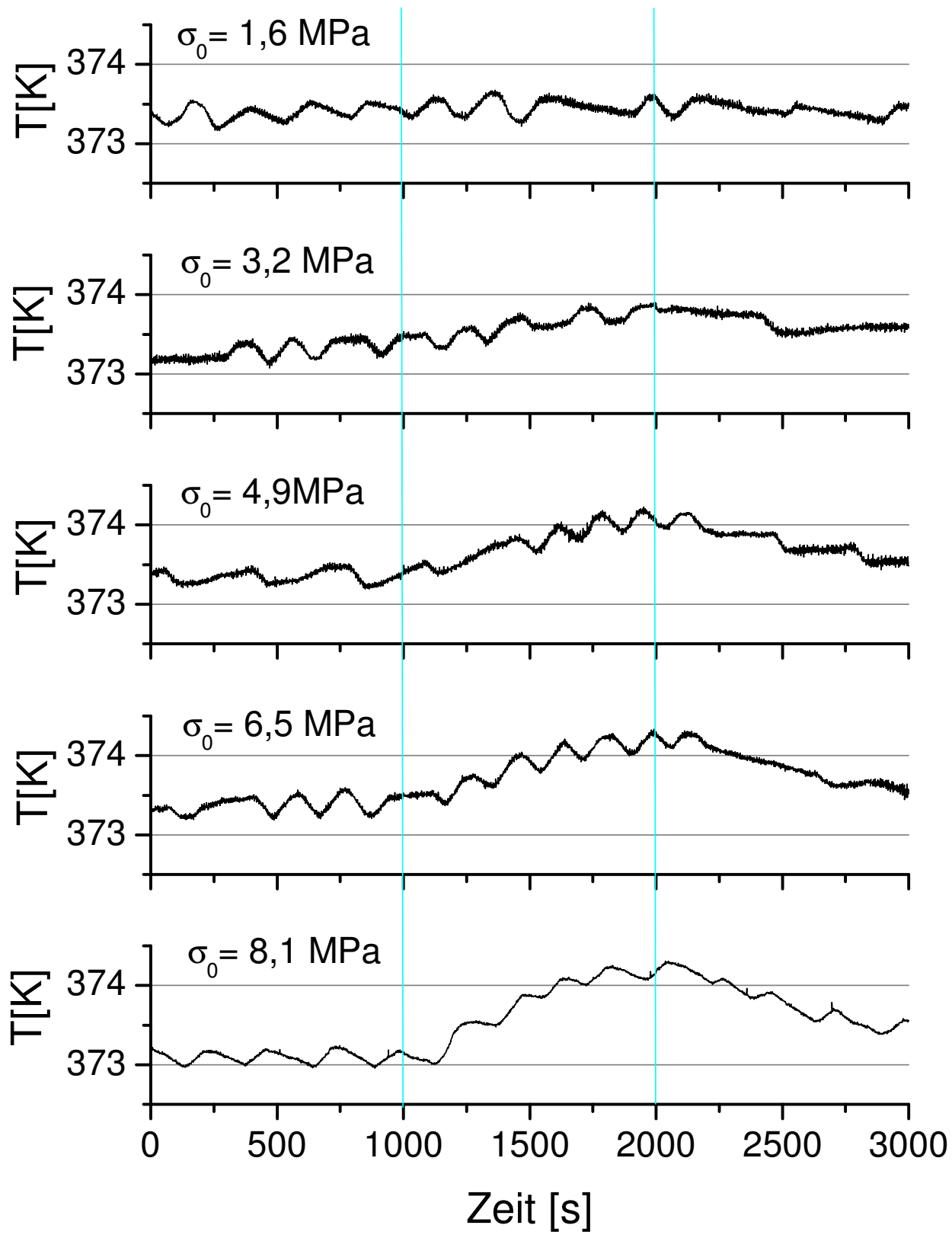

Abb. A.2.: Zeitlicher Temperaturverlauf während Messungen mit einer Spannungsamplitude von $\sigma_{0}=0,8 \mathrm{MPa}$ im Niedrigfeldbereich( $t=0$-1000 und $t=2000$-3000) für verschiedene $\sigma_{0}$ im Hochfeldbereich. Die Spannunsamplitude im Hochfeld ist jeweils angegeben. 


\section{Temperaturscan für CMA}

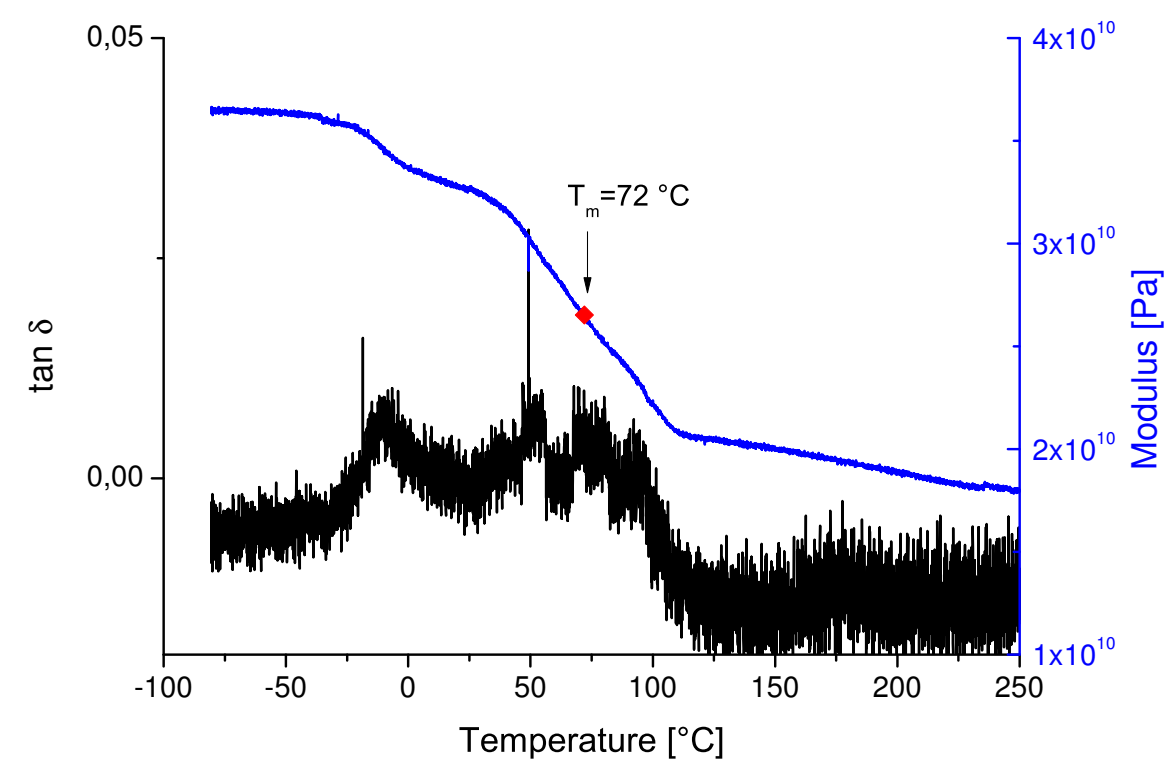

Abb. A.3.: Temperaturabhängiger Modul und tan $\delta$ des reinen Coumarins. Bestimmt mir dem DMA8000 mit einer Frequenz von 5Hz und einer Aulenkung von 0,02mm. Es wurde während des Heizens gemessen.

\section{Skript}

Skript zur Berechnung der FFTS und Bestimmung der Peakhöhen der 1. und 3.Harmonischen.

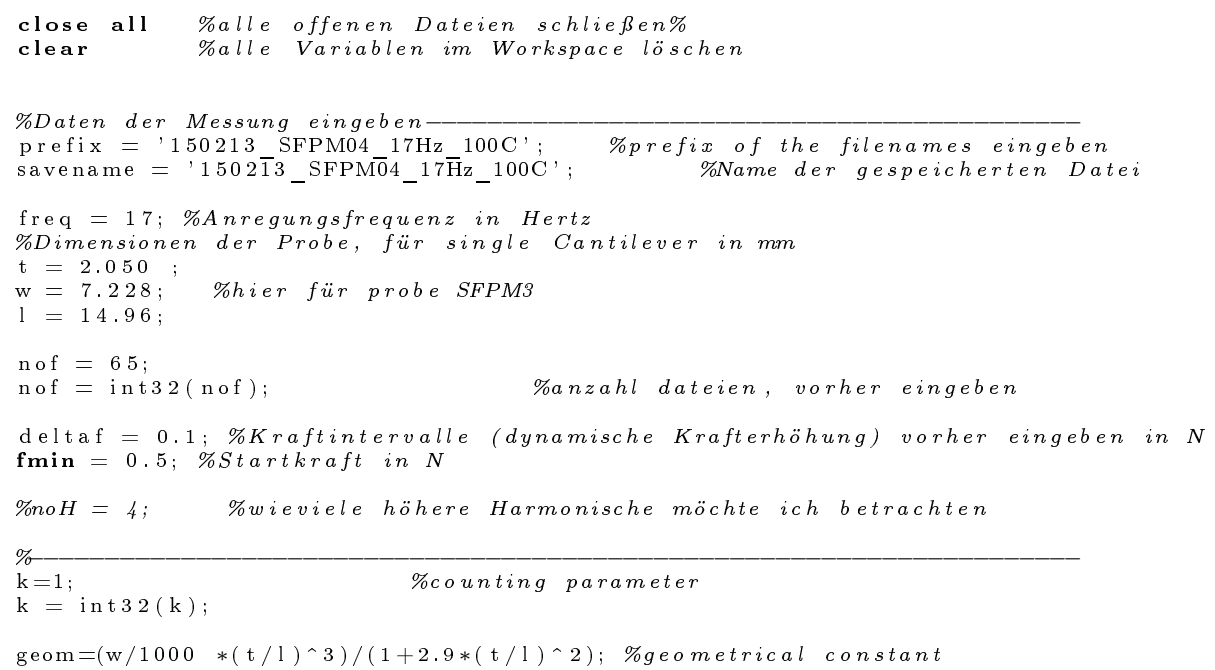




\section{A. Anhang}

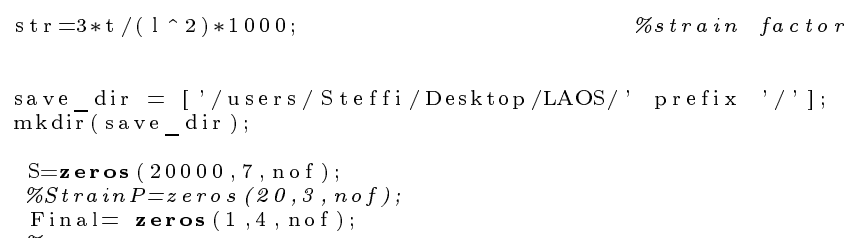




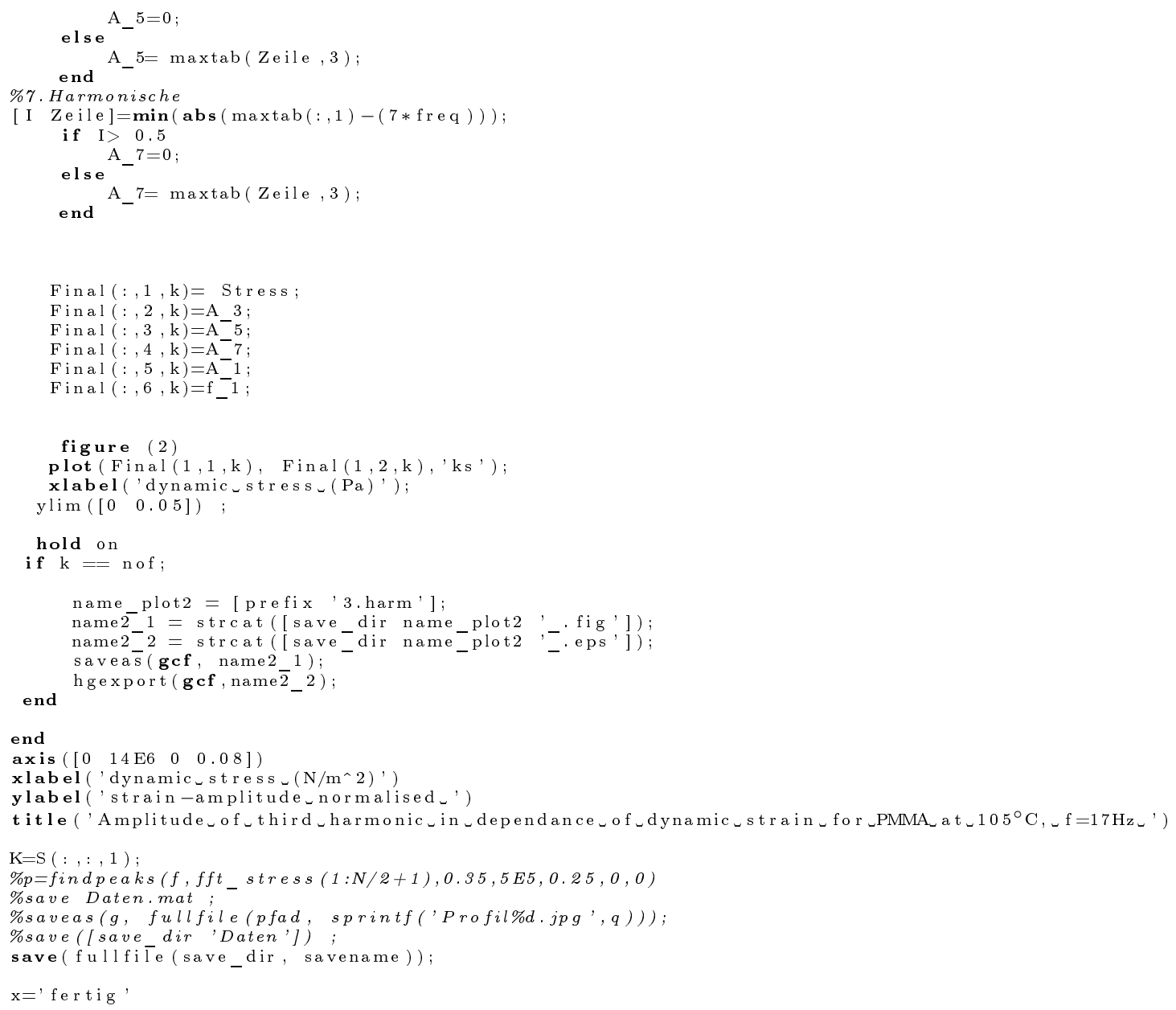





\section{B. Abkürzungen}

AFM Rasterkraftmikroskop (engl. Atomic Force Microscope)

BA $n$-Butylacrylat

BCP Blockcopolymer

CMA 7-(2-Methacryloyloxyethoxy)-4-methylcoumarin

DMA Dynamisch-Mechanischer Analysator

DTA Differenz-Thermoanalyse

et al. et alii (und andere)

KWW Kohlrausch-William-Watts

MMA Methylmethacrylat

MPS Mikrophasenseparation

NHN Niedrigfeld-Hochfeld-Niedrigfeld (Messprotokoll für nichtlineare Messungen)

ODT Ordnungs-Unordnungs-Übergang (engl.: order-disorder transition)

PBA Polybutylacrylat

PEL Potentielle Energielandschaft (engl. Potential Energy Landscape)

PMMA Polmethylmethacrylat

$\mathrm{PMMA}^{G} \quad$ Polmethylmethacrylat von Goodfellow (hohe Reinheit)

$\mathrm{PMMA}^{E} \quad$ Polmethylmethacrylat von Evonik (geringere Reinheit)

QNM Quantitative Nanomechanical Mapping

RAFT Reversibler Additions-Fragmentierungs-Kettentransfer

SAXS Kleinwinkelröntgenstreuung (engl. Small-angle X-ray scattering)

TGA Thermogravimetrische Analyse

$\mathrm{UV} / \mathrm{Vis} \quad$ Ultraviolett/ sichtbar (engl. visible)

VFT Vogel-Fulcher-Tammann 



\section{Danksagung}

An dieser Stelle möcht ich mich gerne bei denjenigen bedanken, die mich auf dem Weg diese Arbeit zu schreiben begleitet und unterstützt haben.

Als erstes möchte ich mich bei Herrn Prof. Dr. Konrad Samwer bedanken, der es mir ermöglicht hat, meine Promotion im 1.Physikalischen Institut anzufertigen. Ich habe in dieser Zeit sehr viel gelernt, da ich nicht nur von seinem großen physikalischen Weitblick profitiert habe, sondern vorallem hat er es mir ermöglicht auf vielen Tagungen und Seminaren das Gebiet der amorphen Materialien in seiner ganzen Breite kennenzulernen.

Bei Prof. Dr. Hans-Ulrich Krebs möchte ich mich dafür bedanken, dass er sich als Korreferent zur Verfügung gestellt hat.

Ein besonderer Dank gilt Prof. Dr. Ranko Richert, der mit seinem Aufenthalt in Göttingen erst den Impuls gegeben hat für die zeitabhängigen nichtlinearen Messungen. Dank seiner Unterstützung und den fruchtbaren Diskussionen ist das Projekt einen großen Schritt vorangekommen. Außerdem möchte ich mich für die Bereitstellung des Programmes zur periodennweisen Fouriertransformation bedanken.

Ganz herzlich möchte ich mich bei Katharina Tietz bedanken, die die Blockcopolymere hergestellt und am AFM charakterisiert hat. Die fachliche Zusammenarbeit am Thema hat mir sehr viel Spass gemacht und war dabei auch sehr effektiv.

Carsten Mahn möchte ich danken für den Umbau des DMA8000 für die nichtlinearen Messungen. Weiterer Dank gilt Kathrin Gehrke und dem gesamten Laborantenteam für die stets tatkräftige Unterstützung. Auch beim Werkstattteam und vor allem bei Herr Eichenberg möchte ich mich sehr bedanken, für die prompte und nette Erledigung aller Aufträge, selbst kurz vor Feierabend.

Besonderer Dank gilt meinen Bürokollegen Maik Schubert, Matthias BüchsenschützGöbeler und Birte Riechers für das angenehme Mitenander und die lustige und entspannte Atmosphäre. Vor allem, wenn die Experimente mal wieder nicht klappen wollten, hat mir der Austausch immer sehr geholfen und mich neu motiviert. Bei Birte möchte ich mich außerdem für die fundierten Diskussion über die Glasphysik bedanken und für die gemeinsamen verrückten Durchdreher, wenn wir nach stundenlangem Datenauswerten gesungen und geflucht haben.

Ganz herzlich möchte ich mich beim gesamten 1.Physikalischen Institut bedanken für das schöne Arbeitsklima. Besonders die gemeinsamen Seminarfahrten haben mir sehr gefallen. In der Zeit am Institut habe ich daher nicht nur viel gelernt, sondern 
auch sehr gute Freunde gefunden. In diesem Zuge möchte ich mich auch bei Melanie Schneider bedanken, die bei Problemen stets ein offenes Ohr hatte und mit der ich mich über die Herausforderung einer Dissertation mit Kleinkind austauschen konnte.

Mein größter Dank gilt meiner Familie, die mich stets unterstützt hat. Insbesondere danke ich meiner meiner Schwiegermutter Regina, die immer wieder Hagen liebevoll betreut hat, wenn ich länger gearbeitet habe oder eine Wochenendschicht eingelegt habe. Ein großer Dank gilt meiner Schwester Daniela für die Unterstützung bei allen kleinen und großen Krisen in den letzten Jahren und für die spontanen Einsätze bei der Betreuung von Hagen. Besonders danken möchte ich mich bei meinen Eltern, die mich stets gefördert haben und ein großes Vertrauen in meine Fähigkeiten hatten und dadurch erst ermöglicht haben, dass ich so weit gekommen bin. Meinem Mann Jan möchte ich ganz besonders herzlich danken. Die letzten Jahre hat er mir immer mit Liebe und Geduld zur Seite gestanden und mir in der anstrengenden Endphase den Rücken freigehalten. 